Chapman University

Chapman University Digital Commons

Education (PhD) Dissertations

Dissertations and Theses

Summer 8-2020

\title{
Secondary Traumatic Stress in Teachers and School Communities Impacted by the Opioid Epidemic
}

\author{
Anne Steketee \\ Chapman University, steke101@mail.chapman.edu
}

Follow this and additional works at: https://digitalcommons.chapman.edu/education_dissertations

Part of the Teacher Education and Professional Development Commons

\section{Recommended Citation}

Stekette, A. (2020). Secondary traumatic stress in teachers and school communities impacted by the opioid epidemic [Doctoral dissertation, Chapman University]. Chapman University Digital Commons. https://doi.org/10.36837/chapman.000176

This Dissertation is brought to you for free and open access by the Dissertations and Theses at Chapman University Digital Commons. It has been accepted for inclusion in Education (PhD) Dissertations by an authorized administrator of Chapman University Digital Commons. For more information, please contact laughtin@chapman.edu. 


\title{
Secondary Traumatic Stress in Teachers and School Communities Impacted by the Opioid Epidemic
}

\author{
A Dissertation by \\ Anne Steketee \\ Chapman University \\ Orange, CA \\ Attallah College of Educational Studies \\ Doctor of Philosophy in Education \\ August 2020 \\ Committee in charge: \\ Dr. Michael Hass, Chair \\ Dr. Randy Busse \\ Dr. Trisha Sugita \\ Dr. Nate Hosley
}

Submitted in partial fulfillment of the requirements for the degree of 
The dissertation of Anne Steketee is approved.

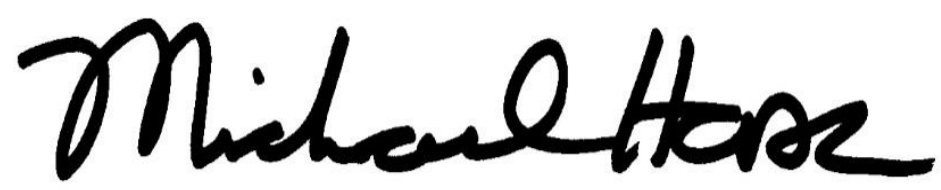

Michael Hass, Ph.D., Chair

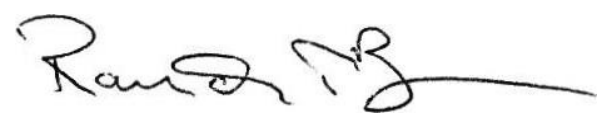

Randy Busse, Ph.D.

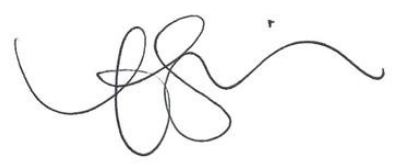

Trisha Sugita, Ph.D.

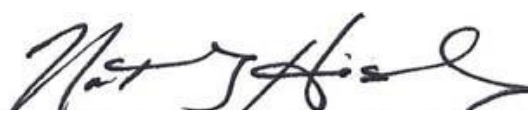

Nathaniel Hosley, Ph.D.

July 2020 
Secondary Traumatic Stress in Teachers and School Communities

\section{Impacted by the Opioid Epidemic}

Copyright $\odot 2020$

by Anne Steketee 


\section{ACKNOWLEDGEMENTS}

Every acknowledgement page tells a story. Often, it is the story of people who listened, friends who encouraged, family who waited, faculty who guided. My acknowledgements are no different.

This journey started with incredulity, grew into questions, then developed into a project that seemed to press in on my every weakness.

My lack was patiently tempered and strengthened by my committee, to whom I owe a debt of gratitude. Dr. Hass, Dr. Sugita, Dr. Busse, and Dr. Hosley--each added a unique and selfless contribution. Especially at the end, during the time of COVID-19, I valued your wisdom as we persevered together. And special thanks to Dawn Hunter and Sammi McCann. You are heroes to me, pulling me back from the brink again and again. And again.

The duration of the project, from inception to completion, was a family affair, riding on the incredible strengths of daughter, Cressie, and intrepid skills of husband, Casey. Said another way, the finished dissertation smacks of their love.

The questions and incredulity I owe to West Virginia Fire Chief Jan Rader. I heard her give a five-minute interview on TV. Those five minutes changed the direction of my dissertation by highlighting the crushing importance of the opioid epidemic and the humanity of those impacted by it.

And to Ashley, if this dissertation ever finds its way into your hands, you might be asking yourself these questions. I will go ahead and answer them for you, my sweet love.

Was I thinking of you as I wrote it?

I was. 
Are your children doing well?

They are.

Did you do the right thing for them?

You did.

Am I proud of you for that?

I am, every day.

Do I love you?

I do. 11 out of 10. Call me. Anytime 


\begin{abstract}
Secondary Traumatic Stress in Teachers and School Communities

Impacted by the Opioid Epidemic

by Anne Steketee
\end{abstract}

People who support others who have experienced trauma, like nurses, doctors, social workers, or first responders can sometimes be affected by a type of stress called secondary traumatic stress (STS). Although the effect of STS has been studied in helpers like social workers and medical professionals, the prevalence and characteristics of STS in teachers have not been studied extensively and are less understood. Schools in our communities impacted by the opioid epidemic also report additional stressors from issues like addiction, overdose, crime, neglect, rise in foster care, increased medical care, and death. This dissertation investigates STS in K-12 public school teachers in the United States, in areas of varying opioid impact. Specifically, K-12 teachers $(n=450)$, in 26 states and Washington, D. C., were surveyed utilizing a validated instrument for secondary traumatic stress (Secondary Traumatic Stress Scale; Bride, Robinson, Yegidis, \& Figley, 2004), along with demographic questions and open-ended questions. Teachers were also asked about adverse childhood experiences of their students, using the PHLACE categories (Health Federation of Philadelphia and Philadelphia ACE Research and Data Committee, 2012). The prevalence and extent of teacher STS were explored in communities of low-, medium-, and high-opioid impact levels as defined by the National Institute of Health epidemiology parameters. I used descriptive statistics and correlations (Spearman's Rho) to determine the prevalence of STS in the sample of teachers and to determine if this prevalence had any relationship to the opioid mortality rate in communities. Over half of the teachers in the 
study (59.56\%) experienced STS at a moderate or higher level. Teachers in high opioid zones reported the highest mean STSS scores $(M=43.78, \mathrm{SD}=16.00)$, with $62.67 \%$ scoring at 38 or higher. Over $85 \%$ of teachers endorsed intrusion symptoms at a diagnostic level. Between 91$93 \%$ of all teachers surveyed endorsed adverse events experienced by their students. Using Spearman's Rho correlation, I did not find a relationship between the environment of the opioid zone or the demographic characteristics of the teachers. Additional findings and implications are discussed and support the need to continue teacher STS research in all communities. 


\section{TABLE OF CONTENTS}

$\underline{\text { Page }}$

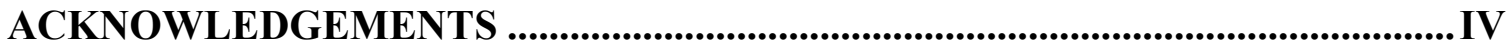

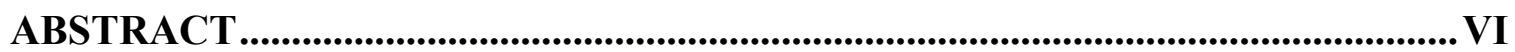

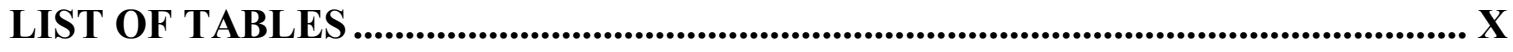

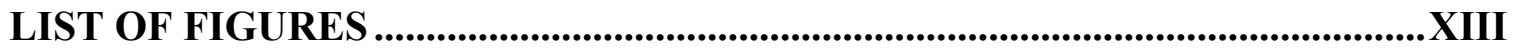

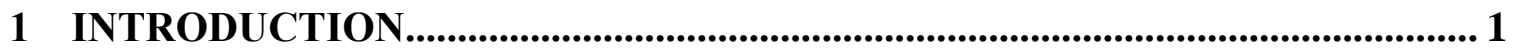

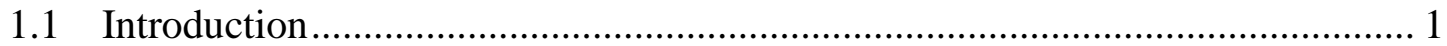

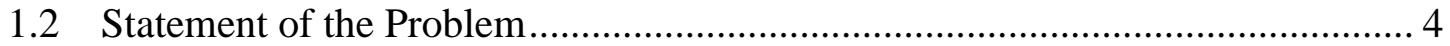

1.3 Purpose of the Study ................................................................................ 5

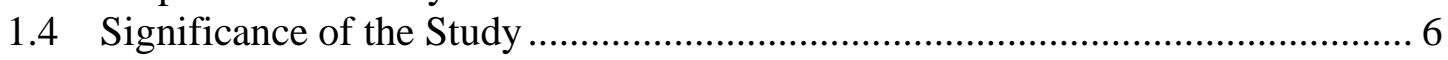

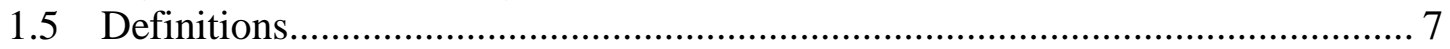

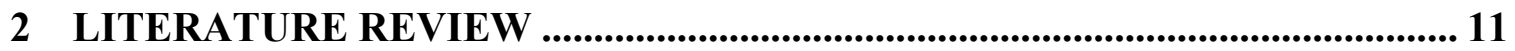

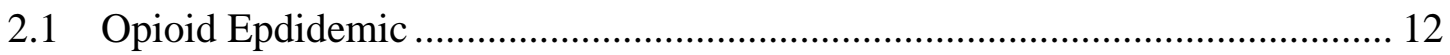

2.1.1 Socio-educational Impact of the Opioid Epidemic..................................... 13

2.1.2 Trauma Experienced by Children ..............................................................24

2.1.3 Secondary Traumatic Stress Framework .....................................................36

2.1.4 Secondary Traumatic Stress in Helping Professions..................................52

2.2 STS Risk Factors, Preventative Factors, and Interventions .............................. 61

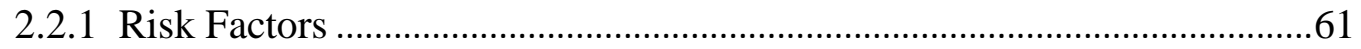

2.3 Secondary Traumatic Stress Experienced by Educators .................................. 65

2.3.1 Prevalence of Stress Experienced by Educators .......................................65

2.4 Secondary Traumatic Stress in High Opioid Epidemic Zones .......................... 77

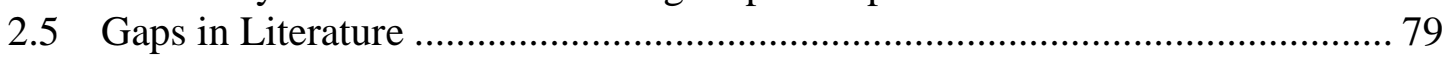

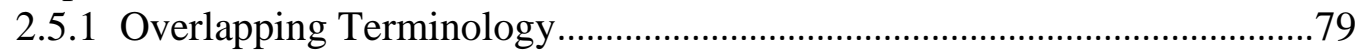

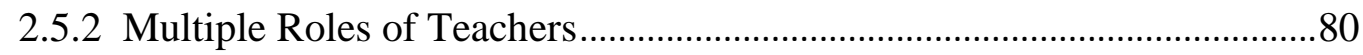

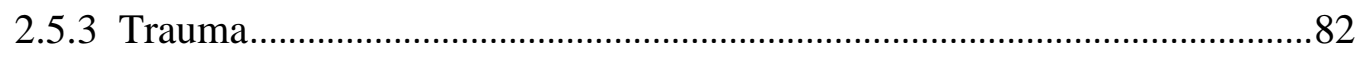

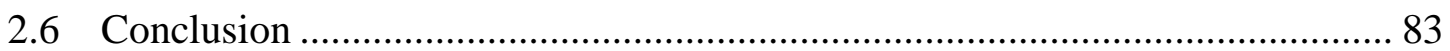

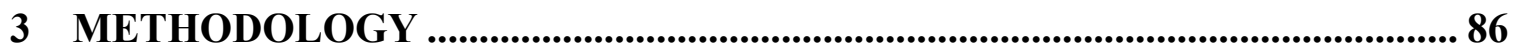

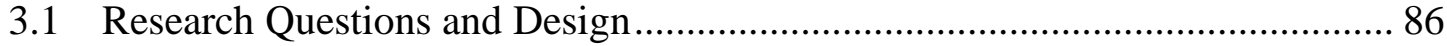

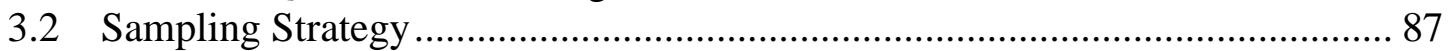

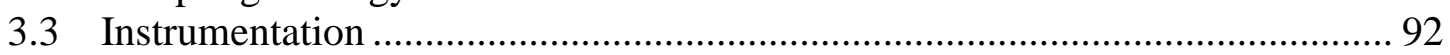

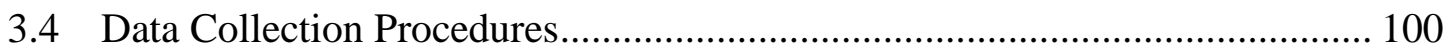

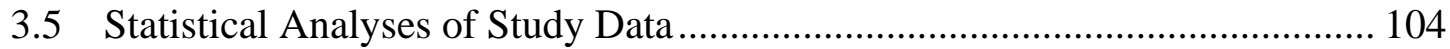

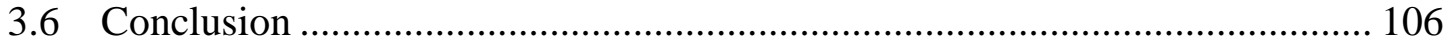




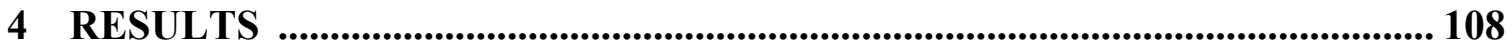

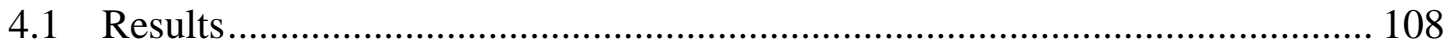

4.1.1 Measures and Plan of Analysis ............................................................. 108

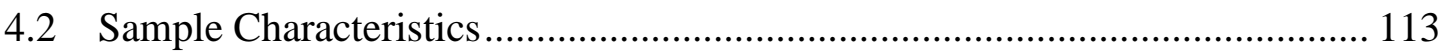

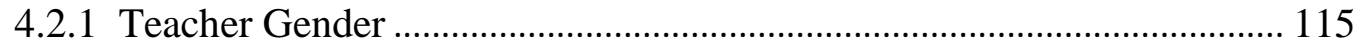

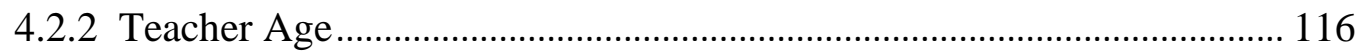

4.2.3 Teacher Relationship Status ................................................................. 116

4.2.4 Teacher Race/Ethnicity ...................................................................... 117

4.2.5 Teacher Primary Teaching Assignment ................................................... 118

4.2.6 Teacher Years of Experience.................................................................... 118

4.2.7 Teacher Report of Student Trauma (ACEs) ........................................... 121

4.3 Sample Secondary Trauma and Teachers ………………………………….... 123

4.3.1 Individual Question Results................................................................ 123

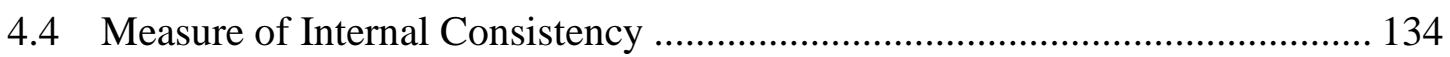

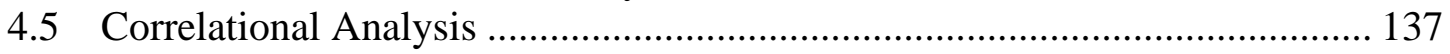

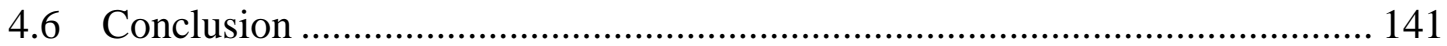

4.6.1 Research Question Summary ............................................................... 142

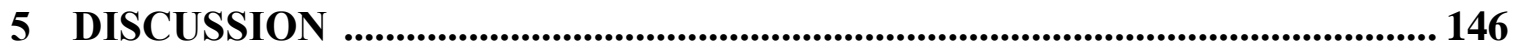

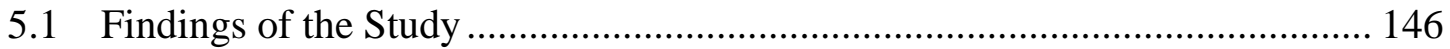

5.1.1 Prevalence of Teacher Reported STS..................................................... 146

5.1.2 Relationship of Teacher-Reported STS to Teacher Characteristics ....... 159

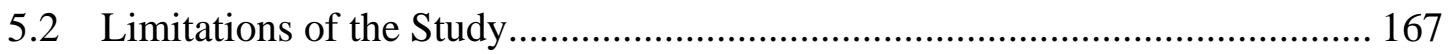

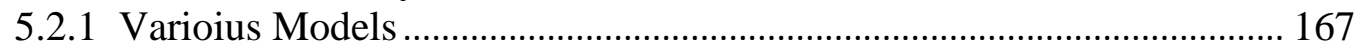

5.2.2 Teachers with "Disabilities" .................................................................... 167

5.2.3 Limited Number of Studies ................................................................. 169

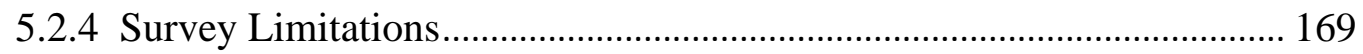

5.2.5 Disagreement about Diagnostic Features ................................................ 171

5.3 Implications, Strengths, and Future Research................................................ 173

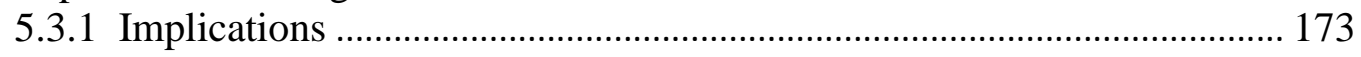

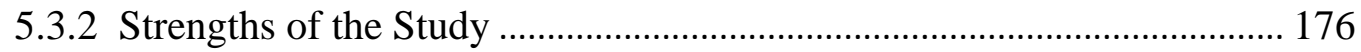

5.3.3 Recommendations for Future Research ……………………………..... 177

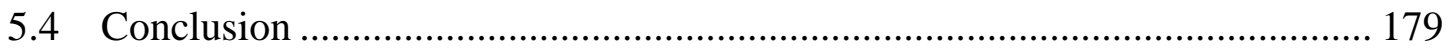

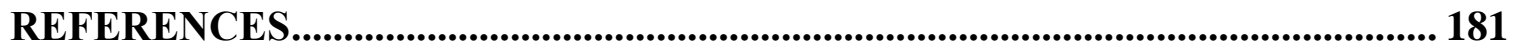

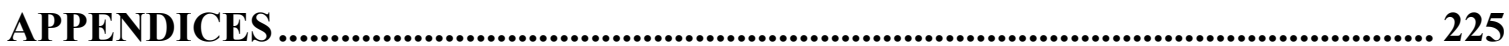




\section{LIST OF TABLES}

\section{Page}

Table 2-1: Comparison of Descriptive Opioid Epidemic Rates by State. ............ 16

Table 2-2: Variations in STS-related Definitional Comoponents........................ 37

Table 2-3: Comparison of DSM-IV-TR Diagnostic Criteria and STS Subscale Indicators.41

Table 2-4: DSM-V Inspired Traumatic Stress Models..................................... 50

Table 2-5: Comparison of Mean and Standard Deviation of Full STS Score. ..... 55

Table 2-6: Range and Percentile for the STS Subscales and Full Test................. 58

Table 2-7: Comparison of Mean and Standard Deviation Comparison for STS Subscales. 58

Table 2-8: Comparison of Frequency of STS Respondents Who Meet Criteria for One Subscale. 59

Table 2-9: Comparison of Frequency of Subscale Diagnostic Criteria of PTSD. 60

Table 2-10: Chronological Summary of Teacher STS Studies. 67

Table 3-1: Comparison of Mortality Sample Range. 89

Table 3-2: Criterion Validity of STSS within the Context of Additional Results.97

Table 4-1: Cronbach's Alpha Scale Interpretation.

Table 4-2: Demographic and Geographic Characteristics of K-12 Teachers..... 114

Table 4-3: Teacher Gender. ................................................................. 115

Table 4-4: Teacher Age Segments........................................................... 116

Table 4-5: Teacher Relationship/Marital Status. ........................................... 117

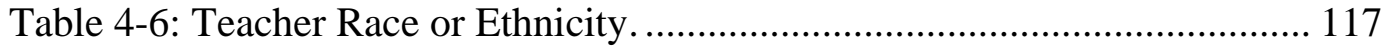

Table 4-7: Primary Teaching Assignment................................................... 118

Table 4-8: Years of Experience Teaching. .................................................... 119

Table 4-9: Frequency of Teacher Years of Experience. .................................. 119 
Table 4-10: Demographic Comparison National Teachers to Sample Teachers. 120

Table 4-11: Teacher Study Sample Range...................................................... 121

Table 4-12: Teacher National Age Range. ..................................................... 121

Table 4-13: Teachers' Perceptions of Student Adverse Childhood Experiences. 122

Table 4-14: Secondary Traumatic Stress Scale by Question for Total Sample $(\mathrm{N}=450)$. 124

Table 4-15: Secondary Traumatic Stress Scale by Question for Low Opioid Epidemic Zone $(\mathrm{n}=150)$. 125

Table 4-16: Secondary Traumatic Stress Scale by Question for Medium Opioid Epidemic Zone $(\mathrm{n}=150)$. 127

Table 4-17: Secondary Traumatic Stress Scale by Question for High Opioid Epidemic Zone $(\mathrm{n}=150)$ 128

Table 4-18: Results of the STSS Scale by Opioid Zone.................................. 129

Table 4-19: Results of the STSS Scale and Total Scores. ............................... 130

Table 4-20: Interpretation for the STSS Subscale. ......................................... 131

Table 4-21: STSS of Teachers Indicated by Using the Cutoff Method.............. 131

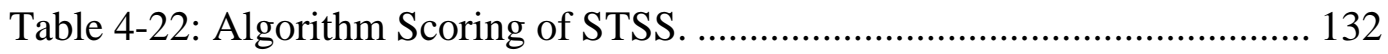

Table 4-23: Number and Percentage of Teachers at Each Level of the STSS (Summing/Percentile Method).

Table 4-24: Results of the Cronbach Alpha for Total Study Population by STSS Subscale. 135

Table 4-25: Results of the Cronbach Alpha by Opioid Zone for Total STSS.... 135

Table 4-26: Results of Cronbach Alpha for Subscales by Zone........................ 136

Table 4-27: Spearman's Rho by Opioid Zone $(\mathrm{N}=450)$............................... 139

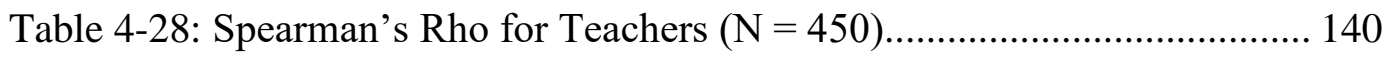

Table 4-29: Spearman's Rho Comparison of Low-Medium-High Correlations to Total Correlations.... 141

Table 5-1: Comparison of STS Study Mean for Full STSS Score. 148 
Table 5-2: CNumber and Percentage of Teachers at Each Level of the STSS (Summing/Percentile Method)...................................................................................... 149

Table 5-3: Results of the Cronbach Alpha for Total Study Population by STSS Subscale. 153

Table 5-4: Top Five Categories of Teachers' Perceptions of Study Adverse Childhood

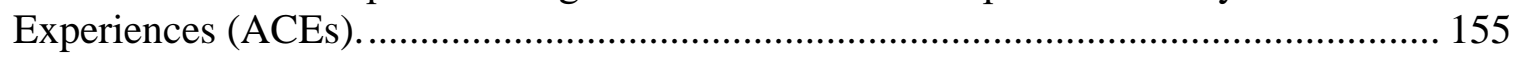

Table 5-5: Results of the STSS Intrusion Score. ............................................. 158

Table 5-6: Results of the STSS Avoidance Score. ............................................. 158

Table 5-7: Results of the STSS Arousal Score .................................................... 159

Table 5-8: Comparison of PHL-ACEs to Welby (2019) Trauma Categories..... 161

Table 5-9: Results of the STSS Scale by Opioid Zone........................................ 162 


\section{LIST OF FIGURES}

$\underline{\text { Page }}$

Figure 1-1 Process of Secondary Traumatic Stress in Teachers .........................6

Figure 2-1 Literature Review Aligned with the Process of Secondary Traumatic Stress in Teachers ............................................................................................... 12

Figure 2-2 School Trauma Factors and Process ..............................................26

Figure 2-3 Mechanism of Secondary Traumatic Stress in Opioid-Impacted

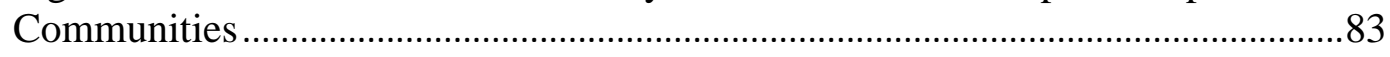

Figure 4-1 Akoglu's (2018) Spearman's Rho Correlations Coefficent Interpretation Comparison ......................................................................... 110

Figure 5-1 Frequency of Teachers with STS in Each Opioid Zone ................. 150

Figure 5-2 Percent of Teachers in STS Study .............................................. 152

Figure 5-3 PTSD Endorsement for Teachers in STS Study in Each Opioid

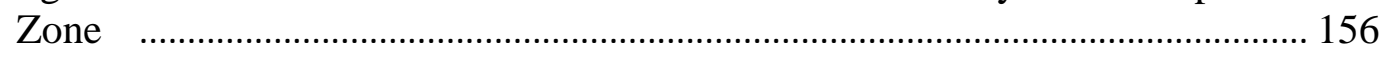

Figure 5-4 Teacher STS Subscale PTSD Percentage Compared to Other

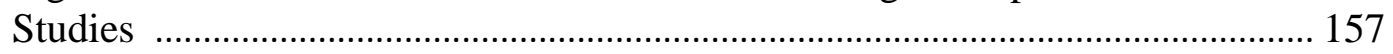

Figure 5-5 STSS Subscales of Teachers Using Algorithm Scoring ................ 159

Figure 5-6 Comparison of the Percentage of Teachers in STS Study Who Endorsed Intrusion Symptoms .......................................................................... 174 


\section{Introduction}

\subsection{Introduction}

People who support others in crisis, like nurses in an emergency room or social workers after a natural disaster, can sometimes be affected by secondary traumatic stress (Figley, 1999; Stamm, 1999). One definition of secondary traumatic stress (STS) is "the natural, consequent behaviors and emotions resulting from knowledge about a traumatizing event experienced by a significant other" (Stamm, 1999, p. 10). The effect of STS has been studied in helpers like social workers (Bride, Robinson, Yegidis \& Figley, 2004; Bride, 2007) and medical professionals (Granek, Nakash, Cohen, Ben-David, \& Ariad, 2017). The prevalence and characteristics of STS among teachers, however, have not been studied extensively and are less understood (Alisic, Bus, Dulack, Pennings, \& Splinter, 2012; Schepers, 2017; Wolf-Prusan, 2014).

Cieslak et al. (2013) describe professionals impacted by STS as dividing into two groups. First, there are those with either direct and indirect work-related trauma exposure, like rescue workers, firefighters, and doctors. Second are those with indirect work-related trauma exposure, like therapists, social workers, or childcare workers. Although there can be overlap between the two categories, as in the case with social workers (Cieslak et al., 2013), teachers would typically fall into this second category because they have indirect work-related trauma exposure due to their work with students in schools (Borntrager et al., 2012; Motta, 2012; Schepers, 2017). So while STS impacts professionals who are often categorized as first responders (Molnar et al., 2017; Stamm, 1999), it can also affect other types of professionals who work with people who have experienced trauma but who do not experience the trauma directly (Cieslak et al., 2013). Whether the trauma is experienced by working directly with people in acute trauma or indirectly 
by supporting people who are traumatized but in an acute crisis, STS manifests in a similar way (Hensel, Ruiz, Finney, \& Dewa, 2015; Stamm, 1999).

Because secondary traumatic stress (STS) results from supporting others who are impacted by trauma, understanding the sources of STS for teachers involves gaining a deeper understanding of the impact of trauma in student populations. The definition of trauma by the National Child Trauma Stress Network (NCTSN) is salient for school children and teachers, as child trauma can be any "experience that threatens life or physical integrity and that overwhelms an individual's capacity to cope. Generally, traumatic events evoke feelings of extreme fear and helplessness" (National Child Traumatic Stress Network, 2006, p. 9). The child's reactions are subjective, which means that they could differ depending on personal, developmental, social, and cultural factors. Therefore, what one child experiences as deeply traumatic, another child might experience as less traumatizing. Trauma, then, is the physiological and psychological reaction to an event of intensity or harm that threatens a child's physical and emotional well-being (National Child Traumatic Stress Network, 2006). Trauma can also result from chronic, prolonged, and repeated exposures that children experience over time (Van der Kolk, 2005). Felitti et al. (1998) described developmentally adverse events leading to trauma in children in the Adverse Childhood Experiences (ACE) study. These events - including abuse, neglect, family dysfunction, and family substance abuse-- can arise from an acute event or can develop over time as the result of chronic unrelenting stress.

The National Child Traumatic Stress Network (NCTSN; National Child Traumatic Stress Network, 2006) reported several applicable findings on child trauma. First, while trauma can be a response to an event like a natural disaster or war, it can also be the result of being the victim of violence or injury or even witnessing acts of violence or injury. Second, trauma reactions can 
occur at the moment or can persist over time. Children's responses to trauma tend to interfere with their ability to function in their daily lives. Additionally, children can react to traumatic events in different ways, depending on their development. For example, preschool children might have difficulty with separation anxiety, while elementary children might have a deterioration in school performance (National Child Traumatic Stress Network Schools Committee, 2008).

Nationally, $60.6 \%$ of children ages 0-17 years old, have experienced, witnessed, or been exposed to violence, abuse, or traumatizing events within the past year; $38.7 \%$ of children have been exposed to two or more incidents, with $10.9 \%$ reporting five or more occurrences of victimization during the year of the study (Finkelhor, Turner, Ormrod, \& Hamby, 2009). Although over $50 \%$ of children ages $0-17$ have been exposed to at least one traumatizing event, these students in their school placements may have behavioral or academic challenges, or they may not show any sign of their suffering (NCTSN, 2006, p. 14).

One of the adverse situations that can cause trauma in the lives of children is the use of opioids in the home (Stulac et al., 2019). The United States (U.S.) Department of Health and Human Services has identified opioid use disorder (OUD) as a national public health crisis (Macrae \& Hyde, 2015; Department of Justice, 2015) due to the high mortality rates (Hser et al., 2017) and the deleterious and chronic impact on families and communities (Stulac et al., 2019). Estimates place approximately eight million children living with at least one adult with substance use disorder (SUD), a disorder impacting many parents addicted to opioids (Dirks, 2018). Because these home environments can be highly unstable and chaotic, children may experience trauma or secondary trauma associated with opioids and SUD in the home (Lander, Howsare, \& Byrne, 2013; Stulac et al., 2019). Although some children are directly affected by opioids as a 
result of the effects of intrauterine opioid exposure on newborns and very young infants, trauma for most students impacted by the opioid health crises is due to opioid addiction impairing the functioning of the adults and caregivers. Since a large percentage of trauma-impacted children are either currently in school or will end up in school, Motta (2012) underscored the need for further research on STS in school settings for teachers who will be supporting students with trauma histories.

\subsection{Statement of the Problem}

Secondary traumatic stress is a construct that has been widely studied in professionals who support people who have experienced trauma (Ben-Porat, \& Itzhaky, 2011; Bride et al., 2004; Catherall, 1999; Chrestman, 1999; Granek et al., 2017; Hensel et al., 2015; KassamAdams, 1999; Markwell \& Wainer, 2009; McCann \& Pearlman, 1990; Morrison \& Joy, 2016; Pierce \& Lilly, 2012; Yager, Gerszberg, \& Dohrenwend, 2016). There is research to support the notion that teachers in high-stress communities may experience secondary stress; this includes communities with urban violence (Wolf-Prusan, 2014), communities impacted by poverty and the conditions that can sometimes accompany poverty (Denham, 2018), or communities profoundly affected by opioids (Anderson, Troilo, \& Tack, 2019; Welby, 2019). For educators who are teaching in environments of extreme stress, research focusing on STS could be significant professionally and personally. Professionally, teachers are positioned as "facilitators" for children with trauma, as change-agents for children's post-trauma recovery; however, teachers often feel they lack tools and training to be effective (Baum, Rotter, Reidler, \& Brom, 2009; Comer, 2005). Personally, teachers report feeling strain or burnout when working with children who have experienced trauma (Baum et al., 2009). Although classroom interventions by teachers can support students in their reactions to trauma, understanding the emotional burden 
for teachers when experiencing STS could also lead to better support for teachers (Alisic, 2012). Secondary traumatic stress (STS) has been studied in other professionals who support people who have experienced trauma, but teacher STS is less understood (Alisic, 2012; Schepers, 2017).

\subsection{Purpose of the Study}

The purpose of this study is to better understand the prevalence of STS in teachers and to determine if this prevalence has any relationship to the opioid mortality rate in communities. An additional purpose is to investigate the relationship between STS and teacher characteristics such as age, gender, race/ethnicity, age, and relationship status (married, single, etc.). This could help determine which variables, if any, are most related to higher levels of stress.

For these purposes, this study used survey data gathered from 450 teachers to answer three research questions predicated on three assumptions: (a) students are experiencing events leading to trauma, (b) students in certain communities are experiencing increasing and sustained trauma, and (c) teachers responding to these students daily may be experiencing STS.

- Research Question 1: What are K-12 teachers' self-reported levels of secondary traumatic stress?

- Research Question 2: Is there a relationship between teachers' self-reported levels of secondary traumatic stress when teachers are from states of high-opioid impact, states of medium-opioid impact, and states of low-opioid impact?

- Research Question 3: Is there a relationship between self-reported levels of secondary traumatic stress and the characteristics of teachers?

- Research Question 3a: Is there a relationship between self-reported levels of secondary traumatic stress and the age of teachers?

- Research Question 3b: Is there a relationship between self-reported levels of secondary traumatic stress and gender?

- Research Question 3c: Is there a relationship between self-reported levels of secondary traumatic stress and race/ethnicity? 
- Research Question 3d: Is there a relationship between self-reported levels of secondary traumatic stress and relationship status?

\subsection{Significance of the Study}

This research has the potential to help educational stakeholders understand more clearly the prevalence of teacher STS and the severity of teacher STS. The study might also elucidate the relationship between different teacher characteristics and secondary stress. The results could highlight strategies for targeted implementation of STS interventions for teachers in high-risk groups such as new teachers or teachers in high-stress communities. The risk of student trauma is increasing due to the reported rising opioid mortality rate in our communities (Edelman, 2017; Stulac et al., 2019). This increased student risk means that teachers who respond to student trauma are potentially at prolonged and continuous risk for developing secondary traumatic stress (Dirks, 2018; Motta, 2015; Schepers, 2017). In Figure 1-1, the process of STS is demonstrated, showing the impact on both the student and the teacher. An overarching goal of this study is to understand secondary trauma in our school communities and use this understanding to better support both student and teacher.

Figure 1-1: Process of Secondary Traumatic Stress in Teachers

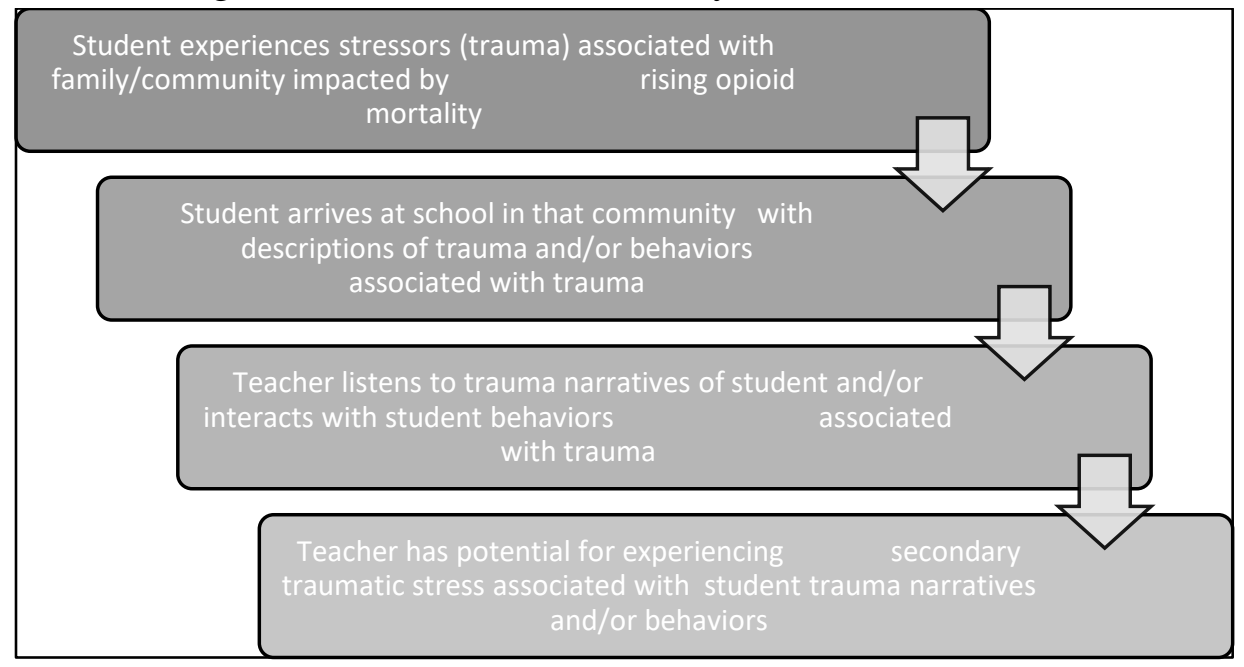

Note. Figure showing antecedent and progression of secondary traumatic stress for a teacher who supports a student with trauma history. 


\subsection{Definitions}

Some definitions ground the understanding of the discussion of secondary traumatic stress. The central definitions are the following:

Adverse Childhood Experiences (ACEs): Adverse events leading to trauma in children. Described in the Adverse Childhood Experiences (ACE) study to include events like abuse, neglect, family dysfunction, and family substance abuse, which can arise from an acute event or can develop over time as the result of chronic unrelenting stress (Felitti et al., 1998).

Arousal: One of three subscales on the Secondary Traumatic Stress Scale (STSS; Bride et al., 2004), based on arousal symptoms of post-traumatic stress disorder (PTSD; American Psychiatric Association, 2000). Arousal symptoms include jumpiness, difficulty sleeping, easily startled, difficulty concentrating, irritability, and hypervigilance. On the STSS, the arousal subscale is located on questions $4,8,11,15$, and 16 .

Avoidance: One of three subscales on the Secondary Traumatic Stress Scale (STSS; Bride et al., 2004), based on avoidance symptoms of post-traumatic stress disorder (PTSD; American Psychiatric Association, 2000). Avoidance symptoms include emotional numbing; foreshortened future; detachment from others; diminished activity level; avoidance of people, places, and things; avoidance of students; and inability to recall student information. On the STSS, the avoidance subscale is located on questions 1, 5, 7, 9, 12, 14, and 17.

Burnout: Different from stress in that it is a byproduct of stress that is prolonged, burnout is multidimensional: it can produce exhaustion that is emotional, physical, or attitudinal (Travers, 2017).

Compassion fatigue: A condition that can result from the accumulated effect of caring for others, resulting in adverse physical, emotional, or cognitive impact (Figley, 1995). 
Diagnostic and Statistical Manual of Mental Disorders (DSM-IV): The manual developed by the American Psychiatric Association to provide a standardized classification system for the diagnosis of child and adult mental health disorders. The fourth edition was used for the development of the Secondary Traumatic Stress Scale (STSS; Bride et al., 2004).

Epidemic: In public health terminology, epidemic has a particular meaning that includes spread from an infectious agent (Centers for Disease Control, 2012). In this study, I use epidemic as it is used in the literature to refer to conditions that have origins that are behavioral, genetic, or psychosocial, similar to Alzheimer's disease and obesity (Brundage \& Levine, 2019). A complete explanation is found in chapter two of this study.

Hotspot: Spatial clusters with "elevated incidence or prevalence, higher transmission efficiency or risk, or higher probability of disease emergence" (Lessler, Azman, McKay, \& Moore, 2017, p. 1270). Lessler et al. (2017) recommended explicitly defining the hotspot with modifiers, for clarity. For this study, opioid hotspots are identified by the intersection of mortality rate and annual percent of change for mortality rate (Kiang, Basu, Chen, \& Alexander 2019). In this way, the modifier would be mortality hotspot.

Intrusion: One of three subscales on the Secondary Traumatic Stress Scale (STSS; Bride et al., 2004), based on intrusion symptoms of post-traumatic stress disorder (PTSD; American Psychiatric Association, 2000). Intrusion symptoms include physiological reactivity, reliving students' trauma, psychological distress, intrusive thoughts, and disturbing dreams. On the STSS, the intrusion subscale is located on questions $2,3,6,10$, and 13.

Opioid: Opioids are a broad category of drugs with chemical structures that work at one or more of the body's opioid receptors. These can include some opiates (substances extracted from opium), along with their derivatives (like heroin, oxycodone, hydrocodone, 
hydromorphone, and buprenorphine), compounds that are entirely synthetic (such as fentanils, meperidine, methadone), and natural peptides produced by the body known as endogenous opioids, such as endorphins (Newton, 2018).

Opioid epidemic: Price (2017) announced the U.S. national health crisis as "the opioid epidemic" on April 19, 2017. He referred to the "addiction crisis that is ravaging our country" and mentions the drugs heroin, fentanyl and carfentanil, prescription drugs, and opioid addiction.

Opioid epidemic zone: An opioid epidemic zone is a geographic area, informed by the understanding of hotzone, as detailed by Kiang et al. (2019). When identified by the intersection of mortality rate and annual percent of change for mortality rate, a determination was made to define a low opioid epidemic zone, a medium opioid epidemic zone, and a high opioid epidemic zone.

Opioid Use Disorder (OUD): Brundage and Levine (2019) defined OUD as "problematic pattern of opioid use leading to clinically significant impairment or distress" (p. 37).

Posttraumatic Stress Disorder (PTSD): After exposure to a traumatic event, posttraumatic stress disorder (PTSD) can develop, leaving a person feeling like there stress or threat, even when the threat has passed (National Institute of Mental Health, 2019). PTSD has four groups of symptoms, which include the following: (1) memories of trauma that are intrusive and recurrent, (2) avoidance of trauma-related stimuli, (3) changes in mood that are negative or numbing or changes in thoughts about the trauma, and (4) reaction and arousal changes (American Psychiatric Association, 2013).

Secondary traumatic stress (STS): Secondary traumatic stress (STS) results from helping or wanting to help a traumatized person. Stamm (1999) defined STS as "the natural, consequent behaviors and emotions resulting from knowledge about a traumatizing event experienced by a 
significant other" (p. 10). The identification of STS can be made when assessed by the Secondary Traumatic Stress Scale (STSS; Bride et al., 2004).

Substance Use Disorder (SUD): Brundage and Levine defined substance use disorder (SUD) as the following: "individuals with OUD may also use other nonopioid substances, and that many of the challenges facing families affected by the current opioid epidemic — and the proposed recommendations_-apply more generally to SUD” (p. 37). In this way, SUD is a broader term than OUD. Some researchers use them interchangeably.

Trauma: Multiply defined construct that is often referred to as an emotional response to a stressor. The multiple definitions tend to focus on the severity of the stressor, with some theorists holding to a more precise description (Saunders \& Adams, 2014) and others advocating more breadth (Cole, Eisner, Gregory, \& Ristuccia, 2013). 


\section{Chapter Two: Literature Review}

Although the effect of STS has been studied in helpers who deal with trauma, the topic of STS with teachers has not been considered as extensively and is, therefore, less understood (Alisic et al., 2012; Schepers, 2017; Wolf-Prusan, 2014). As the opioid epidemic increases (Jones, Logan, Gladden, \& Bohm, 2015), issues like addiction, overdose, crime, neglect, foster care, medical need, and death also increase (Anderson et al., 2019; Hefling \& Stratford, 2018; Radel, Baldwin, Crouse, Ghertner, \& Waters, 2018). A rise in parent opioid misuse and overdose death have led to increases in adverse childhood experiences (ACEs) and foster care placements for children (Feder, Letourneau, \& Brook, 2019; Patrick \& Schiff, 2017; Radel et al., 2018). Educators are tasked with working as secondary responders to these trauma-inducing events in the prolonged crisis (Landers, 2018). Teachers in states that have been impacted with high opioid mortality and subsequent increases in traumatizing issues for children have been tasked with additional responsibilities. These responsibilities include stocking opioid antidotes at school (Opioid Prevention Act, 2018), instructing students regarding opioid abuse and administration of antidotes (Gray, Capote \& Valiente, 2016), dealing with neglect (Feder, Letourneau, \& Brook, 2019; Welby, 2019), handling increased challenging student behaviors (Welby, 2019), and coping with increasingly high absenteeism (Engberg \& Morral, 2006; Feder et al., 2019). An additional theme found in the research literature is that teachers are beginning to note difficulty with some parents, including secretiveness, guardedness, and neglectful attitudes (Welby, 2019). Further information regarding the impact of these challenges on teachers and, more specifically, their experience of secondary stress is needed. 


\subsection{Opioid Epidemic}

The first section of this chapter reviews the literature regarding the socio-educational impact of the opioid epidemic in communities and schools. This research provides a context for the subsequent discussion about trauma experienced by children in communities affected by stress. This discussion of the impact of the opioid epidemic and its effects on communities and children highlights the salience of the final section on secondary traumatic stress (STS). The STS literature, vital to the study, coalesces around three themes: STS framework, STS with educators, and STS in high opioid epidemic zones. Finally, gaps in the literature will be discussed. Figure 2-1 (below) shows how the literature review seeks to elucidate the process of STS, with an impact on both student and teacher.

Figure 2-1: Literature Review Aligned with the Process of Secondary Traumatic Stress in Teachers

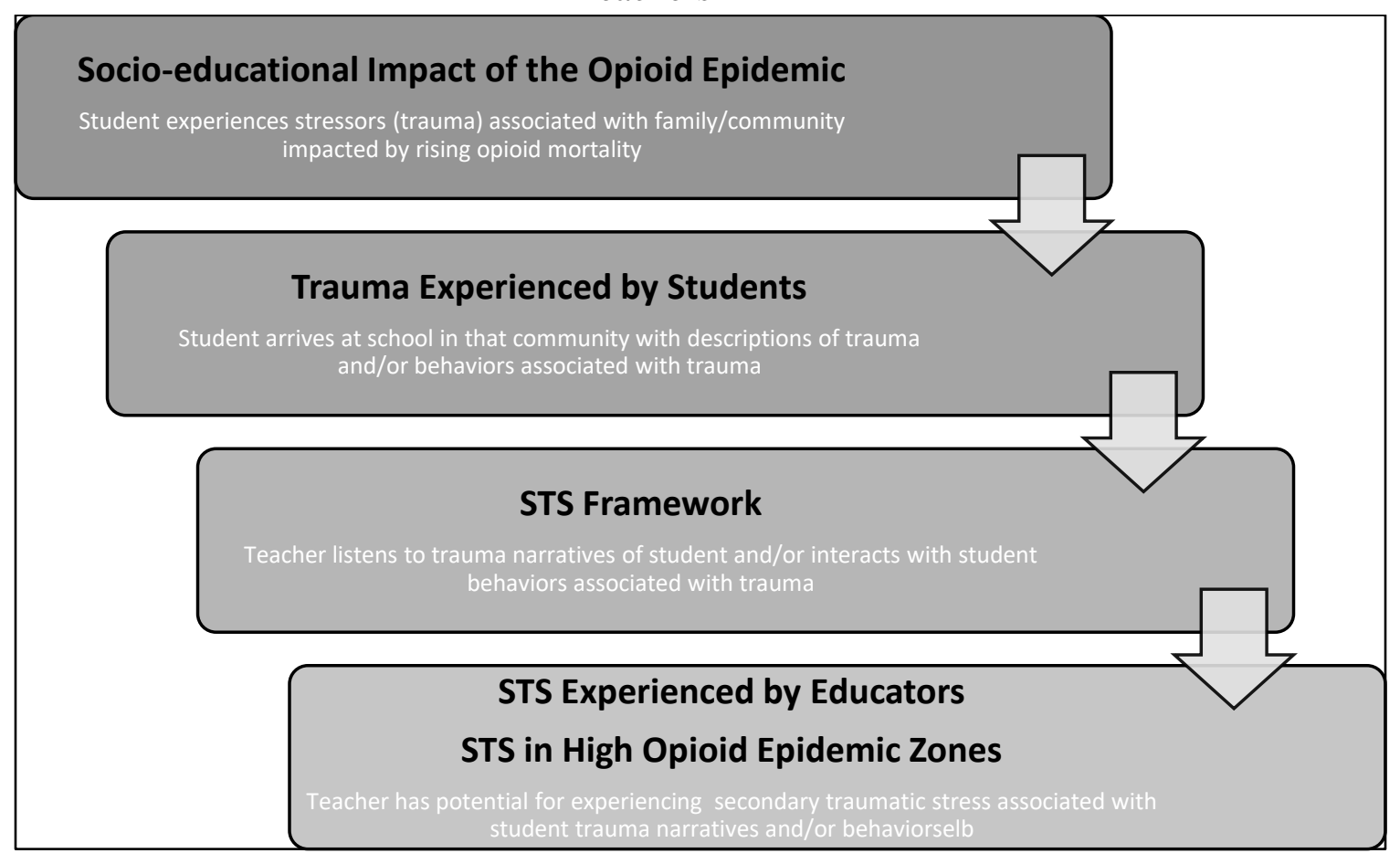

Note. Figure 2-1 builds on the process from Figure 1-1, aligning the literature review topics for the reader. The literature review is listed in black font, while the process continues to be described in white font. 


\subsubsection{Socio-educational Impact of the Opioid Epidemic}

The president's commission on combating drug addiction deems the nation at a crisis because of the number of deaths due to opioid drug overdose. Deaths related to opioid use now occur at a rate higher than mortalities from gun homicides and car fatalities combined (Christie et al., 2017). Madras (2018) located over 30 factors causing the crisis. These include deficiencies in the U.S. health care system, the rising need to manage chronic pain, "scientifically questionable" research supporting the overuse of opioids, and aggressive campaigns on the part of the pharmaceutical industry promoting the use of opioids to deal with pain (p. 943). The following section of this chapter will examine four aspects of the opioid epidemic: (a) brief background information; (b) definition in the research literature; (c) exploration of the impact on communities, families, and children; and (d) analysis of the effect on schools and educators. This literature review is not intended as a commentary on any medically-indicated treatment, between doctors and patients, involving opioids. The sole goal is to provide a socio-educational understanding of the impact of the opioid epidemic on children and schools to inform the research centering on teachers and secondary stress in our communities.

Brief background information. Opium, in pure form, is a crystal-like white powder; it begins as a fluid-like substance that is extracted from the seeds of the poppy plant (Papaver somniferum), which is then dried to create crystalline opium (Newton, 2018). The poppy plant yields opiate. Opioids act on receptors in the brain to reduce pain, induce sleep, or produce a pleasurable sensation (Olsen \& Sharfstein, 2019). At times, there is confusion between opiates and opioids. Reisfield, Bertholf, and Wilson (2007) described opiates as "substances extracted from the milky latex of ripening pods of the opium poppy" (p. 179). They further divide them 
into two classes: (1) phenanthrenes, which include morphine, codeine, and thebaine; and (2) benzylisoquinolines, which include papaverine and noscapine. Unlike opiates, opioids are a much broader category of drugs with a broad range of chemical structures that work at one or more of the body's opioid receptors. Although these can include some opiates, along with their derivatives (like heroin, oxycodone, hydrocodone, hydromorphone, and buprenorphine), opioid compounds also include those that are entirely synthetic (such as fentanils, meperidine, methadone), along with natural peptides produced by the body known as endogenous opioids such as endorphins (Newton, 2018).

Opioid use and misuse, along with the derivatives of opioids, have a history that dates back to early civilizations. Rudgley (2000) places the earliest date at around 6000 BCE, with other historians placing opium's entrance to the time of the Sumerians in 2100 BCE (Newton, 2018). In the $16^{\text {th }}-19^{\text {th }}$ centuries, Newton (2018) noted expanding attitudes and developing practices toward opium as a medical product in Europe, China, India, and even the United States. In 1942, the U.S. Congress passed the Opium Control Act, which prohibited the growing of poppies, and in 1956 the U.S. passed the Narcotic Control Act, which increased the penalties for importing, selling, or using opiates (Newton, 2018). In the United States, the Comprehensive Drug Abuse Prevention and Control Act was adopted in 1970. This act both consolidated all laws related to drugs and established a classification system for drugs. Opium and its derivatives became Schedule I drugs. In 1986, the Anti-Drug Abuse Act classified all opiates, opioids, and derivatives as Schedule I drugs. The Drug Addiction Treatment Act of 2000 gave physicians the ability to treat patients with opioid addictions in their offices and to provide patients medications for these treatments. A 300\% increase between 1991-2010 is noted for the number of prescriptions for opioids. In 2017, President Donald Trump declared a national health 
emergency. Finally, between 2002-2017, there was a $280 \%$ increase in the number of Americans who died of opioid overdoses. Throughout the timeline, Newton (2018) noted that both the medicinal and recreational uses of opioids have increased.

Definition of the opioid epidemic in the research literature. The opioid epidemic has been determined methodologically by researchers in different ways. One way is by tracking mortality (Kiang et al., 2019), another approach is by noting hospitalizations (Weiss et al., 2017), and a third way is by assessing the financial cost (Florence, Zhou, Luo, \& Xu, 2016). For this study, the definition of the epidemic has been aligned with public health principles, epidemiology definitions, and mortality rates.

Epidemic. In public health and epidemiology, an epidemic has a prescribed definition for infectious agents that "refers to an increase, often sudden, in the number of cases of a disease above what is normally expected in that population in that area" (Centers for Disease Control and Prevention, 2012a). But the term "epidemic" can also be used for non-infectious issues like obesity or diabetes if they occur in epidemic proportions (Centers for Disease Control and Prevention, 2004; Mitchell, Catenacci, Wyatt, \& Hill, 2011; Zimmet, 2017). Because of the percentage of deaths, the opioid crisis has been deemed an epidemic (Kiang et al., 2019).

Another aspect that defines an epidemic is the magnitude of its impact. For the opioid epidemic, Brundage and Levine (2019) noted the following statistics: from 2009-2014, eight million children lived with at least one parent with SUD; from 2004-2013, the incidence of NAS tripled in the United States; and since 2012, there has been a 10\% increase in foster care placements, partially due to the opioid epidemic (Ghertner, Baldwin, Crouse, Radel, \& Waters, 2018). In 2015, $23 \%$ of all opioid deaths were among adults aged $34-44$, and $26 \%$ of all opioid deaths were among adults aged 25-34. Ages 25-44 are the prime parenting years (Brundage \& 
Levine, 2019). Finally, Normile, Hanlon, and Eichner (2018) reported that the estimated cost of the opioid epidemic, between 2001 and 2017, exceeded one trillion dollars. This amount is derived from lost productivity and increased spending on vital areas like health care, social services, education, and criminal justice.

Although opioid abuse is found in all 50 states (Centers for Disease Control and Prevention, 2020b), there is both a variation in the rate of mortality due to overdose and a variation in other statistical indicators of epidemic description between the states. Table 2-1 describes the mortality rate (per 100,000) and the rate of children affected by the opioid epidemic (per 1,000) for the states represented in this study. California, which for this study has been identified as a low opioid zone state, has a death rate at $12.8 \%$ and a child impact rate at $20 \%$. North Carolina, a medium opioid zone state, has a death rate at $22.4 \%$ and a child impact rate at 30. Delaware, in the high opioid zone state classification, has a death rate at $43.8 \%$ and a child impact rate at $41 \%$. This variation within the states is noticeable in the institutions of the state school systems. For example, the California Department of Education (https://www.cde.ca.gov/), accessed on June 19, 2020, had three results when "opioid" was used as a search term. The same search, the same day, on the Ohio Department of Education website (http://education.ohio.gov/) produced 75 results. Different states, because of the different rates of OUD, have focused on different educational issues.

Table 2-1: Comparison of Descriptive Opioid Epidemic Rates by State

\begin{tabular}{|c|c|c|}
\hline State & $\begin{array}{c}\text { Death Rate (per } \\
\mathbf{1 0 0 , 0 0 0 )} \mathbf{2 0 1 8}^{\mathbf{a}}\end{array}$ & $\begin{array}{c}\text { Rate of children } \\
\text { affected by the } \\
\text { opioid epidemic } \\
\text { in 2017 }\end{array}$ \\
\hline Alabama & 16.6 & 37 \\
\hline Arizona & 23.8 & 31 \\
\hline California & 12.8 & 20 \\
\hline
\end{tabular}




\begin{tabular}{|c|c|c|}
\hline Colorado & 16.8 & 29 \\
\hline Connecticut & 30.7 & 39 \\
\hline Delaware & 43.8 & 41 \\
\hline Florida & 22.8 & 31 \\
\hline Georgia & 13.2 & 23 \\
\hline Louisiana & 25.4 & 24 \\
\hline Maine & 27.9 & 38 \\
\hline Maryland & 37.2 & 32 \\
\hline Massachusetts & 32.8 & 31 \\
\hline Minnesota & 11.5 & 24 \\
\hline Mississippi & 10.8 & 34 \\
\hline Missouri & 27.5 & 32 \\
\hline Montana & 12.2 & 31 \\
\hline New Hampshire & 35.8 & 51 \\
\hline New Jersey & 33.1 & 32 \\
\hline North Carolina & 22.4 & 30 \\
\hline Ohio & 35.9 & 32 \\
\hline Pennsylvania & 36.1 & 33 \\
\hline South Carolina & 22.6 & 29 \\
\hline South Dakota & 6.9 & 25 \\
\hline Tennessee & 27.5 & 31 \\
\hline Texas & 10.4 & 23 \\
\hline Wisconsin & 19.2 & 25 \\
\hline
\end{tabular}

${ }^{a}$ The number of deaths per 100,000 total population (Source: Centers for Disease Control and Prevention, 2020b). ${ }^{\mathrm{b}}$ Source: Brundage, Fifield, and Partridge (2019).

Brundage, Fifield, and Partridge (2019) noted that opioids impact 2.2 million children. The national median for children impacted by the opioid epidemic is 28 out of 1,000 (Brundage et al., 2019). While the rates for children in California, Texas, Florida, and New York (28\%) are at or below this national average, the actual numbers of children impacted in these states make up almost $30 \%$ of the total number of children impacted due to the high population in these states: California $(196,000)$; Texas $(171,000)$; Florida $(138,000)$; and New York $(125,000)$. So 
while these states may not be high for child impact percentage, together they represent almost one-third of the nation's children.

Impact of the opioid epidemic on families and children. Feder (2018) identified four pathways that adult opioid use impacts children: 1) maternal opioid use during pregnancy, 2) maladaptive family interaction and attachment, 3) deprivation and neglect, and 4) extended separation from parents.

Maternal opioid use. Because opioids cross the placenta, a developing fetus will receive some opioids from a pregnant mother who is using opioids (Olsen \& Sharfstein, 2019). Olsen and Sharfstein (2019) noted that this use could cause fetal distress, higher risk for additional infections (like HIV or HepC, etc.), fetal heart infections, or even death. If the pregnant mother overdoses, the impact on the developing fetus can be catastrophic. Neonatal abstinence syndrome (NAS) describes a cluster of problems that occur in newborns who were exposed in utero to opioids or opiates for a duration ("Neonatal abstinence syndrome," 2019). The symptoms of NAS are dependent on the type of drug used, the duration, the amount, genetic factors of maternal metabolism, and the developmental cycle of the fetus at the time of maternal drug use ("Neonatal abstinence syndrome," 2019). Hudak, Tan, Committee on Drugs, and Committee on Fetus and Newborn (2012, p. 545) noted that the neurological features of NAS withdrawal for the infant could include such symptoms as tremors, irritability, increased wakefulness, highpitched crying, and seizures, while the gastrointestinal features can include symptoms like poor feeding, uncoordinated and constant sucking, vomiting, diarrhea, dehydration, fever, and poor weight gain.

Maladaptive family interaction and attachment. Mirick and Steenrod (2016), through literature review, noted that OUDs are negatively associated with child welfare involvement, 
with longer foster care stay for children when compared to alcohol, methamphetamine, or marijuana use and slower reunification with parents. Additional reported findings include a more limited range of parental responsiveness toward children and insecure attachment. Numerous studies report such findings. I am cautious about reflecting on many of them at this time for two reasons: (1) the focus of this dissertation is teacher response to students in classrooms, not an exhaustive critique of social and familial issues, and (2) the variables involved in attachment research like this are, and continue to be, complex (Schindler, 2019). Limitations in this research include the following: a) attachment is not a single variable, making it difficult to differentiate between types of attachment; b) different substances (heroin, cocaine, etc.) and severities yield different findings; c) support for the notion that people with SUD/OUD may have an underlying attachment issue which predates and possibly enhances the development of SUD (Schindler, 2019). The field of neurobiology (Strathearn et al., 2019), which describes interactions between the molecular, neuroendocrine, and behavioral levels of experience, might broaden discussions of attachment.

Deprivation and neglect. With collated data from 2014-2016, the U.S. Department of Health and Human Services reported that an estimated 28.5\% of substantiated cases of child maltreatment and neglect are associated with caregivers who abuse drugs (U.S. Department of Health \& Human Services et al., 2018). This rate is significantly higher than the risks associated with an alcohol abuse caregiver at 11.5\% nationally for the same time. Smith and Wilson (2016) noted that parents with SUD might have difficulty providing the basic needs for children, including regular dental and medical checkups with medical professions who are uniquely positioned to intervene in issues of neglect. 
Altshuler and Cleverly-Thomas (2011) found that parents who use substances and abuse alcohol have children who are four times as likely to be physically deprived or emotionally neglected. Smith and Wilson (2016) described common signs of neglect: lack of personal hygiene, ill-fitting or inappropriately weather-safe clothing, poor school attendance, lack of supervision, and lack of nutrition and shelter. It should be noted that this impairment in parenting is variable and depends on the geographic area of the family (Chasnoff, Telford, Wells, \& King, 2015) and the type of opioid or opiate (Slesnick, Feng, Brakenhoff, \& Brigham, 2014). Financial demands of SUD are noted as a risk factor for neglect of children (Callaghan, Crimmins, \& Schweitzer, 2011; Smith \& Wilson, 2016).

Extended separation from parents. Radel et al. (2018), from the U.S. Department of Health and Human Services, detailed the positive association between parental drug use and child welfare cases and placements. As the rates of drug-related hospitalization and overdose deaths increase, there is a statistical relationship with rates of child protective services reports, substantiated reports, and foster care placements. In general terms, communities with higher drug rates have higher child welfare rates. Additionally, the SUD indicators also are related to rates of more complicated and severe social welfare cases for children. Before 2012, foster care rates had been decreasing nationally; however, between 2012 - 2016, the number of children placed in foster care rose $10 \%$ (from 397,600 to 437,500), with six states noting a 50\% increase over the four years (Radel, Baldwin, Crouse, Ghertner, \& Waters, 2018b). Radel et al. (2018b) utilized county-level prevalence of two indicators of substance use and three measures of child welfare caseloads, negative binomial regression models, and the false discovery rate (FDR), as defined by Benjamini and Hochberg (1995), where 0.05 was set as the FDR threshold. For a detailed account of the full model results, see Ghertner, Waters, Radel, and Crouse (2018). 
Effect of the opioid epidemic on schools and educators. Newton (2018) reported that opioid abuse prevention experts recommended that educational programs should be made available for students at every level, from prekindergarten to college. While these programs come from a variety of sources - commercial and professional publishers, government agencies, state departments of education, and even school districts and individual schools — the people teaching these programs are often educators within the educational settings, most often teachers (Newton, 2018).

An example of this type of curriculum, mandated for educators to include in their core of teaching standards, comes from the Ohio Department of Education (Ohio Department of Education, 2020):

In grades kindergarten through third, instruction should include 'differences among foods, poisons, medicines, and drugs; personal responsibility for one's actions;' and rules regarding who provides, distributes, accesses and monitors medication in the home and community. (para. 6)

The instructions became more detailed for higher grades, including "emphasis on the potential progression of addiction that could lead to heroin addiction and potentially death" (para. 9) for grades six through eight and "how students can help a friend or family member who may be addicted to prescription pain medicines and/or heroin" (para. 11) for grades nine through twelve. These are important goals, but also could add considerably to the teaching load of educators in K-12 settings - not just in terms of cognitive content, but also in terms of emotional content.

This educational mandate is not a suggestion for best practice from the Ohio Department of Education. 33 Ohio Rev. Code (2014) states that the health education curriculum for each school district "shall include instruction in [...] prescription opioid abuse prevention." Teachers 
in Ohio and other states are required to add additional drug prevention content to core curricula, which can benefit students (Welby, 2019) but can burden teachers (Welsh, Tretyak, \& Rappaport, 2018). Welsh et al. (2017) noted that teachers are impacted in a variety of ways: because of stigma surrounding opioids, teachers may experience barriers to provide support; teachers also commonly report feeling unprepared to recognize drug use and to deal with overdose; and teachers and administrators note an uncertainty regarding services for students with disabilities, due to overlap with substance abuse issues.

In addition to curricula and program creation for schools and educators, there is a continual need for child development applications informed by research about opioids. There is a positive relationship between children affected by opioids in vitro by neonatal abstinence syndrome (NAS) and the need for specialized educational services (Beckwith \& Burke, 2015; Fill et al., 2018). Fill et al. (2018) analyzed health records $(\mathrm{N}=7,256)$ of children with NAS $(\mathrm{n}=$ 1815) and children without NAS $(n=5441)$ They found the following: children with NAS were more likely to be referred for special education or disability evaluation (351 of 1815 [19.3\%] vs. 745 of $5441[13.7 \%] ; P<.0001)$; children with NAS were more likely then to meet the criteria for disability (284 of 1815 [15.6\%] vs. 634 of 5441 [11.7\%]; $P<.0001$ ); and finally children with NAS were then more likely to be eligible for special education services, therapies, or classroom interventions (278 of 1815 [15.3\%] vs. 620 of 5441 [11.4\%]; $P<.0001)$. The researchers controlled for maternal variables. NAS has a far-reaching impact on students' educational trajectories.

Although the United States has a marked increase in NAS birthrate, increasing nationally from 3.4 (95\% confidence interval (CI): 3.2 to 3.6) to 5.8 (95\% CI 5.5 to 6.1$)$ per 1000 hospital births (Patrick, Davis, Lehmann, \& Cooper, 2015), NAS is a global issue (Davies et al., 2015). 
The educational implications are also global. In an analysis of children with NAS $(\mathrm{n}=2234)$ compared with a matched control group $(n=4330$, control $)$ and with other children from New South Wales, Australia ( $\mathrm{N}=598,265$, population), Oei et al. (2017) found that the mean test scores for literacy and numeracy were significantly lower for the NAS group in grade three (359 vs. control: 410 vs. population: 421) and that this deficit became progressively worse as the children aged. By middle school (grade 7), the scores for children with NAS were similar to grade 5 scores (deficit of two grade levels). In addition to the increased risk of not meeting minimum grade standards, which was independently associated with NAS (adjusted odds ratio $[\mathrm{aOR}], 2.5 ; 95 \%$ confidence interval $[\mathrm{CI}], 2.2-2.7)$, the researchers found three other factors that were associated with increased risk, but not as strongly: indigenous status, being male, and having parents with low educational attainment. Children who are born with an opioid dependency could have poor educational outcomes throughout their educational lifespans.

While the life outcomes for children born with NAS are represented in medical literature, it is more difficult to determine a clear representation for non-NAS children in homes impacted by opioids (Levine, 2018). Children in families impacted by substance use disorder (SUD) are underreported globally (Advisory Council on the Misuse of Drugs, 2003; Brundage \& Levine, 2019; Levine, 2018), which leads to less information and less formalized research about these vulnerable children. Brundage and Levine (2019) noted that many of the children in substanceimpacted families remain hidden from first-responders and care-givers unless there is a problem.

The children, however, do not remain hidden from teachers. They are in classrooms and possibly struggling with academics, behavioral, and social issues (Chapman, 2004; Cole et al., 2005; Fill et al., 2018; Herranz, Vílchez, Ledo, \& Sierra, 2014; Smith \& Wilson, 2016; Welby, 2019). Herranz et al. (2014) found that children $(\mathrm{N}=30)$ born in homes of mothers with opioid 
addiction had increased levels of depression and ADHD during their school trajectory and into adulthood. Those with diagnosed or classified ADHD were more likely to use cocaine regularly $(p=0.027 ; \mathrm{RR}=3.96 ; \mathrm{CI}: 95 \% ; 1.23-12.74)$, and regular cocaine use was also related to having been to a psychiatrist $(p=0.042)$. This longitudinal study followed children and reported on children who were not born addicted, but who were at higher risk— because of their home environment.

Welsh et al. (2018) described how the opioid epidemic has a social impact on schools that stems not only from possible family chaos and neglect but also from adolescents using substances. Welsh et al. reported that approximately 1.3 million adolescents (age 12-17) require treatment for substances, with many of these teens having struggles with school. There is a greater occurrence of mental health issues for adolescents who use substances compared to nonusing peers, and adolescent opioid use is correlated with anxiety-related diagnoses (Welsh et al., 2017).

Welby (2019) concluded that, for schools, the issues stemming from the opioid epidemic in communities of high impact would be experienced through special education, finances, teacher development, and curriculum support. These impacts will pertain not just to individual schools, but also to districts (Welby, 2019). Welby, who studied the impact of the opioid epidemic in three states, recommended further study in more geographic areas. This STS research is a step toward continuing the efforts to fill in the gaps nationally.

\subsubsection{Trauma Experienced by Children}

With SUD, there are higher rates of concurrent parental mental health problems, trauma history and PTSD, financial instability, interpersonal violence, social isolation, 
incarceration, and stress. All of these can intensify patterns of low parental involvement and monitoring of children's health and safety. (Stulac et al., 2019, p. 7)

Stulac et al. (2019) noted the strong association between households with SUD and issues of trauma for children in those households. While there is support for the notion that trauma becomes a part of a child's experience when raised in a home with SUD, defining trauma and understanding how it operates is essential for clarifying the impact on children. Similar to the section on opioids, this section will examine trauma in four ways: (a) brief background information; (b) definition in the research literature; (c) exploration of the impact on communities, families, and children; and (d) analysis of the effect on schools and educators. Brief background information. Because trauma has been shown to impair children's ability to learn and be successful socially in school (Cole et al., 2005), gaining a deeper understanding of trauma can support research for teachers and schools. Welby (2019) maintained that while all teachers required training in both the educational and socio-emotional needs of children traumatized by SUD, teachers themselves were experiencing vicarious trauma from supporting children. It is possible that the vicarious trauma referred to by Welby is due to the mechanism of trauma in children, regardless of the definition of trauma:

Trauma $\Rightarrow$ child $\Rightarrow$ child attends school (See Figure 2-2) 
Figure 2-2: School Trauma Factors and Process

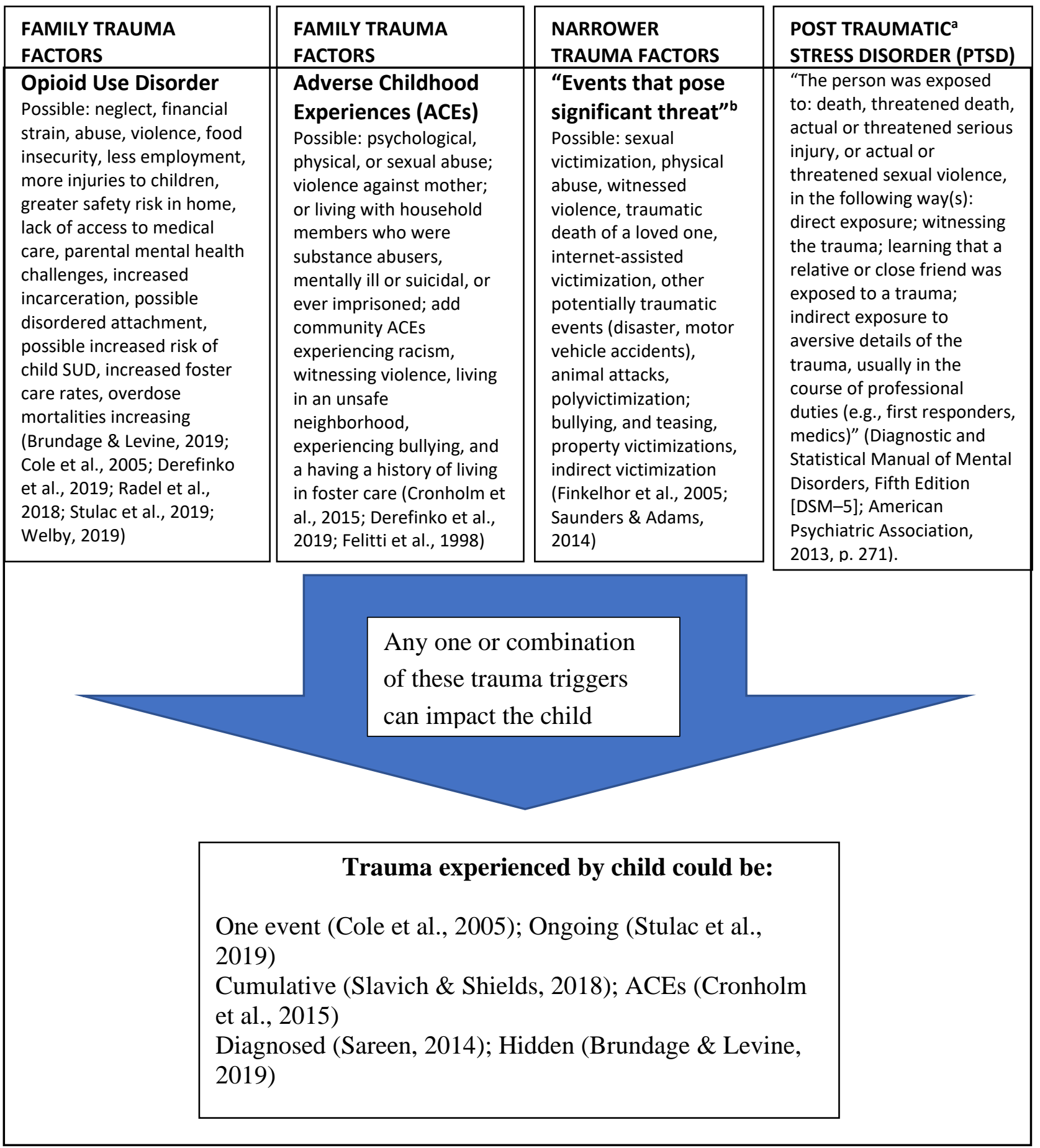

${ }^{a}$ To be diagnosed with PTSD, which is now in the Trauma and Stress-Related Disorders section of the DSM-V (Sareen, 2014), a patient must meet the diagnostic criteria of criterion A, one symptom (or more) from criterion B, one symptom (or more) from criterion $\mathrm{C}$, two symptoms (or more) from criterion $\mathrm{D}$, two symptoms (or more) from criterion E, and meet criteria $\mathrm{F}$ through $\mathrm{H}$.

${ }^{\mathrm{b}}$ American Psychological Association (2017, p. ES-3). 
Finkelhor, Ormrod, Turner, and Hamby (2005) found that, in a nationally representative sample of 2,030 children ages 2 to 17 years, $49 \%$ of the sample reported more than one type of direct or indirect victimization in the prior year. Additionally, an average of three victimizations was reported for any victimized child. This study implies that a survey focused on one type of trauma at a time, common for specific victimization studies like physical assaults or abducted children, will return a much lower number (Finkelhor, Hammer, \& Sedlak, 2002) than a survey focused on multiple types of victimization. For example, the Finkelhor et al. study (2005) included physical assaults, bullying, and teasing, sexual victimizations, child maltreatment, property victimizations, and indirect victimization (p. 22-23). These are also categories that are included in many definitions of trauma. For children, trauma needs to be addressed as part of a comprehensive approach to addressing the impact of opioids on children (Brundage \& Levine, 2019).

Definition and epidemiology of trauma. Before moving on to further discussion of the impact of trauma and children, it is important to define trauma and to locate the prevalence or incidence of trauma in the population of school children.

Definition. Krupnik (2019) noted that there is no agreement on the definition of trauma. There are numerous definitions of trauma represented in the research literature, located on a continuum from more generalized and subjective to more precise and objective (Krupnik, 2019; Saunders \& Adams, 2014). These definitions all have utility, depending on the research or practice goals. Still, they can also present a sense of muddle or grayness when trying to bracket or focus on precision of definition (Saunders \& Adams, 2014).

On the broader end of the spectrum, Cole et al. (2013) defined trauma as a "response to a highly stressful experience in which a person's ability to cope is dramatically undermined" (p. 
7). This definition is supportive of their goal for helping educators develop a trauma-informed lens: it assists teachers with increasing empathy for vulnerable students and decreasing blame or punitive responses for student behaviors. It serves the purpose of developing a "Trauma and Learning Policy Initiative" (Cole et al., 2013, p. 5) for all students, including those who have and have not experienced trauma.

On the other end of the continuum, Saunders and Adams (2014) link the need for precision in definition to "meaningful trauma research" (p. 1), with a call for accuracy. For example, there is consensus that violent stranger rape is counted as highly traumatic, but less consensus as to whether divorce or internet bullying counts as trauma-inducing (Saunders \& Adams, 2014). Likewise, there are also issues of boundaries within the definitions: researchers may disagree on the line between physical abuse and corporal punishment or between a parental disagreement and verbal abuse. Included in this more precise definition of trauma incidents are the following: sexual victimization, physical abuse, witnessed violence, traumatic death of a loved one, internet-assisted victimization, other potentially traumatic events (disaster, motor vehicle accidents, other accidents and animal attacks, and polyvictimization.

In between these two ends, the Adverse Childhood Experiences (ACEs) study describes traumatizing experiences utilizing some the precision of Saunders and Adams (2014), but some of the broadness noted by Cole et al. (2013). Both researchers refer to the ACEs study (Anda, Butchart, Felitti, \& Brown, 2010; Felitti et al., 1998). The original ACE study (Felitti et al., 1998) detailed the first survey results $(\mathrm{N}=13,494$ adults) with 9,508 responding $(70.5 \%$ response rate) regarding seven categories of adverse childhood experiences: psychological, physical, or sexual abuse; violence against mother; or living with household members who were 
substance abusers, mentally ill or suicidal, or ever imprisoned (p. 245), asked in 17 question prompts.

The results ranged from the least prevalent exposure category of evidence of criminal behavior in the household (3.4\%) to the most prevalent category of exposure of substance abuse in the household $(25.6 \%)$. One or more categories of exposure were affirmed by $52 \%$ of respondents. The probability of exposure to any additional category, for respondents affirming any single category of exposure, ranged from 65\%-93\% (median: 80\%). Felitti et al. (1998) noted a strong dose-response relationship between the number of ACEs endorsed and each of 10 health risk factors for the leading causes of death $(P<.001)$. The impact of ACEs is strong and cumulative (Felitti et al., 1998). The second wave of data from the original study increased the total respondents $(\mathrm{N}=17,337)$, and one or more categories of exposure were affirmed by $63.9 \%$ of respondents (Centers for Disease Control and Prevention, 2020a).

One critique of the ACEs study is that it relied on data collected primarily in the home and data from a cohort of primarily White, middle- to upper-class participants (Cronholm et al., 2015). The Philadelphia (PHL) ACEs Survey, a follow-up to the Philadelphia Health Management Corporation (PHMC)'s 2012 Southeastern Pennsylvania Household Health Survey ( $\mathrm{N}=413,000)$, polled a total of 1,784 Philadelphia residents (aged 18 or older). Respondents were contacted to complete an additional interview containing questions about Conventional and Expanded ACEs. The additional questions included prompts about stressors outside the household (i.e., Expanded ACEs) including “experiencing racism, witnessing violence, living in an unsafe neighborhood, experiencing bullying, and a having a history of living in foster care" (Cronholm et al., 2015, p. 355). Several other differences included not assessing parental divorce and more detailed content of emotional and physical neglect. Except for sexual abuse, emotional 
neglect, and physical neglect (which were reported less frequently in the PHL ACEs Survey sample, $p<0.001)$, the PHL ACEs survey respondents reported higher rates for all conventional ACEs $(p<0.001)$ compared to the original Kaiser sample. Participants in the PHL ACEs survey described high rates of witnessing community violence $(40.5 \%)$, racial discrimination $(34.5 \%)$, feeling that their neighborhood was unsafe $(27.3 \%)$, the experience of bullying growing up $(8.0 \%)$, and experience with the foster care system while growing up (2.5\%). Ordinal regression showed that gender, race, and poverty were associated with a higher risk for PHL ACEs (community/expanded ACEs), but not with a higher risk for Conventional ACEs (Kaiser study). With 95\% CI for all categories, the OR for males: Kaiser 1.27 (0.97, 1.67), PHL 2.05 (1.53, 2.75); Black or African American: Kaiser 0.89 (0.68, 1.16), PHL 3.07 (2.31, 4.08); Hispanic or Latino: Kaiser $1.21(0.49,2.96)$, PHL 5.93 (1.77, 19.90); Asian or Pacific Islander: Kaiser 0.83 (0.34, 2.02), 3.93 (1.19, 12.94); and poverty: Kaiser 1.20 (0.85, 1.69), PHL $1.51(1.03,2.20)$. These findings support the notion that expanding the conventional ACE measure could be a more accurate assessment of adversity than conventional measures of household adversity. Finally, Derefinko et al. (2019) reflected that ACEs are understudied in the area of the opioid epidemic.

Krupnik (2019) advocated for a hybrid model of traumatic stress response to bridge the expanse between the more structured and rigid definitions of traumatic response and the inclusive subjective ones. In this model, stress is divided into three response groups: (a) normative (healthy) stress response, (b) pathogenic stress response, and (c) traumatic stress response. In this traumatic stress response state, the person is not able to self-regulate their internal states. Traumatic stress response differs from the normative stress response where the person can return to their initial state before the stressor. The traumatic state also differs from the pathogenic state in that in pathogenic stress response, the person transitions to a different and 
less adaptive state than the beginning state. Krupnik noted that people with a less developed sense of self-regulation, like children, are particularly susceptible to traumatization. This breakdown of self-regulation is referenced as a decrease in executive functioning, which is detected by professionals and researchers working with children who have been traumatized (Cole et al., 2013).

Post-traumatic Stress Disorder (PTSD). For this dissertation, the definition of trauma needs to include a discussion of post-traumatic stress disorder (PTSD). The American Psychological Association (2017) defines trauma as stemming from an event that is threatening in some way physically, emotionally, or psychologically. The significant threat can be to the safety of the traumatized person or can be to the friends or loved ones of the traumatized person. Finally, this threat is both overwhelming and shocking. This rigorous definition of trauma contrasts with some of the broader, less precise definitions of trauma (Krupnik, 2019). People can be exposed to traumatic events in one (or more) of the following ways: "1) direct experiencing; 2) witnessing, in person; 3) learning that the traumatic event(s) occurred to a family member or someone else in close relationship; 4) experiencing repeated or extreme exposure to aversive details of the traumatic event(s) (common in first responders and emergency personnel)" (American Psychological Association, 2017, p. 6). According to the 5th edition of the Diagnostic and Statistical Manual of Mental Disorders, DSM-5, (American Psychiatric Association, 2013), PTSD is defined as composed of four groups of symptoms, which include the following: (1) memories of trauma that are intrusive and recurrent, (2) avoidance of trauma-related stimuli, (3) changes in mood that are negative or numbing or changes in thoughts about the trauma, and (4) reaction and arousal changes. In the DSM-5, the 
PTSD subtype has been added for preschool children, which has increased the chances of diagnosing PTSD in children (Kolaitis, 2017).

Epidemiology. Although types of traumatic life events vary between ones that threaten great injury, engage hopelessness, or cause a sense of loss, the rate of exposure for children and adolescents holds steady at around two-thirds or 66\% (La Greca et al., 2008). Copeland, Keeler, Angold, and Costello, (2007) noted that $67.8 \%$ of children in their longitudinal trauma and PTSD study $(\mathrm{N}=1420)$ affirmed they had experienced one or more traumatic events. La Greca et al. (2008) detailed the range in the prevalence of the following: youth witnessing violence (39\%$85 \%)$ and youth exposed to sexual abuse (25\%-43\%).

Copeland et al. (2007) noted that, of the $67.8 \%$ of children who experienced traumatizing events, only $13.4 \%$ of the children developed any sort of posttraumatic stress symptoms. Adolescence was more commonly associated with trauma than childhood $(z=1.99 ; p=.05)$. This prevalence is slightly lower than other reports: Kolaitis (2017) maintained about $16 \%$ of traumatized children and adolescents would develop PTSD; Guterman et al. (2016) placed the percentage at close to $15 \%$. But La Greca et al. (2008) reported that "nearly all children and adolescents express some kind of distress or behavioral change in the acute phase of recovery from a traumatic event" (p. 2). While nearly all children will react to a traumatic event, a much smaller percentage will then develop PTSD.

Costello, Erkanli, Fairbank, and Angold, (2002) found gender differences among children's vulnerability factors that increased risk for traumatic events. Boys had increased risk associated with a parental history of mental illness $(\mathrm{OR}=2.1,95 \% \mathrm{CI}=1.5-3.0, p<.001)$. For girls, being from families with a history of mental illness increased the likelihood of exposure to traumatic events ( $\mathrm{OR}=3.2,95 \% \mathrm{CI}=2.3-4.5, p<.001)$. So for both boys and girls, mental 
illness in family history doubled the risk of exposure. In addition, girls with parents who had a criminal record $(\mathrm{OR}=1.7,95 \% \mathrm{CI}=1.1-2.6, p=.05)$ and girls from high poverty homes or homes with low education $(\mathrm{OR}=2.3,95 \% \mathrm{CI}=1.5-3.5, p<.001)$ were also at increased risk.

Impact of trauma on children. Saunders and Adams (2014) found that two characteristics were common, regardless of age, gender, and type of trauma. First, as children age, trauma accumulates, and this burden becomes more accumulative. Children have more experiences as they age, which increases their exposure. Second, the teen years are particularly dangerous for children, exposing them to many new types of traumatic events. The sexual assault risk for girls is three to four times greater than for boys (Saunders \& Adams, 2014).

The cumulative load of stress, adversity, and trauma on the lifespan were also supported by the validated work ( $\mathrm{N}=205)$ of Slavich and Shields (2018) who found that the total lifetime stressor count was strongly correlated with the respondents' childhood adversity (CTQ-SF) total score $(r=.552, p<.001)$. In addition, the participants' lifetime stressor count was significantly associated with the following: more self-reported current physical health complaints $(r=.321, p$ $<.001)$; more physical health complaints $(r=.469, p<.001)$; worse sleep quality over the past month $(r=.493, p<.001)$; and poorer executive function $(r=.185, p=.008)$. Although those were self-reports, the researchers also looked at doctor-diagnosed issues. The lifetime stressor count was significantly related to the following doctor-diagnosed issues: general health problems (risk ratio $[\mathrm{RR}]=1.026,95 \%$ confidence interval $[\mathrm{CI}]=1.019-1.034, p<.001)$ and autoimmune disorders $(\mathrm{RR}=1.034,95 \% \mathrm{CI}=1.017-1.051, p<.001)$. Each addition life stressor increased the likelihood by $2.6 \%$ of participants be diagnosed with a major health condition and $3.4 \%$ with an autoimmune disorder. Although the youngest participant in the study was 19 years old, Slavich and Shields (2018) were assessing cumulative stress by using the Childhood Trauma 
Questionnaire-Short Form as one of their instruments (CTQ-SF; Bernstein et al., 2013), asking about childhood trauma.

Van der Kolk (2005) reported that approximately $80 \%$ of child maltreatment is due to home or parents. Van der Kolk described complex trauma as "the experience of multiple, chronic and prolonged, developmentally adverse traumatic events, most often of an interpersonal nature (e.g., sexual or physical abuse, war, community violence) and early-life onset” (p. 402). These relate to the child's home because the exposures to the traumatic events often occur within the caregiving system or home of the child, beginning in early childhood, and include neglect (physical, emotional, and educational), as well as child maltreatment. This type of chronic trauma experience has grave implications for educators, as discussed in the next section.

Effect of children's trauma on schools and educators. Because children who are exposed to the impact of ongoing, serious, and cumulative trauma are vulnerable to the impact of subsequent trauma (La Greca et al., 2008), educators need to be aware of this impact (Cole et al., 2005; Cole et al., 2013), especially in communities of high stress. Derefinko et al. (2019) linked ACEs to increased risk of opioid use, increased use at a younger age, and increased opioid relapse. ACEs are also associated with an increase in risky behaviors and a decrease in academic achievement (Normile et al., 2018). For teachers, then, work with students from communities with high opioid use may include a wide range of issues that impact the teaching day.

Van der Kolk (2005) described the implications of chronic trauma for children: deficits in self-regulation, poor affect and impulse control, aggression against others and even directed against the self, distrust and suspiciousness, and difficulties with social isolation. Children can have "alterations in states of consciousness" (Van der Kolk, 2005, p. 404), which could include dissociative events, flashbacks, and attention issues. Cause-and-effect thinking can be impacted, 
and children can have a sense of continual threat. However, Van der Kolk cautioned that not all traumatized children have PTSD, reflecting that "The diagnosis of PTSD is not developmentally sensitive and does not adequately describe the effect of exposure to childhood trauma on the developing child" (p. 405). Streeck-Fischer and Van der Kolk (2000) emphasized that, for children with complex trauma, teachers can be seen as part of the problem —as re-enactors of the trauma.

Traumatic stress has an impact on academic performance and children's behavior and relationships at school (Cole et al., 2005; Cole et al., 2013; Goodman, Miller, \& West-Olatunji, 2012; Ridgard, Laracy, Dupaul, Shapiro, \& Power, 2015). Goodman et al. (2012) assessed for traumatic stress and academic achievement in $5^{\text {th }}$-grade students $(\mathrm{N}=11,820)$ and found the following: students with low SES had higher levels of traumatic stress; as student traumatic stress increased, absences increased; students with traumatic stress had a lower reading achievement mean score than students without trauma (11.932 points lower, $p .001)$; students with traumatic stress had a lower math achievement mean score than students without trauma (10.883 points lower, $p .001)$; and students with traumatic stress had a lower science achievement mean score than students without trauma (5.689 points lower, $p$.001). For students with traumatic stress, academic achievement can be negatively impacted sd a result of "their inability to process information, meaningfully distinguish between threatening and nonthreatening situations, form trusting relationships with adults, and modulate their emotions" (Cole et al., 2005, p. 21). Ridgard et al. (2015) noted that because trauma exposure negatively impacts children's school functioning, school professionals can support children by adopting a trauma-informed approach that begins with realizing the widespread nature of trauma and recognizing the impact of trauma. 


\subsubsection{Secondary Traumatic Stress Framework}

The framework of secondary traumatic stress is built on a clear understanding of both the definition of secondary stress and the diagnostic framework. First, various types of stress will be explored, with secondary stress delineated from these. Next, a definition will be distilled for STS. Finally, the diagnostic framework will be examined with particular attention to the PTSD diagnostic elements that distinguish STS from other types of stress.

Definition and types of secondary stress. Understanding the way that teachers may experience STS in communities of high stress requires a clear comprehension of the nuances of the meaning of STS and, in particular, how the diagnostic features are distinct from similar stress- and trauma-based experiences. Although STS definition and diagnosis proceed from a medicalized paradigm, neither are entirely straightforward; therefore, both the definition and diagnosis will also be examined.

Multiple definitions for similar constructs. Stamm (2010) reflected that caring for others within the realm of professional duties can involve both positive and negative aspects. Positive caring can bring a sense of satisfaction or self-efficacy; negative aspects of caring include a sense of fatigue (Stamm, 2010). This fatigue has been identified in the research literature by several different names, with overlapping identifying features. Three terms are associated with the adverse effects of caring for those with traumatic event exposure: vicarious traumatization (VT), compassion fatigue (CF), and secondary traumatic stress (STS). The attempt to locate one agreed-upon definition for STS is a challenging research task, made more arduous by the paucity of independently peer-reviewed studies (Devilly, Wright, \& Varker, 2009).

Discriminating STS from other definitions. Sprang, Ford, Kerig, and Bride (2019) noted that there are still some inconsistencies in the way that STS is defined and measured. Based on 
the numerous definitions, it might be useful to evaluate how STS compares to similarly situated reactions (see Table 2-2).

Table 2-2: Variations in STS-related Definitional Components

\begin{tabular}{|c|c|}
\hline Concept & Definition Components \\
\hline Secondary traumatic stress (STS) & $\begin{array}{l}\text { "Experienced an event outside the range of usual } \\
\text { human experiences that would be markedly } \\
\text { distressing to almost anyone," (Figley, 1995, p. 8), } \\
\text { such as sudden tragedy which could destroy the } \\
\text { traumatized person's (TP) environs or a severe } \\
\text { threat to TP. It can develop suddenly, in contrast to } \\
\text { burnout (Figley, 1995; Rzeszutek, Partyka, \& } \\
\text { Gołąb, 2015); this is described as a critical incident } \\
\text { (Salston \& Figley, 2003). Similar to CF, but more } \\
\text { exact terminology (Figley, 1995); STS is one } \\
\text { element of CF (Stamm, 2010); psychological } \\
\text { symptoms more present in STS than schema } \\
\text { interruption in VT (Baird \& Kracen, 2006); amount } \\
\text { of exposure to clients, caseload, and cumulative } \\
\text { impact increases the likelihood of STS (Baird \& } \\
\text { Kracen, 2006; Myers \& Cornille, 2002; Wee \& } \\
\text { Myers, 2002); Most directly linked to the PTSD } \\
\text { diagnostic framework (Devilly et al., 2009); }\end{array}$ \\
\hline Vicarious trauma (VT) & $\begin{array}{l}\text { Because of being exposed to the traumatic material, } \\
\text { the respondent develops a change of view about } \\
\text { themselves or the world (McCann \& Pearlman, } \\
\text { 1990); VT's harmful impact work to disrupt } \\
\text { schemas in five areas: safety, trust, esteem, } \\
\text { intimacy, and control (Baird \& Kracen, 2006); some } \\
\text { evidence for a relationship between professional } \\
\text { having a personal history of trauma linked to the } \\
\text { development of VT, as cited in research synthesis } \\
\text { (Baird \& Kracen, 2006) }\end{array}$ \\
\hline Compassion fatigue (CF) & $\begin{array}{l}\text { Terminology preferred by some helping } \\
\text { professionals, like doctors (Nimmo \& Huggard, } \\
\text { 2013); some researchers support the notion that CF } \\
\text { can be used interchangeably with STS (Figley, }\end{array}$ \\
\hline
\end{tabular}




\begin{tabular}{|c|c|}
\hline & $\begin{array}{c}\text { 1995); reduced ability to remain empathic to or } \\
\text { interested in clients due to emotions and emotional } \\
\text { response (Adams, Boscarino, \& Figley, 2006); loss } \\
\text { of energy, similar in effect to burnout (Stamm, } \\
\text { 2010); compassion satisfaction could work as a } \\
\text { protective factor (Figley, 2002); }\end{array}$ \\
\hline Burnout & $\begin{array}{c}\text { Stems from job stress (Maslach \& Jackson, 1981); } \\
\text { experience exhaustion mentally, emotionally, and } \\
\text { physically because of involvement in long-term } \\
\text { situations with high demands (Pines \& Aronson, } \\
1988 \text { ); this is a more gradual process (Cherniss, } \\
1980 ; \text { Courage \& Williams, 1986; Pines, Aronson, } \\
\text { \& Kafry, 1981) }\end{array}$ \\
\hline Post-traumatic stress disorder (PTSD) & $\begin{array}{c}\text { "Experienced an event outside the range of usual } \\
\text { human experiences that would be markedly } \\
\text { distressing to almost anyone," (Figley, 1995, p. 8), } \\
\text { such as sudden environmental tragedy or a severe } \\
\text { threat to self. }\end{array}$ \\
\hline
\end{tabular}

The definition of STS is often defined by the researcher, contextually driven by the topic of research, and overlapping in features with related constructs, particularly CF, VT, and burnout. There are dual emphases in STS, which will be valuable in the discussions of teachers in communities of high stress. First, the primary stressor is experienced in the environment. Second, STS results from helping an affected person (Bride et al., 2004), especially in a way that causes a "disturbance of one's emotions and/or cognitions as a result of experiencing the effect of trauma on others" (Motta, 2012, p. 257). This definition means that the trauma experienced in STS is not a result of internalized conditions like anxiety or depression, but instead instigated from interaction with external conditions. Nor is STS a result of the helping professional's trauma, but rather the outcome of caring for one who has been traumatized (Stamm, 1999). There is some evidence that the development of STS is not linked to a previous 
history of trauma in the caregiver (Baird \& Kracen, 2006). The person who experiences STS is in a supportive role, sometimes just for a brief period and sometimes for a sustained time, which supports the contention that STS can develop quickly (Figley, 1995), and even after one exposure (VanBergeijk \& Sarmiento, 2006).

Definition of secondary traumatic stress. Figley (1999) defined secondary traumatic stress as "the natural, consequent behaviors and emotions resulting from knowledge about a traumatizing event experienced by a significant other. It is the stress resulting from helping or wanting to help a traumatized or suffering person" (p. 10). Cummings, Singer, Hisaka, and Benuto (2018) contrasted STS with vicarious trauma and burnout by describing the acute onset of STS compared to the more prolonged onset for burnout and vicarious trauma. Salston and Figley (2003) noted that STS could develop after a "critical incident" (p. 171), compared to the effects of vicarious trauma which "develop over time” (p. 169). Branson (2019) described, "STS is more appropriate for professionals who may be shocked and overwhelmed by someone's trauma. Additionally, STS can be acute, occurring suddenly after one encounter” (p. 5), as opposed to vicarious trauma, which develops slowly. Likewise, burnout is a construct that develops over time, with gradually emerging symptoms as the common etiology (Maslach, 2003). STS has been associated with a more sudden onset of symptoms (Benson \& Magraith, 2005; Figley, 1999; Rzeszutek et al., 2015; Salston \& Figley, 2003). It should be noted that STS might not occur quickly for everyone, but that a sudden onset is possible. There are differences between PTSD and STS (Mordeno, Go, \& Yangson-Serondo, 2017), so the assessment for STS should be completed with a tool that is designed for STS.

Diagnostic features of STS. The diagnosis of STS, primarily when assessed by the Secondary Traumatic Stress Scale (STSS; Bride et al., 2004), relies on the diagnostic features of 
PTSD, which, in turn, requires an understanding of trauma. Although the topic of trauma in children has been examined above in this literature review, this current discussion involves the DSM-relevant definition to locate the characteristics of STS. By placing the discrete attributes of PTSD within the conceptualization of trauma, STS can be more clearly understood.

Trauma. DSM-V (American Psychiatric Association, 2013) defines trauma as the following:

The directly experienced traumatic events in Criterion A include, but are not limited to, exposure to war as a combatant or civilian, threatened or actual physical assault (e.g., physical attack, robbery, mugging, childhood physical abuse), threatened or actual sexual violence (e.g., forced sexual penetration, alcohol/drug-facilitated sexual penetration, abusive sexual contact, noncontact sexual abuse, sexual trafficking), being kidnapped, being taken hostage, terrorist attack, torture, incarceration as a prisoner of war, natural or human-made disasters, and severe motor vehicle accidents. (p. 274)

The manual (American Psychiatric Association, 2013) continues with parsing medical trauma, trauma for young children, and witnessed events,

Indirect exposure through learning about an event is limited to experiences affecting close relatives or friends and experiences that are violent or accidental (e.g., death due to natural causes does not qualify). Such events include violent personal assault, suicide, serious accident, and serious injury. The disorder may be especially severe or long-lasting when the stressor is interpersonal and intentional (e.g., torture, sexual violence). (p. 275) The definition of trauma that is experienced indirectly is salient for the understanding of the mechanism of STS. 
Diagnosis of PTSD. The determination of STS is formed from the working components of the diagnostic features of post-traumatic stress disorder (PTSD). Much of the current work for STS utilizes the information from DSM-IV (Bride et al., 2004; Bride, 2007), but there is a new update to the DSM-V (American Psychiatric Association, 2013), which has made some changes to the criteria. The DSM-IV guidelines for PTSD "recognizes the importance of contextualizing stressful experiences" (Stamm, 1999).

Diagnostic criteria for PTSD. Although the DSM-V, Code 309.81 (American Psychiatric Association, 2013, p. 217) details the most updated criteria for the diagnosis of PTSD, the STSS was based on the requirements of an earlier version, the DSM-IV-TR (American Psychiatric Association, 2000). Secondary stress, as evaluated by the STSS (Bride et al., 2004), requires an understanding, then, of the DSM-IV-TR diagnostic criteria (see Table 2-3).

Table 2-3: Comparison of DSM-IV-TR Diagnostic Criteria and STS Subscale Indicators

\begin{tabular}{|c|c|c|c|}
\hline $\begin{array}{c}\text { Criteria } \\
\text { Identifier }\end{array}$ & Criteria $^{a}$ & Features $^{a}$ & $\begin{array}{c}\text { Secondary } \\
\text { Traumatic Stress } \\
\text { Indicators }\end{array}$ \\
\hline $\mathbf{A}$ & $\begin{array}{l}\text { The person has } \\
\text { been exposed to } \\
\text { a traumatic } \\
\text { event in which } \\
\text { both of the } \\
\text { following were } \\
\text { present: }\end{array}$ & $\begin{array}{l}\text { (1) The person experienced, witnessed, or } \\
\text { was confronted with an event or events } \\
\text { that involved actual or threatened death or } \\
\text { serious injury, or a threat to the physical } \\
\text { integrity of self or others. } \\
\text { (2) The person's response involved } \\
\text { intense fear, helplessness, or horror. Note: } \\
\text { In children, this may be expressed instead } \\
\text { by disorganized or agitated behavior. }\end{array}$ & $\begin{array}{l}\text { Not used by the } \\
\text { STSS for the } \\
\text { indication of STS } \\
\text { (Bride, 2007) }^{\mathrm{b}}\end{array}$ \\
\hline B & $\begin{array}{l}\text { The traumatic } \\
\text { event is } \\
\text { persistently } \\
\text { reexperienced in } \\
\text { one (or more) of }\end{array}$ & $\begin{array}{l}\text { (3) Recurrent and intrusive distressing } \\
\text { recollections of the event, including } \\
\text { images, thoughts, or perceptions. Note: In } \\
\text { young children, repetitive play may occur } \\
\text { in which themes or aspects of the trauma } \\
\text { are expressed. }\end{array}$ & $\begin{array}{l}\text { Relates to the } \\
\text { intrusion subscale } \\
\text { of the STSS } \\
\text { (Bride et al., } \\
\text { 2004) and }\end{array}$ \\
\hline
\end{tabular}




\begin{tabular}{|c|c|c|c|}
\hline & $\begin{array}{l}\text { the following } \\
\text { ways: }\end{array}$ & $\begin{array}{l}\text { (4) Recurrent distressing dreams of the } \\
\text { event. Note: In children, there may be } \\
\text { frightening dreams without recognizable } \\
\text { content. } \\
\text { (5) Acting or feeling as if the traumatic } \\
\text { event were recurring (includes a sense of } \\
\text { reliving the experience; illusions, } \\
\text { hallucinations, and dissociative flashback } \\
\text { episodes, including those that occur on } \\
\text { awakening or when intoxicated). Note: In } \\
\text { young children, trauma-specific } \\
\text { reenactment may occur. } \\
\text { (6) Intense psychological distress at } \\
\text { exposure to internal or external cues that } \\
\text { symbolize or resemble an aspect of the } \\
\text { traumatic event. } \\
\text { (7) Physiological reactivity on exposure to } \\
\text { internal or external cues that symbolize or } \\
\text { resemble an aspect of the traumatic event. }\end{array}$ & $\begin{array}{c}\text { instruction } \\
\text { symptoms of STS }\end{array}$ \\
\hline $\mathrm{C}$ & $\begin{array}{c}\text { Persistent } \\
\text { avoidance of } \\
\text { stimuli } \\
\text { associated with } \\
\text { the trauma and } \\
\text { numbing of } \\
\text { general } \\
\text { responsiveness } \\
\text { (not present } \\
\text { before the } \\
\text { trauma), as } \\
\text { indicated by } \\
\text { three (or more) } \\
\text { of the following: }\end{array}$ & $\begin{array}{l}\text { (8) Efforts to avoid thoughts, feelings, or } \\
\text { conversations associated with the trauma } \\
\text { (9) Efforts to avoid activities, places, or } \\
\text { people that arouse recollections of the } \\
\text { trauma } \\
\text { (10) Inability to recall an important aspect } \\
\text { of the trauma } \\
\text { (11) Markedly diminished interest or } \\
\text { participation in significant activities } \\
\text { (12) Feeling of detachment or } \\
\text { estrangement from others } \\
\text { (13) Restricted range of affect (e.g., } \\
\text { unable to have loving feelings) } \\
\text { (14) Sense of a foreshortened future (e.g., } \\
\text { does not expect to have a career, marriage, } \\
\text { children, or a normal lifespan) }\end{array}$ & $\begin{array}{l}\text { Relates to the } \\
\text { avoidance } \\
\text { subscale of the } \\
\text { STSS (Bride et } \\
\text { al., 2004) and } \\
\text { avoidance } \\
\text { symptoms of STS }\end{array}$ \\
\hline D & $\begin{array}{c}\text { Persistent } \\
\text { symptoms of }\end{array}$ & $\begin{array}{l}\text { (1) Difficulty falling or staying asleep } \\
\text { (2) Irritability or outbursts of anger }\end{array}$ & $\begin{array}{l}\text { Relates to the } \\
\text { arousal subscale }\end{array}$ \\
\hline
\end{tabular}




\begin{tabular}{|c|c|c|c|}
\hline & $\begin{array}{l}\text { increased } \\
\text { arousal (not } \\
\text { present before } \\
\text { the trauma), as } \\
\text { indicated by two } \\
\text { (or more) of the } \\
\text { following: }\end{array}$ & $\begin{array}{l}\text { (3) Difficulty concentrating } \\
\text { (4) Hypervigilance } \\
\text { (5) Exaggerated startle response }\end{array}$ & $\begin{array}{c}\text { of the STSS } \\
\text { (Bride et al., } \\
\text { 2004) and arousal } \\
\text { symptoms of STS }\end{array}$ \\
\hline $\mathbf{E}$ & $\begin{array}{l}\text { Duration of the } \\
\text { disturbance } \\
\text { (symptoms in } \\
\text { Criteria B, C, } \\
\text { and D) is more } \\
\text { than } 1 \text { month. }\end{array}$ & & $\begin{array}{l}\text { Not used by the } \\
\text { STSS for the } \\
\text { indication of STS } \\
\text { (Bride, 2007) }^{\mathrm{b}}\end{array}$ \\
\hline $\mathbf{F}$ & $\begin{array}{l}\text { The disturbance } \\
\text { causes clinically } \\
\text { significant } \\
\text { distress or } \\
\text { impairment in } \\
\text { social, } \\
\text { occupational, or } \\
\text { other important } \\
\text { areas of } \\
\text { functioning. }\end{array}$ & & $\begin{array}{l}\text { Not used by the } \\
\text { STSS for the } \\
\text { indication of STS } \\
\text { (Bride, 2007) }^{\mathrm{b}}\end{array}$ \\
\hline
\end{tabular}

${ }^{\mathrm{a}}$ Adapted from the Center for Substance Abuse Treatment (2009). ${ }^{\mathrm{b}}$ Regarding the exclusion of criteria A, E, and F, Bride (2007) noted that this is "typical of many trauma measures as the B, C, and D criteria are considered to be the core symptoms of PTSD” (p. 67).

This PTSD diagnosis is essential because the impact of STS on the helper often mirrors the trauma symptoms seen in the traumatized population (Bride, Radey, \& Figley, 2007). Ireland and Huxley (2018) reflected that unaddressed PTSD symptoms could continue to develop and intensify, even to the point where therapists "may begin experiencing similar symptoms to their clients" (p. 143). Although PTSD is the diagnostic criteria that would be useful for trauma client identification, the symptomology presents in the caregiver or helper with STS. 
Defining features of STS. Because secondary traumatic stress results from helping or wanting to help another who has been traumatized (Figley, 1999; Bride et al., 2004; Bride, 2007), it also has symptoms that parallel those that are noted in victims of trauma (Chrestman, 1999). These include intrusion, avoidance, and arousal symptoms (Figley, 1999). A comparison of intrusion, avoidance, and arousal is necessary to understand STS fully.

Intrusion. Intrusion is highlighted as re-experiencing, either as intrusive thoughts, recurrent or distressing dreams in which the trauma is replayed, or reliving the event in the form of flashbacks, recurrences, or illusions. Intrusion symptoms can include distress when reminded of the event, or a physiological reaction when confronted with a reminder of the event in the form of internal or external cues (American Psychiatric Association, 2000; Substance Abuse and Mental Health Services Administration, 2016). When Bride (2007) first examined the prevalence of STS in social workers $(\mathrm{N}=282)$ using the Secondary Traumatic Stress Scale (STSS; Bride et al., 2004), he found individual intrusion symptoms at this rate: intrusive thoughts about clients (40.5\%); experiencing psychological distress when reminded of work with traumatized clients (19.1\%); experiencing physiological reactions (like heart-pounding) when reminded of work with traumatized clients (12.4\%); disturbing dreams about clients (5.8\%); and sense of reliving the trauma experienced by the client $(5.0 \%)$.

Avoidance. The hallmarks of avoidance involve the effort to avoid thoughts or feelings that are associated with the trauma, along with avoiding activities, places, and people that also might elicit reminders of the trauma. Avoidance is also indicated by the inability to recall important details about the trauma, less interest in significant events, detachment, and a restricted range of emotions or affect. Finally, avoidance can trigger a numbed sense of future expectation (American Psychiatric Association, 2000; Substance Abuse and Mental Health Services 
Administration, 2016). Bride (2007) found that social workers reported the following avoidance symptoms: avoidance of some clients (31.6\%), a sense of discouragement about the future (28.0\%), a feeling of emotional numbness (25.9\%), diminished activity level (25.5\%), reduced interest in being around others (22.3\%), difficulty recalling information related to work with traumatized clients (14.9\%), and avoidance of people, places, and things that reminded them of their work $(10.9 \%)$.

Arousal. Arousal symptoms are characterized by persistent anxiety-like symptoms that were not noticed prior to trauma exposure. These symptoms include difficulty with sleep (either falling asleep or staying asleep), irritability or outbursts of anger, concentration difficulties, hypervigilance, and an extreme or exaggerated startle response (American Psychiatric Association, 2000; Substance Abuse and Mental Health Services Administration, 2016). For Bride's (2007) study, respondents affirmed irritability at (27.7\%), difficulty concentrating (27.0\%), difficulty with sleeping (24.4\%), a sense of hypervigilance by expecting something bad to happen (13.8\%), and feeling jumpy or easily startled (12.1\%).

Measurement of STS. To measure the construct of STS in the above studies, researchers relied on self-report survey research and the use, in particular, of the Secondary Traumatic Stress Scale (STSS; Bride et al., 2004). Before examining the STSS in the next section, the discussion of the measurement of STS will conclude with a few observations about survey research, its limitations, and other measures that are useful for assessing STS.

Survey research. Surveys can be an effective means of gathering information and measure for social and educational research (Ruel, Wagner, \& Gillespie, 2016). With the use of online administration, survey research is "pervasive in the modern Western world" (Ruel et al., 2016, p. 2), is an important research tool, provides flexibility for a wide number of fields, and 
can be useful for generalizability (Ruel et al., 2016). For accuracy, though, for issues like stress, trauma, and PTSD, the use of surveys and checklists has some caveats. McDonald and Calhoun (2010) described that although over $90 \%$ of adults have experienced a traumatic event, $8 \%$ or more will develop full-blown PTSD. With the use of PTSD checklists, it might be possible to identify characteristics of a construct or determine prevalence; self-reports could then be paired with a diagnostic interview for full identification of PTSD (McDonald \& Calhoun, 2010). In this way, self-report survey research can help with identification or prevalence estimates, but care should be taken not to substitute self-report surveys for stand-alone diagnostic measures.

In designing survey research, Draugalis, Coons, and Plaza (2008) recommended ten guidelines for best practices, ranging from a clearly defined research question, representative sampling, well-balanced design between cost and error, a clear and full description of the instrument, pretesting the instrument and utilizing quality control, a generalizable response rate along with appropriate statistical techniques, ethical oversight, and author transparency to aid replication. Weigold, Weigold, and Russell (2013) found, in two separate paired studies $(\mathrm{N}=$ 256) and $(\mathrm{N}=203)$, that paper-and-pencil data collection has shown equivalence to internet data collection. The survey for this study, the STSS, was selected based on survey information from Draugalis et al. (2008) and Weigold et al. (2013).

Limitations of survey research. There are some limitations with self-reported data. Chan (2009) noted that there are different types of data self-reported by respondents; data such as demographic data appear to be less problematic than personality data due to personality data (like neuroticism) being value-laden. Chan identified four limitations or problems with selfreported data: construct validity, difficulty with correlations, the impact of social desirability, and the status of non-self-reported data. Because the construct in this study is secondary stress, 
and because the survey is confidential, these limitations are minimized. While it is important to be knowledgeable about the limitations of self-reported data, it is also important to acknowledge that self-reported data are not inherently flawed.

Additional measures for STS survey research. Although the STSS is used to assess secondary traumatic stress, it is by no means the only instrument developed to do so. Stamm (1996) reviewed 98 instruments for the measurement of stress, trauma, and adaptation, which reflects the proliferation of instruments for trauma and stress. To evaluate the fit of the STSS for this research study with K-12 public school teachers, consideration of when this particular instrument is used — especially compared to other instruments — will be informative. Nimmo and Huggard (2013) delineated how different measurements are needed to measure the three different constructs of compassion fatigue, vicarious trauma, and secondary traumatic stress. However, each of the constructs describes a different aspect of caring, and, therefore, utilizes different measures.

Without going into detail about the other instruments, the complexity of constructs such as compassion fatigue, vicarious trauma, and secondary traumatic stress has given rise to numerous measures that are tailored for those symptoms. It is useful to briefly note some of the measures before moving on to a more full-throated discussion of the STSS for secondary stress. For compassion fatigue, researchers will often use the Compassion Fatigue/Satisfaction Self Test (CFST; Figley, 1996; Figley \& Stamm, 1996), Compassion Satisfaction and Fatigue Test (Stamm, 2002), Compassion Fatigue Scale (Gentry, Baranowsky, \& Dunning, 2002), Compassion Fatigue - Short Scale (Adams et al., 2006), or the Professional Quality of Life scale developed by Stamm (ProQOL; 2005, 2009). For vicarious trauma, two common instruments 
include the Traumatic Stress Institute Belief Scale - Revision L (TSI-BSL; Jenkins \& Baird, 2002) and Traumatic Stress Institute Life Events Checklist (TSI-LEC; Bride et al., 2007).

Many studies have utilized the STSS because of its congruence with PTSD diagnostic criteria (Bride, Smith Hatcher, \& Humble, 2009). Compassion fatigue and vicarious trauma do not share this same congruence. The STSS, however, is also used in conjunction with other surveys. In this way, researchers are exploring relationships among constructs or variables. For example, Bride and Figley (2009) used the STSS with the Compassion Fatigue-Short Scale (CFSS; Adams et al., 2006) to study military mental health professionals. Bride and Kintzle (2011) explored STS, job satisfaction, and propensity toward job turnover by utilizing both the STSS (to analyze secondary stress) and the Professional Quality of Life Scale (ProQOL; Stamm, 2005). Cieslak et al. (2014) evaluated 41 studies in a meta-analysis; the STSS was utilized in 6 of the 41 instances, paired with another measure, to study stress and burnout. The minimal use of the STSS for these studies (14.6\%) speaks to the idea that the researchers were interested in studying exhaustion, cynicism, and a lack of accomplishments, which are not highlighted components of the DSM-IV PTSD criteria. Bride et al. (2007) encouraged researchers and clinicians to select their instruments carefully, matching the goals of the study or intervention to the aspects most important for measuring. Because the current study explored information that teachers felt was related to topics that include PTSD themes of intrusion, avoidance, and arousal, the STSS is a good fit for these goals (Bride et al., 2007).

Secondary Traumatic Stress Scale. The Secondary Traumatic Stress Scale (STSS: Bride et al., 2004) is a self-report survey instrument that was designed to measure STS symptoms, especially within the three PTSD subscales of intrusion, avoidance, and arousal. The 17-item instrument will be described, the development of the measure will be explored, and the 
mechanism of STS will be explained, providing background information for the analysis of the themes that result from the use of the instrument.

Description of the STSS. The STSS is a 17-item assessment instrument (Appendix A), developed in response to a lack of instrumentation to measure secondary trauma in providers of supportive services for trauma-survivor clientele. Although the psychological impact of direct trauma has been studied, documented, and assessed, the implications for service providers (secondary effects) have also been noted and are also considerable (Chrestman, 1999; Figley, 1999).

History of the STSS development. In its initial stages, Figley (1999) developed the framework for the scale from the definition of PTSD, derived from the Diagnostic and Statistical Manual of Mental Disorders (DSM-IV; American Psychiatric Association, 1994). The scale included the subscales of intrusion, avoidance, and arousal. The original Likert-survey of 65 items, probing these three areas, was reviewed by trauma experts. A small pilot test with 37 direct service providers reduced the instrument to 50 items. Two hundred respondents completed the second stage test of the STSS instrument, which, after fine-tuning with factor analysis, reduced the scale to 17 items. These 17 items represent one probe for each of the 17 individual symptoms listed in the DSM-IV criteria, which are related to the PTSD diagnostic features in the three subscales (intrusion, avoidance, and arousal). The STSS is currently used widely in the United States (Molnar et al., 2017) and internationally (Jacobs, Charmillot, Martin Soelch, \& Horsch, 2019; Setti \& Argentero, 2012; Turliuc, Măirean, \& Turliuc, 2015; Yildirim, Kidak, \& Yurdabakan, 2018). The validity of the instrument is discussed in detail in the methodology section. 
Development of the diagnostic features. Because STS is a construct that is similar to PTSD, the STSS was developed using the PTSD diagnostic framework from DSM-IV. However, the DSM-IV has iterated through several revisions since the development of the STSS. A comparison of the DSM-IV and DSM-V constructs can aid in the understanding of the STSS.

The STSS is based on the DSM-IV criteria of PTSD symptoms. As the field of traumatology has developed, the diagnostic elements of PTSD have also developed. Because of this change, the research on STS, along with the STSS, has evolved. Based on the PTSD nomenclature embodied in the 17 symptoms listed in the DSM-IV, the STSS was developed with a three-factor subscale of arousal, intrusion, and avoidance (Bride et al., 2004). Mordeno et al. (2017) noted that the PTSD nomenclature in the DSM-V is based on 20 symptoms and organizes around "intrusion, avoidance, hyperarousal, and negative alterations in cognitions and mood (NACM)" (p. 155). Recognizing this difference between DSM-IV and DSM-V, Mordeno et al. (2017) noted that there is no update to the STSS to take into account the developments in the DSM-V. Whereas there is not a revised STSS currently, there are several models of STS that take the DSM-V into account, as shown in Table 2-4.

Table 2-4: DSM-V Inspired Traumatic Stress Models

\begin{tabular}{|c|c|c|}
\hline Model & Researchers & Model Characteristics \\
\hline $\begin{array}{c}\text { Model 1: Avoidance and } \\
\text { numbing separation }\end{array}$ & $\begin{array}{c}\text { King et al. (1998) } \\
\text { Wang et al. (2015) }\end{array}$ & $\begin{array}{c}\text { Avoidance separated into avoiding and } \\
\text { numbing }\end{array}$ \\
\hline $\begin{array}{c}\text { Model 2: General distress } \\
\text { and dysphoria }\end{array}$ & $\begin{array}{c}\text { Watson (2005) } \\
\text { Watson (2009) }\end{array}$ & $\begin{array}{c}\text { Identify common and unique symptoms } \\
\text { of PTSD (because distress and } \\
\text { dysphoria are common to other } \\
\text { disorders) }\end{array}$ \\
\hline Model 3: Dysphoric Arousal & $\begin{array}{c}\text { Elhai et al. (2011) } \\
\text { Armour et al. } \\
(2013)\end{array}$ & $\begin{array}{c}\text { Bridges between model 1 and model 2; } \\
\text { separate symptoms into discrete clusters }\end{array}$ \\
\hline
\end{tabular}




\begin{tabular}{|c|c|c|}
\hline $\begin{array}{c}\text { Model 4: Anhedonia (with } \\
\text { two constructs) }\end{array}$ & $\begin{array}{c}\text { Liu et al. (2014) } \\
\text { Armour et al. } \\
(2015)\end{array}$ & $\begin{array}{c}\text { Separates anhedonia into negative affect } \\
\text { and reduced positive affect }\end{array}$ \\
\hline $\begin{array}{c}\text { Model 5: Externalizing } \\
\text { Behaviors }\end{array}$ & $\begin{array}{c}\text { Armour et al. } \\
(2015)\end{array}$ & $\begin{array}{c}\text { Similar to dysphoric arousal but adds } \\
\text { aggressive and destructive-like } \\
\text { behaviors }\end{array}$ \\
\hline $\begin{array}{c}\text { Model 6: Seven-Factor } \\
\text { Model }\end{array}$ & $\begin{array}{c}\text { Amour et al. } \\
(2015)\end{array}$ & $\begin{array}{c}\text { Seven factors are the following: } \\
\text { Re-experiencing }\end{array}$ \\
& $\begin{array}{c}\text { Mordeno et al. } \\
(2016)\end{array}$ & $\begin{array}{c}\text { Avoidance } \\
\text { Anhedonive affect }\end{array}$ \\
& & Externalizing behaviors \\
& & Anxious symptom clusters \\
& & Dysphoric symptom clusters \\
\hline
\end{tabular}

Note. Table adapted from Mordeno et al. (2017).

It might be possible to use one of these models, in the future, to revise the STSS. For this teacher study, the 17 items of the STSS based on DSM-IV (Bride et al., 2004) are sufficiently rigorous for the expected results, primarily based on the STS themes for educators.

Theory and mechanism of STS. The definition, diagnostic framework, and measurement of STS coalesce around a theory of secondary trauma and the constellation of responses that result from working with people who have experienced trauma. Ludick and Figley (2017) proposed a theory of STS with nine working mechanisms: (1) STS is complex and can occur from working with people who have been traumatized, by studying them, or by reading about them, and it is often unavoidable; (2) the amount of contact of exposure varies depending on the person, and it can be first-person contact, videos, written records, or even photos; (3) empathy elevates STS as the worker assists or helps the person who has been traumatized; (4) compartmentalizing the stress reaction to the contact (like with pictures, videos, etc.) also elevates the STS; (5) prolonged exposure raises STS; (6) remembering prior traumatic events 
elevates STS; (7) STS is lowered when compassion stress satisfaction is experienced by the worker in a way that increases a sense of worth and purpose; (8) when social support is experienced by the worker from peers and the workplace, STS is lowered; and (9) compassion fatigue resilience (CFR) directly affects STS, as does life demands outside of work. Ludick and Figley noted that compassion stress satisfaction is pleasure or satisfaction from doing work well, particularly the work of helping (Stamm, 2005), and compassion fatigue resilience is compassion from empathy that is protective. Both are related to STS in that as both increase, STS decreases.

\subsubsection{Secondary Traumatic Stress in Helping Professions}

Ludick and Figley (2017) clarified that while compassion fatigue is a term that is used more often with helping professions, STS is used with a more diverse population. In fact, in the research literature, STS has been noted in a wide diversity of jobs including mental health (Catherall, 1999; Hensel, Ruiz, Finney, \& Dewa, 2015; Kassam-Adams, 1999; McCann \& Pearlman, 1990), psychology (Chrestman, 1999), psychiatry (Bills, 1999), and social work (BenPorat \& Itzhaky, 2011; Bride et al., 2004; Tosone, McTighe, Bauwens, \& Naturale, 2011). STS research also includes the medical fields like doctors and nurses in hospital settings (Granek et al., 2017; Markwell \& Wainer, 2009; Morrison \& Joy, 2016; Nimmo \& Huggard, 2013; Townsend \& Campbell, 2009), disaster response (McLennan, Evans, Cowlishaw, Pamment, \& Wright, 2016), and even 9-1-1 emergency response (Pierce \& Lilly, 2012). STS research now includes refugee resettlement (Akinsulure-Smith, Espinosa, Chu, \& Hallock, 2018), firefighting (Lee, Lee, Kim, Jeon, \& Sim, 2017), ancillary veteran work (Bachem et al., 2018; Yager, Gerszberg, \& Dohrenwend, 2016), religious work (Flannelly, Roberts, \& Weaver, 2005), and even journalism (Browne, Evangeli, \& Greenberg, 2012). Ludick and Figley (2017) noted that this diversity reflects the "wide-angled research focus, to include anyone reading or thinking 
about traumatic materials" (p. 112). This section first addresses the three scoring methods of the STSS, which informs the subsequent discussion of the prevalence of STS as determined by the STSS (Bride et al., 2004). Then the section will conclude with an examination of the risk factors, the protective factors, and the interventions for STS.

Three interpretations of STS scores on the STSS. Using the STSS, there are at least three different ways to assess for STS (Bride, 2007; Bride et al., 2007). These are percentile-based interpretation, algorithm-based interpretation, and cutoff interpretation.

Percentile-based interpretation. Percentile-based interpretation of test scores is made by comparing an individual or group score to normative scores, which are based on percentile. On the full STSS, the scores range from 17-85. Bride (2007) offers this percentile interpretation for the scoring of the STSS: Scores less than 28 points are below the 50th percentile: no STS. Scores between 28 - 37 points are between 51 st to the 75 th percentile: mild STS. Scores between $38-$ 43 points are between the 76 th to the 90 th percentile: moderate STS. Scores between $44-48$ points are between the 91st to the 95th percentile: high STS. Scores at 49 points or above are at or above the 95th percentile: severe STS. With percentile-based scoring, the whole test or full test score of the STSS is often reported, but not always (McDonald \& Calhoun, 2010). Table 2-5 shows an example of whole-test reporting, where the mean and standard deviation of the whole population are compared. Full test scoring offers standardized comparisons of large populations, but the subscale details are not delineated when reporting a whole-scale or full-scale score.

Some researchers report a whole-test score; others report subscales or mixed subscales as a symptom cluster method (McDonald \& Calhoun, 2010). Table 2-6 shows the range and percentile for the STSS subscales, compared to the full test. To see the application of this 
percentile method with symptom clusters, Table 2-7 reports the comparison of the mean and standard deviation comparison for STSS subscales.

Algorithm-based interpretation. The algorithm approach to interpreting the STSS score is linked to the PTSD diagnostic framework. Scoring for this method is as follows: to meet the criteria for an individual core subscale, an individual must endorse at least one item on the intrusion subscale, or at least three items on the avoidance subscale, or at least two items on the arousal subscale. Table 2-8 shows respondents who meet the criteria for one subscale. Bride (2007) noted that a symptom is considered present if the corresponding STSS item is rated three or higher (that is, occasionally, often, or very often). All three of the above core subscale criteria must be met simultaneously to meet the diagnostic criteria for PTSD. An individual must endorse at least one item on the intrusion subscale, and at least three items on the avoidance subscale, and at least two items on the arousal subscale. As with individual subscale endorsements, Bride (2007) noted that a symptom is considered present if the corresponding STSS item is rated 3, 4, or 5 (that is, occasionally, often, or very often). The prevalence of fullPTSD criteria, as scored by the algorithm method, can be seen in Table 2-9, which shows the comparison of frequency for full endorsement of PTSD.

It should be noted that the full endorsement of PTSD on the STSS means that respondents might meet the diagnosis of PTSD if a clinician were to administer a standardized assessment for PTSD. It is not to be interpreted as a clinical diagnosis of PTSD. PTSD is a diagnosis that can only be made by a mental health professional; a self-check instrument cannot determine it.

Cutoff reporting. Some researchers report a whole-test score; others report subscales or mixed subscales as a symptom cluster method (McDonald \& Calhoun, 2010). Other researchers 
reported cut-off scores. Bride (2007) recommended a cut-off score of 38, which is the lower end of the moderate range, as the cutoff for consideration of PTSD due to STS. Bride noted the following:

That is, using a cutoff value of 38,93 percent of those who met the core criteria for PTSD using the algorithm approach would be correctly identified as having PTSD and 91 percent of those who did not meet the core criteria for PTSD would be identified as not having PTSD. (p. 68)

While I did not identify studies interpreting the STSS using cut-off interpretation, I did include this as part of my data collection and analysis. Again, the same caveat regarding PTSD pertains to cutoff scoring: the STSS offers an indication of consideration of PTSD symptoms. The cutoff at 38 means respondents might meet the diagnosis of PTSD if a clinician were to administer a standardized assessment for PTSD.

Prevalence of STS. Because STS is a construct that impacts professionals and helpers who are exposed to the traumatic experiences of others, at this time, there is not a national number or prevalence for exposure for all professionals. There are, however, various studies that report the STS level for different occupations (see Table 2-5). Using percentile-based interpretation, it is possible to cross-compare occupation STS levels.

Table 2-5: Comparison of Mean and Standard Deviation for Full STSS Score

\begin{tabular}{|l|c|c|}
\hline \multicolumn{1}{|c|}{ Study } & $\begin{array}{c}\text { Sample size/ } \\
\text { Population }\end{array}$ & Full STSS Score $^{\mathrm{a}}$ \\
\hline Ting et al. (2005) & $\begin{array}{c}\mathrm{N}=275 \\
\text { Social workers }\end{array}$ & $33.30^{\mathrm{b}}$ \\
\hline $\begin{array}{l}\text { Perron, B., \& Hiltz, B. } \\
(2006)\end{array}$ & $\begin{array}{c}\mathrm{N}=66 \\
\text { Forensic interviewers of abused } \\
\text { children }\end{array}$ & $34.2(10.6)$ \\
\hline
\end{tabular}




\begin{tabular}{|c|c|c|}
\hline Bride (2007) & $\begin{array}{c}\mathrm{N}=282 \\
\text { Social workers }\end{array}$ & $29.69(10.74)$ \\
\hline $\begin{array}{l}\text { Bride et al. (2009); } \\
\text { Bride \& Kintzle (2011) }\end{array}$ & $\begin{array}{c}\mathrm{N}=225 \\
\text { Substance Abuse Counselors }\end{array}$ & $31.20(12.30)$ \\
\hline $\begin{array}{l}\text { Ben-Porat, A., \& } \\
\text { Itzhaky, H. (2011) }\end{array}$ & $\begin{array}{c}\mathrm{N}=143 \\
\text { Domestic Violence Therapists } \\
\mathrm{n}=103 \\
\text { Received training } \\
\mathrm{n}=40 \\
\text { Did not receive training }\end{array}$ & $\begin{array}{l}38.42(6.80) \\
41.48(9.18)\end{array}$ \\
\hline $\begin{array}{l}\text { Smith Hatcher et al. } \\
\text { (2011) }\end{array}$ & $\begin{array}{c}\text { N=118 } \\
\text { Juvenile justice teachers and staff }\end{array}$ & $37.74(10.74)$ \\
\hline Beckmann (2015) & $\begin{array}{c}\mathrm{N}=92 \\
\text { Disaster Mental Health Volunteers } \\
\text { (Red Cross) }\end{array}$ & $\begin{array}{l}28.34(9.36) \\
\qquad(\mathrm{n}=81) \\
\text { However, } 11.63 \% \\
\text { respondents }(\mathrm{n}=10) \mathrm{had} \\
\text { scores above the cut-off } \\
\text { score of } 38\end{array}$ \\
\hline McLennan et al. (2016) & $\begin{array}{c}\mathrm{N}=33 \\
\text { Post-disaster field research interviewers }\end{array}$ & $26.42(8.00)$ \\
\hline Morrison \& Joy (2016) & $\begin{array}{c}\mathrm{N}=80 \\
\text { Emergency Nurses (Scotland) }\end{array}$ & $37.40(17.0)$ \\
\hline Mordeno et al. (2017) & $\begin{array}{l}\mathrm{N}=241 \\
\text { Nurses (Philippines) }\end{array}$ & $30.38(10.72)$ \\
\hline $\begin{array}{l}\text { Akinsulure-Smith et al. } \\
\text { (2018) }\end{array}$ & $\begin{array}{l}\mathrm{N}=210 \\
\text { Refugee Resettlement Workers }\end{array}$ & $36.67(13.36)$ \\
\hline Denham (2018) & $\begin{array}{l}\qquad \begin{array}{l}N=172 \\
\text { High school teachers, no school decay } \\
(n=84)\end{array} \\
\text { High school teachers, school decay } \\
(n=88)\end{array}$ & $\begin{array}{l}20.80(13.377) \\
34.03(13.977)\end{array}$ \\
\hline
\end{tabular}

${ }^{a}$ Scores less than 28 points are below the 50th percentile: no STS. Scores between $28-37$ points are between 51st to the 75th percentile: mild STS. Scores between $38-43$ points are between the 76th to the 90th percentile: moderate STS. Scores between $44-48$ points are between the 91 st to the 95 th percentile: high STS. Scores at 49 
points or above are at or above the 95 th percentile: severe STS. ${ }^{\text {b }} S D$ reported by each question instead of by total; $S D$ ranged from $.81-1.11$

The construct of STS, as assessed by the STSS, is seen in almost every sample population at the mild or moderate level. From Table 2-5, 11 of 12 studies show participants at the mild to moderate range of STS (91.22\% of studies). The only study that shows no STS is with interviewers of people who went through a natural disaster (McLennan et al., 2016). The interviewers did not interact with clients to offer any services; they listened to the narratives of people who had been through fires. It is interesting to note that the participants who interacted with current trauma clients or students appeared to have higher scores. For example, disaster volunteers (Beckmann, 2015) and post-disaster interviewers (McLennan et al., 2016) had the lowest full STS scores.

In contrast, juvenile justice teachers (Smith Hatcher, Bride, Oh, Moultrie King, \& Franklin Catrett, 2011), working with juvenile offenders, have higher levels of STS. Juvenile offenders have a higher rate of trauma than non-offending youth (Abram et al., 2004; Smith Hatcher et al., 2011), which could account for the higher stress for the participants $(\mathrm{N}=118)$ in Smith Hatcher et al. (2011), especially in comparison to McLennan et al. (2016) or Beckmann (2015). However, even with the low full score for Beckmann (2015), 11.63\% of respondents scored at the moderate range. Reporting full scores for the whole population can mask these results, at times. The standard deviation for many of the full scores means that, like Beckmann (2015), there will be a percentage of respondents who have higher and lower scores that might not be readily apparent to the reader.

The full STSS score is not the only score that is reported on secondary stress. The three subscale scores are also detailed in several ways. Because STS is related to PTSD, Bride et al. (2004) developed the STSS based on the diagnostic framework of PTSD, primarily focused on 
the three subscales of intrusion, avoidance, and arousal. For reports of the subscale findings, see Tables $7-9$. To help with the interpretation of these results, Bride (2007) offered an interpretation scale (see Table 2-6).

Table 2-6: Range and Percentile for the STSS Subscales and Full Test

\begin{tabular}{|c|c|c|c|c|c|c|c|}
\hline \multicolumn{8}{|c|}{ Percentile } \\
\hline & Range & 25th & 50th & 75th & 90th & 95th & $\begin{array}{c}\text { Above } \\
\text { 95th }\end{array}$ \\
\hline & & No STS & No STS & $\begin{array}{c}\text { Mild } \\
\text { STS }\end{array}$ & $\begin{array}{c}\text { Moderate } \\
\text { STS }\end{array}$ & $\begin{array}{c}\text { High } \\
\text { STS }\end{array}$ & $\begin{array}{c}\text { Severe } \\
\text { STS }\end{array}$ \\
\hline $\begin{array}{l}\text { Intrusion } \\
\text { Subscale }\end{array}$ & $5-25$ & 6.00 & 7.00 & 11.00 & 12.00 & 13.00 & $>13.00$ \\
\hline $\begin{array}{c}\text { Avoidance } \\
\text { Subscale }\end{array}$ & $7-35$ & 8.00 & 12.00 & 16.00 & 20.00 & 22.00 & $>22.00$ \\
\hline $\begin{array}{c}\text { Arousal } \\
\text { Subscale }\end{array}$ & $5-25$ & 6.00 & 8.00 & 11.00 & 14.00 & 16.00 & $>16.00$ \\
\hline Full STSS & $17-85$ & 21.00 & 27.00 & 37.00 & 43.80 & 48.40 & $>48.40$ \\
\hline
\end{tabular}

Note. Adapted from Bride (2007).

Again it is noteworthy to see the higher mean and standard deviation for respondents who work with students of high trauma (Smith Hatcher et al., 2011). The higher mean can be seen with detail by looking at the subscales in Table 2-7.

Table 2-7: Comparison of Mean and Standard Deviation Comparison for STSS Subscales

\begin{tabular}{|c|c|c|c|}
\hline Study & $\begin{array}{c}\text { Sample size/ } \\
\text { Population }\end{array}$ & $\begin{array}{c}\text { Individual } \\
\text { Subscale }\end{array}$ & $\begin{array}{c}\text { Individual Subscale } \\
\text { Scores }^{\mathbf{a}}\end{array}$ \\
\cline { 3 - 4 } & & & $\boldsymbol{M}(\boldsymbol{S D})$ \\
\hline Ting et al. & $\mathrm{N}=275$ & Intrusion & $9.91^{\mathrm{b}}$ \\
$(2005)$ & Avoidance & 13.21 \\
& Social workers & Arousal & 10.18 \\
\hline Bride (2007) & $\mathrm{N}=282$ & Intrusion & $8.18(3.04)$ \\
& & Avoidance & $12.58(5.00)$ \\
& Social workers & Arousal & $8.93(3.56)$ \\
\hline Smith Hatcher & $\mathrm{N}=118$ & Intrusion & $10.64(3.19)$ \\
et al. (2011) & & Avoidance & $15.73(4.90)$ \\
\hline
\end{tabular}




\begin{tabular}{|c|c|c|c|}
\hline & $\begin{array}{c}\text { Juvenile justice teachers and } \\
\text { staff }\end{array}$ & Arousal & $11.37(3.79)$ \\
\hline Beckmann & $\mathrm{N}=92$ & Intrusion & $8.08(3.12)$ \\
$(2015)$ & Disaster Mental Health & Avoidance & $11.06(3.95)$ \\
& Volunteers (Red Cross) & Arousal & $8.36(3.24)$ \\
\hline Morrison \& & $\mathrm{N}=80$ & Intrusion & $10.6(4.8)$ \\
Joy (2016) & & Avoidance & $15.3(7.5)$ \\
& Emergency Nurses & Arousal & $11.6(5.4)$ \\
& (Scotland) & & \\
\hline
\end{tabular}

a Please see Table 2-6 for the interpretation of these scores. ${ }^{\mathrm{b}} S D$ reported by each question instead of by total; $S D$ ranged from .81-1.11.

In Table 2-8, the individual subscales are reported by frequency. This method is more granular because it counts not by aggregated group, but by individuals who endorse subscale criteria within the PTSD subscale groupings.

Table 2-8: Comparison of Frequency of STSS Respondents Who Meet Criteria for One Subscale

\begin{tabular}{|c|c|c|c|}
\hline \multirow[t]{2}{*}{ Study } & \multirow[t]{2}{*}{$\begin{array}{l}\text { Sample size/ } \\
\text { Population }\end{array}$} & \multicolumn{2}{|c|}{$\begin{array}{c}\text { Criteria for one of the core } \\
\text { subscale symptom clusters met }\end{array}$} \\
\hline & & \multicolumn{2}{|c|}{$(\%)$} \\
\hline \multirow[t]{3}{*}{ Bride (2007) } & $\mathrm{N}=282$ & Intrusion & $128(45.0)$ \\
\hline & & Avoidance & $71(25.2)$ \\
\hline & Social workers & Arousal & $71(25.2)$ \\
\hline \multirow{3}{*}{$\begin{array}{l}\text { Bride et al., (2009); } \\
\text { Bride \& Kintzle } \\
(2011)\end{array}$} & $\mathrm{N}=225$ & Intrusion & $104(48.1)$ \\
\hline & & Avoidance & $59(27.3)$ \\
\hline & Substance Abuse Counselors & Arousal & $59(27.3)$ \\
\hline \multirow{3}{*}{$\begin{array}{l}\text { Smith Hatcher et al. } \\
\text { (2011) }\end{array}$} & $\mathrm{N}=118$ & Intrusion & $90(76.3)$ \\
\hline & & Avoidance & $59(50)$ \\
\hline & $\begin{array}{l}\text { Juvenile justice teachers and } \\
\text { staff }\end{array}$ & Arousal & $58(49.2)$ \\
\hline \multirow{3}{*}{$\begin{array}{l}\text { Morrison \& Joy } \\
\text { (2016) }\end{array}$} & $\mathrm{N}=80$ & Intrusion & $57(71)$ \\
\hline & & Avoidance & $33(41)$ \\
\hline & Emergency Nurses (Scotland) & Arousal & $43(54)$ \\
\hline
\end{tabular}

${ }^{a}$ This table represents score percentages for individual subscales. Scoring for this method is as follows: To meet the criteria for a core subscale, an individual must endorse at least one item on the intrusion subscale, or at least three 
items on the avoidance subscale, or at least two items on the arousal subscale. Bride (2007) noted that a symptom is considered present if the corresponding STSS item is rated 3, 4, or 5 (that is, occasionally, often, or very often).

Finally, this last table (Table 2-9) shows a comparison of studies, by frequency of respondents who meet the criteria for PTSD, by affirming a total of questions in all of the subscales. An example of the algorithm-interpretation method, all three of the subscales must be endorsed to be counted.

Table 2-9: Comparison of Frequency of Subscale Diagnostic Criteria of PTSD

\begin{tabular}{|l|c|c|}
\hline \multicolumn{1}{|c|}{ Study } & $\begin{array}{r}\text { Sample size/ } \\
\text { Population }\end{array}$ & $\begin{array}{c}\text { PTSD Subscale } \\
\text { Endorsement } \text { Met }^{\mathbf{b}}\end{array}$ \\
\cline { 2 - 3 } & $\begin{array}{c}\mathrm{N}=282 \\
\text { Social workers }\end{array}$ & $43(15.2)$ \\
\hline $\begin{array}{l}\text { Bride et al., (2009); Bride } \\
\text { \& Kintzle (2011) }\end{array}$ & $\begin{array}{c}\mathrm{N}=225 \\
\text { Substance Abuse Counselors }\end{array}$ & $41(19)$ \\
\hline Smith Hatcher et al. (2011) & $\begin{array}{c}\mathrm{N}=118 \\
\text { Juvenile justice teachers and staff }\end{array}$ & $46(39)$ \\
\hline Morrison \& Joy (2016) & $\begin{array}{c}\mathrm{N}=80 \\
\text { Emergency Nurses (Scotland) }\end{array}$ & $31(39)$ \\
\hline
\end{tabular}

${ }^{\mathrm{b}}$ To meet this requirement, a respondent must endorse at least one item on the intrusion subscale, plus at least three items on the avoidance subscale, plus at least two items on the arousal subscale (Bride et al., 2009). This means all three subscale endorsements must be met for the criteria of PTSD to be met.

Many helping professionals who have been assessed for STS show a mild or moderate level of STS. As shown in Table 2-9, approximately 15-39\% also meet the criteria for PTSD, as assessed by the STSS (Bride et al., 2004). The STSS PTSD prevalence is higher than the prevalence of PTSD assessed in other ways. Berger et al. (2011), from meta-regression analysis of 28 studies with 40 samples $(\mathrm{N}=20,424)$, reported that the global PTSD prevalence was around $10 \%$ (95\% CI: 8.1-11.9\%) for emergency workers. When the variables were stratified, they revealed different prevalence estimates (Berger et al., 2011, p. 6): "requirement of impairment for the diagnosis of PTSD" $(\chi 2=18.31 ; d f=2 ; P<0.001)$, "geographic location" 
$(\chi 2=13.39 ; d f=5 ; P=0.02)$, "occupational group" $(\chi 2=16.37 ; d f=3 ; P=0.001)$, and "type of work" $(\chi 2=5.93 ; d f=2 ; P=0.05)$. The STSS-related PTSD prevalence is higher than this global report of PTSD, possibly due to the self-reported nature of the STSS and that STS is a construct that is merely one part of PTSD.

\subsection{STS Risk Factors, Preventative Factors, and Interventions}

Certain factors have been studied to ascertain if they place people at higher risk or, conversely, if they offer a protective factor when it comes to STS. Specific interventions have been studied to see if they impact the level of STS in helpers.

\subsubsection{Risk Factors}

In the STS literature, several risk factors coalesce from studies of STS. In a study of social workers ( $\mathrm{N}=287)$, Bride et al. (2004) found that risk factors for STS were correlated with the percentage of traumatized clients on a caseload $(M=3.19, S D=.87, r=.260)$ and the time working on the trauma issues when working with these clients $(M=3.49, S D=.93, r=.232)$. Hensel et al. (2015) found similar weak correlations in meta-analysis ( $\mathrm{N}=38$ studies), with the following correlations for risk factors for STS: trauma caseload volume $(r=.16)$, caseload frequency $(r=.12)$, caseload ratio $(r=.19)$, and having personal trauma history $(r=.19)$.

Narratively, Bride and Figley (2009) noted that younger helping professionals might be more at risk. Professionals with less experience "providing trauma services" (p. 320) are at greater risk. Higher exposure to trauma-related topics and activities elevates the risk (Brady, Guy, Poelstra, \& Brokaw, 1999). However, the length of time was found to be less important compared to the level of trauma. Professionals who work longer with less severe clients seem to be less impacted (Chrestman, 1999; Kassam-Adams, 1999). The caseload ratio also has a bearing on risk; if helpers have clients with more severe trauma but can balance that with nontherapeutic 
work or nonvictim clients, the risk can decrease (Hensel et al., 2015). According to researchers, the helper who has a history of trauma, especially childhood trauma, is at a higher risk level (Akinsulure-Smith et al., 2018; Kassam-Adams, 1999; Pearlman \& Mac Ian, 1995).

Demographic factors. Some factors do appear to be related to elevated risk for secondary stress. Gil and Weinberg (2015) noted associations in a sample of therapists $(\mathrm{N}=160)$ through independent t-tests for the following demographics: female participants $(M=0.68 ; S D=0.56 ; t=$ 2.4; $p<.01)$ reporting higher stress than males $(M=0.30 ; S D=0.42)$, and respondents with a trauma history $(M=0.81 ; S D=0.36)$ reporting higher levels than respondents with no trauma history reported $(M=0.41 ; S D=0.44)$. Rojas-Flores et al. (2015) also confirmed this finding, with teachers with a trauma history showing higher levels of PTSD symptomatology than those without a trauma history (Study 1 with $\mathrm{N}=193$ : 95\% CI $=.04-.16, z=3.31, p=.0009$; Study 2 with $\mathrm{N}=257: 95 \% \mathrm{CI}=.06-.31, z=3.19, p=.001)$.

Gil and Weinberg (2015) used ANOVA to determine that respondents who reported a lack of supervision $(M=0.71 ; S D=0.56)$ had higher secondary trauma symptoms than those who reported steady $(M=0.39 ; S D=0.22)$ or irregular $(M=0.45 ; S D=0.24)$ forms of supervision. Beckmann (2015) found weak positive correlations between the STSS total score and the following demographic variables: being a young adult $(0.258, p<.01)$; being single (for arousal subscale only, $0.307, p<.01)$; and sometimes engaging in self-care $(0.430, p<.001)$. This last point about self-care was contrasted with the idea of always engaging in self-care, which was negatively correlated $(-0.476, p<.001)$. So sometimes engaging in self-care increases the risk for STS; however, always engaging in self-care has more of a protective function.

Protective factors. The research with STS is still developing; however, there are some indicators that there might be some factors that work as protective factors for secondary stress. 
McLennan et al. (2016) noted that over $60 \%$ of respondents $(\mathrm{N}=33)$, who were post-disaster interviewers, attested to the positive impact of their work and that the work would support future risk reduction. Beckmann (2015) found, through multiple regression analysis, that certain protective factors decreased the risk of secondary traumatic stress in disaster relief workers $(\mathrm{N}=$ 92). This decrease was not seen in the overall, total STSS score but rather in the subscale score. These factors for the intrusion subscale included the following: working with trauma survivors outside of their volunteer work as associated with less intrusion $(B=-1.732)$ and $7-12$ months since last disaster $(B=1.783)$ associated with more intrusion than the previous disaster over 13 months ago. In other words, intrusive symptoms benefit from working with additional trauma survivors outside of disasters and having more time in between disaster responses. On the arousal subscale, those who were unemployed had significantly less arousal symptomology $(B=$ -4.376) than those who were employed. It should be noted that in STS literature, protective factors are sometimes referred to as posttraumatic growth (Beckmann, 2015).

Interventions for STS. Bercier and Maynard (2015), in their systematic review for trauma intervention programs, reviewed 159 full-text reports, finding none rigorous enough concerning study design, participant characteristics, and coding. Notably, the researchers started with a pool of 4,134 titles. Sprang et al. (2019) supported this same idea by noting, "the lack of a strong evidence base to inform STS assessment and intervention" (p. 74). Sprang et al. list the following, although with the caveat that there is not an evidence-base for these interventions: strategy-based clinical descriptions, self-help programs, structured workshops, in-person and online training, and wellness promotions.

Bober and Regehr (2006) described the belief in the usefulness of self-care for intervention. In their study $(\mathrm{N}=259)$ with therapists, though, the belief in self-care did not 
translate into time in self-care nor a decreased level of STS. Although therapists affirmed selfcare mean scores at time with family $3.36(\mathrm{SD}=0.75)$, vacation $3.55(\mathrm{SD}=0.71)$, movies/TV $2.62(\mathrm{SD}=1.01)$, hobbies $3.16(\mathrm{SD}=0.80)$, and exercise $3.34(\mathrm{SD}=0.83)$, correlations with trauma scores were not associated, with $r$-values reported at -0.03 for the self-care category. So although the therapists felt self-care was important, self-care was not an intervention that was correlated with lower STS.

Berger, Abu-Raiya, and Benatov (2016) evaluated a school-based intervention by comparing educators in a control group $(n=26)$ with those in an intervention group $(n=37)$ to determine whether the intervention reduced both posttraumatic distress and STS symptoms. While the research was conducted within the context of emergency response, the results are significant concerning STS, as measured by the Professional Quality of Life scale (ProQOL; Stamm, 2010): Time X Scale X Group; Wilk’s Lambda $=.39 ; F=22.82 ; d f=4,58 ; p<.001$. When comparing the control group to the intervention group, the intervention group significantly reduced their posttraumatic stress and STS symptoms.

Finally, the National Child Trauma Stress Network (NCTSN), while noting the limited evidentiary basis of intervention programs, described cognitive-behavioral and mindfulness practices as "emerging as best practices" (National Child Traumatic Stress Network, Secondary Traumatic Stress Committee, 2011, sec. 5). There is some support that interventions like these might be more beneficial to helpers: Gil and Weinberg (2015), using a Pearson correlation analysis $(\mathrm{N}=160)$, found a positive correlation $(\mathrm{r}=.28 ; \mathrm{p}<.001)$ between the use of emotionfocused coping strategies (such as emotional ventilation) and the level of secondary trauma symptoms and a positive correlation $(\mathrm{r}=.22 ; \mathrm{p}<.001)$ between avoidance-coping strategies (such as behavioral disengagement and alcohol/drug use) and the level of secondary trauma symptoms. 
These represent coping mechanisms for STS that might not be as useful or beneficial for helpers. Determining the best practices, by winnowing out those interventions which raise STS, could be a productive research-based start for developing an intervention or support framework.

\subsection{Secondary Traumatic Stress Experienced by Educators}

Studies for educators with STS are limited compared to the amount of research about teacher stress and burnout; most of the STS research is focused on disaster response or is located internationally (Denham, 2018; Rojas-Flores et al., 2015). Although educators are not typically considered so-called "Professionals who work therapeutically with victims of trauma" (Hensel et al., 2015), there are some researchers who have utilized this lens and studied teachers with traumatic stress. Findings from these studies open the more comprehensive research conversation for educators with STS (see Table 2-10). In this section, information from the limited number of studies about teacher STS will be examined. First, the prevalence of general stress experienced by educators will be discussed. The prevalence will provide background for an exploration of studies of secondary stress experienced by educators, as well as the themes that coalesce from those studies.

\subsubsection{Prevalence of Stress Experienced by Educators}

Teaching is considered a "high stress" profession (Herman, Hickmon-Rosa, \& Reinke, 2017). Precarity in the teaching profession is underscored by data tracked by the U.S. Bureau of Labor Statistics (Sauter, 2017). Incidence rates in 2014 for occupational injuries and illnesses (U.S. Department of Labor, 2015) of 4.2 cases per 100 for public elementary and secondary teachers exceeded service jobs (3.0), construction industries (3.6), and even manufacturing sectors (4.0). These injuries and illnesses include stress-related occupational factors (Kyriacou, 2001; McIntyre, McIntyre, \& Francis, 2017; Montgomery \& Rupp, 2005). Teacher 
dissatisfaction and stress can lead to burnout (Kyriacou, 2001), which, in turn, can drive teacher turnover (Sutcher, Darling-Hammond, \& Carver-Thomas, 2019). Podolsky, Kini, Bishop, and Darling-Hammond (2016) highlighted the fact that $8 \%$ of all public-school teachers left the profession after the 2012 school year $(\mathrm{N}=270,200)$. Carver-Thomas and Darling-Hammond (2017) noted that, of the approximately $90 \%$ of open teacher positions that are created by these teachers who leave the profession, one-third leave due to retirement and about two-thirds leave due to dissatisfaction. Regardless of the reasons, students are impacted, because "Teachers are the number one in-school influence on student achievement" (Carver-Thomas and DarlingHammond, 2017, para. 2). These attrition rates are higher in high-poverty schools (as defined by free and reduced-price lunch programs). In the $2012-13$ school year, schools with $75 \%$ or more of students receiving free or reduced-price lunches had a teacher attrition rate of almost one in 10 teachers, which contrasted with one in 15 teachers for schools with $34 \%$ or fewer students receiving free or reduced-price lunches (Goldring, Taie, \& Riddles, 2014; Podolsky et al., 2016). While there are no national statistics for teachers that reflect secondary stress, these statistics indicate that teachers who leave the profession may do so at different rates depending on environmental stressors.

As an additional note, because teacher STS is a construct predicated on a mechanism that relies on exposure to trauma through students, it is also possible to examine the literature of "trauma-informed" schools, policies, and research to glean information about secondary stress for teachers. For example, Atallah, Koslouski, Perkins, Marsico, and Porche, (2019) found that educators and educational stakeholders $(\mathrm{N}=24)$ acknowledged the impact of secondary trauma on themselves as they learned about trauma-informed practices for schools. And ChristianBrandt, Santacrose, and Barnett (2020) found $(\mathrm{N}=224$ teachers) that STS $(\beta=.03, \mathrm{p}=.081)$ and 
perceptions of trauma-informed care $(\beta=.02, \mathrm{p}=.127)$ were not associated with the "teachers' report of their intentions to leave the field of education due to stress" (p. 4). Trauma-informed policies and educational practices may have benefits for not only students but also for the stress levels of teachers. However, because trauma-informed education is not the topic of this dissertation, the information is offered as consideration for readers who might be interested in following trauma-informed care in education (Cole et al., 2005; Cole et al., 2013).

Studies of STS experienced by educators. There is, to date, no national STS study for educators in communities of trauma associated with opioid addiction and overdose. However, there is a limited number of STS studies on educators in different settings or with various stressors (see Table 2-10). Table 2-10 is organized chronologically to reflect the development of the STS construct and the research supporting secondary trauma. Although each study represented in the table has numerous findings, the findings selected for the chronological summary relate to teacher STS in communities of stress.

Table 2-10: Chronological Summary of Teacher STS Studies

\begin{tabular}{|c|c|c|c|c|}
\hline Study & $\begin{array}{l}\text { Study } \\
\text { design }\end{array}$ & $\begin{array}{l}\text { Sample Size, } \\
\text { Location, } \\
\text { Occupation }\end{array}$ & Findings & Implication \\
\hline $\begin{array}{l}\text { Robinson } \\
\text { (2005) }\end{array}$ & Survey & $\begin{array}{c}\mathrm{N}=184 \\
\text { Nova Scotia } \\
\text { and West } \\
\text { Virginia } \\
\text { Teachers, } \\
\text { counselors, } \\
\text { administrator } \\
\mathrm{s} \\
\text { (PK-12 } \\
\text { grade) }\end{array}$ & $\begin{array}{c}\text { Upper quartile } \\
\text { reported: } \\
26.09 \% \text { risk for } \\
\text { burnout } \\
33.15 \% \text { at risk for } \\
\text { compassion fatigue } \\
\text { Using initial multiple } \\
\text { regression analysis, } \\
\text { the criterion variable, } \\
\text { indirect work trauma }\end{array}$ & $\begin{array}{l}\text { This study is one of the } \\
\text { first studies to quantify } \\
\text { risk for compassion } \\
\text { fatigue for teachers. } \\
\text { The variable of indirect } \\
\text { trauma history has } \\
\text { persisted in STS } \\
\text { research; this means that } \\
\text { self-reports, across } \\
\text { disciplines, from helpers }\end{array}$ \\
\hline
\end{tabular}




\begin{tabular}{|c|c|c|c|c|}
\hline & & & $\begin{array}{l}\text { history, was } \\
\text { significant, } \mathrm{F}(1,150) \\
=5.717, p=.018 . \\
\text { The correlation for } \\
\text { Compassion Fatigue } \\
\text { and Impact of Events } \\
\text { Scale-Revised (IES- } \\
\text { R) for indirect trauma } \\
\text { was .580, also } \\
\text { significant }(p<.001) .\end{array}$ & $\begin{array}{l}\text { who have a trauma } \\
\text { history, are positively } \\
\text { associated with STS. } \\
\text { Rojas-Flores et al. (2015) } \\
\text { also confirmed this } \\
\text { finding, with teachers } \\
\text { with a trauma history } \\
\text { showing higher levels of } \\
\text { PTSD symptomatology } \\
\text { than those without a } \\
\text { trauma history } \\
\text { Here STS is described as } \\
\text { compassion fatigue. }\end{array}$ \\
\hline $\begin{array}{l}\text { VanBergeijk } \\
\text { \& Sarmiento } \\
(2006)\end{array}$ & $\begin{array}{c}\text { Interviews } \\
\text { Grounded } \\
\text { Theory }\end{array}$ & $\begin{array}{c}\mathrm{N}=28 \\
\text { United } \\
\text { States- } \\
\text { Mexico } \\
\text { Border } \\
\text { Teachers, } \\
\text { administrator } \\
\text {, other } \\
\text { professionals } \\
\text { Mandated } \\
\text { reporters of } \\
\text { child } \\
\text { maltreatment }\end{array}$ & $\begin{array}{c}\text { STSS subscales } \\
\text { endorsed, but scores } \\
\text { not reported } \\
\text { Three types of } \\
\text { symptoms emerged } \\
\text { from narratives (plus } \\
\text { physical symptoms): } \\
\text { 1. intrusive } \\
\text { symptoms: intrusive } \\
\text { imagery } \\
\text { 2. cognitive } \\
\text { symptoms: } \\
\text { powerlessness, doubt } \\
\text { of ability, avoidance } \\
\text { 3. emotional } \\
\text { symptoms: anxiety, } \\
\text { anger } \\
\text { 4. physical } \\
\text { symptoms: sleep } \\
\text { disturbance }\end{array}$ & $\begin{array}{l}\text { In qualitative research, } \\
\text { the narratives often } \\
\text { contain data that code } \\
\text { onto the three subscales } \\
\text { of intrusion, avoidance, } \\
\text { and arousal. } \\
\text { It might be necessary to } \\
\text { consider specialized STS } \\
\text { scales, depending on the } \\
\text { occupation and } \\
\text { occupational stressors. } \\
\text { Mandated reporters, even } \\
\text { in schools, have unique } \\
\text { stressors. }\end{array}$ \\
\hline
\end{tabular}




\begin{tabular}{|c|c|c|c|c|}
\hline & & & $\begin{array}{l}\text { Specific STS model } \\
\text { proposed for } \\
\text { mandated reporters }\end{array}$ & \\
\hline $\begin{array}{l}\text { Smith } \\
\text { Hatcher et al. } \\
(2011)\end{array}$ & Survey & $\begin{array}{c}\mathrm{N}=118 \\
\text { United States } \\
\text { Educators } \\
\text { and staff } \\
\text { Juvenile } \\
\text { justice } \\
\text { setting }\end{array}$ & $\begin{array}{l}\text { Full STSS Score } \\
\text { 37.74 } \\
76.3 \% \text { endorsed } \\
\text { intrusion symptoms } \\
\text { 39\% endorsed PTSD } \\
\text { symptoms } \\
\text { 81\% met at least one } \\
\text { core diagnostic } \\
\text { criteria for } \\
\text { posttraumatic stress } \\
\text { disorder } \\
\text { 55\% met two core } \\
\text { diagnostic criteria for } \\
\text { posttraumatic stress } \\
\text { disorder } \\
\text { 39\% met all three } \\
\text { core diagnostic } \\
\text { criteria for } \\
\text { posttraumatic stress } \\
\text { disorder } \\
\text { with is traumatized; severely } \\
\text { population they work } \\
\text { waid the students } \\
\text { wered or worse. }\end{array}$ & $\begin{array}{l}\text { When the full STSS } \\
\text { scores are reported, it is } \\
\text { useful to compare across } \\
\text { occupations and settings. } \\
\text { This score is a moderate } \\
\text { full score, right at the } \\
\text { edge of severe. } \\
\text { 39\% of the respondents } \\
\text { meeting the PTSD core } \\
\text { diagnostic criteria is a } \\
\text { high percentage. This is } \\
\text { the highest PTSD } \\
\text { assessment report (see } \\
\text { Table 2-9), and it is } \\
\text { higher than the global } \\
\text { PTSD prevalence, around } \\
10 \% \text { (95\% CI: } 8.1- \\
11.9 \% \text { ) for emergency } \\
\text { workers. } \\
\text { incarcerated youth with } \\
\text { one or more ACEs. } \\
\text { Bachte (2016) affirmed } \\
\text { more than 99\% of }\end{array}$ \\
\hline $\begin{array}{l}\text { Alisic et al. } \\
(2012)\end{array}$ & Survey & $\mathrm{N}=756$ & $\begin{array}{c}\text { 9-item Survey of } \\
\text { teachers (Supporting }\end{array}$ & $\begin{array}{l}\text { This is an international } \\
\text { study which added } \\
\text { information about the }\end{array}$ \\
\hline
\end{tabular}




\begin{tabular}{|c|c|c|c|c|}
\hline & $\begin{array}{l}\text { Random } \\
\text { sample }\end{array}$ & $\begin{array}{c}\text { The } \\
\text { Netherlands } \\
\text { Teachers }\end{array}$ & $\begin{array}{c}\text { children after } \\
\text { traumatic exposure) } \\
\text { Using multiple } \\
\text { regression analysis, } \\
\text { researchers found a } \\
\text { significant negative } \\
\text { association between } \\
\text { supporting children } \\
\text { exposed to trauma } \\
\text { and three variables: } \\
\text { 1. amount of teaching } \\
\text { experience ( } \beta= \\
\quad-.12 * * \text { ), } \\
\text { 2. whether teachers } \\
\text { had attended trauma- } \\
\text { focused training in } \\
\text { the past three years ( } \beta \\
=-.09 * *) \text {, and } \\
\text { 3. the number of } \\
\text { traumatized children } \\
\text { they had worked with } \\
\text { ( } \beta=-.10 * * \text { ) was } \\
\text { related to the amount } \\
\text { of stress }\end{array}$ & $\begin{array}{l}\text { need for teacher training } \\
\text { on trauma: } \\
51-63 \% \text { of the teachers } \\
\text { reported questions about } \\
\text { children's mental health } \\
\text { and where to get answers } \\
\text { about traumatic stress } \\
\text { Only } 9 \% \text { of the teachers } \\
\text { had any form of trauma- } \\
\text { related training }\end{array}$ \\
\hline $\begin{array}{l}\text { Wolf-Prusan } \\
(2014)\end{array}$ & $\begin{array}{c}\text { Survey } \\
\text { and } \\
\text { interview } \\
\text { Mixed- } \\
\text { Method }\end{array}$ & $\begin{array}{c}\mathrm{N}=146 \\
\text { surveys } \\
\mathrm{n}=16 \\
\text { interviews } \\
\text { California } \\
\text { High school } \\
\text { teachers }\end{array}$ & $\begin{array}{l}\text { The quantitative } \\
\text { findings were not } \\
\text { related to STS (they } \\
\text { focused on resiliency } \\
\text { in communities of } \\
\text { urban violence). } \\
\text { Report of interviews } \\
\text { mentions secondary } \\
\text { traumatic stress. }\end{array}$ & $\begin{array}{l}\text { The emotional toll of } \\
\text { secondary traumatic } \\
\text { stress on teachers is } \\
\text { present in qualitative } \\
\text { narratives. } \\
\text { Resilience is an } \\
\text { important addition in the } \\
\text { compassion } \\
\text { fatigue/secondary stress } \\
\text { conversation. There is } \\
\text { some support for the }\end{array}$ \\
\hline
\end{tabular}




\begin{tabular}{|c|c|c|c|c|}
\hline & & & $\begin{array}{l}\text { Qualitative findings } \\
\text { that might be salient } \\
\text { for opioid overdose } \\
\text { deaths in } \\
\text { communities: Violent } \\
\text { death of a student } \\
\text { (crime-related) } \\
\text { tended to modify } \\
\text { teachers' behaviors to } \\
\text { become more } \\
\text { relational in their } \\
\text { interactions with } \\
\text { current students. } \\
\text { Teachers also } \\
\text { reported secondary } \\
\text { trauma behaviors as } \\
\text { they had to put } \\
\text { student needs before } \\
\text { their own. }\end{array}$ & $\begin{array}{c}\text { notion that resilience } \\
\text { mediates the relationship } \\
\text { between compassion } \\
\text { fatigue and burnout } \\
\text { (Burnett \& Wahl, 2015) }\end{array}$ \\
\hline $\begin{array}{l}\text { Caringi et al. } \\
(2015)\end{array}$ & Survey & $\begin{array}{c}\text { N=229 } \\
\text { Northwestern } \\
\text { U.S. } \\
\text { Teachers and } \\
\text { school staff } \\
\text { (across six } \\
\text { public } \\
\text { schools) } \\
\text { First Nations } \\
\text { student } \\
\text { population }\end{array}$ & $\begin{array}{c}M=39.00 \text { SD } 13.70 \\
\text { on STSS } \\
75 \% \text { exceeded } \\
\text { subscale cutoffs on } \\
\text { all three subscales = } \\
\text { PTSD; therefore, they } \\
\text { might meet the } \\
\text { diagnosis of PTSD if } \\
\text { a standardized } \\
\text { assessment for PTSD } \\
\text { was administered } \\
\text { 35.3\% reported } \\
\text { moderate symptoms } \\
\text { of depression }\end{array}$ & $\begin{array}{l}\text { Moderate level of STS } \\
\text { for teachers, with some } \\
\text { in severe range. } \\
\text { The PTSD percentage is } \\
\text { also very high for this } \\
\text { study. It has been } \\
\text { postulated that the scores } \\
\text { are in the high range } \\
\text { because the student } \\
\text { population has higher } \\
\text { levels of stress. This } \\
\text { finding would concur } \\
\text { with Smith Hatcher et al. } \\
\text { (2011). } \\
\text { Caringi and other } \\
\text { researchers have }\end{array}$ \\
\hline
\end{tabular}




\begin{tabular}{|c|c|c|c|c|}
\hline & & & $\begin{array}{c}\text { Suggest Tier 1,2,3 } \\
\text { approach to } \\
\text { intervention and have } \\
\text { gone on to develop a } \\
\text { Tier } 1 \text { approach } \\
\text { (website) }\end{array}$ & $\begin{array}{l}\text { launched a five-module } \\
\text { training for educators, } \\
\text { based on their research. } \\
\text { STAT (Support for } \\
\text { Teachers Affected by } \\
\text { Trauma) is online and } \\
\text { free. } \\
\text { https://statprogram.org/tr } \\
\text { aining }\end{array}$ \\
\hline $\begin{array}{l}\text { Rojas-Flores } \\
\text { et al. (2015) }\end{array}$ & $\begin{array}{c}\text { Workshop } \\
\text { and } \\
\text { survey }\end{array}$ & $\begin{array}{c}\mathrm{N}=193 \\
\mathrm{~N}=257 \\
\text { Independent } \\
\text { samples } \\
\\
\text { Two studies } \\
\text { El Salvador } \\
\text { Educators }\end{array}$ & $\begin{array}{c}\text { Teachers with a } \\
\text { trauma history } \\
\text { showing higher levels } \\
\text { of PTSD } \\
\text { symptomatology than } \\
\text { those without a } \\
\text { trauma history } \\
\text { Study } 1 \text { with } \mathrm{N}=193 \text { : } \\
\text { 95\% CI }=.04-.16, z \\
=3.31, p=.0009 \\
\text { Study } 2 \text { with } \mathrm{N}=257: \\
95 \% \mathrm{CI}=.06-.31, z \\
=3.19, p=.001\end{array}$ & $\begin{array}{l}\text { The history of trauma in } \\
\text { the life of the preservice } \\
\text { educator would be one of } \\
\text { the critical factors to } \\
\text { explore in teacher } \\
\text { training, as it increases } \\
\text { the risk for developing } \\
\text { STS }\end{array}$ \\
\hline $\begin{array}{l}\text { Schepers } \\
(2017)\end{array}$ & $\begin{array}{c}\text { Survey } \\
\text { Interview } \\
\text { Mixed- } \\
\text { Method }\end{array}$ & $\begin{array}{c}\mathrm{N}=115 \\
\text { Phase 1: } \\
\text { Researcher- } \\
\text { designed } \\
\text { Attitude } \\
\text { survey on } \\
\text { STS } \\
\text { Surveys } \\
\text { n=10 } \\
\text { Phase 2: } \\
\text { Interviews }\end{array}$ & $\begin{array}{c}\text { The researcher } \\
\text { designed a survey, } \\
\text { specifically for } \\
\text { teachers: Teacher } \\
\text { Secondary Traumatic } \\
\text { Stress Scale } \\
\text { M=57 SD } 7.9 \\
\text { Range: possible range } \\
\text { 0-112 (28 questions, } \\
\text { 0-4 scale); actual } \\
\text { scored range: } 38-79 \\
\text { "Moderate level” }\end{array}$ & $\begin{array}{l}\text { Recommends that trauma } \\
\text { be reconceptualized or } \\
\text { broadened to include } \\
\text { insidious trauma in } \\
\text { schools and address STS } \\
\text { with pre-service teachers } \\
\text { Even though the survey } \\
\text { in this study cannot be } \\
\text { compared to surveys in } \\
\text { other studies due to lack } \\
\text { of standardization, } \\
\text { Schepers notes that all }\end{array}$ \\
\hline
\end{tabular}




\begin{tabular}{|c|c|c|c|c|}
\hline & & $\begin{array}{l}\text { Arkansas } \\
\text { Teachers }\end{array}$ & $\begin{array}{l}\text { Scores in relatively } \\
\text { normal distribution } \\
\text { (histogram) } \\
\text { No teachers reported } \\
\text { feeling "no STS" } \\
\text { STS noted in teacher } \\
\text { populations, describe } \\
\text { in } 6 \text { themes (emotion, } \\
\text { stress, safety, normal, } \\
\text { competent, and } \\
\text { resilience.) }\end{array}$ & $\begin{array}{c}\text { teachers report some } \\
\text { amount of STS. }\end{array}$ \\
\hline $\begin{array}{l}\text { Denham } \\
\text { (2018) }\end{array}$ & Survey & $\begin{array}{c}\mathrm{N}=172 \\
\mathrm{n}=84 \\
\text { High school } \\
\text { teachers, in } \\
\text { schools of no } \\
\text { school decay } \\
\text { (control } \\
\text { group) } \\
\text { n=88 } \\
\text { High school } \\
\text { teachers, in } \\
\text { schools of } \\
\text { school decay } \\
\text { United States }\end{array}$ & $\begin{array}{l}\text { Significant difference } \\
\text { in STS between } \\
\text { teachers in schools of } \\
\text { disrepair (termed } \\
\text { "blighted" by } \\
\text { Denham (2018) and } \\
\text { in literature) and in } \\
\text { control group ( } t=- \\
\text { 6.340, } p<.001, d f= \\
170) \\
\text { Significant difference } \\
\text { in anxiety symptoms } \\
\text { between two groups } \\
(t=-4.233, p<.001, \\
d f=132.757 \text { ) } \\
\text { Teachers employed in } \\
\text { school buildings } \\
\text { experiencing } \\
\text { disrepair ("blight") } \\
\text { then rated } 63.3 \% \text { ( } p< \\
.001, d f=170) \text { higher } \\
\text { on the STSS than } \\
\text { teachers in buildings }\end{array}$ & $\begin{array}{l}\text { These two findings } \\
\text { together may indicate a } \\
\text { higher risk for stress- } \\
\text { related mental health } \\
\text { issues for teachers, } \\
\text { especially in } \\
\text { communities of high } \\
\text { stress } \\
\text { This finding might } \\
\text { indicate that the } \\
\text { environment might also } \\
\text { have an effect, outside of } \\
\text { trauma. This lends } \\
\text { support to the notion of } \\
\text { insidious trauma, } \\
\text { stemming from the } \\
\text { environment (like with } \\
\text { poverty or } \\
\text { marginalization) as a }\end{array}$ \\
\hline
\end{tabular}




\begin{tabular}{|c|c|c|c|c|}
\hline & & & $\begin{array}{l}\text { of regular repair, with } \\
\text { no exposure to } \\
\text { students of trauma. }\end{array}$ & $\begin{array}{l}\text { variable for STS } \\
\text { (Schepers, 2017). }\end{array}$ \\
\hline $\begin{array}{l}\text { Welby } \\
\text { (2019) }\end{array}$ & $\begin{array}{l}\text { Multiple } \\
\text { case study }\end{array}$ & $\begin{array}{c}\mathrm{N}=76 \\
\text { Northeast } \\
\text { U.S. } \\
\text { Elementary } \\
\text { school } \\
\text { district } \\
\text { administrator } \\
\text { s, teachers, } \\
\text { counselors, } \\
\text { mental health } \\
\text { providers, } \\
\text { and } \\
\text { consultants }\end{array}$ & $\begin{array}{l}79 \% \text { of teachers } \\
\text { discussed secondary } \\
\text { trauma when asked } \\
\text { "How is the opioid } \\
\text { epidemic impacting } \\
\text { your } \\
\text { classroom/school" or } \\
\text { mentioned within the } \\
\text { interview } \\
\text { Need for } \\
\text { organizational } \\
\text { systems, preparation, } \\
\text { consistency, and } \\
\text { proactive plans to } \\
\text { support the schools, } \\
\text { administrators, } \\
\text { teachers, and students } \\
\text { impacted by the } \\
\text { epidemic }\end{array}$ & $\begin{array}{l}\text { This is the only } \\
\text { published study (non- } \\
\text { anecdotal) that could be } \\
\text { located dealing with the } \\
\text { opioid epidemic, } \\
\text { teachers, and mentioning } \\
\text { secondary traumatic } \\
\text { stress. } \\
\text { The anecdotal } \\
\text { information nationally is } \\
\text { overwhelming for STS } \\
\text { and teachers in } \\
\text { communities impacted } \\
\text { by the opioid epidemic. }\end{array}$ \\
\hline $\begin{array}{l}\text { Christian- } \\
\text { Brandt et al. } \\
(2020)\end{array}$ & Survey & $\begin{array}{c}\mathrm{N}=163 \\
\text { Pacific } \\
\text { Northwest } \\
\text { (United } \\
\text { States) } \\
\text { teachers in } \\
\text { underserved } \\
\text { schools } \\
\text { An online } \\
\text { survey } \\
\text { regarding } \\
\text { trauma- }\end{array}$ & $\begin{array}{c}\text { Trauma-informed } \\
\text { care perceived more } \\
\text { effective for students } \\
\text { by teachers with } \\
\text { higher rates of } \\
\text { compassion } \\
\text { satisfaction ( } \beta=.50, \\
p<.001) \text { and STS }(\beta \\
=.60, p=.001) \text {, and } \\
\text { lower rates of } \\
\text { burnout }(\beta=-.65, p= \\
.001),\end{array}$ & $\begin{array}{l}\text { Trauma-informed care } \\
\text { (TIC) and trauma- } \\
\text { sensitive schools (Cole et } \\
\text { al., 2013) is one } \\
\text { movement to assist } \\
\text { students with trauma. } \\
\text { Understanding how TIC } \\
\text { is perceived and } \\
\text { experienced by teachers } \\
\text { can help inform STS } \\
\text { intervention programs for } \\
\text { teachers. }\end{array}$ \\
\hline
\end{tabular}




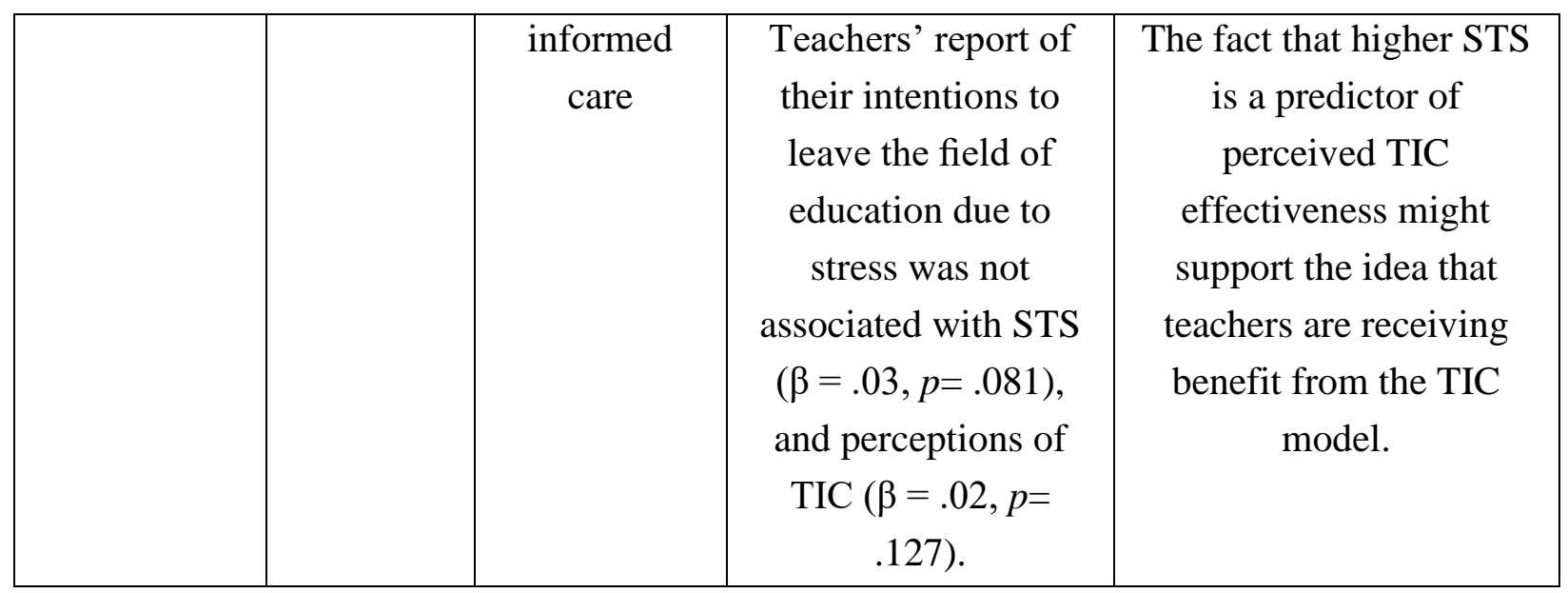

Of these studies, only four focused exclusively on teachers in public school settings (Christian-Brandt et al., 2020; Denham, 2018; Schepers, 2017; Wolf-Prusan, 2014). The studies supported the need for further research on teacher STS, especially in communities of stress (Denham, 2018; Smith Hatcher et al., 2011; VanBergeijk \& Sarmiento, 2006; Wolf-Prusan, 2014). The contention that the STSS is an appropriate survey for the study of teacher stress was supported (Denham, 2018; Smith Hatcher et al., 2011), even in studies that did not utilize survey methodology (VanBergeijk \& Sarmiento, 2006). National and international studies affirmed teacher STS, as did qualitative and survey methods.

Themes from the STS teacher studies. Schepers (2017) noted that the teachers studied concerning STS experienced STS in a normal curve, meaning that some experienced low levels of stress, while others experienced higher levels — with the majority of the teachers studied falling in the middle of the curve. Interestingly, no teachers reported experiencing no STSevery teacher felt some STS. Several themes emerged from the collection of studies: STS as a salient stressor, risk factor of previous trauma, and need for further training.

Impact of STS as salient stress for teachers. From the studies in Table 2-10, the construct of STS is shown as having an effect on teachers. Robinson (2005) explained that $33.15 \%$ of 
teachers are at risk for compassion fatigue, Smith Hatcher et al. (2011) noted a moderate level of STS for juvenile justice teachers (full STSS Score 37.74), while Caringi et al. (2015) found a total STSS score for teachers at a mean of 39.00. The difference between the two STSS scores of teachers, found by Denham (2018), supported the idea that difficult or stressful environments can impact teacher self-report of STS $(M=20.80$ for control group versus $M=34.03$ for teachers in high poverty schools).

Impact of previous trauma. Teachers who have a history of trauma in their background are more at risk for STS (Robinson, 2005; Rojas-Flores et al., 2015). Rojas-Flores et al. (2015) also confirmed that teachers with a trauma history showed higher levels of PTSD symptomatology than those without a trauma history. Researchers in other disciplines support indirect trauma findings. Lee et al. (2017) found that indirect traumatic events in firefighters (N $=212)$ increased the odds for posttraumatic stress symptoms $(A O R=1.93, p=.009$, Cohen's $d=$ .16). Akinsulure-Smith et al. (2018), through hierarchical linear regression, indicated the significance of previous trauma history $(B=1.02, p<.01)$ for refugee resettlement workers $(\mathrm{N}=$ 210). Cummings et al. (2018), with victim advocates $(\mathrm{N}=132)$, also supported being a past victim of trauma for prediction of STS symptoms through hierarchical regression analysis $(\beta=$ $.172, p<.01)$. The findings with teachers are additionally confirmed through the findings of researchers with other helping professionals.

Impact of need for training. In light of the effects of STS on teachers, researchers noted the need for more training for educators. Alisic et al. (2012) found that only $9 \%$ of teachers had received training in the past and that training was negatively associated with STS $(\beta=-.09)$. Welby (2019) described the teachers' needs for planned programs to deal with the impact of secondary stress. Brady et al. (1999) noted that mental health worker caseload had a stronger 
relationship to STS trauma symptoms; this could be salient because teachers have many students per day (caseload) for a prolonged time (continuous). Caringi et al. (2015), the developers of the first national training program in STS for teachers (STAT: Support for Teacher Affected by Trauma, 2019), supported a three-tiered level of STS intervention for teachers mainly because of the time teachers spend with traumatized children each day. Caringi et al. (2015) noted that "the dose-response theory of direct trauma exposure may be extrapolated to secondary trauma" (p. 39) and applied to public school personnel. Training programs, plus intervention and supports, might be most useful for teachers.

\subsection{Secondary Traumatic Stress in High Opioid Epidemic Zones}

In addition to the body of research on educators with STS, there is a minimal amount of information regarding teachers in areas of high opioid impact. While most of the information is anecdotal from media accounts, some data are from research. First, the limited research will be examined, and then the anecdotal reports will be briefly explored.

Research accounts. Two exceptions to the media anecdotal collection are Welby (2019) and Anderson et al. (2019). Welby (2019), in a multiple case study ( $\mathrm{N}=76$ ) of educators dealing with the opioid epidemic, referenced the narratives of the teachers as they described the impact of STS. Anderson et al. (2019), in a survey of 2,205 teachers in West Virginia for University of West Virginia, reflected that while "Virginia teachers report an increase in students impacted by substance use in the home, only 10 percent of teachers feel confident in knowing how to support children with parents or caregivers who use substances." The study by Welby and the survey by WVU are two initial non-anecdotal research-based accounts.

When Welby (2019) asked teachers about the impact of the opioid epidemic on them, from $58 \%-100 \%$ of teachers (depending on the school site) discussed secondary trauma. Welby 
described STS as an unanticipated theme, yet a prominent one, reflecting that not only is the trauma response of the students impacting the teachers, but the additional unmet needs of the students are depleting the teachers. Welby (2019) noted the emotional toll on the teachers, "Throughout the interviews, teachers told stories about the impact the epidemic is having on their students with tears in their eyes, some could not finish sentences, some changed the subject quickly when they did not want to talk about it anymore" (p. 111). Welby also found that $79 \%$ of teachers $(\mathrm{N}=76)$ felt unprepared to teach children exposed to the opioid epidemic, and $92 \%$ of teachers reported that they had received no training or professional development focused on students impacted by the opioid epidemic (p. 200).

After surveying 2,205 teachers in West Virginia, Anderson et al. (2019) found that "emotional exhaustion, cynicism and a lack of personal accomplishment related to the changing classroom dynamics created by the opioid crisis" was indicated by over $70 \%$ of survey responses, with $30 \%$ of teachers stating that they experienced burnout frequently. Additionally, over 35\% of teachers highlighted "significant increases" in the number of students who were impacted by addiction issues in the home, notably with parent or caregiver. Many teachers noted a marked mismatch between behaviors in the classroom (such as erratic attendance, irresponsibility, or low motivation) and their confidence with feeling equipped or prepared to cope with these behaviors, which they see as increasing.

Media accounts. Anecdotally, Litvinov (2019) described NEA interviews with ten educators from West Virginia and Ohio, two states that are identified as "hardest hit and longestsuffering" on the opiate map. Educators' narratives enumerated issues with a striking similarity to those noted by Anderson et al. (2019): losing sleep, a lack of response to typical teaching strategies, schools as safety nets, emphasis on addressing physical and mental needs of children, 
a lack of formal training. Because these are anecdotal accounts, no data are backing up these accounts-yet. As of now, these are narrative accounts for national media sources.

While teachers describe these emotion-laden reactions, there are few emotion-based supports for teachers. There are, however, content-based supports. Schorchit (2017) revealed that in some states, drug education begins in kindergarten. Blad (2019) reported that the American Federation of Teachers (AFT), in collaboration with Harvard Medical School, has developed an online course to assist educators, nurses, social workers, and other educational stakeholders with understanding the opioid epidemic. Nadiv (2019), detailing the need to support teachers and staff, addressed providing teachers with content-based supports by "providing them with information about trauma and the behaviors that are common among children who have experienced it, and by giving them practical trauma-informed practices to use" (para. 5) but not emotion-based supports. While these efforts of educating children and educating teachers are beneficial, they do not explicitly address the notion of secondary traumatic stress that can develop from supporting students of trauma. There is a need for research-based information to support these anecdotal accounts.

\subsection{Gaps in the Literature}

Research and theory can help form a complete understanding of trauma; however, the picture is incomplete because there are noticeable missing pieces in the literature. These gapsinvolving terminology, roles of teachers, and trauma - serve to muffle necessary conversations in the areas of teachers and secondary traumatic stress.

\subsubsection{Overlapping Terminology}

One area that needs continual work is the discrimination between burnout and STS. The findings are somewhat contradictory. Kassman-Adams (1999) explicitly found that vicarious 
stress was not related to occupational stress. However, Devilly et al. (2009) noted that "burnout, with its basis in work-related stressors, is the strongest predictor of therapist distress" (p. 383), which could imply that the claims of STS might be overestimated because they can be more clearly explained by burnout. STS literature can seem to be an inadvertently small sample of traumatology research literature due to the terminology. Another area that needs more consideration has been raised by Nimmo and Huggard (2013), who noted that STS research for physicians is severely limited because most research in this area is completed with the terminology "compassion fatigue" or "vicarious trauma." There may be definition distortion, which would require greater clarity of definitions to parse further the differences between compassion fatigue, vicarious trauma, and secondary stress. Finally, Hensel et al. (2015) reported that risk factor association in the STS literature for caseload frequency and personal trauma decreased with the advancing year of study publication (2008+), which means that the conversations about risk factors and STS might need to be approached with caution and more data.

\subsubsection{Multiple Roles of Teachers}

Although most empirical work on STS has been completed with psychotherapists, mental health professionals, and trauma therapists (Brady et al., 1999; Bride, 2007; Pearlman \& Mac Ian, 1995), it is less understood with teachers as a population (Landers, 2018; Schepers, 2017). Teachers are often seen through the lens of instructional, policy, and collaborative or association leadership (Barnett et al., 2018). In classrooms, though, educators are also the first responders to children's emergencies (Denham, 2018). Outside of the classroom, teachers are encouraged to "attend to both parent education and parent involvement" (Murphy \& Tobin, 2011, p. 37). 
Additionally, Denham (2018) noted that teachers often live in the communities in which they work; therefore, if there are negative community impacts, the teachers will also experience these.

With these expanding roles and this increasing load, it is crucial to consider the impact of community stressors on the entire community, including schools, students, and teachers. To respond to these needs, school-wide systems of support offer a collaborative network for school community response: for academic performance, there is Response to Intervention (RTI; Fuchs \& Fuchs, 2006); for social and behavioral supports, the School-wide Positive Behavior Interventions and Supports system (SWPBIS; Sugai \& Horner, 2002); and for a more comprehensive and integrated model, there is the three-tiered model of prevention (Lane, Oakes, $\&$ Menzies, 2010). There is support for the notion that school-wide systems of support are associated with teachers' perceptions of efficacy and burnout. Ross, Romer, and Horner (2012), using multi-level regression $(\mathrm{N}=184)$, found support for their model showing significantly lower levels of burnout and significantly higher levels of efficacy for teachers using school-wide positive behavioral interventions and supports $(\chi 2(3)=8.16, p=.042$.$) .$

School-wide systems of support with multi-tiered models exist to support students more holistically and comprehensively. For teachers, though, Hydon, Wong, Langley, Stein, and Kataoka (2015) reflected that "teachers can find themselves in the role of key person in identifying the social-emotional needs of students and recognizing when these traumas affect their ability to learn" (p. 322). Hydon et al. continued by clarifying that, for 5-7 hours per day, teachers interact with children, through instruction and care; therefore, through this prolonged exposure to traumatized children, the teachers might experience the construct of indirect or secondary traumatic stress. Berger et al. (2016) described the work of teachers with students of 
trauma in schools as the reality of "shared trauma." The school-wide systems of support might reduce exposure but will not necessarily eliminate it.

Teachers are also considered possible providers of mental health preventions. In a metaanalysis ( $=49)$, Franklin, Kim, Ryan, Kelly, and Montgomery (2012) found that teachers not only provided mental health interventions as sole providers $(18.4 \%)$ but also that teachers were actively involved as partners in $40.8 \%$ of mental health interventions overall. Within the cannon of STS research literature, social workers, psychologist, and emergency workers teachers are typically conceptualized as helpers; however, Borntrager et al. (2012) argued that teaching should be included in the list of helping professions and, therefore, considered for research like STS with helping professionals.

\subsubsection{Trauma}

Albaek, Kinn, and Milde (2017) noted a limitation in the willingness of professionals to identify and explore trauma in children. This reluctance motivates a more in-depth understanding, not only of trauma experiences but also of professionals' experiences in addressing the trauma responses of children (Albaek et al., 2017). Research in this area is limited, and even counseling and medical professionals seem to display beliefs and attitudes that might narrow how childhood trauma is addressed (Albaek et al., 2017). Studies from school settings that utilized the perspectives from other helping professional backgrounds-like counseling (Sikes, Walley, \& Hays, 2013) or medicine (Coker et al., 2000)—have been considered.

This dissertation has the potential to address some of the gaps identified in the literature review. First, by using the STSS and carefully discriminating STS from burnout, there is a potential to increase the body of findings of STS. Because of the use of the STSS and its 
correspondence with the PTSD DSM-IV diagnostic framework, the results will be linked to STS and not compassion fatigue or vicarious stress. Second, based on education, STS, and opioid research in schools, the role of teachers continues to develop. This research can add support to that area by looking at the self-report of teachers' stress. Finally, this study will add to the conversation about teachers' attitudes about the trauma of students in communities of stress.

While these gaps will not be fully addressed, this study will touch on each of these areas.

\subsection{Conclusion}

The mechanism of secondary stress is vital to our understanding to address the research questions on the secondary traumatic stress of teachers in communities impacted by the opioid epidemic (see Figure 2-3).

Figure 2-3: Mechanism of Secondary Traumatic Stress in Opioid-Impacted Communities

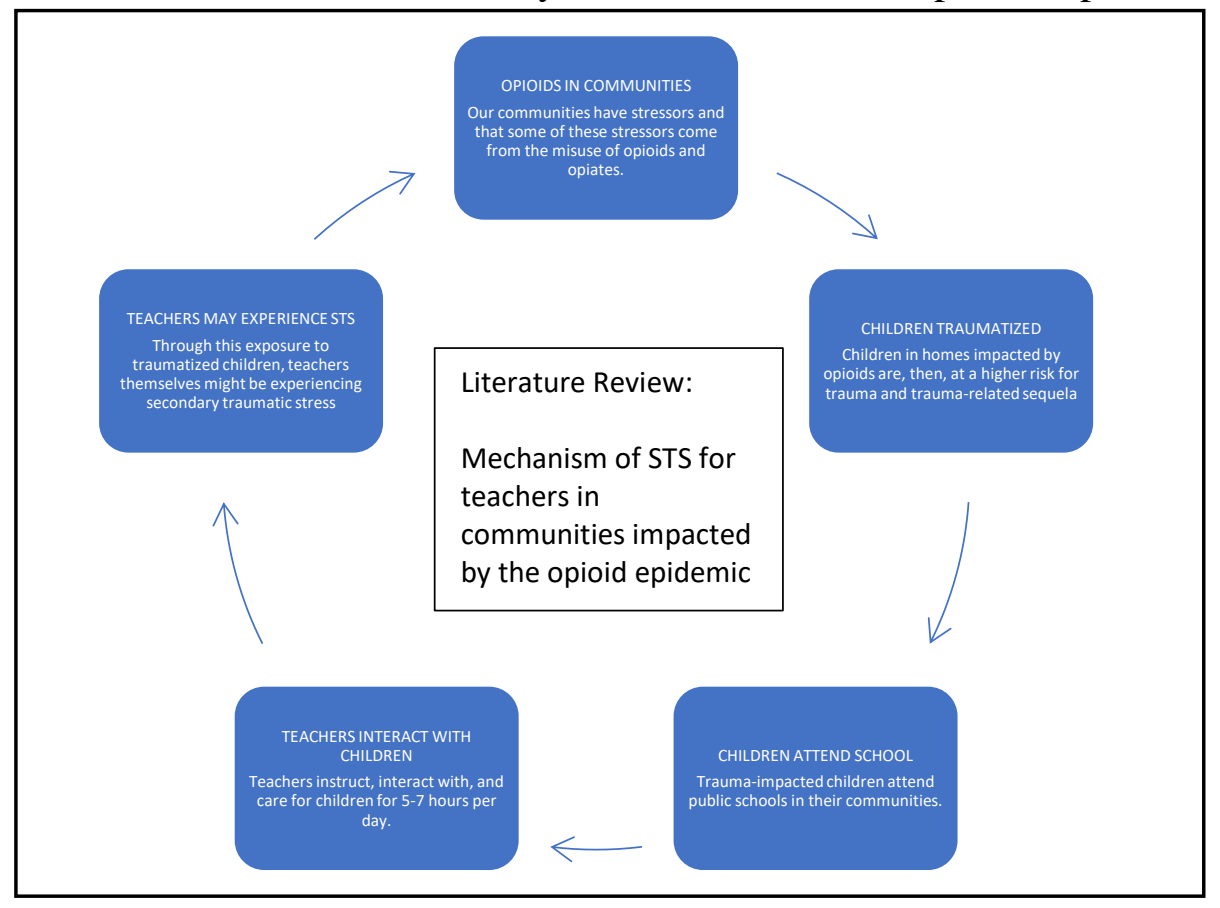

Communities impacted by opioids are not merely dealing with adults who take too many prescription drugs. The opioid epidemic has transformed from a concern about drugs, overprescribing, or isolated people in rural communities to a social and educational crisis. An 
average of 115 people die per day from opioids, which is an increase over five times the death rate in 1999; of drug-related deaths, 66\% involve an opioid (Centers for Disease Control and Prevention, 2020b). Although there is some fluctuation state-to-state (Quast, 2018), the opioid crisis is the single driving force behind the increase in foster care placements nationally (Radel et al., 2018b), leaving a mixed picture of foster care but a clear picture of risk. School-aged children are particularly impacted. As a result, schools are equally affected by the changes in families and communities because of opioids (Klein, 2018). As the opioid epidemic increases (Jones, Logan, Gladden, \& Bohm, 2015), communities and schools are impacted by the outcome with issues like addiction, overdose, crime, neglect, rise in foster care, increased medical care, and death (Hefling \& Stratford, 2018; Radel et al., 2018).

Teachers have been tasked with additional intervention/prevention duties, especially in communities that have a high prevalence of opioid use (U.S. Department of Education, 2018). With rising foster care and death rates that are adding to an already difficult teaching profession, school administrators and teachers are sharing anecdotes about increasing stress, feelings of hopelessness, and grief (Reilly, 2018). Teachers are working as secondary responders to trauma in a prolonged crisis (Landers, 2018). There is a paucity of information in this area to help researchers understand how teachers who are responding to the opioid epidemic are experiencing their feelings and symptoms of secondary stress (Landers, 2018).

Proceeding from this assumption, children in homes impacted by opioids are, then, at a higher risk for trauma and trauma-related sequela. Although not all children will be affected in the same ways, children in homes affected by opioids are at a higher risk for traumatic experiences. Trauma-impacted children attend public schools in their communities. La Greca et al. (2008) and others approximate one-fourth of school-age children as trauma-impacted before 
the age of 16, while Copeland et al. (2007) have placed that percentage between 50-60\% with the use of longitudinal study. Statistics on children and the opioid epidemic intersect this information on trauma (Swartz, 2018): the percentage of infants born with neonatal abstinence syndrome (NAS) increased over 300\% between 1999 - 2013 in 28 states; opioid poisonings in toddlers and preschoolers increased $205 \%$ during this same time; the once-declining teen death rate from overdose reversed in 2015 (Edelman, 2017); 30-40\% of children in kinship foster homes are placed due to parental substance abuse (Collier, 2018; Edelman, 2017); students in K-12 settings are at increased risk for traumatic experiences with accompanying physical and mental health problems as they develop (Swartz, 2018). The number of children under age 20 who live in counties with high overdose death rates rose from less than 250,000 to over 22 million between 2000 and 2016; this increased the percentage of children in these counties with overdose death rates from less than $1 \%$ to $28 \%$ (Mather, Jarosz, \& Slowey, 2019). Kelly, Harvard Medical School associate professor of psychiatry in addiction medicine, identified children as a neglected subpopulation, in need of more study (Collier, 2018). Children in this subpopulation already experience increased risk of family disruption, as well as increased risk of trauma experiences and adverse life experiences (ACEs), which have lifelong consequences for physical and mental health, especially if left untreated (Normile et al., 2018). Because of this growing risk profile for school-age children coping with trauma, teachers are at prolonged and continuous risk for developing STS (Motta, 2015). There is a need for research that examines STS in teachers in our communities impacted by the opioid epidemic. 


\section{Chapter Three: Methodology}

The purpose of this study was to investigate if K-12 teachers' self-reported level of secondary traumatic stress (STS) relates to their perceptions of trauma in their school communities. This chapter presents the research questions, the research design, the sampling strategy, the instrumentation, data collection procedures, and the statistical analyses for the dissertation research.

\subsection{Research Questions and Design}

- RQ1: What are K-12 teachers' self-reported levels of secondary traumatic stress?

- RQ2: Is there a relationship between teachers' self-reported levels of secondary traumatic stress when teachers are from states of high-opioid impact, states of medium-opioid impact, and states of low-opioid impact?

- RQ3: Is there a relationship between self-reported levels of secondary traumatic stress and the characteristics of teachers?

- $\quad$ RQ3a: Is there a relationship between self-reported levels of secondary traumatic stress and the age of teachers?

- $\quad$ RQ3b: Is there a relationship between self-reported levels of secondary traumatic stress and gender?

- $\quad$ RQ3c: Is there a relationship between self-reported levels of secondary traumatic stress and race/ethnicity?

- $\quad$ RQ3d: Is there a relationship between self-reported levels of secondary traumatic stress and relationship status?

The independent variables for the study are the low, medium, and high opioid epidemic zones, along with the demographic characteristics age, gender, race/ethnicity, and relationship status, as well as the teachers' perceptions of ACEs among their students. The dependent variables for the study are the teacher STSS scores, and the STSS subscale scores of intrusion, arousal, and avoidance. 
The purpose of the study was to understand the levels of secondary stress among K-12 public school teachers in communities with varying opioid mortality rates. The research design was both correlational and descriptive. The correlational aspects of the study sought to determine relationships among variables (Creswell \& Guetterman, 2018). According to Issac and Michael (1995), the purpose of descriptive research is "to describe systematically the facts and characteristics of a given population or area of interest factually and accurately" (p. 50). It was non-experimental because no pre- and post-test design was employed, and there was no attempt to predict causation.

\subsection{Sampling Strategy}

The population for this study was comprised of K-12 public school teachers in the continental U.S. The participants for this study were purposefully recruited according to the following five requirements: respondents needed to (a) have access to the internet for participation in the online survey; (b) be able to comprehend written English; (c) be over the age of 18; (d) be teaching in one of the geographic areas determined by the parameters of the study; and (e) be teaching for at least one year.

Non-probability sampling was administered under the direction of an assigned Qualtrics project manager. Utilizing information from the National Institute of Health (NIH) study by Kiang et al. (2019), three different areas of opioid impact were determined by intersecting mortality rates (MR) and annual percentage of change concerning the rising mortality rate (APC). In the NIH study, low mortality rate (per 100,000) was determined as 0.0-5.0, medium as 5.0-10.0, and high as greater than 10.0. A slow annual percent of change (of the mortality rate) was defined as 0-26\%, moderate as 26-41\%, and rapid as greater than $41 \%$ (Kiang et al., 2019). Kiang et al. (2019) identified opioid hotspots as areas where the mortality rate is both high 
(>10.0) and rapidly increasing (41\%). The intersection of the two rates determines if the community is considered high, medium, or low impact.

The NIH cross-sectional study $(\mathrm{N}=351,630)$ identifying the changes in the geographic distribution of opioid mortality across the United States examined the rates for any opioid, heroin, synthetic opioids, and natural and semisynthetic opioids (Kiang et al., 2019). The data were reported, by state, for each opioid category. For this STS teacher study, I selected "any opioid." It should be noted that the MR and APC vary by state, and by opioid type. The selection of "any opioid" was intentional because it was the broadest category. After selecting the opioid designation, I needed to determine three opioid zones to select states for the study. The low opioid epidemic zone required states with the lower MR and APC rates, while the high opioid zone required high MR and APC rates.

To select states for the study that represented three different and discrete levels of opioid mortality, I relied on the information from the NIH study as a guideline. The NIH study looked at various changes in mortality as distributed geographically in the United States, using the parameters of rapid and slow, as well as low and high mortality (Kiang et al., 2019). I used similar ranges for my designations of low, medium, and high, which are based on the NIH study but have no overlap of ranges. Avoiding an overlap of the range was necessary, so states do not fall into two different categories.

Three discrete and non-intersecting levels for the study were determined by adjusting the parameters to identify three distinct and non-overlapping opioid impact zone ranges. The highly specific parameters defined each of my three opioid impact zones (see Table 3-1). 
Table 3-1: Comparison of Mortality Sample Range

\begin{tabular}{|l|c|c|}
\hline $\begin{array}{c}\text { Epidemic } \\
\text { Designation }\end{array}$ & $\begin{array}{c}\text { Range Percentage } \\
\text { NIH Study }\end{array}$ & $\begin{array}{c}\text { Range Percentage } \\
\text { STS Teacher Study }\end{array}$ \\
\hline Mortality Rate (MR) & & $0.0-5.0$ \\
\hline Low & $0.0-5.0$ & $5.1-7.3$ \\
\hline Medium & $5.0-10.0$ & $>18.5$ \\
\hline High & $>10.0$ & \\
\hline & & \\
\hline $\begin{array}{l}\text { Annual percent of } \\
\text { change (APC) }\end{array}$ & $0-26$ & $0-2.2^{\mathrm{a}}$ \\
\hline Slow & $26-41$ & $18.1^{\mathrm{b}}-23$ \\
\hline Moderate & $>41$ & $>30^{\mathrm{b}}$ \\
\hline Rapid & & \\
\hline
\end{tabular}

${ }^{a}$ The range in this category reflects the greatest discrepancy between the NIH study and this study. This was done to assure a clear delineation between the low opioid mortality states and the medium opioid mortality states.

${ }^{\mathrm{b}}$ This number falls below the NIH category.

As shown above, four of the six ranges for this dissertation (STS Teacher Study) fall within the same range scale as the NIH study. In the mortality rate category, all of the states fall within the same range as the NIH study. In the APC category, the slow range falls within the same range as the NIH study; however, for the moderate and rapid categories, I started slightly below the range to include more states in the study. Care was taken that there was no overlap in categories; this way, each state fit discretely into one category only and would be counted as either low, medium, or high, with no overlap or question.

Kiang et al. (2019) noted that the range of parameters could be adjusted depending on the need of the researcher, and the online interactive tool provided by the NIH authors was used for this purpose. I wanted a more extensive range between the states than those represented by the pre-determined parameters in the NIH study. Kiang et al. (2019) established a method for researchers to adjust the definition of rapid and slow increases, as well as low and high mortality. I refined the parameters for this study to identify low, medium, and high mortality states. Hence, the low mortality states are sufficiently low, which gives them a discretely different profile from 
the medium mortality states. However, even with this adjustment, the low states fell within the same range as the NIH study. The only ranges that veered slightly from the NIH study were the APC for the medium and high states. I did this so more medium and high opioid epidemic states could be included in the study, with a clear delineation between what defines a medium state and what defines a high state.

To select states with low opioid mortality and a low annual percent of change, I selected states from the NIH study from the lowest end of the range (see Table 3-1). By lowering the annual percentage of change, I selected states with the lowest opioid impact (California, Montana, South Dakota, Texas). The medium-impact states (Alabama, Arizona, Colorado, Delaware, Georgia, Louisiana, Minnesota, Mississippi, Missouri, New Jersey, North Carolina, South Carolina, Tennessee, Wisconsin) follow the NIH ranges with the slight lowering of the APC rate, as do the high impact states (Connecticut, Florida, Maine, Maryland, Massachusetts, New Hampshire, Ohio, Pennsylvania, Washington, D.C.). Schools in three geographic categories meet the requirements of the research design:

- Low Opioid Mortality States: California, Montana, South Dakota, Texas $\mathbf{M R} \leq \mathbf{5 . 0} \cap \mathbf{A P C} \leq \mathbf{2 . 2}$ The mortality rate is less than or equal to 5.0\%, intersecting with an annual percentage of change in mortality rate less than or equal to $2.2 \%$.

- Medium Opioid Mortality States: Alabama, Arizona, Colorado, Delaware, Georgia, Louisiana, Minnesota, Mississippi, Missouri, New Jersey, North Carolina, South Carolina, Tennessee, Wisconsin

$\mathbf{5 . 1} \leq \mathrm{MR} \leq 7.3 \cap \mathbf{1 8 . 1} \leq \mathrm{APC} \leq \mathbf{2 3 \%}$ The mortality rate is greater than or equal to $5.1 \%$ but less than or equal to $7.3 \%$, intersecting with an annual percentage of change in mortality rate greater than or equal to $18.1 \%$ but less than or equal to $23 \%$.

- High Opioid Mortality States: Connecticut, Florida, Maine, Maryland, Massachusetts, New Hampshire, Ohio, Pennsylvania, Washington, D.C. 
$\mathbf{M R} \geq \mathbf{1 8 . 5} \cap \mathbf{A P C} \geq \mathbf{3 0} \%$ The mortality rate is greater than or equal to $18.5 \%$, intersecting with an annual percentage of change in mortality rate greater than or equal to $30 \%$.

Qualtrics (2014) has a rigorous procedure for assisting researchers with sample populations, which they described for researchers:

Qualtrics panel partners randomly select respondents for surveys where respondents are highly likely to qualify...Each sample from the panel base is proportioned to the general population and then randomized before the survey is deployed. (p. 4)

In addition to the selection processes, there are also processes which guard against bias, "Potential respondents are sent an email invitation. To avoid self-selection bias, the survey invitation does not include specific details about the contents of the survey" (p. 5). The sampling strategy is purposive (Ruel et al., 2016) in that the respondents were selected because, as teachers, they have specialized knowledge of teaching and issues about students. While the sampling procedure is nonrandom (Lodico, Spaulding, \& Voegtle, 2010), possible replication of the purposive sampling strategy outlined above could enhance generalization and transferability (Polit \& Beck, 2010). Specialized knowledge of teaching was required for the survey, and a large sample of teachers was gathered. Ruel et al. (2016) stated that meeting these two conditions increases the likelihood of producing excellent data from the survey result.

Public school K-12 teachers in the continental United States were recruited through Qualtrics. Qualtrics utilized an incentive program to ensure $n=150$ complete surveys in each of the three opioid-impact areas $(\mathrm{N}=450)$. Qualtrics allowed for the posting of the questionnaire, participant access to the instrument, and researcher retrieval of the results securely and confidentially. A sample description, reflecting the demographic information about the survey 
respondents, with the descriptive details, can be seen in Table 4-2. The 450 surveys that were returned were $100 \%$ completed.

\subsection{Instrumentation}

For the current dissertation research, the questionnaire was developed using the Secondary Traumatic Stress Scale (STSS; Bride et al., 2004). Permission was obtained for this scale (see Appendix C). By adding demographic and open-ended questions to the STSS, I designed a survey for the dissertation called Secondary Traumatic Stress of Teachers in School Communities Impacted by the Opioid Epidemic (see Appendix B). The questionnaire is a combination of three pre-survey questions, which included consent, age default (meaning that if a respondent did not meet the age requirement, the survey ended for the respondent), and teacher default (meaning that if a respondent did not meet the teacher-question requirement, the survey ended for the respondent). If any of these questions were not answered appropriately, respondents were deselected from the survey process. The questionnaire contained nine demographic items, a question providing a 14-item drop-down scale designed to measure adverse child experiences in students, (PHL-ACE; Health Federation of Philadelphia and Philadelphia ACE Research and Data Committee, 2012), a 17-item scale designed to measure secondary traumatic stress (STSS; Bride et al., 2004), and four open-ended questions. The questionnaire concluded with a section of stress-related resources for respondents.

Demographic questions. The first section, comprised of descriptive demographic questions, queried age, gender, race/ethnicity, marital status (on the research question, this is listed as relationship status), grade level taught, setting and years of teaching, and one geographic question (U.S. state of survey origin). These were used to sort the responses into low, medium, and high groups based on the opioid designation. Care was taken to reflect a 
comprehensive list of possible options that a respondent would like to select (Ruel et al., 2016) as this improves participant responsiveness.

Adverse Childhood Experiences Scale. The next section of the questionnaire utilized a multiple-answer section, in drop-down format, regarding student trauma experiences based on an expanded version of the Adverse Childhood Experiences (ACE) scale (Felitti et al., 1998). Adverse childhood experiences (ACEs) are hazards in the environment to the development of children psychosocially and cognitively (Leeb, Lewis, \& Zolotor, 2011). The ACEs scale is necessary because this study is not examining teacher trauma; instead, it is exploring teacher secondary trauma. The construct of STS assumes that teachers are responding to issues of student trauma response in their classrooms. The ACEs scale was used to assess this assumption. ACEs include the following: physical or emotional abuse or neglect, loss of a parent, divorce or discord in the family, exposure to alcohol or drug abuse and mental illness in the home, or violence in the home or neighborhood (Bethell et al., 2017). In collaboration, the U.S. Centers for Disease Control and Prevention (CDC) and medical professionals from Kaiser Permanente in Southern California developed the initial ACEs survey, winnowing 17 items (Felitti et al., 1998) to the more common 11-item instrument used currently.

Through the Behavioral Risk Factor Surveillance System Survey (BRFSS), a large sample of adults $(n=186,423)$ responded to these 11 questions through the CDC's administration of the survey (Centers for Disease Control and Prevention, 2009, 2010, 2011, 2012). The survey is scored by totaling the number of adverse experiences a person self-reports or a parent or guardian reports as a trauma exposure for a child (Bethell et al., 2017). This type of scoring is called cumulative scoring in that it does not seek to rate the severity of the traumatic 
experiences; instead, the "dose-response effect consistently emerges in research irrespective of the specific ACEs involved" (Bethell et al., 2017, p. S52).

By assessing results from the National Survey of Children's Health (NSCH), Bethell et al. (2017) examined the internal validity across 14 ACEs used in medical and mental health settings; this included the Philadelphia Childhood Adversity Questionnaire and the Philadelphia Urban ACEs Study. The researchers reported that a single-factor model was a good fit for the data (root mean square error of approximation $1 / 40.01$; comparative fit index 1/4 0.99; TuckerLewis Index 1/4 0.98; chi-square 1/4 312.84; $\mathrm{n} 1 / 4$ 94,520; $p<.01$ ). The validation provides the justification for the three additional items incarcerated household member, felt racial/ethnic discrimination, and lived in foster care to be added to the survey as drop-down selection categories for adverse experiences. These experiences may or may not lead to trauma, based on the PHL-ACE categories (Health Federation of Philadelphia and Philadelphia ACE Research and Data Committee, 2012).

Although ACEs typically rely on adults to report retrospectively on their own experience, there is precedent for education professionals to assess the prevalence of ACE exposure in students (Blodgett \& Lanigan, 2018). Blodgett and Lanigan (2018), surveyed school personnel about the records of over two thousand de-identified students $(\mathrm{N}=2,101)$ in public $\mathrm{K}-6$ schools. The results of these teacher ratings revealed correlations between the number of ACEs as identified by teachers and the risk of poor school attendance, behavioral issues, and standards of academic success. Several of the findings included that students with identified attendance problems had a higher ACE score that was significant $(M=1.8 ; S D=1.3)$ compared to students without attendance concerns $(M=0.8 ; S D=1.9)$. As the number of school performance concerns increased, the researchers also noted that the mean ACE scores of children also increased F (1, 
$2,098)=169.9, p<.0001)$. Blodgett and Lanigan (2018) asserted that using educators in this way is likely to produce an under-reporting of adversity. The precedent of Blodgett and Lanigan provided support for my survey in which teachers were asked to consider the types and prevalence of student trauma for students in their classrooms. For this study, the data were used primarily to provide descriptive information about teachers' perceptions of the types of adverse events experienced by their students (see Table 4-13).

Secondary Traumatic Stress Scale. The primary research question was addressed with the Bride et al. (2004) 17-item Secondary Traumatic Stress Scale (STSS). Bride et al. (2004) developed the STSS in response to a lack of instrumentation to measure secondary trauma in providers of supportive services for trauma-survivor clientele. The 17 items are measured on a Likert type scale of $1=$ never, $2=$ rarely, $3=$ occasionally, $4=$ often, and $5=$ very often.

In addition to measuring overall stress, the instrument measures intrusion, avoidance, and arousal symptoms, which are associated with the experience of secondary trauma. Intrusion is characterized by reoccurring or intrusive recollections, while avoidance is described as efforts to avoids feelings, thoughts, or reminders of traumatic events; arousal is indicated by anxiety or increased anger or difficulty concentrating (American Psychiatric Association, 2000; Bride, 2007). The STSS is designed to measure secondary stress in each of these three dimensions identified on the DSM-IV for PTSD. Refer to Appendix B for the STSS.

There are three ways to score the STSS (Bride, 2007). These include the following: 1) summed percentile, 2) cutoff score, and 3) algorithm approach. All three methods were utilized in this study and then compared. The summed percentile method, the most common method, follows this procedure: each of the 17 questions is given a score (1-5), corresponding to the respondent's answer on the Likert scale $(1=$ never, $2=$ rarely, $3=$ occasionally, $4=$ often, and 5 
$=$ very often). The scores are then summed to obtain a total score (Bride, 2007). The higher the score, the more severe the STSS. Jacobs et al. (2019) stated that if the total is below 28, little or no STS is reflected. If the score is between 28 and 37, mild STS is indicated. A score between 38 and 43 signifies moderate STS, while a score between 44 and 48 shows a high level of STS. Severe STS is indicated by scores over 49 . These scores are then compared to percentiles (see Table 2-6).

A more straightforward scoring method, the cutoff method, utilizes the score of 38 as a cutoff: if a total score on the STSS is 38 or higher, STSS is indicated (Bride, 2007; Bride et al., 2007). The algorithm method examines the number of questions endorsed at a score of 3 , 4, or 5 on each of the three subscales. The three subscales of intrusion, avoidance, and arousal are determined by the respondent's answers on specific questions (intrusion: questions $2,3,6,10$, 13; avoidance: questions $1,5,7,9,12,14,17$; arousal: questions $4,8,11,15,16)$. If a respondent rating is at three (occasionally) or above for one or more items on the intrusion scale, three or more items on the avoidance scale, or two or more items on the arousal scale, then the respondent might be at a diagnostic level of secondary stress due to PTSD (Bride et al., 2007).

The STSS was pilot-tested by Bride and colleagues (2004) to provide reliability data for the three subscales (intrusion, avoidance, and arousal). The psychometric properties of the STSS include empirical data to substantiate the reliability of the entire questionnaire: a Cronbach's alpha coefficient value of .94 for the 17 items (2004). The alpha coefficient values for each of the subscales were reported by Bride et al. (2004): intrusion (.83), avoidance (.89), and arousal (.84).

Benuto, Yang, Ahrendt, and Cummings (2018) conducted a confirmatory factor analysis and found the three factors evidenced good fit for the three factors of intrusion, avoidance, and 
arousal. The STSS possesses adequate convergent validity, factorial validity, and "high levels of internal consistency" (Bride, 2007, p. 65) with significant correlations with similar constructs (Bride et al. 2004; Ting, Jacobson, Sanders, Bride, \& Harrington, 2005).

Criterion validity refers to whether the assessment is correlated with the established criteria being assessed (Salkind, 2017; Steele, Dunlavy, Stillman, \& Pape, 2011). The STSS was found to evidence adequate criterion validity (Ruel et al., 2016) for various helper populations (see Table 3-2), especially social workers responding to their perceptions about stress after working with clients (Bride, 2004). Bride et al. (2004) found that by examining the extent of trauma in the client population and the frequency that the helpers' work $(\mathrm{N}=287)$ addressed that trauma, the severity of STS symptoms, like depression, during the past week could be assessed with the 17 -item probe.

Table 3-2: Criterion Validity of STSS within the Context of Additional Results

\begin{tabular}{|c|c|c|c|c|c|}
\hline $\begin{array}{c}\text { Instrument } \\
\text { Study }\end{array}$ & $\begin{array}{l}\text { Language } \\
\text { and } \\
\text { occupation }\end{array}$ & $\begin{array}{c}\text { Sample } \\
\text { size }\end{array}$ & $\begin{array}{l}\text { Mean and } \\
\text { Standard } \\
\text { Deviation }\end{array}$ & $\begin{array}{c}\text { Reliability } \\
\text { (alpha) }\end{array}$ & Validity ${ }^{\mathbf{a}}$ \\
\hline $\begin{array}{l}\text { STSS } \\
\text { (Bride et al., } \\
\text { 2004). }\end{array}$ & $\begin{array}{l}\text { English } \\
\text { Social } \\
\text { workers }\end{array}$ & $\mathrm{N}=287$ & 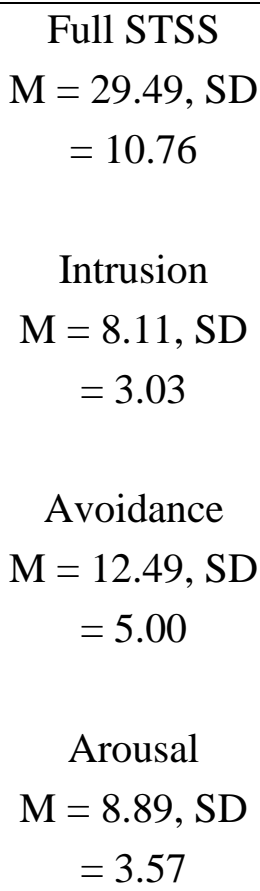 & $\begin{array}{l}\alpha=.80 \\
\alpha=.87 \\
\alpha=.83\end{array}$ & $\begin{array}{c}\text { Confirmatory Factor } \\
\text { Analysis }(\mathrm{CFA})^{\mathrm{c}} \\
\text { Intrusion/Avoidance } \\
\mathrm{r}=.87 \\
\text { Intrusion/Arousal } \\
\mathrm{r}=.94 \\
\text { Avoidance/Arousal } \\
\mathrm{r}=.97\end{array}$ \\
\hline
\end{tabular}




\begin{tabular}{|c|c|c|c|c|c|}
\hline $\begin{array}{l}\text { STSS } \\
\text { (Ting, } \\
\text { Jacobson, } \\
\text { Sanders, } \\
\text { Bride, \& } \\
\text { Harrington, } \\
\text { 2005) }\end{array}$ & $\begin{array}{l}\text { English } \\
\text { Social } \\
\text { workers }\end{array}$ & $\mathrm{N}=275$ & $\begin{array}{c}\text { Total } \\
\text { Intrusion } \\
\text { Avoidance and } \\
\text { Arousal }\end{array}$ & $\begin{array}{l}\alpha=.94 \\
\alpha=.79 \\
\alpha=.85 \\
\alpha=.87\end{array}$ & $\begin{array}{c}\mathrm{CFA}^{\mathrm{c}} \\
\text { Intrusion/Avoidance } \\
\mathrm{r}=.96 \\
\text { Intrusion/Arousal } \\
\mathrm{r}=.96 \\
\text { Avoidance/Arousal } \\
\mathrm{r}=1.0 \\
\text { (to determine if the } \\
\text { data fit the factor } \\
\text { model) }\end{array}$ \\
\hline $\begin{array}{l}\text { Secondary } \\
\text { Traumatic } \\
\text { Stress } \\
\text { Scale- } \\
\text { French } \\
\text { Version } \\
\text { (STSS-F; } \\
\text { Jacobs, } \\
\text { Charmillot, } \\
\text { Martin } \\
\text { Soelch, \& } \\
\text { Horsch, } \\
\text { 2019) }\end{array}$ & $\begin{array}{l}\text { French } \\
\text { Hospital } \\
\text { midwives }\end{array}$ & $\mathrm{N}=200$ & $\begin{array}{l}\text { Full STSS-F } \\
\begin{array}{c}\mathrm{M}=31.71, \mathrm{SD} \\
=10.09\end{array}\end{array}$ & $\alpha=0.92$ & $\begin{array}{l}\text { Two-factor model } \\
\text { Intrusion: } r=0.77 \\
\text { Avoidance-Arousal: } \\
\qquad r=0.95 \\
\text { Factor validity (+) } \\
\text { Concurrent validity } \\
\qquad+ \text { ) } \\
\text { Fit might only hold for } \\
\text { French version of } \\
\text { STSS which is two- } \\
\text { factor, not three factor } \\
\text { (Jacobs et al., 2019) }\end{array}$ \\
\hline $\begin{array}{l}\text { STSS } \\
\text { (Beckman, } \\
2015)\end{array}$ & $\begin{array}{c}\text { English } \\
\text { American } \\
\text { Red Cross } \\
\text { disaster } \\
\text { responders } \\
\text { and } \\
\text { disaster } \\
\text { mental } \\
\text { health } \\
\text { workers }\end{array}$ & $\mathrm{N}=92$ & $\begin{array}{c}\text { STSS total was } \\
\mathrm{M}=28.34 \\
\mathrm{SD}=9.36 . \\
(\mathrm{N}=81, \text { due to } \\
\text { incomplete } \\
\text { surveys })\end{array}$ & $\begin{array}{c}\text { STSS Total } \\
\alpha=.912 \\
\text { Avoidance } \\
\alpha=.822 \\
\text { Intrusion } \\
\alpha=.744 \\
\text { Arousal } \\
\alpha=.843\end{array}$ & $\begin{array}{c}\text { Narrative reporting } \\
\text { only: } \\
\text { "The STSS has also } \\
\text { demonstrated } \\
\text { convergent and } \\
\text { discriminant validity" } \\
\text { (p. 81). } \\
\text { Validity (n) }\end{array}$ \\
\hline STSS & English & $\mathrm{N}=121$ & $\begin{array}{l}\text { Study reported } \\
\text { correlation of }\end{array}$ & $\begin{array}{c}\text { STSS Total } \\
\mathrm{a}=.93\end{array}$ & $\begin{array}{l}\text { Narrative description: } \\
\text { "convergent, }\end{array}$ \\
\hline
\end{tabular}




\begin{tabular}{|c|c|c|c|c|c|}
\hline $\begin{array}{l}\text { (Badger, } \\
\text { Royse, \& } \\
\text { Craig, 2008) }\end{array}$ & $\begin{array}{l}\text { Hospital } \\
\text { social } \\
\text { workers }\end{array}$ & & $\begin{array}{c}\text { STSS with } \\
\text { other measures }\end{array}$ & & $\begin{array}{c}\text { discriminant, and } \\
\text { factorial validity were } \\
\text { also tested, with } \\
\text { excellent results" } \\
\text { Validity (n) }\end{array}$ \\
\hline $\begin{array}{l}\text { STSS } \\
\text { (Benuto, } \\
\text { Yang, } \\
\text { Ahrendt, \& } \\
\text { Cummings, } \\
\text { 2018) }\end{array}$ & $\begin{array}{c}\text { English } \\
\text { Victim } \\
\text { advocates }\end{array}$ & $\mathrm{N}=135$ & $\begin{array}{c}\text { Study reported } \\
\text { STSS } M \text { and } \\
S D \text { by } \\
\text { question, not } \\
\text { by total }\end{array}$ & $\begin{array}{c}\text { STSS Total } \\
\alpha=.93 \\
\text { Intrusion } \\
\alpha=.80 . \\
\text { Avoidance } \\
\alpha=.85 \\
\text { Arousal } \\
\alpha=.79\end{array}$ & $\begin{array}{c}\text { CFI }=0.931, \\
\text { indicating a } 93.1 \% \\
\text { improvement of the } \\
\text { current model (Benuto } \\
\text { et al., 2018) compared } \\
\text { with a baseline model } \\
\text { of STSS (Bride et al., } \\
\text { 2004). }\end{array}$ \\
\hline
\end{tabular}

${ }^{a}$ Validity: Plus (+) indicates validity is supported; Letter n (n) indicates validity is reported narratively. ${ }^{\mathrm{b}} \mathrm{A}$ twofactor model with pooled avoidance-arousal scale yielded acceptable fit. ${ }^{c}$ Data could provide support for STS as unidimensional and not three distinct subscales, per Beckman (2015).

Convergent validity was supported in extent $(M=3.19, S D=.87)$, frequency $(M=3.49$, $S D=.93)$, and severity $(M=1.74, S D=.79)$. Bride et al. (2004) noted that the STSS is useful for other helping populations, including educators. Because I am exploring STS in public K-12 teachers in three areas of opioid impact, I utilized a definition from Motta (2012) for the STS construct: the "transfer and acquisition of negative affective and dysfunctional cognitive states due to prolonged and extended contact with others, such as family members, who have been traumatized" (p. 257). This way, teachers are similar to social workers in that teachers have prolonged daily contact with children in schools (Schepers, 2017). I took care not to change or revise any of the original STSS questions except for revising the wording to pertain to educators (i.e., change clinician to teacher, change client to student). 
Open-ended questions. The following open-ended questions were added to the survey to allow respondents to offer feedback in their own words:

- Open-ended question 1: What do you find difficult about supporting students who experience significant adversity and trauma? How is it difficult?

- Open-ended question 2: What do you find positive or rewarding about supporting students who experience significant adversity and trauma? How is it positive for you?

- Open-ended question 3: How do you cope with the stress of supporting students who experience significant adversity and trauma? What works best for you?

- Open-ended question 4: What else would you like to say about your experiences supporting children who experience significant adversity and trauma?

Ruel et al. (2016) noted that open-ended questions could be difficult to quantify and, therefore, might not be as useful in statistical analysis. For this study, the questions invited the respondents to share but did not require any answer. This way, the questions served a dual-purpose of an opportunity to reflect about stress, which can be part of a stress-relief strategy (Hayes, 2006) and as a means of collecting additional and illustrative information. For this study, the open-ended questions are used illustratively to illuminate the findings in Chapter Five.

\subsection{Data Collection Procedures}

Institutional Review Board (IRB) approval was received from Chapman University before beginning data collection. An online data collection procedure was conducted through Qualtrics, a concierge-database service that maintains data protection and data security (Qualtrics, 2014).

The online instrument (see Appendix B) included a brief description of the research which was presented to the sample:

The purpose of this survey is to explore the experience of teachers who work with children who have experienced trauma. People who support others, like nurses in an 
emergency room or social workers after a natural disaster, can sometimes be affected by a type of stress called secondary traumatic stress. Secondary traumatic stress is defined as behavior and emotions that result from supporting others who have experienced trauma. This survey will help gather information about teachers, focusing on secondary traumatic stress. Your participation in this research is voluntary. You may choose not to participate. If you decide to participate in this research survey, you may withdraw at any time. If you decide not to participate in this study or if you withdraw from participating at any time, you will not be penalized. The procedure involves completing the online survey, which will take approximately 20-30 minutes. Your responses will all be anonymous and confidential. At no time is identifying information collected for the survey, like your name, email address, or IP address.

A soft launch of the questionnaire was delivered via email through Qualtrics to a sample population of K-12 public school teachers $(\mathrm{n}=150)$ to assess whether the survey was functioning appropriately and returning meaningful results to all question probes. An explanation of the study was only provided after the respondent consented to the study. For the soft launch, participants were provided with informed consent forms, were assured about the confidential nature of the survey, and were advised that they could opt-out at any given time if they were feeling any distress.

After evaluating the results of the soft launch, several questionnaire items were revised. The zip code of the teacher's school was amended to include the appropriate numerical responses. Additionally, a question was added as a logic shield question ("Which of the following best describes your profession?"). If the participants did not select "teacher," they were deselected for the survey. This logic question was added as an extra assurance that 
participants were reading the survey and responding to survey questions. These adjustments improved the consistency of the survey: survey zip codes were aligned with national postal zip codes, and only self-identified teachers were respondents to the survey.

Two criteria determined the goal of 450 completed surveys. First, there are three opioid areas (low, medium, and high). Each area required an equal number of respondents to run statistical assessments. Second, care was taken to determine sample size in each area for the effect to be statistically significant, if this is shown in the analysis (Ruel et al., 2016). The sample size of $n=150$ from each of the three zones corresponds with the recommendation for a sample size of at least 100 for a meaningful result (Bisits, 2014).

Qualtrics sent the instrument out in October 2019. This data collection time was chosen intentionally. By distributing the surveys in October, I was able to assure that teachers had started school (after September) and that the survey was completed before the holidays in November/December. When 450 completed surveys had been returned, the survey closed. Upon audit, it was discovered that the 450 surveys did not conform to the research stipulations of the study. More surveys were needed in each of the three areas (low, medium, and high). Although there were 450 surveys, states that were outside of the 26 -state list had inadvertently been included. Because of this, Qualtrics re-opened the survey and redistributed it until 150 completed surveys were returned in the designated areas. After this second distribution, the proper number for each area were returned so that the study goal of 150 completed questionnaires from each of the three areas was met.

Completing the survey took less than ten minutes, on average, as shown by the time report provided by Qualtrics and verified by the researcher by assessing the data reflecting time spent on each survey. No identifiable data were collected from any participant, and participation 
was voluntary. Additionally, teachers could choose not to complete the survey once they had begun it or at any time during the process. Participants received a small compensation of \$20 each, administered by Qualtrics and supplied by the researcher, in return for their participation. There was no penalty if respondents chose not to participate. At the end of the survey, there was supportive information supplied by the researcher. If teachers experienced any stress or illeffects from the survey or if they wanted more information about secondary stress or impact of trauma, national referrals were provided as a separate link.

When the survey closed for the second time, the Qualtrics program manager forwarded the results of the survey on an Excel spreadsheet. On the 450 questionnaires, every quantitative question had been answered; there were no missing data. Although web-based surveys can have a response rate that fails to meet the higher rate of a mail-in survey (Couper, 2000), working with Qualtrics, soft-launching the survey, collaborating with the project manager and offering compensation mitigated those circumstances to allow for the $100 \%$ response completion rate. Also, the questionnaire results were certified by Qualtrics and rated, using the Qualtrics rubric for quality rating, as the highest level of quality. The highest level means that participants did not skip questions, took time to answer, and did not select all of one choice for the multiple-choice questions. Respondents appeared to give answers that performed within the logic frame necessary for Qualtrics’ highest rating (of five scaled scores) for quality assessment.

After receiving the final Excel spreadsheet from Qualtrics, I separated the data into the three areas (low, medium, and high) for analysis using a zip code sort with Excel. The nonrepresentative states were purged, and the final count was completed in each of the three areas to ensure $\mathrm{n}=150$ for each area. The qualitative responses were separated from the quantitative data 
and were similarly sorted into low, medium, and high opioid impacted zones. The data and data analysis files were stored on a password-protected computer in the home of the researcher.

\subsection{Statistical Analyses of Study Data}

The quantitative data were downloaded from the Qualtrics Excel file. They were recoded into an Excel file for data analysis. The data were coded with variable labels and corresponding values and then checked for accuracy. The overall score and the three scales from the STSS were statistically computed. The ACE data with the 14 trauma categories were entered as checked (1) or not checked (0). Demographic profile data were incorporated into the data file with nominal or ordinal coding. For example, for the category of Marital Status, the following numerical values were assigned: divorced (1); in a committed partnership (2); married (3); other (4); single (5); and widowed (6). The key to the numerical codes is listed in Appendix D.

Before executing the statistical analyses, descriptive statistics were conducted on the demographic factors reported by the sample. Also, each of the 17 items on the SSTS and 14 items on the ACE was presented in terms of responses by the sample of participants. The means and standard deviations of the overall SSTS score, and with the three subscale scores on intrusion, avoidance, and arousal were presented as descriptive data. This descriptive data addressed the first research question: RQ1: What are $K-12$ teachers' self-reported levels of secondary traumatic stress?

The second and third research questions were examined by the use of a nonparametric procedure, the Spearman's rank-order correlation coefficient (i.e., Spearman's Rho), which is used to examine relationships among variables. This statistic was used to measure the strength and the direction of the association of the variables after ranking them. The second research question is the following: RQ2: Is there a relationship between the teachers' self-reported levels 
of secondary traumatic stress when teachers are from states of high-opioid impact, states of medium-opioid impact, and states of low-opioid impact? Spearman's Rho is used when data are ranked as a form of ordinal data (Urdan, 2017). Because the three opioid zones are ranked using mortality rate and annual percent of change to determine opioid-zone of low, medium, and high, they are already ordinal. The STSS total scores and subscale scores were ranked, and a Spearman's rank-order correlation was performed. In this way, it was possible to calculate the correlation between two variables using the ranked data of opioid zone and the ranked data of STSS scores.

The third research question, which is detailed in four sub-questions below, was also correlational, necessitating the same procedure for the Spearman's rank correlation:

- RQ3: Is there a relationship between self-reported levels of secondary traumatic stress and characteristics of teachers?

The demographic characteristics of age, gender, race, and relationship status (which was questioned on the survey as marital status) were ranked as potential correlates of secondary traumatic stress. Again, Spearman's Rho was used to calculate the correlation between the ranked ordinal data of low, medium, and high opioid zone with the ranked demographic data to see whether a teacher's stress level in a particular opioid zone was related to their demographic characteristic. This analysis addressed the four research sub-questions:

- RQ3a: Is there a relationship between self-reported levels of secondary traumatic stress and the age of teachers?

- RQ3b: Is there a relationship between self-reported levels of secondary traumatic stress and gender?

- RQ3c: Is there a relationship between self-reported levels of secondary traumatic stress and racelethnicity? 
- RQ3d: Is there a relationship between self-reported levels of secondary traumatic stress and relationship status?

Even though the demographic variables were not necessarily ranked at the time of collection, like gender, Spearman's Rho can be used to "test the association between one ranked variable and one measurement variable" (McDonald, 2014, p. 210). When evaluating the data with Spearman's Rho, all the data were subsequently ranked.

The qualitative questions in the survey were thematically assessed and analyzed in light of the goals of the research questions (Braun \& Clark, 2006). The qualitative questions were optional, so they are used illustratively to illuminate the quantitative data.

\subsection{Conclusion}

The methods and procedures for this study were presented in this chapter. By considering the research questions, the research design, the sampling strategy, the instrumentation, data collection procedures, and the statistical analyses, the purpose is more clearly elucidated. Each section of the survey is designed to focus our understanding of the research questions.

Teachers are first asked about themselves in ways that will reveal demographic information without revealing identifying information. This demographic information is useful in research to help explore any variables or traits that might be associated with increased or decreased risk for stress. For example, if the study reveals that male teachers or younger teachers are at a high level of risk, further research could direct policy to support these populations. Additionally, the investigation of K-12 teachers' self-reported level of secondary traumatic stress (STS) is built on the assumption that there are students in K-12 classes who have experienced life circumstances that result in trauma responses. Teachers' answers to the PHL-ACEs questions can guide our growing understanding of the added areas of concern that teachers face. As respondents answer the STSS, the information has the potential to be added to an increasing 
body of national data on teacher stress and secondary traumatic stress of helpers. Finally, the open-ended questions are not only for the benefit of the researcher or this study; in past studies of teacher STS, the opportunity to express emotions is limited. The open-ended questions offer an opportunity to reflect on challenges, strengths, and coping mechanisms involved in teacher secondary traumatic stress. 


\section{Chapter Four: Results}

\subsection{Results}

The purpose of this study was to investigate if K-12 teachers' self-reported level of secondary traumatic stress (STS) related to opioid mortality level in their communities and if their levels of STS were correlated with any individual characteristics. This chapter first reviews the measure and plan of analysis. Next, the results are presented: these include descriptive and correlational analyses for data collected from 450 teachers regarding their identification of student trauma and their self-report of STS. Descriptive statistics are presented first, followed by correlations.

\subsubsection{Measures and Plan of Analysis}

This study used descriptive statistics, a correlational analysis, and a measure of internal consistency, which will be described first. Next, the analysis procedures and findings are discussed.

Descriptive statistics. Several different measures of descriptive statistics are utilized to understand the sample. These include the following:

- Frequency statistics: frequency (raw counts) and relative frequency (percentages)

- Location statistics: mean (arithmetic average, as a measure of central tendency)

- Dispersion statistics, including the range (minimum and maximum values) and standard deviation (average deviations from the mean)

Dispersion statistics, like standard deviation, give additional information about the variability of the data (Larson, 2006). Because the sample was not collected randomly, it is important to remember that these are descriptors and not inferences about the population of teachers nationally (Urdan, 2017). 
Correlational analysis. Correlational measures can show whether variables are related to one another (Urdan, 2017), so they are useful for examining relationships. Spearman's rank correlation coefficient (Spearman's Rho) is a nonparametric correlational analysis used to measure the strength of a relationship between paired data, with the assumption that as one variable increases, the other variable either increases or decreases (McDonald, 2014). Assumptions are underlying parametric statistical procedures, which include, but are not limited to, the distribution shape (normal distribution) in the population and the two parameters (means and standard deviations) of that assumed normal distribution (McDonald, 2014). These assumptions inform considerations about the parameters of the population's distribution, and the sample or data would be drawn from this population. Conversely, nonparametric statistical procedures, like Spearman's Rho, rely on no or few assumptions about the shape or parameters of the population distribution from which the sample or data were drawn. While the sample may be normally distributed, the use of Spearman's Rho allows for a correlational measure between variables without the assumption of normal distribution.

The values for Spearman's Rho range from -1 to +1 . A value of -1 would indicate a negative correlation between the data sets, called the ranks; a value of +1 would indicate a positive correlation; a value of 0 would indicate no correlation. This correlation is also called a measure of association. There are no conventions in the literature that are fixed as to what determines a weak association or strong association (Ruel et al., 2016), but there are commonalities in the literature. Akoglu (2018) combined three scales from various studies in three different disciplines to interpret the range of Spearman's Rho values from -1 to +1 (see Figure 4-1). 
Figure 4-1: Akoglu's (2018) Spearman's Rho Correlation Coefficient Interpretation Comparison Interpretation of the Pearson's and Spearman's correlation coefficients.

\begin{tabular}{lllll}
\hline $\begin{array}{l}\text { Correlation } \\
\text { Coefficient }\end{array}$ & & $\begin{array}{l}\text { Dancey \& Reidy } \\
\text { (Psychology) }\end{array}$ & $\begin{array}{l}\text { Quinnipiac } \\
\text { University } \\
\text { (Politics) }\end{array}$ & $\begin{array}{l}\text { Chan YH } \\
\text { (Medicine) }\end{array}$ \\
\hline+1 & -1 & Perfect & Perfect & Perfect \\
+0.9 & -0.9 & Strong & Very Strong & Very Strong \\
+0.8 & -0.8 & Strong & Very Strong & Very Strong \\
+0.7 & -0.7 & Strong & Very Strong & Moderate \\
+0.6 & -0.6 & Moderate & Strong & Moderate \\
+0.5 & -0.5 & Moderate & Strong & Fair \\
+0.4 & -0.4 & Moderate & Strong & Fair \\
+0.3 & -0.3 & Weak & Moderate & Fair \\
+0.2 & -0.2 & Weak & Weak & Poor \\
+0.1 & -0.1 & Weak & Negligible & Poor \\
0 & 0 & Zero & None & None \\
\hline
\end{tabular}

Note. Table representing a comparison, across disciplines, of three various interpretations for Spearman's Rho values. From "User's guide to correlation coefficients," by H. Akoglu, 2018, Turkish Journal of Emergency Medicine, 18(3), p. 92. Copyright 2018 by Creative Commons Attribution-NonCommercial-NoDerivatives 4.0 International Public License. Permission granted by author. See Appendix E. The figure, in its original article, can be found here: https://www.sciencedirect.com/science/article/pii/S2452247318302164

Measure of internal consistency. Cronbach's alpha $(\alpha)$, also called coefficient alpha, is a measure of internal consistency or reliability (Cronbach, 1951), which was calculated to determine the measure of internal consistency of the STSS, to examine if the questions were assessing for the same construct-secondary traumatic stress. Cronbach (1951) described alpha as intending to demonstrate whether the test item collection yields interpretations that are reliable or consistent about respondents. The measure of internal consistency reliability is used to determine whether the test is assessing the same construct or dimension (Salkind, 2017). Cronbach's alpha is used most commonly for reliability analysis that examines internal consistency (Bonett \& Wright, 2015; Urdan, 2017). Although there are a range of qualitative descriptors for Cronbach's alpha in the research literature (Taber, 2017), George and Mallery (2003) offered an interpretation scale for alpha (see Table 4-1). The table provides an acceptable qualitative interpretation scale used widely: "_> $.9-$ Excellent, $\_>.8-$ Good, $_{-}>.7-$ 


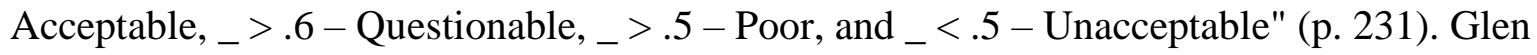
(2014) added that a large alpha might indicate redundancy in the test items.

Table 4-1: Cronbach's Alpha Scale Interpretation

\begin{tabular}{|c|c|}
\hline $\begin{array}{c}\text { Cronbach's alpha } \\
(\boldsymbol{\alpha})\end{array}$ & $\begin{array}{c}\text { Rating of Internal } \\
\text { Consistency }\end{array}$ \\
\hline$\alpha>.095$ & $\begin{array}{c}\text { Possible indicator of } \\
\text { redundant questions }\end{array}$ \\
\hline$\alpha \leq 0.9($ or $\alpha \leq 0.95)$ & Excellent \\
\hline $0.9<\alpha \leq 0.8$ & Good \\
\hline $0.8<\alpha \leq 0.7$ & Acceptable \\
\hline $0.7<\alpha \leq 0.6$ & Questionable \\
\hline $0.6<\alpha \leq 0.5$ & Poor \\
\hline $0.5<\alpha$ & Unacceptable \\
\hline
\end{tabular}

Note. Table values and interpretation determined by George and Mallery (2003) and Glen (2014). These are considered industry guidelines.

Plan of analysis. After the quantitative data were downloaded from the Qualtrics Excel file and recoded for data analysis, the overall STSS score and the three scales were statistically computed. Descriptive statistics were conducted on the demographic factors reported by the sample. Also, each of the 17 items on the SSTS and 14 items on the ACE was presented in terms of responses by the sample of participants. The means and standard deviations of the overall SSTS score, and with the three subscale scores on intrusion, avoidance, and arousal were presented as descriptive data addressing the first research question: $R Q 1$ : What are $K-12$ teachers' self-reported levels of secondary traumatic stress?

The second and third research questions were examined using Spearman's rank-order correlation coefficient (i.e., Spearman's Rho). This nonparametric procedure was used to measure the strength and the direction of the association of the variables after ranking them. The second research question is the following: RQ2: Is there a relationship between the teachers' self-reported levels of secondary traumatic stress when teachers are from states of high-opioid 
impact, states of medium-opioid impact, and states of low-opioid impact? It was possible to calculate the correlation between two variables using the ranked data of opioid zone and the ranked data of STSS scores.

The third research question, also correlational, necessitated the use of Spearman's rank correlation: RQ3: Is there a relationship between self-reported levels of secondary traumatic stress and characteristics of teachers? The demographic characteristics of age, gender, race, and relationship status were ranked as potential correlates of secondary traumatic stress. The results of Spearman's Rho addressed the four research sub-questions: RQ3a: Is there a relationship between self-reported levels of secondary traumatic stress and the age of teachers? RQ3b: Is there a relationship between self-reported levels of secondary traumatic stress and gender? RQ3c: Is there a relationship between self-reported levels of secondary traumatic stress and race/ethnicity? RQ3d: Is there a relationship between self-reported levels of secondary traumatic stress and relationship status? Even though the demographic variables were not necessarily ranked at the time of collection, like gender, Spearman's Rho can be used to "test the association between one ranked variable and one measurement variable" (McDonald, 2014, p. 210).

Cronbach's alpha was calculated to measure the internal consistency of the STSS with the 450 teachers, to see if the scale measured the construct of secondary stress with this sample. The STSS means, standard deviation, and sample total $(\mathrm{N}=450)$ were used in the calculation. Because alpha is reported on a scale between $0-1$, the score can then be interpreted in light of industry standards to ascertain if the STSS has a suitable level of internal consistency for this study. 
In addition to the quantitative analysis, the qualitative features of the survey were analyzed in light of the goals of the research questions by thematic analysis (Braun \& Clark, 2006). Because the qualitative questions were optional, they are used to illuminate the finding of the quantitative analyses through illustrative examples.

\subsection{Sample Characteristics}

For this section, the results of descriptive statistics will be used for two major purposes. First, teacher demographics are more clearly understood by examining age, gender, race/ethnicity, relationship status, and primary teaching assignment. Second, the comparison between the sample population demographics and national teacher demographics are explored. Measures used are frequency statistics, including frequency (raw counts) and relative frequency (percentages); location statistics, including the mean (arithmetic average, as a measure of central tendency); and dispersion statistics, including the range (minimum and maximum values) and standard deviation (average deviations from the mean). Dispersion statistics, like standard deviation, give additional information about the variability of the data (Larson, 2006). Because the focus of this study is a comparison of the three opioid epidemic zones, when applicable, the descriptors will be reported for the different zones. The demographic descriptors of age, gender, race/ethnicity, relationship status (which was queried as married, single, etc.), primary teaching assignment, and opioid epidemic zone are the independent variables for the study.

The sample is composed of $450 \mathrm{~K}-12$ teachers. Descriptive statistics were conducted on demographic and research variables to profile the sample. These descriptions divide into two groups: 1) teacher descriptions identify the qualities of the survey participants, and 2) adverse experiences descriptions identify how participants describe the trauma issues experienced by students. Table 4-2 contains the identifying attributes of the survey participants for the whole 
sample population $(\mathrm{N}=450)$. In each of the sections following the table, these variables from

Table 4-2 will be further explored for each of the three opioid zones $(n=150)$ : gender, age,

relationship status, race/ethnicity primary teaching assignment, and years of experience teaching.

Table 4-2: Demographic and Geographic Characteristics of K-12 Teachers

\begin{tabular}{|c|c|c|}
\hline Characteristic & $n$ & $\%$ \\
\hline \multicolumn{3}{|l|}{ Gender } \\
\hline Female & 319 & 70.89 \\
\hline Male & 124 & 27.56 \\
\hline Non-binary & 5 & 1.11 \\
\hline Other & 2 & 0.44 \\
\hline \multicolumn{3}{|l|}{ Age range } \\
\hline 20-30 years-old & 128 & 28.44 \\
\hline $31-40$ & 175 & 38.89 \\
\hline $41-50$ & 93 & 20.67 \\
\hline $51-60$ & 30 & 6.67 \\
\hline Over 60 & 24 & 5.33 \\
\hline \multicolumn{3}{|l|}{ Marital Status } \\
\hline Divorced & 29 & 6.44 \\
\hline Partnership & 57 & 12.67 \\
\hline Married & 236 & 52.44 \\
\hline Single & 119 & 26.44 \\
\hline Widowed & 7 & 1.56 \\
\hline Other & 2 & 0.44 \\
\hline \multicolumn{3}{|l|}{ Race/Ethnic Identity } \\
\hline $\begin{array}{l}\text { American Indian or } \\
\text { Alaskan Native (AI/AN) }\end{array}$ & 5 & 1.11 \\
\hline Asian & 21 & 4.67 \\
\hline Black or African American & 69 & 15.33 \\
\hline $\begin{array}{l}\text { Combination of two or } \\
\text { more races }\end{array}$ & 12 & 2.67 \\
\hline Hispanic/Latino/a & 54 & 12.00 \\
\hline $\begin{array}{l}\text { Native Hawaiian or other } \\
\text { Pacific Islander }\end{array}$ & 1 & 0.22 \\
\hline White & 285 & 63.33 \\
\hline Other & 2 & 0.44 \\
\hline
\end{tabular}




\begin{tabular}{|c|c|c|}
\hline Prefer not to identify & 1 & 0.22 \\
\hline Primary teaching assignment $^{\text {a }}$ & & \\
\hline Alternative school $^{\mathrm{b}}$ & 3 & 0.67 \\
\hline Blended class $^{\mathrm{c}}$ & 16 & 3.56 \\
\hline Elementary school $^{\mathrm{a}}$ & 155 & 34.44 \\
\hline Middle school & 90 & 20.00 \\
\hline High School & 124 & 27.56 \\
\hline Multiple grades & 23 & 5.11 \\
\hline Special education & 33 & 7.33 \\
\hline Other $^{\text {Prefer not to identify }}$ & 3 & 0.67 \\
\hline Geographic identifier $^{\mathrm{d}}$ & 3 & 0.67 \\
\hline Low opioid zone $^{\mathrm{d}}$ & 150 & \\
\hline Medium opioid zone & 150 & 33.33 \\
\hline High opioid zone & 150 & 33.33 \\
\hline
\end{tabular}

${ }^{a}$ Reflects the diversity of teaching placements in the different states. ${ }^{\mathrm{b}}$ Alternative education is typically continuation, credit recovery, etc., as identified by state credentialing board. ${ }^{\mathrm{c}}$ Blended often means to teach a variety of classes/grades from K-8 or K-12. This was the definition provided on the survey for teachers to self-select. ${ }^{\mathrm{d}}$ Refers to the epidemic zone, as defined by the mortality indicators, as described in Chapter 3.

\subsubsection{Teacher Gender}

Table 4-3 shows the number and percent of teachers by gender in each of the three opioid zones. Seventy-one percent or 319 of the teachers were female, while $28 \%$ or 125 were male in the total sample. A few teachers selected non-binary as their gender identity. There was little variation between the three zones.

Table 4-3: Teacher Gender

\begin{tabular}{|l|rr|rr|rr|rl|}
\hline \multicolumn{1}{|c|}{ Group } & N & $\mathbf{( \% )}$ & \multicolumn{6}{|c|}{ Opioid Zone } \\
\hline & & & \multicolumn{2}{|c|}{ Low } & \multicolumn{1}{c|}{ Medium } & \multicolumn{2}{c|}{ High } \\
\hline & & & $\mathbf{N}$ & $\mathbf{\%}$ & $\mathbf{N}$ & $\mathbf{\%}$ & $\mathbf{N}$ & $\mathbf{\%}$ \\
\hline Female & 319 & $71 \%$ & 103 & $69 \%$ & 111 & $74 \%$ & 105 & $70 \%$ \\
\hline Male & 124 & $28 \%$ & 45 & $30 \%$ & 36 & $24 \%$ & 43 & $29 \%$ \\
\hline Non-Binary & 5 & $1 \%$ & 2 & $1 \%$ & 3 & $2 \%$ & 0 & $0 \%$ \\
\hline Other & 2 & $0 \%$ & 0 & $0 \%$ & 0 & $0 \%$ & 2 & $1 \%$ \\
\hline Total & 450 & $100 \%$ & 150 & $100 \%$ & 150 & $100 \%$ & 150 & $100 \%$ \\
\hline
\end{tabular}




\subsubsection{Teacher Age}

For the total sample, the most frequently reported age segment for teachers was 31-40, with over one-third of the respondents indicating they fell in this age range (see Table 4-3). The second largest was 20-30 (28\%) and then 41-50 (21\%). A much smaller number reported being over 51 years of age $(12 \%)$. The differences between the opioid zones for ages were minimal.

Table 4-4: Teacher Age Segments

\begin{tabular}{|l|lr|rr|rr|rc|}
\hline \multicolumn{1}{|c|}{ Group } & N & $\mathbf{( \% )}$ & \multicolumn{6}{|c|}{ Opioid Zone } \\
\hline & & & \multicolumn{2}{|c|}{ Low } & \multicolumn{2}{c|}{ Medium } & \multicolumn{2}{c|}{ High } \\
\hline & & & N & \% & N & \% & N & \% \\
\hline $\mathbf{2 0 - 3 0}$ & 128 & $28 \%$ & 49 & $33 \%$ & 40 & $27 \%$ & 39 & $26 \%$ \\
\hline $\mathbf{3 1 - 4 0}$ & 175 & $39 \%$ & 59 & $39 \%$ & 52 & $35 \%$ & 64 & $43 \%$ \\
\hline $\mathbf{4 1 - 5 0}$ & 93 & $21 \%$ & 29 & $19 \%$ & 37 & $25 \%$ & 27 & $18 \%$ \\
\hline $\mathbf{5 1 - 6 0}$ & 30 & $7 \%$ & 5 & $3 \%$ & 15 & $10 \%$ & 10 & $7 \%$ \\
\hline Over 60 & 24 & $5 \%$ & 8 & $5 \%$ & 6 & $4 \%$ & 10 & $7 \%$ \\
\hline Total & 450 & $100 \%$ & 150 & $100 \%$ & 150 & $100 \%$ & 150 & $100 \%$ \\
\hline
\end{tabular}

\subsubsection{Teacher Relationship Status}

Over half of the teachers in the total sample were married (52\%) and a quarter (26\%) were single (see Table 4-5). Approximately $12 \%$ were in partnerships, divorced (6\%), or widowed (2\%). The marital status percentages were similar in the low and high zones. The medium zone had fewer single teachers at $19 \%$ (compared to $29 \%$ and $31 \%$ of the low and high zones). 
Table 4-5: Teacher Relationship/Marital Status

\begin{tabular}{|l|cc|cc|cc|cc|}
\hline \multicolumn{1}{|c|}{ Group } & \multicolumn{2}{|c|}{ N (\%) } & \multicolumn{6}{c|}{ Opioid Zone } \\
\hline & & & \multicolumn{2}{c|}{ Low } & \multicolumn{2}{c|}{ Medium } & \multicolumn{2}{c|}{ High } \\
\hline & & & $\mathbf{N}$ & $\mathbf{\%}$ & $\mathbf{N}$ & $\mathbf{\%}$ & $\mathbf{N}$ & $\mathbf{\%}$ \\
\hline Single & 119 & $(26 \%)$ & 43 & $29 \%$ & 29 & $19 \%$ & 47 & $31 \%$ \\
\hline Partnership & 57 & $(12 \%)$ & 20 & $13 \%$ & 18 & $12 \%$ & 19 & $13 \%$ \\
\hline Married & 236 & $(52 \%)$ & 78 & $52 \%$ & 86 & $57 \%$ & 72 & $48 \%$ \\
\hline Divorced & 29 & $(6 \%)$ & 9 & $6 \%$ & 11 & $7 \%$ & 9 & $6 \%$ \\
\hline Widowed & 7 & $(3 \%)$ & 0 & $0 \%$ & 5 & $3 \%$ & 2 & $1 \%$ \\
\hline Other & 2 & $(1 \%)$ & 0 & $0 \%$ & 1 & $0 \%$ & 1 & $0 \%$ \\
\hline Total & 450 & $(100 \%)$ & 150 & $100 \%$ & 150 & $100 \%$ & 150 & $100 \%$ \\
\hline
\end{tabular}

\subsubsection{Teacher Race/Ethnicity}

Race/ethnicity for this study is reported in a manner that is consistent with the method used by the U.S. Department of Education (Hussar et al., 2020) and compatible with APA standards for reducing bias (American Psychological Association, 2010). Because Hispanic origin is an ethnicity rather than a race, this category is reported as race/ethnicity to be both inclusive and consistent with national statistic reporting. The total sample was mostly White (63\%). Fifteen percent were Black, $12 \%$ were Hispanic, $5 \%$ were Asian (5\%), and 5\% were another race or ethnicity (5\%). In comparing the three opioid zones (see Table 4-6), there was ethnic diversity in the low opioid zone. The low zone sample was $48 \%$ White, compared to the medium zone (72\%) and high zone (70\%). The low zone also had a higher prevalence of Hispanic teachers, at 24\%, compared to $6 \%$ each for the medium and high zones.

Table 4-6: Teacher Race or Ethnicity

\begin{tabular}{|l|cc|cc|cc|cc|}
\hline Group & \multicolumn{1}{|c|}{$\mathbf{N}$} & $\mathbf{\%}$ & \multicolumn{6}{|c|}{ Opioid Zone } \\
\hline & & & \multicolumn{2}{|c|}{ Low } & \multicolumn{2}{|c|}{ Medium } & \multicolumn{2}{|c|}{ High } \\
\hline & & & $\mathbf{N}$ & $\mathbf{\%}$ & $\mathbf{N}$ & $\mathbf{\%}$ & $\mathbf{N}$ & $\%$ \\
\hline Asian & 21 & $5 \%$ & 14 & $9 \%$ & 3 & $2 \%$ & 4 & $3 \%$ \\
\hline Black & 69 & $15 \%$ & 21 & $14 \%$ & 22 & $15 \%$ & 26 & $17 \%$ \\
\hline Hispanic & 54 & $12 \%$ & 36 & $24 \%$ & 9 & $6 \%$ & 9 & $6 \%$ \\
\hline
\end{tabular}




\begin{tabular}{|l|cc|cc|cc|cc|}
\hline White & 285 & $63 \%$ & 72 & $48 \%$ & 108 & $72 \%$ & 105 & $70 \%$ \\
\hline Other & 20 & $5 \%$ & 7 & $5 \%$ & 7 & $5 \%$ & 6 & $4 \%$ \\
\hline Refused & 1 & $0 \%$ & 0 & $0 \%$ & 1 & $0 \%$ & 0 & $0 \%$ \\
\hline Total & 450 & $100 \%$ & 150 & $100 \%$ & 150 & $100 \%$ & 150 & $100 \%$ \\
\hline
\end{tabular}

\subsubsection{Teacher Primary Teaching Assignment}

A large segment of the teachers in the total sample was assigned to the elementary school setting (40\%). Almost equal percentages were at the middle (22\%) or high school (28\%) levels. There were small percentages of special education, blended or alternative education levels (see Table 4-7). In the three opioid zones, this pattern was repeated in the low and medium opioid zones but diverged slightly in the high zone. The teachers in the high opioid zone were more evenly spread between their teaching assignments.

Table 4-7: Primary Teaching Assignment

\begin{tabular}{|l|cc|cc|cc|cc|}
\hline \multicolumn{1}{|c|}{ Group } & N & $\mathbf{( \% )}$ & \multicolumn{6}{c|}{ Opioid Zone } \\
\hline & & & \multicolumn{2}{|c|}{ Low } & \multicolumn{2}{c|}{ Medium } & \multicolumn{2}{c|}{ High } \\
\hline & & & $\mathbf{N}$ & $\mathbf{\%}$ & $\mathbf{N}$ & $\mathbf{\%}$ & $\mathbf{N}$ & $\mathbf{\%}$ \\
\hline Elementary & 155 & $34 \%$ & 56 & $37 \%$ & 57 & $38 \%$ & 42 & $28 \%$ \\
\hline High school & 124 & $28 \%$ & 45 & $30 \%$ & 44 & $29 \%$ & 35 & $23 \%$ \\
\hline Middle & 90 & $20 \%$ & 25 & $17 \%$ & 29 & $19 \%$ & 36 & $24 \%$ \\
\hline Special Ed. & 33 & $7 \%$ & 8 & $5 \%$ & 7 & $5 \%$ & 18 & $12 \%$ \\
\hline Multiple & 23 & $5 \%$ & 6 & $4 \%$ & 10 & $7 \%$ & 7 & $5 \%$ \\
\hline Blended & 16 & $4 \%$ & 6 & $4 \%$ & 3 & $2 \%$ & 7 & $5 \%$ \\
\hline Alternative Ed & 3 & $1 \%$ & 0 & $0 \%$ & 0 & $0 \%$ & 3 & $2 \%$ \\
\hline Refused & 3 & $1 \%$ & 3 & $2 \%$ & 0 & $0 \%$ & 0 & $0 \%$ \\
\hline System & 3 & $1 \%$ & 1 & $1 \%$ & 0 & $0 \%$ & 2 & $1 \%$ \\
\hline Total & 450 & $100 \%$ & 150 & $100 \%$ & 150 & $100 \%$ & 150 & $100 \%$ \\
\hline
\end{tabular}

\subsubsection{Teacher Years of Experience}

In terms of the number of years teaching, the average for the 450 teachers was nine years $(S D=7)$. Table $4-8$ shows the mean for the years of experience for teachers who participated in the study. 
Table 4-8: Years of Experience Teaching

\begin{tabular}{|c|c|c|c|c|}
\hline Opioid Impact Zone & Size & $\begin{array}{c}\text { Years of } \\
\text { teaching } \\
(\text { Mean) }\end{array}$ & $\begin{array}{l}\text { Years of } \\
\text { teaching } \\
\text { (SD) }\end{array}$ & $\begin{array}{c}\text { Years of } \\
\text { teaching } \\
\text { (Range) }^{b}\end{array}$ \\
\hline Total & 450 & 9 & 7 & $1-25+$ \\
\hline Low & 150 & 8 & 6 & $1-25+$ \\
\hline Medium & 150 & 10 & 7 & $1-25+$ \\
\hline High & 150 & 10 & 7 & $1-25+$ \\
\hline
\end{tabular}

${ }^{a}$ The mean for years of teaching is low due to $25+$ years of teaching being recorded and analyzed as 25 years of teaching. This means that a teacher of 30 years would be counted as $25+$ years and analyzed at 25 years. ${ }^{\mathrm{b}}$ The range for years of teaching was capped at 25 years for online survey facilitation.

The design of the survey capped the years of service at 25 years, which means that teachers who taught 25,30 , and 35 years all were recorded as 25 years of teaching. The frequency table is shown below (see Table 4-9).

Table 4-9: Frequency of Teacher Years of Experience

\begin{tabular}{|c|c|c|c|c|}
\hline & Low & Medium & High & Total \\
\hline Total & $\mathbf{n}(\boldsymbol{\%})$ & $\mathbf{n}(\boldsymbol{\%})$ & $\mathbf{n}(\boldsymbol{\%})$ & $\mathbf{n}(\mathbf{\%})$ \\
\hline 1 & $7(5 \%)$ & $7(5 \%)$ & $6(4 \%)$ & $20(4 \%)$ \\
\hline 2 & $11(7 \%)$ & $8(5 \%)$ & $8(5 \%)$ & $27(6 \%)$ \\
\hline 3 & $14(9 \%)$ & $17(11 \%)$ & $23(15 \%)$ & $54(12 \%)$ \\
\hline 4 & $20(13 \%)$ & $12(8 \%)$ & $8(5 \%)$ & $40(9 \%)$ \\
\hline 5 & $14(9 \%)$ & $17(11 \%)$ & $20(13 \%)$ & $51(11 \%)$ \\
\hline 6 & $18(12 \%)$ & $10(7 \%)$ & $5(3 \%)$ & $33(7 \%)$ \\
\hline 7 & $7(5 \%)$ & $7(5 \%)$ & $7(5 \%)$ & $21(5 \%)$ \\
\hline 8 & $9(6 \%)$ & $5(3 \%)$ & $11(7 \%)$ & $25(6 \%)$ \\
\hline 9 & $2(1 \%)$ & $5(3 \%)$ & $4(3 \%)$ & $11(2 \%)$ \\
\hline 10 & $10(7 \%)$ & $6(4 \%)$ & $11(7 \%)$ & $27(6 \%)$ \\
\hline 11 & $2(1 \%)$ & $2(1 \%)$ & $1(1 \%)$ & $5(1 \%)$ \\
\hline 12 & $7(5 \%)$ & $11(7 \%)$ & $2(1 \%)$ & $20(4 \%)$ \\
\hline 13 & $2(1 \%)$ & $3(2 \%)$ & $6(4 \%)$ & $11(2 \%)$ \\
\hline 14 & $2(1 \%)$ & $5(3 \%)$ & $4(3 \%)$ & $11(2 \%)$ \\
\hline 15 & $2(1 \%)$ & $6(4 \%)$ & $1(1 \%)$ & $9(2 \%)$ \\
\hline 16 & $8(5 \%)$ & $2(1 \%)$ & $3(2 \%)$ & $13(3 \%)$ \\
\hline 17 & $2(1 \%)$ & $4(3 \%)$ & 0 & $6(1 \%)$ \\
\hline 18 & $1(1 \%)$ & $2(1 \%)$ & $7(5 \%)$ & $10(2 \%)$ \\
\hline
\end{tabular}




\begin{tabular}{|c|c|c|c|c|}
\hline 19 & 0 & $3(2 \%)$ & $2(1 \%)$ & $5(1 \%)$ \\
\hline 20 & $2(1 \%)$ & $3(2 \%)$ & $3(2 \%)$ & $8(2 \%)$ \\
\hline 21 & $1(1 \%)$ & 0 & $2(1 \%)$ & $3(1 \%)$ \\
\hline 22 & 0 & $2(1 \%)$ & $1(1 \%)$ & $3(1 \%)$ \\
\hline 23 & $2(1 \%)$ & 0 & $1(1 \%)$ & $3(1 \%)$ \\
\hline 24 & 0 & $2(1 \%)$ & $1(1 \%)$ & $3(1 \%)$ \\
\hline $25+$ & $7(5 \%)$ & $11(7 \%)$ & $13(9 \%)$ & $31(7 \%)$ \\
\hline
\end{tabular}

Sample teacher demographics compared to national teacher demographics. Nationally, in 2020 (Hussar et al., 2020), the profile of the teachers in the United States skewed toward females (76\%), White (79\%), with the majority of teachers in the age range of 30 to 49 years. For the sample of teachers in this study, the total sample $(\mathrm{N}=450)$ was similar, with the preponderance of respondents identifying as female (70.89\%), White (63.33\%), and the majority falling in the 31-50 years age-range (59.56\%). See Table 4-10 for a breakdown of this comparative information.

Table 4-10: Demographic Comparison National Teachers to Sample Teachers

\begin{tabular}{|l|c|c|}
\hline \multicolumn{1}{|c|}{ Characteristic } & $\begin{array}{c}\text { National } \\
\text { Total Percent }\end{array}$ & $\begin{array}{c}\text { Sample Total } \\
\text { Percent }\end{array}$ \\
\hline Gender & & \\
\hline Female & 76 & 70.89 \\
\hline Male & 24 & 27.56 \\
\hline Race/Ethnic Identity & 1 & \\
\hline American Indian or Alaskan Native (AI/AN) & 2 & 1.11 \\
\hline Asian & 7 & 4.67 \\
\hline Black or African American & 2 & 15.33 \\
\hline Combination of two or more races & 9 & 2.67 \\
\hline Hispanic/Latino/a & $\#$ & 0.22 \\
\hline Native Hawaiian or other Pacific Islander & 79 & 63.33 \\
\hline White & & \\
\hline
\end{tabular}

\# Rounds to zero.

The sample of teachers for this study was different than the larger population in the United States in that they are somewhat more diverse in gender and race/ethnicity. For age, there 
is a greater difference between the study sample and the national sample (see Tables 23 - 24). The study sample appears to be younger than teachers nationally, though, because of the age categories used by this study and the Hussar et al. data (2020).

Table 4-11: Teacher Study Sample Range

\begin{tabular}{|c|c|}
\hline $\begin{array}{c}\text { Sample Teacher } \\
\text { Age Range }\end{array}$ & $\begin{array}{c}\text { Sample Teacher } \\
\text { Percentage }\end{array}$ \\
\hline Less than 31 yrs. & 28.44 \\
\hline $31-50$ yrs. & 59.56 \\
\hline Over 51 yrs. & 12.0 \\
\hline
\end{tabular}

Table 4-12: Teacher National Age Range ${ }^{\mathrm{a}}$

\begin{tabular}{|c|c|}
\hline $\begin{array}{c}\text { National } \\
\text { Teacher Age } \\
\text { Range }\end{array}$ & $\begin{array}{c}\text { National } \\
\text { Teacher } \\
\text { Percentage }\end{array}$ \\
\hline Less than 30 yrs. & 15.3 \\
\hline $30-49$ yrs. & 54.0 \\
\hline Over 50. & 30.7 \\
\hline
\end{tabular}

${ }^{a}$ U.S. Department of Education, (2011).

\subsubsection{Teacher Report of Student Trauma (ACEs)}

The percent of teachers endorsing adverse childhood experiences present in students' households is reflected in Table 4-13. The ACEs scale helped establish that teachers were responders to student issues of need. Adverse childhood experiences include the following: physical or emotional abuse or neglect, loss of a parent, divorce or discord in the family, exposure to alcohol or drug abuse and mental illness in the home, or violence in the home or neighborhood (Bethell et al., 2017) with additional categories of incarcerated household member, felt racial/ethnic discrimination, and lived in foster care (Health Federation of Philadelphia and Philadelphia ACE Research and Data Committee, 2012). 
Table 4-13: Teachers' Perceptions of Student Adverse Childhood Experiences

\begin{tabular}{|c|c|c|c|c|}
\hline $\begin{array}{l}\text { Percent of teache } \\
\text { household }\end{array}$ & adorsing : & childhood ex & iences present in $\mathrm{s}$ & idents' \\
\hline ACEs Types & Total \% & Low Zone \% & Medium Zone \% & High Zone \% \\
\hline $\begin{array}{c}\text { Emotional } \\
\text { Abuse }\end{array}$ & $61 \%$ & $58 \%$ & $61 \%$ & $65 \%$ \\
\hline Physical Abuse & $44 \%$ & $41 \%$ & $46 \%$ & $44 \%$ \\
\hline Sexual Abuse & $29 \%$ & $27 \%$ & $31 \%$ & $30 \%$ \\
\hline $\begin{array}{c}\text { Emotional } \\
\text { Neglect }\end{array}$ & $50 \%$ & $55 \%$ & $48 \%$ & $49 \%$ \\
\hline $\begin{array}{c}\text { Physical } \\
\text { Neglect }\end{array}$ & $34 \%$ & $31 \%$ & $33 \%$ & $37 \%$ \\
\hline $\begin{array}{l}\text { Domestic } \\
\text { Violence }\end{array}$ & $42 \%$ & $42 \%$ & $45 \%$ & $40 \%$ \\
\hline $\begin{array}{c}\text { Household } \\
\text { Substance } \\
\text { Abuse }\end{array}$ & $47 \%$ & $48 \%$ & $46 \%$ & $46 \%$ \\
\hline $\begin{array}{c}\text { Household } \\
\text { Mental Illness }\end{array}$ & $38 \%$ & $35 \%$ & $39 \%$ & $40 \%$ \\
\hline $\begin{array}{c}\text { Incarcerated } \\
\text { Household } \\
\text { member }\end{array}$ & $41 \%$ & $41 \%$ & $41 \%$ & $41 \%$ \\
\hline $\begin{array}{c}\text { Witness } \\
\text { Violence }\end{array}$ & $32 \%$ & $29 \%$ & $36 \%$ & $33 \%$ \\
\hline $\begin{array}{l}\text { Racial Ethnic } \\
\text { Discrimination }\end{array}$ & $39 \%$ & $43 \%$ & $42 \%$ & $32 \%$ \\
\hline $\begin{array}{c}\text { Adverse } \\
\text { Neighborhood } \\
\text { Experience }\end{array}$ & $28 \%$ & $25 \%$ & $26 \%$ & $35 \%$ \\
\hline $\begin{array}{c}\text { Lived in Foster } \\
\text { Care }\end{array}$ & $48 \%$ & $49 \%$ & $53 \%$ & $42 \%$ \\
\hline $\begin{array}{c}\text { Experienced } \\
\text { Death in Family }\end{array}$ & $52 \%$ & $51 \%$ & $57 \%$ & $47 \%$ \\
\hline None & $8 \%$ & $9 \%$ & $9 \%$ & $7 \%$ \\
\hline
\end{tabular}




\subsection{Secondary Trauma and Teachers}

To evaluate the level of STS in teachers and to examine the relationship between STS and characteristics of teachers, the Secondary Traumatic Stress Scale (STSS) was used (Bride et al., 2004; Bride, 2007). The Secondary Traumatic Stress Scale (STSS; Bride et al., 2004) is a 17item self-report instrument that can assess symptoms associated with secondary traumatic stress (STS). These symptoms, which can be the result of working with people who have experienced traumatic events, are assessed along three subscales that correspond to the PTSD diagnostic criteria in the Diagnostic and Statistical Manual of Mental Disorders, 4th edition, revised (DSMIV-TR; American Psychiatric Association, 2000). The three subscales are intrusion, avoidance, and arousal; they are indicated by the respondents reporting the frequency, on a five-point Likert scale, endorsing their self-report (Bride, 2007). For this study, 450 participants returned completed STSS instruments, with 150 participants in each of three opioid epidemic zones (low, medium, and high). For the full STSS, with 450 respondents, there was a sample mean score of 42.06 with a standard deviation of 14.79 (95\% CI 40.7 to 43.4). This score will be explored more fully by individual question, by opioid zone, and by subscale.

\subsubsection{Individual Question Results}

Table 4-14 shows the response to each question of the instrument, both for the total study $(\mathrm{N}=450)$, followed by tables with response mean and standard deviation by subscale for each of the three opioid zones (see Tables 4-14 through 4-17). The tables show the 17-item STSS questions as they relate to the PTSD diagnostic criteria of intrusion, avoidance, and arousal. Each of the STSS question numbers appears in parentheses after the criterion. 
Table 4-14: Secondary Traumatic Stress Scale by Question for Total Sample (N = 450)

\begin{tabular}{|c|c|c|c|c|c|}
\hline & Never & Rarely & Occasionally & Often & $\begin{array}{l}\text { Very } \\
\text { Often }\end{array}$ \\
\hline STSS Subscale & $\%(\mathrm{~N})$ & $\%(\mathrm{~N})$ & $\%(\mathrm{~N})$ & $\%(\mathrm{~N})$ & $\%(\mathrm{~N})$ \\
\hline \multicolumn{6}{|l|}{ Intrusion Subscale } \\
\hline Intrusive thoughts about students (10) & $\begin{array}{l}15.6 \\
(70)\end{array}$ & $\begin{array}{l}12.7 \\
(57)\end{array}$ & $34.4(155)$ & $\begin{array}{c}25.6 \\
(115)\end{array}$ & $\begin{array}{l}11.8 \\
(53)\end{array}$ \\
\hline Disturbing dreams about students (13) & $\begin{array}{c}36.4 \\
(164)\end{array}$ & $\begin{array}{l}26.9 \\
(121)\end{array}$ & $22.9(103)$ & $\begin{array}{l}8.9 \\
(40)\end{array}$ & $\begin{array}{l}4.9 \\
(22)\end{array}$ \\
\hline Sense of reliving students' trauma (3) & $\begin{array}{c}22.4 \\
(101)\end{array}$ & $\begin{array}{l}23.3 \\
(105)\end{array}$ & $28.7(129)$ & $\begin{array}{l}18.4 \\
(83)\end{array}$ & $\begin{array}{l}7.1 \\
(32)\end{array}$ \\
\hline Cued psychological distress (6) & $\begin{array}{l}28.7 \\
(129)\end{array}$ & $\begin{array}{c}28.4 \\
(128)\end{array}$ & $24.4(110)$ & $\begin{array}{l}12.7 \\
(57)\end{array}$ & $\begin{array}{l}5.8 \\
(26)\end{array}$ \\
\hline Cued physiological reactivity (2) & $\begin{array}{l}20.9 \\
(94)\end{array}$ & $\begin{array}{l}24.4 \\
(110)\end{array}$ & $29.1(131)$ & $\begin{array}{l}19.3 \\
(87)\end{array}$ & $\begin{array}{l}6.2 \\
(28)\end{array}$ \\
\hline \multicolumn{6}{|l|}{ Avoidance Subscale } \\
\hline Avoidance of students (14) & $\begin{array}{l}38.2 \\
(172)\end{array}$ & $\begin{array}{l}25.1 \\
(113)\end{array}$ & $22.4(101)$ & $\begin{array}{l}9.3 \\
(42)\end{array}$ & $\begin{array}{l}4.9 \\
(22)\end{array}$ \\
\hline $\begin{array}{l}\text { Avoidance of people, places, things } \\
\text { (12) }\end{array}$ & $\begin{array}{l}37.6 \\
(169)\end{array}$ & $\begin{array}{l}29.1 \\
(131)\end{array}$ & $19.1(86)$ & $\begin{array}{l}10.0 \\
(45)\end{array}$ & $\begin{array}{l}4.2 \\
(19)\end{array}$ \\
\hline $\begin{array}{l}\text { Inability to recall student information } \\
\text { (17) }\end{array}$ & $\begin{array}{l}40.9 \\
(184)\end{array}$ & $\begin{array}{l}25.1 \\
(113)\end{array}$ & $20.0(90)$ & $\begin{array}{l}9.3 \\
(42)\end{array}$ & $\begin{array}{l}4.7 \\
(21)\end{array}$ \\
\hline Diminished activity level (9) & $\begin{array}{r}27.6 \\
(124) \\
\end{array}$ & $\begin{array}{c}25.1 \\
(113)\end{array}$ & $28.4(128)$ & $\begin{array}{l}13.8 \\
(62) \\
\end{array}$ & $\begin{array}{l}5.1 \\
(23) \\
\end{array}$ \\
\hline Detachment from others (7) & $\begin{array}{c}35.3 \\
(159)\end{array}$ & $\begin{array}{l}28.9 \\
(130)\end{array}$ & $19.8(89)$ & $\begin{array}{l}11.3 \\
(51)\end{array}$ & $\begin{array}{l}4.7 \\
(21)\end{array}$ \\
\hline Emotional numbing (1) & $\begin{array}{l}18.7 \\
(84)\end{array}$ & $\begin{array}{l}28.0 \\
(126)\end{array}$ & $35.1(158)$ & $\begin{array}{l}13.3 \\
(60)\end{array}$ & $\begin{array}{l}4.9 \\
(22)\end{array}$ \\
\hline Foreshortened future (5) & $\begin{array}{l}17.1 \\
(77)\end{array}$ & $\begin{array}{l}24.9 \\
(112)\end{array}$ & $33.1(149)$ & $\begin{array}{l}17.3 \\
(78)\end{array}$ & $\begin{array}{l}7.6 \\
(34)\end{array}$ \\
\hline \multicolumn{6}{|l|}{ Arousal Subscale } \\
\hline Difficulty sleeping (4) & $\begin{array}{l}14.0 \\
(63) \\
\end{array}$ & $\begin{array}{l}19.3 \\
(87) \\
\end{array}$ & $36.2(163)$ & $\begin{array}{l}20.0 \\
(90) \\
\end{array}$ & $\begin{array}{l}10.4 \\
(47)\end{array}$ \\
\hline Irritability (15) & $\begin{array}{c}25.6 \\
(115)\end{array}$ & $\begin{array}{c}29.3 \\
(132)\end{array}$ & $27.8(125)$ & $\begin{array}{l}10.2 \\
(46)\end{array}$ & $\begin{array}{r}7.1 \\
(32)\end{array}$ \\
\hline Difficulty concentrating (11) & $\begin{array}{l}20.4 \\
(92)\end{array}$ & $\begin{array}{c}26.7 \\
(120)\end{array}$ & $29.1(131)$ & $\begin{array}{l}15.8 \\
(71)\end{array}$ & $\begin{array}{c}8.0 \\
(36)\end{array}$ \\
\hline
\end{tabular}




\begin{tabular}{|l|c|c|c|c|c|}
\hline Hypervigilance (16) & $\begin{array}{c}29.6 \\
(133)\end{array}$ & $\begin{array}{c}25.8 \\
(116)\end{array}$ & $25.8(116)$ & 12.9 & 6.0 \\
& 31.8 & 27.3 & $24.9(112)$ & 9.6 & 6.4 \\
Easily startled (8) & $(143)$ & $(123)$ & & $(43)$ & $(29)$ \\
\hline
\end{tabular}

Note. Range for the instrument: 17-85; Mean for the Total $(\mathrm{N}=450)$ : 42.06; SD for the Total $(\mathrm{N}=450): 14.79$.

${ }^{a}$ Total refers to the total sample, $\mathrm{N}=450$.

The questions with the highest overall percentage endorsed the following symptom criteria at occasionally or more often (score of 3, 4, or 5): intrusive thoughts $(71.8 \%$ ) and difficulty sleeping (66.6\%). Over $50 \%$ of teachers endorsed these symptoms occasionally or more often: reliving student trauma (54.2\%), physiological symptoms when thinking about work $(54.6 \%)$, emotional numbing $(53.3 \%)$, discouragement about the future $(58.0 \%)$, and difficulty concentrating $(52.9 \%)$.

In addition to the report of individual question results for all respondents $(\mathrm{N}=450)$, each opioid zone was analyzed individually for the subscale answers on the STSS (see Tables 27 29).

Table 4-15: Secondary Traumatic Stress Scale by Question for Low Opioid Epidemic Zone $(\mathrm{n}=$

\begin{tabular}{|l|c|c|c|c|c|}
\hline & Never & Rarely & $\begin{array}{c}\text { Occasional } \\
\text { ly }\end{array}$ & Often & $\begin{array}{c}\text { Very } \\
\text { Often }\end{array}$ \\
\hline STSS Subscale & \% (n) & \% (n) & $\%(\mathbf{n})$ & \% (n) & $\%(\mathbf{m})$ \\
\hline Intrusion Subscale & & & & & \\
\hline Intrusive thoughts about students (10) & 16.0 & 10.0 & $38.7(58)$ & 25.3 & 10.0 \\
& $(24)$ & $(15)$ & & $(38)$ & $(15)$ \\
\hline Disturbing dreams about students & 39.3 & 22.7 & $24.0(36)$ & $9.3(14)$ & $4.7(7)$ \\
$(13)$ & $(59)$ & $(34)$ & & & \\
\hline Sense of reliving students' trauma (3) & 20.7 & 23.3 & $31.3(47)$ & 16.0 & $8.7(13)$ \\
& $(31)$ & $(35)$ & & $(24)$ & \\
\hline Cued psychological distress (6) & 30.0 & 28.0 & $24.7(37)$ & 14.0 & $3.3(5)$ \\
& $(45)$ & $(42)$ & & $(21)$ & \\
\hline
\end{tabular}




\begin{tabular}{|c|c|c|c|c|c|}
\hline Cued physiological reactivity (2) & $\begin{array}{l}20.0 \\
(30)\end{array}$ & $\begin{array}{l}26.0 \\
(39)\end{array}$ & $26.7(40)$ & $\begin{array}{l}22.7 \\
(34)\end{array}$ & $4.7(7)$ \\
\hline \multicolumn{6}{|l|}{ Avoidance Subscale } \\
\hline Avoidance of students (14) & $\begin{array}{l}40.0 \\
(60)\end{array}$ & $\begin{array}{l}18.0 \\
(27)\end{array}$ & $28.7(43)$ & $9.3(14)$ & $4.0(6)$ \\
\hline $\begin{array}{l}\text { Avoidance of people, places, things } \\
\text { (12) }\end{array}$ & $\begin{array}{l}39.3 \\
(59)\end{array}$ & $\begin{array}{l}26.0 \\
(39)\end{array}$ & $20.7(31)$ & $\begin{array}{l}10.0 \\
(15)\end{array}$ & $4.0(6)$ \\
\hline $\begin{array}{l}\text { Inability to recall student information } \\
\text { (17) }\end{array}$ & $\begin{array}{l}41.3 \\
(62)\end{array}$ & $\begin{array}{l}23.3 \\
(35)\end{array}$ & $19.3(29)$ & $\begin{array}{l}10.7 \\
(16)\end{array}$ & $5.3(8)$ \\
\hline Diminished activity level (9) & $\begin{array}{l}27.3 \\
(41)\end{array}$ & $\begin{array}{l}22.7 \\
(34)\end{array}$ & $32.0(48)$ & $\begin{array}{l}12.7 \\
(19)\end{array}$ & $5.3(8)$ \\
\hline Detachment from others (7) & $\begin{array}{l}33.3 \\
(50)\end{array}$ & $\begin{array}{l}23.3 \\
(35)\end{array}$ & $24.0(36)$ & $\begin{array}{l}14.0 \\
(21)\end{array}$ & $5.3(8)$ \\
\hline Emotional numbing (1) & $\begin{array}{l}18.7 \\
(28)\end{array}$ & $\begin{array}{l}28.0 \\
(42)\end{array}$ & $32.7(49)$ & $\begin{array}{l}14.7 \\
(22)\end{array}$ & $6.0(9)$ \\
\hline Foreshortened future (5) & $\begin{array}{l}18.7 \\
(28)\end{array}$ & $\begin{array}{l}22.7 \\
(34)\end{array}$ & $36.0(54)$ & $\begin{array}{l}16.0 \\
(24)\end{array}$ & $6.7(10)$ \\
\hline \multicolumn{6}{|l|}{ Arousal Subscale } \\
\hline Difficulty sleeping (4) & $\begin{array}{l}14.7 \\
(22)\end{array}$ & $\begin{array}{l}22.7 \\
(34)\end{array}$ & $33.3(50)$ & $\begin{array}{l}18.7 \\
(28)\end{array}$ & $\begin{array}{l}10.7 \\
(16)\end{array}$ \\
\hline Irritability (15) & $\begin{array}{l}27.3 \\
(41)\end{array}$ & $\begin{array}{l}25.3 \\
(38)\end{array}$ & $28.0(42)$ & $\begin{array}{l}12.7 \\
(19)\end{array}$ & $6.7(10)$ \\
\hline Difficulty concentrating (11) & $\begin{array}{l}24.7 \\
(37)\end{array}$ & $\begin{array}{l}26.7 \\
(40)\end{array}$ & $23.3(35)$ & $\begin{array}{l}16.7 \\
(25)\end{array}$ & $8.7(13)$ \\
\hline Hypervigilance (16) & $\begin{array}{l}29.3 \\
(44)\end{array}$ & $\begin{array}{l}28.0 \\
(42)\end{array}$ & $24.7(37)$ & $\begin{array}{l}12.7 \\
(19)\end{array}$ & $5.3(8)$ \\
\hline Easily startled (8) & $\begin{array}{l}28.7 \\
(43)\end{array}$ & $\begin{array}{l}28.7 \\
(43)\end{array}$ & $24.0(36)$ & $\begin{array}{l}11.3 \\
(17)\end{array}$ & $7.3(11)$ \\
\hline
\end{tabular}

Note. Range for the instrument: 17-85; Mean for the Total $(\mathrm{N}=450)$ : 42.06; SD for the Total $(\mathrm{N}=450)$ : 14.79 . 
Table 4-16: Secondary Traumatic Stress Scale by Question for Medium Opioid Epidemic Zone

\begin{tabular}{|l|c|c|c|c|c|}
\hline & Never & Rarely & Occasionally & Often & $\begin{array}{c}\text { Very } \\
\text { Often }\end{array}$ \\
\hline STSS Subscale & $\mathbf{\%}(\mathbf{n})$ & $\mathbf{\%}(\mathbf{n})$ & $\mathbf{\%}(\mathbf{n})$ & $\mathbf{\%}(\mathbf{n})$ & $\mathbf{\%}(\mathbf{n})$ \\
\hline Intrusion Subscale & & & & & \\
\hline Intrusive thoughts about students (10) & 12.7 & 14.0 & $34.7(52)$ & 26.0 & 12.7 \\
& $(19)$ & $(21)$ & & $(39)$ & $(19)$ \\
\hline Disturbing dreams about students (13) & 38.7 & 32.7 & $18.7(28)$ & $4.7(7)$ & $5.3(8)$ \\
& $(58)$ & $(49)$ & & & \\
\hline Sense of reliving students' trauma (3) & 24.7 & 24.7 & $30.0(45)$ & 14.7 & $6.0(9)$ \\
& $(37)$ & $(37)$ & & $(22)$ & \\
\hline Cued psychological distress (6) & 30.7 & 34.7 & $21.3(32)$ & 8.7 & $4.7(7)$ \\
& $(46)$ & $(52)$ & & $(13)$ & \\
\hline Cued physiological reactivity (2) & 20.7 & 25.3 & $34.7(52)$ & 16.0 & $3.3(5)$ \\
& $(31)$ & $(38)$ & & $(24)$ & \\
\hline Avoidance Subscale & & & & & \\
\hline Avoidance of students (14) & 40.0 & 29.3 & $20.7(31)$ & $6.0(9)$ & $4.0(6)$ \\
& $(60)$ & $(44)$ & & & \\
\hline Avoidance of people, places, things & 42.7 & 34.0 & $14.7(22)$ & 6.7 & $2.0(3)$ \\
& $(64)$ & $(51)$ & & $(10)$ & \\
\hline In) & 44.7 & 29.3 & $16.7(25)$ & $6.0(9)$ & $3.3(5)$ \\
\hline (17) & $(67)$ & $(44)$ & & & \\
\hline Diminished activity level (9) & 27.3 & 30.0 & $27.3(41)$ & 12.0 & $3.3(5)$ \\
& $(41)$ & $(45)$ & & $(18)$ & \\
\hline Detachment from others (7) & 38.0 & 34.0 & $17.3(26)$ & 6.7 & $4.0(6)$ \\
& $(57)$ & $(51)$ & & $(10)$ & \\
\hline Emotional numbing (1) & 19.3 & 28.7 & $40.0(60)$ & 8.7 & $3.3(5)$ \\
& $(29)$ & $(43)$ & & $(13)$ & \\
\hline Foreshortened future (5) & 18.0 & 28.0 & $32.7(49)$ & 15.3 & $6.0(9)$ \\
& $(27)$ & $(42)$ & & $(23)$ & \\
\hline Arousal Subscale & & & & & \\
\hline Difficulty sleeping (4) & 13.3 & 20.7 & $36.0(54)$ & 22.0 & $8.0(12)$ \\
& $(20)$ & $(31)$ & & $(33)$ & \\
\hline Irritability (15) & 24.7 & 32.7 & $30.7(46)$ & $6.0(9)$ & $6.0(9)$ \\
& $(37)$ & $(49)$ & & & \\
\hline
\end{tabular}




\begin{tabular}{|l|c|c|c|c|c|}
\hline Difficulty concentrating (11) & $\begin{array}{l}16.7 \\
(25)\end{array}$ & $\begin{array}{l}30.7 \\
(46)\end{array}$ & $36.7(55)$ & $\begin{array}{l}10.7 \\
(16)\end{array}$ & $5.3(8)$ \\
\hline Hypervigilance (16) & 31.3 & 28.7 & $24.7(37)$ & 10.0 & $5.3(8)$ \\
& $(47)$ & $(43)$ & & $(15)$ & \\
\hline Easily startled (8) & 34.0 & 28.7 & $25.3(38)$ & $6.0(9)$ & $6.0(9)$ \\
& $(51)$ & $(43)$ & & & \\
\hline
\end{tabular}

Note. Range for the instrument: 17-85; Mean for the Total $(\mathrm{N}=450)$ : 42.06; SD for the Total $(\mathrm{N}=450)$ : 14.79 .

Table 4-17: Secondary Traumatic Stress Scale by Question for High Opioid Epidemic Zone $(\mathrm{n}=$ 150)

\begin{tabular}{|c|c|c|c|c|c|}
\hline & Never & Rarely & Occasionally & Often & $\begin{array}{l}\text { Very } \\
\text { Often }\end{array}$ \\
\hline STSS Subscale & $\%(n)$ & $\%(n)$ & $\%(n)$ & $\%(n)$ & $\%(n)$ \\
\hline \multicolumn{6}{|l|}{ Intrusion Subscale } \\
\hline $\begin{array}{l}\text { Intrusive thoughts about students } \\
\text { (10) }\end{array}$ & $\begin{array}{l}18.0 \\
(27)\end{array}$ & $\begin{array}{l}14.0 \\
(21)\end{array}$ & $30.0(45)$ & $\begin{array}{l}25.3 \\
(38)\end{array}$ & $\begin{array}{l}12.7 \\
(19)\end{array}$ \\
\hline $\begin{array}{l}\text { Disturbing dreams about students } \\
\text { (13) }\end{array}$ & $\begin{array}{l}31.3 \\
(47)\end{array}$ & $\begin{array}{l}25.3 \\
(38)\end{array}$ & $26.0(39)$ & $\begin{array}{l}12.7 \\
(19)\end{array}$ & $4.7(7)$ \\
\hline $\begin{array}{l}\text { Sense of reliving students' trauma } \\
\text { (3) }\end{array}$ & $\begin{array}{l}22.0 \\
(33)\end{array}$ & $\begin{array}{l}22.0 \\
(33)\end{array}$ & $24.7(37)$ & $\begin{array}{l}24.7 \\
(37)\end{array}$ & $\begin{array}{l}6.7 \\
(10)\end{array}$ \\
\hline Cued psychological distress (6) & $\begin{array}{l}25.3 \\
(38)\end{array}$ & $\begin{array}{l}22.7 \\
(34)\end{array}$ & $27.3(41)$ & $\begin{array}{l}15.3 \\
(23)\end{array}$ & $\begin{array}{l}9.3 \\
(14)\end{array}$ \\
\hline Cued physiological reactivity (2) & $\begin{array}{l}22.0 \\
(33)\end{array}$ & $\begin{array}{l}22.0 \\
(33)\end{array}$ & $26.0(39)$ & $\begin{array}{l}19.3 \\
(29)\end{array}$ & $\begin{array}{l}10.7 \\
(16)\end{array}$ \\
\hline \multicolumn{6}{|l|}{ Avoidance Subscale } \\
\hline Avoidance of students (14) & $\begin{array}{l}34.7 \\
(52)\end{array}$ & $\begin{array}{l}28.0 \\
(42)\end{array}$ & $18.0(27)$ & $\begin{array}{l}12.7 \\
(19)\end{array}$ & $\begin{array}{l}6.7 \\
(10)\end{array}$ \\
\hline $\begin{array}{l}\text { Avoidance of people, places, things } \\
\text { (12) }\end{array}$ & $\begin{array}{l}30.7 \\
(46)\end{array}$ & $\begin{array}{l}27.3 \\
(41)\end{array}$ & $22.0(33)$ & $\begin{array}{l}13.3 \\
(20)\end{array}$ & $\begin{array}{l}6.7 \\
(10)\end{array}$ \\
\hline $\begin{array}{l}\text { Inability to recall student } \\
\text { information }(17)\end{array}$ & $\begin{array}{l}36.7 \\
(55)\end{array}$ & $\begin{array}{l}22.7 \\
(34)\end{array}$ & $24.0(36)$ & $\begin{array}{l}11.3 \\
(17)\end{array}$ & $5.3(8)$ \\
\hline Diminished activity level (9) & $\begin{array}{l}28.0 \\
(42)\end{array}$ & $\begin{array}{l}22.7 \\
(34)\end{array}$ & $26.0(39)$ & $\begin{array}{l}16.7 \\
(25)\end{array}$ & $\begin{array}{l}6.7 \\
(10)\end{array}$ \\
\hline Detachment from others (7) & $\begin{array}{l}34.7 \\
(52)\end{array}$ & $\begin{array}{l}29.3 \\
(44)\end{array}$ & $18.0(27)$ & $\begin{array}{l}13.3 \\
(20)\end{array}$ & $4.7(7)$ \\
\hline Emotional numbing (1) & $\begin{array}{l}18.0 \\
(27)\end{array}$ & $\begin{array}{l}27.3 \\
(43)\end{array}$ & $32.7(49)$ & $\begin{array}{l}16.7 \\
(25)\end{array}$ & $5.3(8)$ \\
\hline
\end{tabular}




\begin{tabular}{|l|l|l|c|c|c|}
\hline Foreshortened future (5) & $\begin{array}{l}14.7 \\
(22)\end{array}$ & $\begin{array}{l}24.0 \\
(36)\end{array}$ & $30.7(46)$ & $\begin{array}{l}20.7 \\
(31)\end{array}$ & $\begin{array}{c}10.0 \\
(15)\end{array}$ \\
\hline Arousal Subscale & & & & & \\
\hline Difficulty sleeping (4) & 14.0 & 14.7 & $39.3(59)$ & 19.3 & 12.7 \\
& $(21)$ & $(22)$ & & $(29)$ & $(19)$ \\
\hline Irritability (15) & 24.7 & 30.0 & $24.7(37)$ & 12.0 & 8.7 \\
& $(37)$ & $(45)$ & & $(18)$ & $(13)$ \\
\hline Difficulty concentrating (11) & 20.0 & 22.7 & $27.3(41)$ & 20.0 & 10.0 \\
& $(30)$ & $(34)$ & & $(30)$ & $(15)$ \\
\hline Hypervigilance (16) & 28.0 & 20.7 & $28.0(42)$ & 16.0 & 7.3 \\
& $(42)$ & $(31)$ & & $(24)$ & $(11)$ \\
\hline Easily startled (8) & 32.7 & 24.7 & $25.3(38)$ & 11.3 & $6.0(9)$ \\
& $(49)$ & $(37)$ & & $(17)$ & \\
\hline
\end{tabular}

Note. Range for the instrument: 17-85; Mean for the Total ( $\mathrm{N}=450)$ : 42.06; SD for the Total ( $\mathrm{N}=450)$ : 14.79 .

Full scale STSS results and interpretation. Table 4-18 reports the result for the sample on the full-scale STSS for the three opioid zones and the total sample. The mean total score for the sample was 42.06, with a standard deviation of 14.79. This result indicates a moderate level of stress, according to Bride et al. (2004). The range for the instrument is 17-85.

Table 4-18: Results of the STSS Scale by Opioid Zone

\begin{tabular}{|l|c|c|c|c|}
\hline \multicolumn{1}{|c|}{ Opioid Zone } & $\boldsymbol{N}$ & $\boldsymbol{M}$ & $\boldsymbol{S D}$ & Interpretation \\
\hline Low & 150 & 42.16 & 14.71 & Strong- Moderate \\
\hline Medium & 150 & 40.23 & 13.44 & Moderate \\
\hline High & 150 & 43.78 & 16.00 & Moderate/High \\
\hline Total (All three zones) & 450 & 42.06 & 14.79 & Strong-Moderate \\
\hline
\end{tabular}

Inspecting the items scores in Table 4-18, the means in all three zones are at a moderate level or above. This moderate level can be understood through the lens of point prevalence using the summing/percentile method for scoring (Bride, 2007; Centers for Disease Control and Prevention, 2012b). The scores and their meanings represent a number of cases at one point in time, with that time being the time of the survey. 
Subscale STSS results and interpretation. The STSS scores can be interpreted in three ways: percentile interpretation, cutoff interpretation, and algorithm interpretation. Each of the methods is represented below.

Percentile interpretation of the STSS subscales. Table 4-19 reports the result for the sample on each subscale of the STSS for the three opioid zones and the total sample. The avoidance subscale is in the low-moderate range, while the arousal subscale for all 450 respondents is in the moderate range. The intrusion subscale is right at the severe range, although 12.93 is .07 less than the 13.00 cutoff for the $95^{\text {th }}$ percentile. If the standard deviation $(S D=$ 4.51) is factored in, the interpretation of high/severe is sound for some respondents, with a less severed interpretation for others (see Table 4-20).

Table 4-19: Results of the STSS Scale and Total Scores

\begin{tabular}{|l|c|c|c|c|}
\hline \multicolumn{1}{|c}{ STSS } & \multicolumn{1}{c}{$\boldsymbol{N}$} & \multicolumn{1}{c|}{$\boldsymbol{M} \boldsymbol{D}$} & Interpretation \\
\hline Intrusion Scale & 450 & 12.93 & 4.51 & High/Severe \\
\hline Avoidance Scale & 450 & 16.40 & 6.32 & Weak Moderate \\
\hline Arousal Scale & 450 & 12.73 & 4.77 & Moderate \\
\hline STSS Total Score & 450 & 42.06 & 14.79 & Moderate/High \\
\hline
\end{tabular}

The mean and standard deviation was calculated from the STSS scores for the 450 respondents. The results are listed below in Table 4-20. Bride (2007) offered a guide for the interpretation of scores using this summed/percentile method by comparing respondents' scores to normative scores (Table 4-20). Then the scores are classified into the percentile ranges in the table; if the scores fall at the $50^{\text {th }}$ percentile or below, they are interpreted as little to no STSS; if $51^{\text {st }}-75^{\text {th }}$ percentile, mild STSS; and if $76^{\text {th }}-90^{\text {th }}$ percentile, moderate. A high level of STSS is interpreted by a score that falls in the $91^{\text {st }}-95^{\text {th }}$ percentile, and any score over the $95^{\text {th }}$ percentile is severe. 
Table 4-20: Bride's (2007) Interpretation for the STSS Subscales

\begin{tabular}{|c|c|c|c|c|c|c|c|c|c|c|}
\hline \multicolumn{11}{|c|}{$\begin{array}{l}\text { Means, Standard Deviations, Ranges, and Percentiles } \\
\text { for the Intrusion, Avoidance, and Arousal Subscales and the Full STSS }\end{array}$} \\
\hline \multicolumn{7}{|c|}{ Range } & \multicolumn{4}{|c|}{ Percentile } \\
\hline $\begin{array}{c}\text { STSS } \\
\text { Subscale }\end{array}$ & $\begin{array}{c}\text { Question } \\
\#\end{array}$ & $M$ & $(S D)$ & Possible & Observed & $\begin{array}{c}25^{\text {th }} \\
\text { Littlle } \\
\text { to no } \\
\text { STSS }\end{array}$ & $\begin{array}{c}50^{\text {th }} \\
\text { Mild } \\
\text { STSS }\end{array}$ & $\begin{array}{c}75^{\text {th }} \\
\text { Moderate } \\
\text { STSS }\end{array}$ & $\begin{array}{c}90^{\text {th }} \\
\text { High } \\
\text { STSS }\end{array}$ & $\begin{array}{c}95^{\text {th }} \\
\text { Severe } \\
\text { STSS }\end{array}$ \\
\hline $\begin{array}{l}\text { Intrusion } \\
\text { Subscale }\end{array}$ & $\begin{array}{l}2,3,6 \\
10,13\end{array}$ & 8.18 & (3.04) & $5-25$ & $5-21$ & 6.00 & 7.00 & 11.00 & 12.00 & 13.00 \\
\hline $\begin{array}{l}\text { Avoidance } \\
\text { Subscale }\end{array}$ & $\begin{array}{c}1,5,7 \\
9,12,14\end{array}$ & 12.58 & $(5.00)$ & $7-35$ & $7-31$ & 8.00 & 12.00 & 16.00 & 20.00 & 22.00 \\
\hline $\begin{array}{l}\text { Arousal } \\
\text { Subscale }\end{array}$ & $\begin{array}{c}4,8,11 \\
15,16\end{array}$ & 9.93 & $(3.56)$ & $5-25$ & $5-24$ & 6.00 & 8.00 & 11.00 & 14.00 & 16.00 \\
\hline $\begin{array}{l}\text { Total } \\
\text { STSS }\end{array}$ & $\begin{array}{l}1-17 \\
\text { (all) }\end{array}$ & 29.69 & (10.74) & $17-85$ & $17-24$ & 21.00 & 27.00 & 37.00 & 43.80 & 48.40 \\
\hline
\end{tabular}

a Table is adapted from "Prevalence of Secondary Traumatic Stress among Social Workers" by Bride (2007), Social Work, 52(1), p. 68. Original chart can be viewed at https://www.researchgate.net/profile/Brian_Bride/publication/6419610_Prevalence_of_Secondary_Traumatic_Stres s_among_Social_Workers/links/5736177e08ae298602e09fcc.pdf

Cutoff scoring measure of STS in teachers. Unlike the summing/percentile method, which requires averages (means) for determining the level of endorsement of STS in teachers and is then compared to percentiles, the cutoff method relies on the score of 38 as a marker for diagnostic levels. If a total score on the STSS is 38 or higher, then interventions could be therapeutically indicated for STSS (Bride, 2007; Bride et al., 2007). Table 4-21 shows the scores for the sample, using the cutoff method.

Table 4-21: STSS of Teachers Indicated by Using the Cutoff Method

\begin{tabular}{|l|c|c|c|c|}
\hline Teachers & $\begin{array}{c}\text { Scored } \mathbf{> 3 8} \\
\text { Number }\end{array}$ & $\begin{array}{c}\text { Scored } \mathbf{3 8} \\
\text { Percentage }\end{array}$ & $\begin{array}{c}\text { Scored }<\mathbf{3 7} \\
\text { Number }\end{array}$ & $\begin{array}{c}\text { Scored <37 } \\
\text { Percentage }\end{array}$ \\
\hline Low & 90 & $60.00 \%$ & 60 & $40.00 \%$ \\
\hline Medium & 84 & $56.00 \%$ & 66 & $44.00 \%$ \\
\hline High & 94 & $62.67 \%$ & 56 & $37.33 \%$ \\
\hline Total & 268 & $59.56 \%$ & 182 & $40.44 \%$ \\
\hline
\end{tabular}


Bride (2007) noted the following regarding the cutoff method:

That is, using a cutoff value of 38,93 percent of those who met the core criteria for PTSD using the algorithm approach would be correctly identified as having PTSD and 91 percent of those who did not meet the core criteria for PTSD would be identified as not having PTSD. (p. 68)

By this indicator, almost $60 \%$ of the respondents meet the criteria for STSS (and PTSD). In the high opioid zone, over $60 \%$ of the teachers endorse this interpretation.

Algorithm scoring measure of STS in teachers. The third scoring method is an algorithm that utilizes the three subscales (see Table 4-22). Bride (2007) described algorithm scoring, which is based on PTSD diagnostic levels (Bride et al., 2007). If teachers answer a particular question with a score of 3,4 , or 5 , then the item is considered endorsed. For the intrusion scale (questions 2, 3, 6, 10, 13), teachers only need to endorse at least one item; for the avoidance scale (questions $1,5,7,9,12,14$ ), teachers must endorse three or more items; and for the arousal scale (questions $4,8,11,15,16$ ), two or more items must be answered at 3, 4, or 5 .

Table 4-22: Algorithm Scoring of STSS ${ }^{\mathrm{a}}$

\begin{tabular}{|c|c|c|c|c|c|c|c|c|}
\hline & \multicolumn{2}{|c|}{ Low } & \multicolumn{2}{c|}{ Medium } & \multicolumn{2}{c|}{ High } & \multicolumn{2}{c|}{ Total } \\
\hline STSS Subscale & $\mathbf{N}$ & $\mathbf{P}$ & $\mathbf{N}$ & $\mathbf{P}$ & $\mathbf{N}$ & $\mathbf{P}$ & $\mathbf{N}$ & P \\
\hline Intrusion & 132 & $88.00 \%$ & 124 & $82.67 \%$ & 132 & $88.00 \%$ & 388 & $86.22 \%$ \\
\hline Avoidance & 85 & $56.67 \%$ & 64 & $42.67 \%$ & 80 & $53.33 \%$ & 229 & $50.89 \%$ \\
\hline Arousal & 95 & $63.33 \%$ & 92 & $61.33 \%$ & 104 & $69.33 \%$ & 291 & $64.67 \%$ \\
\hline $\begin{array}{c}\text { Diagnostic } \\
\text { Level }\end{array}$ & 46 & $30.67 \%$ & 52 & $34.67 \%$ & 77 & $51.33 \%$ & 175 & $38.89 \%$ \\
\hline
\end{tabular}

${ }^{a}$ This table represents score percentages for individual subscales. Scoring for this method is as follows: To meet the criteria for a core subscale, an individual must endorse at least one item on the intrusion subscale, or at least three items on the avoidance subscale, or at least two items on the arousal subscale. To be considered diagnostic, all three subscales must be endorsed. Bride (2007) noted that a symptom is considered present if the corresponding STSS item is rated 3, 4, or 5 (that is, occasionally, often, or very often). 
Overall, the intrusion scale is the most resonant for this sample of teachers. In all three opioid zones (low, medium, and high), over $80 \%$ of teachers screen for the presence of the intrusion symptoms of PTSD on the STSS. Even though the percentages of each subscale are high (all over $50 \%$ except for avoidance in the medium opioid zone), it is notable that the diagnostic level for all respondents of STSS using the algorithm method is $38.89 \%$. This low percentage is due to the instructions for the scoring method. The algorithm method is useful for scoring the subscales; it was "used to screen for the presence of PTSD due to secondary exposure" (Bride et al., 2007, p. 160). The scoring instructions require that all three subscales be endorsed for STS to be confirmed. So while each subscale is scored independently, and one teacher might endorse two different subscales, a diagnostic level of STS is not demonstrated unless all three are endorsed.

Comparison of all three scoring methods. All three scoring methods are useful in different ways. The most popular method for whole-test scoring is the summing/percentile method. It provides the most granularity and is seen most often in the literature. However, for ease of use, the cutoff method is recommended. The cutoff of 38 is at the low end of the moderate range and might indicate that a respondent seeks more information or intervention (Bride, 2007). The algorithm method helps identify the subscales with more clarity.

Comparatively, the summing/percentile method shows $59.56 \%$ of teachers $(\mathrm{N}=450)$ at the moderate level of STSS or above (see Table 4-23). Using the cutoff method, the percentage of teachers is the same for a moderate level or above: $59.56 \%$. The moderate level cutoff is 38 on both methods. Using the algorithm method, however, the percentage of teachers $(\mathrm{N}=450)$ endorsing a diagnostic level of STS is $38.89 \%$. This is a significant decrease. The decrease is due 
to the difference in scoring methodology and possibly gives a false negative because all three of the subscales must be endorsed for the algorithm method.

Table 4-23: Number and Percentage of Teachers at Each Level of the STSS (Summing/Percentile Method)

\begin{tabular}{|l|c|c|c|c|c|}
\hline & Little to no & Mild & Moderate & High & Severe \\
\hline & Score 17-27 & $\begin{array}{c}\text { Score 28- } \\
\mathbf{3 7}\end{array}$ & $\begin{array}{c}\text { Score 38- } \\
\mathbf{4 3}\end{array}$ & $\begin{array}{c}\text { Score 44- } \\
\mathbf{4 8}\end{array}$ & $\begin{array}{c}\text { Score 49 } \\
\text { or above }\end{array}$ \\
\hline STSS Level & $\mathbf{N}(\boldsymbol{\%})$ & $\mathbf{N}(\boldsymbol{\%})$ & $\mathbf{N}(\boldsymbol{\%})$ & $\mathbf{N}(\boldsymbol{\%})$ & $\mathbf{N}(\boldsymbol{\%})$ \\
\hline Low & $32(21.33)$ & $28(18.67)$ & $19(12.67)$ & $19(12.67)$ & $52(34.67)$ \\
\hline Medium & $28(18.67)$ & $38(25.33)$ & $21(14.00)$ & $23(15.33)$ & $40(26.67)$ \\
\hline High & $25(16.67)$ & $31(20.67)$ & $23(15.33)$ & $15(10.00)$ & $56(37.33)$ \\
\hline Total & $85(18.89)$ & $97(21.56)$ & $63(14.00)$ & $57(12.67)$ & $148(32.89)$ \\
\hline
\end{tabular}

In conclusion, the descriptive statistics addressed the first research question: What are $K$ 12 teachers' self-reported levels of secondary traumatic stress? Over half of the teachers in the study experienced STS at a moderate or greater level. The intrusion scale was the most resonant, and the teachers in the high opioid zone were most impacted. The sample, while not representative, was similar to the national population of teachers - although the sample exhibited a wider range of diversity.

\subsection{Measure of Internal Consistency}

Cronbach's alpha was calculated to determine the measure of internal consistency of the STSS to examine if the questions were assessing for the same construct - secondary traumatic stress. Like PTSD, STS is a construct that has three expressions-intrusion, avoidance, and arousal. Each of these was also measured using Cronbach's alpha (Table 4-24). 
Table 4-24: Results of the Cronbach Alpha for Total Study Population by STSS Subscale

\begin{tabular}{|l|c|c|c|c|}
\hline \multicolumn{1}{|c|}{ STSS } & $\boldsymbol{N}$ & $\boldsymbol{\alpha}$ & $\begin{array}{c}\text { Bride et al. (2004) } \boldsymbol{\alpha} \\
\boldsymbol{N = \mathbf { 2 8 7 }}\end{array}$ & Interpretation \\
\hline STSS Total Score & 450 & 0.95 & 0.93 & Excellent \\
\hline Avoidance Subscale & 450 & 0.89 & 0.87 & Good \\
\hline Arousal Subscale & 450 & 0.86 & 0.83 & Good \\
\hline Intrusion Subscale & 450 & 0.81 & 0.80 & Good \\
\hline
\end{tabular}

Cronbach's alpha was calculated at 0.95 for the total STSS $(\mathrm{N}=450)$ for this study. The interpretation of this coefficient is that the STSS shows excellent internal reliability (see Table 425). Although each of the subscales had lower alphas, they still showed good internal consistency. The whole STSS is a 17-item scale, whereas the subscales are only 5-7 questions each. The subscales, however, are specific components of a single, general construct (secondary stress); therefore, the whole scale score is meaningful. It is justified to measure alpha on the three subscales because they each are part of the entire STS construct. For this study, all the measures of alpha for the full STSS are comparable to Bride et al., (2004) and within an acceptable range for internal consistency.

Table 4-25: Results of the Cronbach Alpha by Opioid Zone for Total STSS

\begin{tabular}{|l|c|c|c|}
\hline \multicolumn{1}{|c|}{ Opioid Zone } & $\boldsymbol{N}$ & $\boldsymbol{\alpha}$ & Interpretation $^{-1}$ \\
\hline Low & 150 & 0.94 & Excellent \\
\hline Medium & 150 & 0.94 & Excellent \\
\hline High & 150 & 0.96 & Excellent $^{\mathrm{a}}$ \\
\hline Total (All three zones) & 450 & 0.95 & Excellent $^{2}$ \\
\hline
\end{tabular}

a This percentage might usually indicate redundancy on this subscale; however, the other reports of alpha are within range for internal consistency. Therefore, this might represent an issue in the opioid zone that requires further examination for the use of the STSS.

Considering each of the opioid zones as a specific sample, the calculation of Cronbach's alpha showed excellent internal consistency across the subset samples (see Table 4-26). This result makes sense, as this is just a more granular reporting of the total STSS alpha. 
In Table 4-26, each of the subscales is considered individually within each sample subset per opioid zone. This is regarded as a cross-scale report (Taber, 2017). The Cronbach alpha is lower for each subscale, in the subset sample than for the whole STSS. Because Cronbach alpha reports internal consistency for single constructs, it might be less useful for the cross-scale report.

Table 4-26: Results of Cronbach Alpha for Subscales by Zone

\begin{tabular}{|l|c|c|c|}
\hline STSS Subscale by Zone & $\boldsymbol{N}$ & $\boldsymbol{\alpha}$ & Interpretation \\
\hline Low & 150 & & \\
Avoidance & & 0.89 & Good \\
Arousal & & 0.86 & Good \\
Intrusion & & 0.79 & Acceptable \\
\hline Medium & 150 & & \\
Avoidance & & 0.88 & Good \\
Arousal & & 0.85 & Good \\
Intrusion & & 0.78 & Acceptable \\
\hline High & 150 & & \\
Avoidance & & 0.91 & Excellent \\
Arousal & & 0.88 & Good \\
Intrusion & & 0.85 & Good \\
\hline
\end{tabular}

What is also notable about the alphas reported in Table 4-26 is the intrusion scale. It might be possible that the STSS questions are more tailored for avoidance and arousal aspects of secondary stress. It is also possible that for this sample of respondents (teachers, as opposed to social workers for whom the STSS was validated), the avoidance and arousal scales are a more consistent measure of the construct of secondary stress than intrusion. It should be noted, though, that even the lowest alpha $(\alpha=0.78)$ is acceptable.

All of the Cronbach alpha calculations demonstrate that the STSS is a fitting test for the research questions and purpose of the dissertation. 


\subsection{Correlational Analysis}

Correlational measures were used to show whether there was a relationship between teachers' STS and the opioid impact area where they worked. Correlations were also used to determine whether the demographic variables, such as gender and age, were related to the levels of teacher STS. Spearman's rank correlation coefficient (Spearman's Rho) was used to examine these relationships and to examine whether one variable either increased or decreased in correlation with the other (McDonald, 2014). The variables of opioid zone along with demographic indicators of age, gender, race/ethnicity, and relationship status were first ranked, using the Excel ranking function to order the variables from greatest to smallest. The scores were then assigned a rank, with the rank " 1 " being assigned to the highest value, " 2 " to the next highest, etc. Using the Spearman's formula in Excel, the correlation was applied to the rankings to determine if there were significant correlations among the independent variables of opioid zones (low, medium, and high), age, gender, race/ethnicity, and relationship status and the dependent variables of teacher STSS scores and the STSS subscale scores of intrusion, arousal, and avoidance. The output number from the correlation of the paired data reflects a statistical measure of the strength of the relationship between the paired variables.

The correlational analysis will enable a determination of the strength and direction of the relationship. A correlation coefficient range between -1.00 and +1.00 will be considered to determine the strength of a relationship. A correlation of -1.00 or +1.00 is a perfect correlation, which means that the items perfectly relate to each other (Akoglu, 2018). A perfect correlation implies that the change in one variable's value is exactly proportional to the change in the other variable's value. However, a correlation coefficient between \pm 0.1 and 0 is so weak as to be negligent (Akoglu, 2018). Determining the coefficient's size, whether positive or negative, 
indicates the strength of the relationship. Consulting an interpretation table, like Akoglu's (2018) Spearman's Rho correlation coefficient interpretation, can assist with interpreting the strength of the relationship (see Figure 1, Chapter 3). In addition to determining the strength of the relationship, the direction of the correlation is also necessary to determine. This direction can be positive: the independent and dependent variables move in the same direction, either up or down. Conversely, the direction could be negative; this means that the independent and dependent variables will move in opposite directions.

In summary, because correlational measures can show whether variables are related to one another (Urdan, 2017), they are useful for examining relationships. Spearman's rank correlation coefficient (Spearman's Rho) is a nonparametric correlational analysis used to measure the strength of a relationship between paired data, with the assumption that as one variable increases, the other variable either increases or decreases (McDonald, 2014) and was, therefore, used to examine if there were significant correlations among the independent variables of opioid zones (low, medium, and high), age, gender, race/ethnicity, and relationship status and the dependent variables of teacher STSS scores and the STSS subscale scores of intrusion, arousal, and avoidance.

First, Table 4-27 shows the results of Spearman's rank correlation of opioid zone and teacher STSS score. In a Spearman's Rho analysis, the low, medium, and high opioid zones become a variable (all zones), but this does not mean that the differences between the zones were lost. The zones were ranked, as were the subscale scores and total STSS scores. Then the correlation was applied to the rankings to determine if there were significant correlations among the independent variables of opioid zones (low, medium, and high) and the dependent variables of teacher STSS scores and the STSS subscale scores of intrusion, arousal, and avoidance. The 
output numbers from the correlation of the paired data (see Table 4-27) reflects the statistical measure of the strength of the relationship between these paired variables. This table shows that there was no significant difference in total or subscale STSS scores between the low, medium, and high opioid impact zones. In other words, there is no relationship between the STSS score or subscale and the opioid epidemic zone.

Table 4-27: Spearman's Rho by Opioid Zone $(\mathrm{N}=450)$

\begin{tabular}{|c|c|c|c|c|}
\hline $\begin{array}{c}\text { Opioid } \\
\text { Epidemic } \\
\text { Zone }\end{array}$ & $\begin{array}{c}\text { STSS Instrument } \\
\text { Total }\end{array}$ & $\begin{array}{c}\text { STSS Subscale } \\
\text { Avoidance }\end{array}$ & $\begin{array}{c}\text { STSS Subscale } \\
\text { Intrusion }\end{array}$ & $\begin{array}{c}\text { STSS Subscale } \\
\text { Arousal }\end{array}$ \\
\hline $\begin{array}{c}\text { All zones } \\
\text { (Low, Med., } \\
\text { High) }\end{array}$ & .029 & .026 & .027 & .034 \\
\hline
\end{tabular}

The values listed in Table 4-27 were "weak," "negligible," "poor," to "none” per Akoglu (2018), as the highest positive correlation was .034 , and the weakest positive correlation was .026. There are no negative correlations. Because a value of -1 would indicate a negative correlation between the data sets, called the ranks, and a value of +1 would indicate a positive correlation, it is clear that there is not a correlation between the opioid zones and the STS scores on the STSS. This was the case for both the total score (listed in the first column) and the subscales (listed in the subsequent columns).

Next, Table 4-28 shows the results of Spearman's Rho with the demographic variables of age, gender, race, and relationship status for the whole sample $(\mathrm{N}=450)$. 
Table 4-28: Spearman's Rho for Teachers $(\mathrm{N}=450)$

\begin{tabular}{|l|c|c|c|c|}
\hline \multicolumn{1}{|c|}{ Variable } & $\begin{array}{c}\text { STSS Subscale } \\
\text { Avoidance }\end{array}$ & $\begin{array}{c}\text { STSS Subscale } \\
\text { Intrusion }\end{array}$ & $\begin{array}{c}\text { STSS Subscale } \\
\text { Arousal }\end{array}$ & $\begin{array}{c}\text { STSS Instrument } \\
\text { Total }\end{array}$ \\
\hline Age & -.061 & -.077 & -.091 & -.081 \\
\hline Gender & .089 & .036 & .036 & .070 \\
\hline Race & -.072 & -.114 & -.020 & -.072 \\
\hline Marital Status & .016 & -.048 & .008 & -.003 \\
\hline
\end{tabular}

The values listed in Table 4-28 were "weak," "negligible," "poor," to "none" per Akoglu (2018), as the highest positive correlation was .089, and the lowest positive correlation was .008. The greatest negative correlation was -.114, and the weakest negative correlation was -.003.

Because a value of -1 would indicate a negative correlation between the data sets, called the ranks, and a value of +1 would indicate a positive correlation, it is clear that there is not a correlation between the demographic indicators and the STS scores on the STSS.

The sample of teachers for the study had some diversity concerning gender and race/ethnicity in the different opioid zones. Spearman's Rho correlation was rerun for each of the opioid zones to see greater detail in the associations. Although the correlations are still weak, a greater range was seen (see Table 4-29): the highest positive correlation was .108 (compared to .089 for the total population above), and the lowest positive correlation was .005 (compared to .008). The greatest negative correlation was -.191 (compared to -.114), and the weakest negative correlation was -.003 (the same as above). By disaggregating the data, a more nuanced picture is revealed, but the associations are still weak or virtually non-existent. Teacher STS is not related to the demographic characteristics of teachers. 
Table 4-29: Spearman's Rho Comparison of Low-Medium-High Zone Correlations to Total Correlations

\begin{tabular}{|c|c|c|c|c|c|}
\hline Variable & $\begin{array}{c}\text { STSS: } \\
\text { Total Scale } \\
\text { and Subscale }\end{array}$ & $\begin{array}{c}\text { Spearman's } \\
\text { Rho for } \\
\text { Low Opioid } \\
\text { Epidemic } \\
\text { Zone } \\
\text { (n = 150) }\end{array}$ & $\begin{array}{c}\text { Spearman's } \\
\text { Rho for } \\
\text { Medium } \\
\text { Opioid } \\
\text { Epidemic } \\
\text { Zone } \\
(\mathbf{n}=150)\end{array}$ & $\begin{array}{l}\text { Spearman's } \\
\text { Rho for } \\
\text { High Opioid } \\
\text { Epidemic } \\
\text { Zone } \\
\text { (n= 150) }\end{array}$ & $\begin{array}{c}\text { Spearman's } \\
\text { Rho for } \\
\text { Total All } \\
\text { Zones } \\
\\
(\mathbf{N}=\mathbf{4 5 0})\end{array}$ \\
\hline Age & Total STSS & -0.069 & -0.059 & -0.098 & -0.081 \\
\hline Age & Avoidance & -0.019 & -0.050 & -0.092 & -0.061 \\
\hline Age & Intrusion & -0.104 & -0.083 & -0.040 & -0.077 \\
\hline Age & Arousal & -0.092 & -0.042 & -0.129 & -0.091 \\
\hline Gender & Total STSS & 0.086 & 0.031 & 0.083 & 0.070 \\
\hline Gender & Avoidance & 0.106 & 0.077 & 0.081 & 0.089 \\
\hline Gender & Intrusion & 0.013 & -0.009 & 0.080 & 0.036 \\
\hline Gender & Arousal & 0.108 & -0.006 & 0.083 & 0.036 \\
\hline Race & Total STSS & -0.123 & -0.054 & -0.019 & -0.072 \\
\hline Race & Avoidance & -0.095 & -0.058 & -0.032 & -0.072 \\
\hline Race & Intrusion & -0.191 & -0.106 & -0.041 & -0.114 \\
\hline Race & Arousal & -0.078 & 0.005 & 0.012 & -0.020 \\
\hline $\begin{array}{l}\text { Marital } \\
\text { Status }\end{array}$ & Total STSS & -0.009 & 0.061 & -0.054 & -0.003 \\
\hline $\begin{array}{l}\text { Marital } \\
\text { Status }\end{array}$ & Avoidance & -0.008 & 0.092 & -0.026 & 0.016 \\
\hline $\begin{array}{l}\text { Marital } \\
\text { Status }\end{array}$ & Intrusion & -0.046 & 0.040 & -0.124 & -0.048 \\
\hline $\begin{array}{l}\text { Marital } \\
\text { Status }\end{array}$ & Arousal & 0.014 & 0.032 & -0.023 & 0.008 \\
\hline
\end{tabular}

\subsection{Conclusion}

The purpose of this study was to investigate if K-12 teachers' self-reported level of secondary traumatic stress (STS) relates to the opioid mortality level in their communities. This chapter presented the results of the survey and the data analysis through descriptive statistics and 
correlational analysis. In concluding the chapter, it is helpful to review how this data analysis answers each of the main research questions.

\subsubsection{Research Question Summary}

RQ1: What are K-12 teachers' self-reported levels of secondary traumatic stress? Two avenues were pursued to answer this question. First, descriptive statistics profiled the sample to identify the qualities of the participants, including how the participants described the adverse experiences of students in their classes. Second, the level of STS in teachers was examined by the whole score, by subscale, and by combined subscale score.

Teacher demographics. Although a statement cannot be made as to the generalizability of this study to the population of teachers nationally, the demographics of teachers in this study (N $=450$ ), from 25 states and Washington, D.C., in all three opioid epidemic zones, is similar to teachers nationally. Between 91-93\% of all teachers surveyed endorsed the types of adverse events experienced by their students, thereby supporting the possible construct of teacher STS.

Teacher STS. The total mean score for participants $(\mathrm{N}=450)$ on the STSS was 42.06 (SD $=14.79)$. The lowest mean was in the medium opioid zone $(M=40.23, S D=13.44)$, with the highest score in the high opioid epidemic zone $(M=43.78, S D=16.00)$. Scores from the low opioid zone were $M=42.16, S D=14.71$. All of these scores, from every opioid zone, are at the moderate level. By using the cutoff method of interpretation, $59.56 \%$ of teachers scored 38 or higher on the STSS, which is an indicator that interventions are therapeutically indicated for STS (Bride, 2007; Bride et al., 2007).

On the intrusion subscale, the total percentile score $(M=12.93, \mathrm{SD}=4.51)$ is at the high/severe level. Using the algorithm-interpretation method, $88.62 \%$ of teachers endorsed intrusion at a diagnostic level, 64.67\% endorsed arousal symptoms, and 50.89\% endorsed 
avoidance. The highest scores noted, for all three zones, were in the area of intrusion symptoms $(82.67 \%-88.00 \%)$.

Over half of the teachers in the study $(59.56 \%)$ experienced STS at a moderate or greater level. The intrusion scale was the most resonant; looking at the individual questions, the three questions that reflected this finding were intrusive thoughts about students $(71.8 \%)$, physiological symptoms when thinking about work (54.6\%), and reliving student trauma (54.2\%). The teachers in the high opioid zone were most impacted by full STSS score $(M=$ $43.78, \mathrm{SD}=16.00)$, with $62.67 \%$ in the high opioid zone scoring at 38 or higher. The sample, while not representative, was similar to the national population of teachers, but generalizability cannot be claimed.

\section{RQ2: Is there a relationship between teachers' self-reported levels of secondary} traumatic stress when teachers are from states of high-opioid impact, states of medium-opioid impact, and states of low-opioid impact? Correlational measures were used in the study to show whether there was a relationship between teachers' STS and their opioid impact area. Spearman's rank correlation coefficient (Spearman's Rho) was used to examine this relationship and to examine whether one variable either increased or decreased in correlation with the other (McDonald, 2014). The teachers' reported STS level, as determined by their STSS scores on the whole test correlated with the variable of opioid epidemic zone at $\rho=.029$, which is negligible or none and was non-significant. This is finding for the total score test, and it means that STS on the total score is not related to whether a teacher is in the low, medium, or high zone. The results of the subscale tests, as a reflection of the teachers' self-reported levels of STS and opioid zone, were correlated at $\rho=.026$ (avoidance scale), $\rho=.027$ (intrusion scale), and $\rho=.034$ (arousal scale), which all demonstrate negligible or no correlation, also reflecting non-significance. There 
is no relationship between teachers' self-reported levels of secondary traumatic stress when teachers are from states of high-opioid impact, states of medium-opioid impact, and states of low-opioid impact.

RQ3: Is there a relationship between self-reported levels of secondary traumatic stress and the characteristics of teachers? Correlational measures were used in the study to show whether there was a relationship between teachers' STS and the demographic identifiers of teachers, particularly age, gender, race/ethnicity, and relationship status. Spearman's rank correlation coefficient (Spearman's Rho) was used to examine this relationship and to examine whether one variable either increased or decreased in correlation with the other (McDonald, 2014).

RQ3a: Is there a relationship between self-reported levels of secondary traumatic stress and the age of teachers? The teachers' reported STS level, as determined by their STSS scores on the whole test correlated with the variable of age was determined to be $\rho=-.081$, which is negligible or none and was non-significant.

$R Q 3 b:$ Is there a relationship between self-reported levels of secondary traumatic stress and gender? The teachers' reported STS level, as determined by their STSS scores on the whole test correlated with the variable of gender, was determined to be $\rho=.070$, which is negligible or none and was non-significant.

$R Q 3 c:$ Is there a relationship between self-reported levels of secondary traumatic stress and racelethnicity? The teachers' reported STS level, as determined by their STSS scores on the whole test correlated with the variable of race/ethnicity, was determined to be $\rho=-.072$, which is negligible or none and was non-significant. 
RQ3d: Is there a relationship between self-reported levels of secondary traumatic stress and relationship status? The teachers' reported STS level, as determined by their STSS scores on the whole test correlated with the variable of relationship status, was determined to be $\rho=$ .003 , which is negligible or none and was non-significant.

All of the demographic variables demonstrated a negligible or no correlation, which means that no significance was found; therefore, there is not a relationship between self-reported levels of secondary traumatic stress and the characteristics of teachers.

In the final analysis, teachers in the study have a moderate level of STS, regardless of where they live, and regardless of their demographic identity. Intrusion symptoms appear to be the most impactful on teachers, regardless of the zone. The above results will be discussed further in the next chapter. 


\section{Chapter Five: Discussion}

The purpose of this study was to understand better the prevalence of secondary traumatic stress (STS) in teachers and to determine if this prevalence has any relationship to the opioid mortality rate in communities. An additional purpose was to investigate the relationship between STS and teacher characteristics such as age, gender, race/ethnicity, age, and relationship status. Data were collected through a 34-item anonymous survey of 450 teachers from 26 states and Washington, D.C. The three main research questions and four sub-questions were analyzed with descriptive statistics and Spearman's Rho correlation.

\subsection{Findings of the Study}

Research tells a story. What started as a few media accounts from overwhelmed teachers in precarious communities has begun to take shape through continued and persistent storytelling. This dissertation is now part of that story, adding line and measure in areas of need. The findings in this section are organized first around the three research questions, which coalesce broadly around these two STS topics: prevalence of teacher-reported STS and relationship of teacherreported STS to teacher characteristics. Next, the implications of the findings are discussed through the salient construct of STS. Limitations are reviewed, and directions for future research are considered. While the research questions are thoroughly addressed, the participants also speak to help a picture take shape.

\subsubsection{Prevalence of Teacher-Reported STS}

From the literature before the study, it was clear that there was very little information on secondary traumatic stress, specifically on teachers. This lack of existing research means there is no way to track if the prevalence of teacher STS is increasing or decreasing. In some ways, STS 
appears to be hidden stress - teachers have it, experience it, but do not have the vocabulary or construct to discuss it.

To develop policies, programs of professional development, and the types of supports needed by teachers with STS, the prevalence of teacher STS should be determined. The first research question sought to do this, and the size of the sample population $(\mathrm{N}=450)$, with the number of states (25 plus Washington, D.C.), was an attempt to survey enough teachers, broadly, so that the conversation about STS was less local and more national.

\section{Research Question 1: What are K-12 teachers' self-reported levels of secondary}

traumatic stress? Using the three different ways to score the STSS presents the researcher with more data than only a mean score for the participants. A mosaic of prevalence data appears from the various methods.

STSS full score interpretation and comparisons. The teachers in this study self-reported moderate to high levels of secondary traumatic stress $(M=42.06, S D=14.79)$. The STSS whole test mean score was interpreted using the percentile interpretation method (Bride et al., 2007). This mean score of 42.06 falls between the $75^{\text {th }}$ percentile, which is moderate (37.00), and the $90^{\text {th }}$ percentile, which is high (43.80). The mean score of 42.60 is in the high-moderate range. Compared to other studies utilizing the STSS, this is an elevated score. Also, this is a large standard deviation, which reflects that there is a wide distribution of scores among individual teachers in the sample. The STSS measures secondary traumatic stress, the type of indirect stress that results from helping or wanting to help a traumatized person. The teachers who responded to the STSS produced a mean score of 42.60 . This mean is 21.80 points higher than the mean score reported by teachers in a school identified as an environment of low or little stress (Denham, 2018). The standard deviation for this full score is 14.79 , which reflects that the highest score 
was 57.39, in the severe range. Even compared to the high-stress job of forensic interviewing, the teacher STSS mean is 8.40 points higher. The STSS full-scale test revealed a high score for teachers nationally.

Table 5-1 ${ }^{\text {a }}$ : Comparison of STS Study Mean for Full STSS Score

\begin{tabular}{|c|c|c|}
\hline Study & $\begin{array}{l}\text { Population } \\
\text { Sample Size }\end{array}$ & $\begin{array}{c}\text { Full STSS } \\
\text { Score }^{\mathrm{a}} \\
\text { Mean }\end{array}$ \\
\hline Denham (2018) & $\begin{array}{l}\text { High school teachers, no school decay } \\
\qquad N=84\end{array}$ & 20.80 \\
\hline McLennan et al. (2016) & $\begin{array}{c}\text { Post-disaster field research } \\
\text { interviewers } \\
\mathrm{N}=33\end{array}$ & 26.42 \\
\hline Beckmann (2015) & $\begin{array}{l}\text { Disaster Mental Health Volunteers } \\
\qquad \mathrm{N}=92\end{array}$ & 28.34 \\
\hline Bride (2007) & $\begin{array}{l}\text { Social workers } \\
\qquad N=282\end{array}$ & 29.69 \\
\hline Mordeno et al. (2017) & $\begin{array}{l}\text { Nurses (Philippines) } \\
\qquad \mathrm{N}=241\end{array}$ & 30.38 \\
\hline Bride et al. (2009) & $\begin{array}{l}\text { Substance Abuse Counselors } \\
\qquad \mathrm{N}=225\end{array}$ & 31.20 \\
\hline Ting et al. (2005) & $\begin{array}{l}\text { Social workers } \\
\quad \mathrm{N}=275\end{array}$ & 33.30 \\
\hline Denham (2018) & $\begin{array}{l}\text { High school teachers, school decay } \\
\qquad N=88\end{array}$ & 34.03 \\
\hline Perron \& Hiltz (2006) & $\begin{array}{l}\text { Forensic interviewers of abused } \\
\text { children } \\
\mathrm{N}=66\end{array}$ & 34.20 \\
\hline Burr et al. (2020) & $\begin{array}{c}\text { Respiratory therapists } \\
\text { (for past } 12 \text { months) } \\
\mathrm{N}=201\end{array}$ & 35.89 \\
\hline $\begin{array}{l}\text { Akinsulure-Smith et al. } \\
\text { (2018) }\end{array}$ & $\begin{array}{l}\text { Refugee Resettlement Workers } \\
\qquad \mathrm{N}=210\end{array}$ & 36.67 \\
\hline Burr et al. (2020) & $\begin{array}{l}\text { Respiratory therapists } \\
\text { (for past } 30 \text { days) } \\
\mathrm{N}=201\end{array}$ & 36.98 \\
\hline
\end{tabular}




\begin{tabular}{|l|c|c|}
\hline Morrison \& Joy (2016) & $\begin{array}{c}\text { Emergency Nurses (Scotland) } \\
\mathrm{N}=80\end{array}$ & 37.40 \\
\hline $\begin{array}{l}\text { Smith Hatcher et al. } \\
(2011)\end{array}$ & $\begin{array}{c}\text { Juvenile justice teachers and staff } \\
\mathrm{N}=118\end{array}$ & 37.74 \\
\hline $\begin{array}{l}\text { Ben-Porat \& Itzhaky } \\
(2011)\end{array}$ & $\begin{array}{c}\text { Domestic Violence Therapists } \\
\text { Received training } \\
\mathrm{N}=103\end{array}$ & 38.42 \\
\hline $\begin{array}{l}\text { Ben-Porat \& Itzhaky } \\
(2011)\end{array}$ & $\begin{array}{r}\text { Domestic Violence Therapists } \\
\text { Did not receive training } \\
\mathrm{N}=40\end{array}$ & 41.48 \\
\hline $\begin{array}{l}\text { STS Teacher Study } \\
\text { (2020) }\end{array}$ & $\begin{array}{r}\text { K-12 Public School Teachers } \\
\mathrm{N}=450\end{array}$ & $\mathbf{4 2 . 6 0}$ \\
\hline
\end{tabular}

${ }^{a}$ Scores less than 28 points are below the 50th percentile: no STS. Scores between $28-37$ points are between 51 st to the 75th percentile: mild STS. Scores between $38-43$ points are between the 76th to the 90th percentile: moderate STS. Scores between $44-48$ points are between the 91 st to the 95 th percentile: high STS. Scores at 49 points or above are at or above the 95th percentile: severe STS.

Comparatively, using the percentile interpretation method, teachers in this study show a high level of stress. To evaluate how teachers in this study scored at this high percentile range, I will revisit the frequencies for teachers in each of the opioid epidemic zones, especially at the high/severe range.

In the table below, the comparison of the percentage of teachers at each opioid epidemic level shows the preponderance of teachers who self-reported STSS scores in the high/severe range.

Table 5-2: Number and Percentage of Teachers at Each Level of the STSS (Summing/Percentile Method)

\begin{tabular}{|l|c|c|c|c|c|}
\hline STSS Level & Little to no & Mild & Moderate & High & Severe \\
\hline & $\begin{array}{c}\text { Score 17- } \\
\mathbf{2 7}\end{array}$ & $\begin{array}{c}\text { Score 28- } \\
\mathbf{3 7}\end{array}$ & $\begin{array}{c}\text { Score 38- } \\
\mathbf{4 3}\end{array}$ & $\begin{array}{c}\text { Score 44- } \\
\mathbf{4 8}\end{array}$ & $\begin{array}{c}\text { Score 49 } \\
\text { or above }\end{array}$ \\
\hline & $\mathbf{N}(\boldsymbol{\%})$ & $\mathbf{N}(\boldsymbol{\%})$ & $\mathbf{N}(\boldsymbol{\%})$ & $\mathbf{N}(\boldsymbol{\%})$ & $\mathbf{N}(\boldsymbol{\%})$ \\
\hline Low & $32(21.33)$ & $28(18.67)$ & $19(12.67)$ & $19(12.67)$ & $52(34.67)$ \\
\hline Medium & $28(18.67)$ & $38(25.33)$ & $21(14.00)$ & $23(15.33)$ & $40(26.67)$ \\
\hline High & $25(16.67)$ & $31(20.67)$ & $23(15.33)$ & $15(10.00)$ & $56(37.33)$ \\
\hline Total & $85(18.89)$ & $97(21.56)$ & $63(14.00)$ & $57(12.67)$ & $148(32.89)$ \\
\hline
\end{tabular}


In each opioid zone, the high/severe percentile is the highest percentage; however, the high opioid epidemic zone has the highest frequency of teachers reporting scores at this level.

Figure 5-1: Frequency of Teachers with STS in Each Opioid Zone

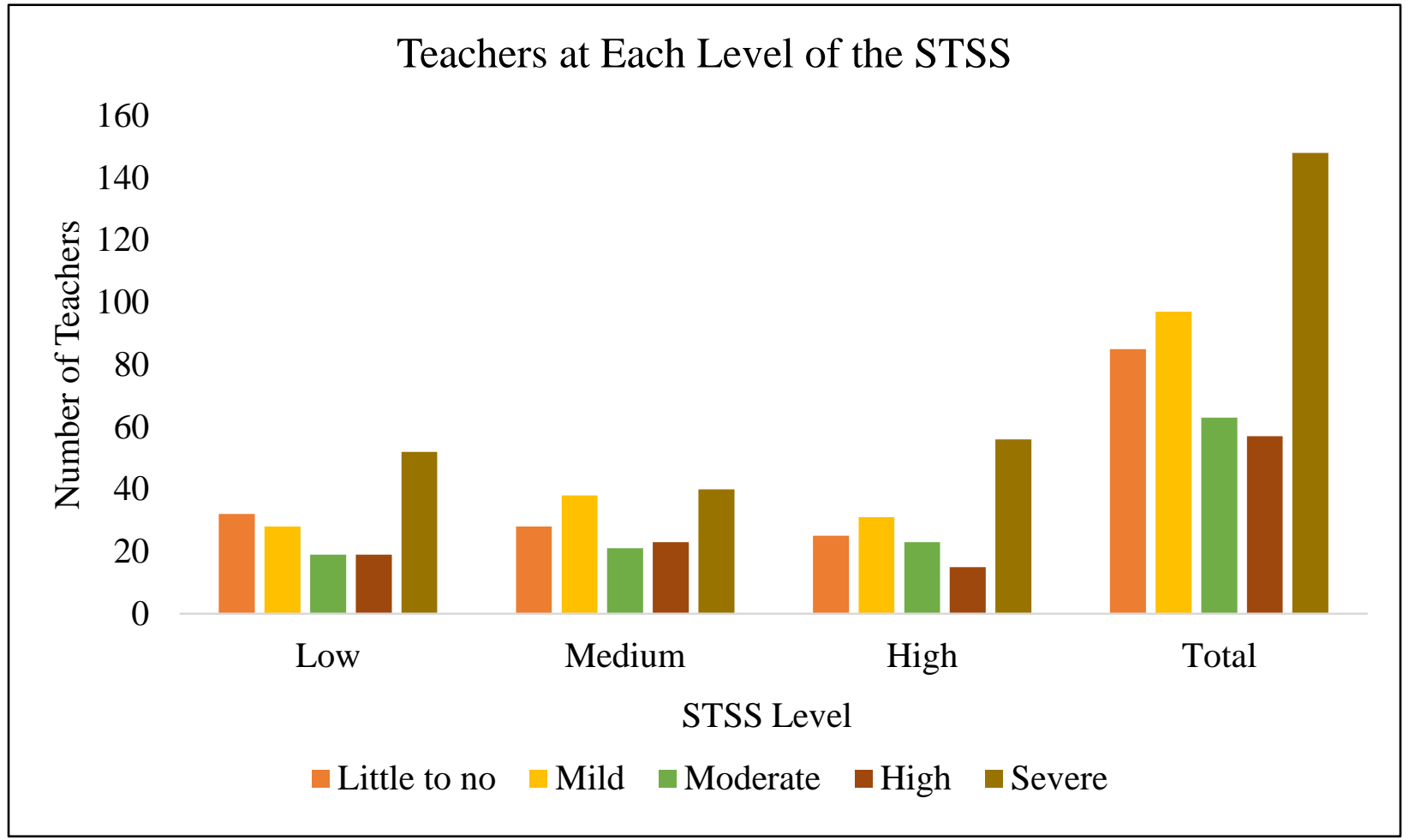

Possible explanations. Three potential issues were considered for higher self-reports of the teacher STS in this study: sample, survey, or STS construct issues. Sample considerations focused on the idea that maybe there were methodology issues with the sample of teachers. Survey issues explored questions of survey design and delivery. In the end, though, STS construct issues seemed like the explanation of best fit: K-12 public school teachers had an unusually high mean score for STS because many teachers have a high level of secondary traumatic stress. Each issue was evaluated to consider the integrity of the study findings.

Sample issues. With a high STS score, the sample and sampling procedure was reviewed. The sample was sufficiently large; comparatively, it was the largest of STSS studies that were located at the time of the dissertation. Several larger studies were found and examined (see Table 2-10), but these studies did not use the STSS (Bride et al., 2004), so they are non-comparable. 
Additionally, the sample consisted of K-12 teachers only. It is possible that this fact, this homogeneity of the sample, is one of the reasons that account for the higher STS scores. Many of the studies on school sites used blended populations of teachers, administrators, and other professionals (like school psychologists, speech pathologists, etc.). Denham (2018) surveyed teachers who self-selected into the study, through craigslist and Facebook and snowball sampling. Teachers in Denham's study who worked in schools experiencing disrepair scored significantly higher on the STSS (63.6\%) than teachers working in schools that were not classified as disrepair or blighted schools $(\mathrm{t}=-6.340, \mathrm{p}<.001, \mathrm{df}=170, \mathrm{~d}=.97)$. Denham's study lends support to the notion that teachers in communities of high stress might endorse a higher STSS score.

Further support for the sample as a homogeneous teacher sample comes from the demographic information for teachers, which lists their teaching assignments. The primary teaching assignments are listed in Figure 5-2. From the responses, $82 \%$ of teachers work in traditional K-12 general education classroom settings, $7 \%$ in special education, and $10 \%$ in alternative settings, including blended and multiple settings. Participants had to pass two gatekeeping processes in the survey: first, Qualtrics randomly selected from their pool of K-12 teachers in the states that were stipulated for this study. Second, to add an extra measure of safety for the participants and the study, a qualifying question asked about the profession and deselected any respondent who did not select teacher for the answer. While $1 \%$ of respondents preferred not to answer the question $(n=3)$ regarding teaching setting, to qualify for the survey, as much care as possible was taken to assure that the participants were teachers. 
Figure 5-2: Percent of Teachers in STS Study

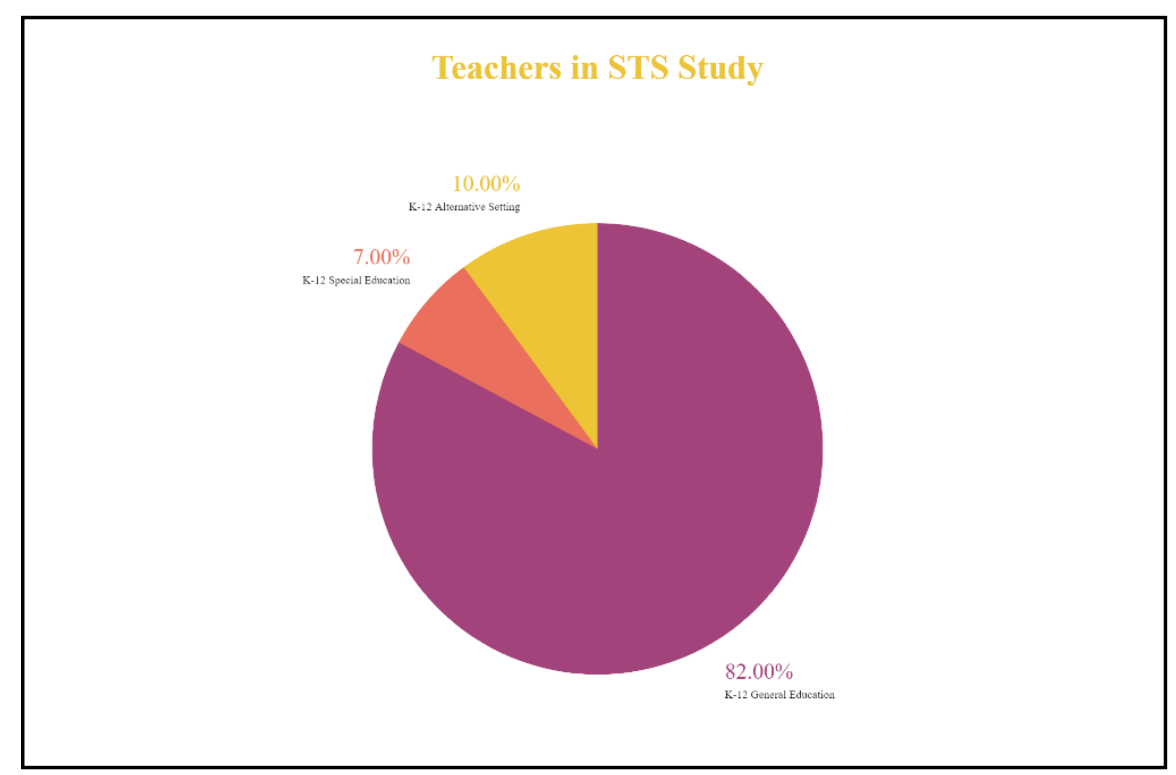

Survey issues. Since it was clear that the sample $(\mathrm{N}=450)$ was $\mathrm{K}-12$ public school teachers from across the United States, the next issue to explore to understand the high teacher STS score was the survey itself. First, regardless of survey design, there was the possibility that respondents had quickly answered the questions without considering the issue. Qualtrics used their metrics to certify and rate the quality of respondent interaction with the instrument. Using the Qualtrics rubric for quality rating, the respondents for the survey were rated at the highest level of quality. The highest level means that participants did not skip questions, took time to answer, did not select all of one choice for the multiple-choice questions, and appeared to give answers that performed within the logic frame necessary for Qualtrics' highest rating (of five scaled scores) for quality assessment.

The portion of the survey that was assessing STS was an instrument that has been used globally, the STSS (Bride et al., 2004). The validity of this survey was discussed at length in Chapter Three. Care was taken not to change any of the wording or formatting of the survey (other than using the occupational words that pertain to teachers, as is suggested by Bride et al., 
2004). It was important to have the STSS appear in its standard form so that the results of this study could be compared to the standardized results of other studies using the STSS.

Cronbach's alpha $(\alpha)$, also called coefficient alpha, was calculated as a measure of internal consistency or reliability (Cronbach, 1951) to examine if the questions assessed for the same construct—secondary traumatic stress. The results for this study were as consistent as Bride et al. (2004), as shown in Table 5-3 (reprinted from Chapter Four):

Table 5-3: Results of the Cronbach Alpha for Total Study Population by STSS Subscale

\begin{tabular}{|c|c|c|c|c|}
\hline STSS & $N$ & $\alpha$ & $\begin{array}{c}\text { Bride et al. (2004) } \alpha \\
N=\mathbf{2 8 7}\end{array}$ & Interpretation \\
\hline STSS Total Score & 450 & 0.95 & 0.93 & Excellent \\
\hline Avoidance Subscale & 450 & 0.89 & 0.87 & Good \\
\hline Arousal Subscale & 450 & 0.86 & 0.83 & Good \\
\hline Intrusion Subscale & 450 & 0.81 & 0.80 & Good \\
\hline
\end{tabular}

Finally, the voice of the participants supported the survey measure as a measure of stress:

- Colorado teacher: [Supporting children who experience significant adversity and trauma] "gave me depression and anxiety over all the years."

- California teacher: "Trying to help the kids take a toll on my stress."

- Pennsylvania teacher: "Sometimes I find myself internalizing my students' problems. I have to be careful not to get too depressed about the situations."

- California teacher: "It's difficult seeing your students go through harmful situations and often I want to help them. Sometimes the level of situations they are experiencing keeps me up due to worry about them."

- Alabama teacher: "I feel powerless to help."

Themes of stress, as expressed through helplessness, emotional reactivity, difficulty with managing life and teaching, references to past trauma, and prolonged worry, were in the majority. These themes of stress were universal in all three opioid zones, stated by teachers of all ages, race/ethnicities, and genders. 
Construct issues. Teachers in this study had a high level of STS. As will be discussed in the next section on research question two, this level of STS was not related to any of the demographic identifiers, like gender or race/ethnicity. It appears to be a finding of a homogeneous sample of K-12 public school teachers. The last area to consider, then, is the construct of STS itself: is secondary stress a viable construct for teachers? To answer this question, I look first at the body of research regarding teacher STS; then, I look at these particular teachers to determine if STS appears to be a construct that applies to these teachers.

Motta (2012) described the mechanism of STS for school personnel, including teachers, which involves the transfer of trauma-like symptoms from the traumatized to those affected by STS:

In general, the term secondary trauma refers to the experience of negative affective, cognitive, and behavioral states that result from extended and close contact with others who have been traumatized. School personnel who work extensively with traumatized children can also acquire secondary trauma reactions from the children. (p. 257) This mechanism is predicated on the supposition that children experience events that result in trauma and that they then, subsequently, attend school. The teacher participants in this study self-reported their perceptions of student trauma through the utilization of the PHL-ACEs categories (Health Federation of Philadelphia and Philadelphia ACE Research and Data Committee, 2012). The top five categories of teacher-reported issues are in Table 5-4. According to the teacher report, the potential for secondary traumatic stress is likely related to the amount of trauma found in teachers' students. These teachers endorsed high levels of ACESs among their students. 
Table 5-4: Top Five Categories of Teachers' Perceptions of Student Adverse Childhood Experiences (ACEs)

\begin{tabular}{|l|c|c|c|c|}
\hline \multicolumn{5}{|c|}{$\begin{array}{c}\text { Percent of teachers endorsing adverse childhood experiences } \\
\text { present in students' household }\end{array}$} \\
\hline \multicolumn{1}{|c|}{ ACEs Types } & Total \% & Low Zone \% & Medium Zone \% & High Zone \% \\
\hline $\begin{array}{l}\text { Emotional } \\
\text { Abuse }\end{array}$ & $61 \%$ & $58 \%$ & $61 \%$ & $65 \%$ \\
\hline $\begin{array}{l}\text { Experienced } \\
\text { Death in Family }\end{array}$ & $52 \%$ & $51 \%$ & $57 \%$ & $47 \%$ \\
\hline $\begin{array}{l}\text { Emotional } \\
\text { Neglect }\end{array}$ & $50 \%$ & $55 \%$ & $48 \%$ & $49 \%$ \\
\hline $\begin{array}{l}\text { Lived in Foster } \\
\text { Care }\end{array}$ & $48 \%$ & $49 \%$ & $53 \%$ & $42 \%$ \\
\hline $\begin{array}{l}\text { Household } \\
\text { Substance Abuse }\end{array}$ & $47 \%$ & $48 \%$ & $46 \%$ & $46 \%$ \\
\hline
\end{tabular}

Additional research supports the notion that STS is a construct that is viable for teachers. Robinson (2005) found that $33.15 \%$ of teachers were at risk for compassion fatigue, a construct like STS. Smith Hatcher et al. (2011) reported a full scale STSS score of 37.74 for educators and, more importantly, 39\% of these educators met all three core diagnostic criteria for posttraumatic stress disorder. Caringi et al. (2015) reported a mean of 39.00 for the full STSS for educators, with $75 \%$ exceeding subscale cut-offs on all three subscales. This research is a possible indication that the teachers might meet the diagnosis of PTSD if a standardized assessment for PTSD was administered (Caringi et al., 2015). The teachers in the STS teacher study endorsed PTSD at the diagnostic level at rates similar to or above other research findings in STS literature (see Figure 5-3). If a total score on the STSS is 38 or higher, then interventions are therapeutically indicated for STS (Bride, 2007; Bride et al., 2007). Likewise, if teachers answer a particular question with a score of 3,4 , or 5 , then the item is considered endorsed. For the intrusion scale (questions $2,3,6,10,13$ ), teachers only need to endorse at least one item; for the 
avoidance scale (questions 1, 5, 7, 9, 12,14), teachers must endorse three or more items; and for the arousal scale (questions $4,8,11,15,16$ ), two or more items must be answered at 3,4 , or 5 . For a diagnostic level of PTSD, however, all three of these endorsements must be met. In this study, on the STSS, over half of the teachers in the high opioid zone score on the STSS (see Figure 5-3) at a level that might meet the diagnosis of PTSD if given a standardized assessment for PTSD (Caringi et al., 2015).

Figure 5-3: PTSD Endorsement for Teachers in STS Study in Each Opioid Zone

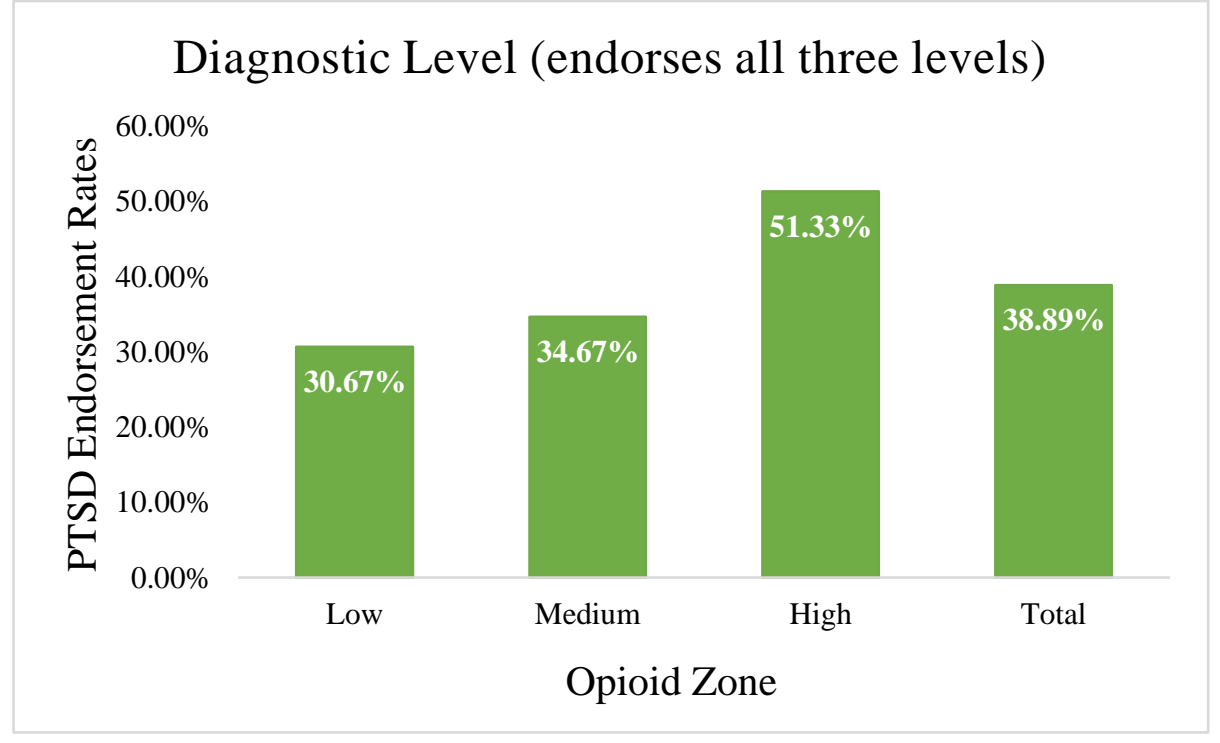

The finding that many teachers in this study have high levels of secondary stress is also reflected in their responses to the open-ended questions from the survey:

- New Hampshire teacher: "You can't stop thinking about what some of these kids have been through."

- Georgia teacher: "As a child, I also endured forms of abuse and trauma, so the most difficult aspect for me is thinking about the way I was hurt."

- Missouri teacher: [I find it difficult] "Continuing to teach academics when I know the trauma they are experiencing."

The findings for this study are comparatively high, but not outside the range of PTSD endorsement for professionals who have STS. In Figure 5-4, the teachers from this study are 
shown at $38.89 \%$, compared to $39 \%$ of educators in juvenile justice settings (Smith Hatcher et al., 2011) and emergency nurses (Morrison \& Joy, 2016). However, 38.89\% of teachers who endorsed a diagnostic level of PTSD for this study is higher than $15.2 \%$ of social workers (Bride, 2007) or $19 \%$ of substance abuse counselors (Bride, 2009).

Figure 5-4: Teacher STS Subscale PTSD Percentage Compared to Other Studies

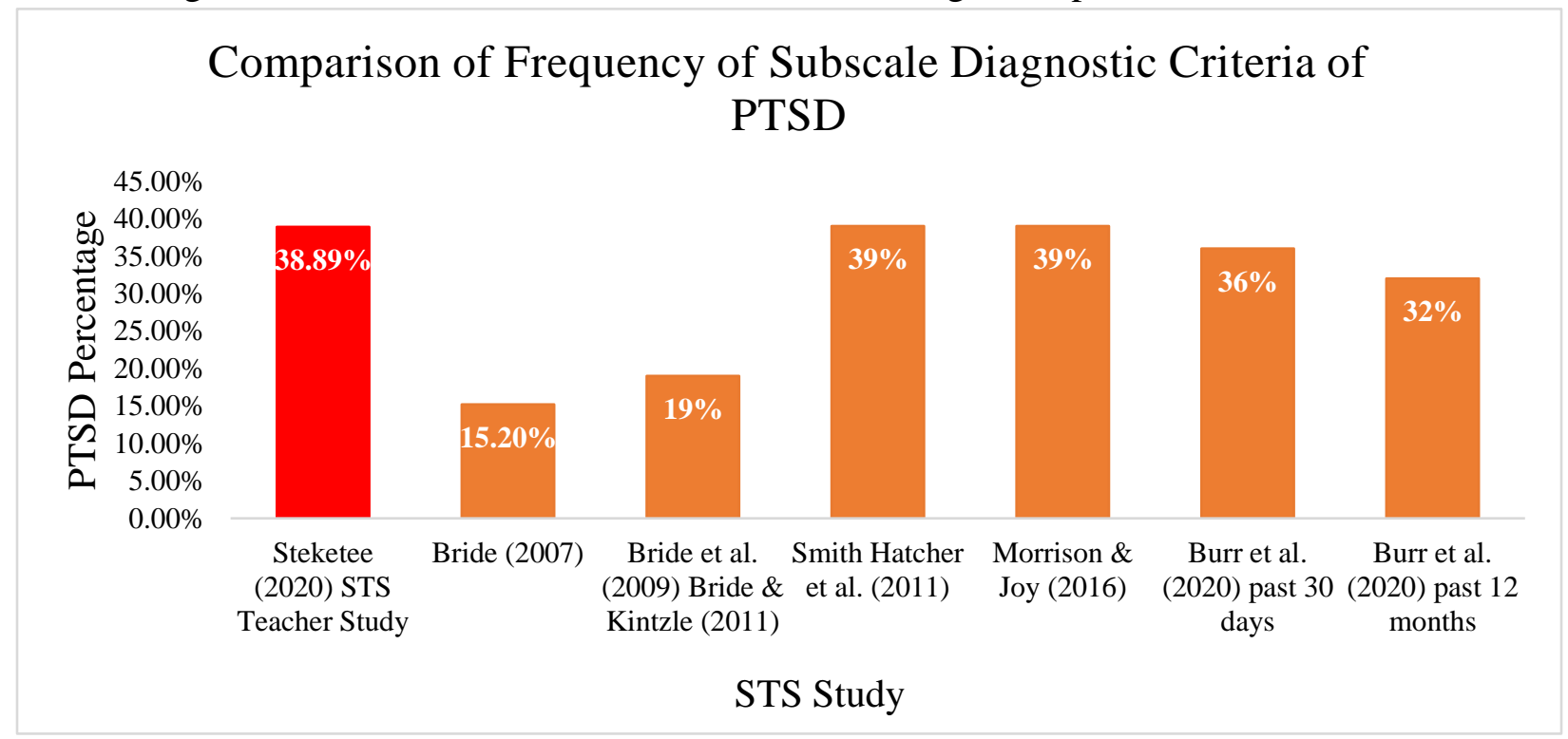

STSS subscale interpretation and comparisons. In addition to the high STSS full scale mean, the teachers in this study also reported high subscale scores. This is reasonable because the whole scale is an aggregate of the subscale scores. Several points support the teachers' high STS subscale scores from this study. With educational professionals, VanBergeijk and Sarmiento (2006) found the three types of symptoms emerged from narratives (plus physical symptoms): intrusive symptoms, cognitive symptoms, and emotional symptoms. Smith Hatcher et al. (2011), working with educators and staff, found that $76.3 \%$ of participants endorsed intrusion symptoms, while $39 \%$ endorsed PTSD symptoms. 39\% of the respondents meeting the PTSD core diagnostic criteria is a high percentage. This is the highest PTSD assessment report (see Figure 
5-4), and it is higher than the global PTSD prevalence, around 10\% (95\% CI: 8.1-11.9\%) for emergency workers.

For the study of teacher STS, using the algorithm interpretation method, teachers in the low opioid zone endorsed all three subscales at $30.67 \%$, in the medium zone at $34.67 \%$, and in the high zone at $51.33 \%$. This last amount, $51.33 \%$, represents 77 respondents who self-reported Intrusion (endorse 1+item at score 3,4,5), Avoidance (endorse 3+ items at score 3,4,5), and Arousal (endorse 2+ items at score 3,4,5). For the entire study, 38.89\% endorsed PTSD symptoms. These findings are highly similar to the findings by Smith Hatcher et al. (2011). The STSS subscales for the teachers are reflected in Tables 47-49, followed by reflections from participants as illustrations of subscale symptoms.

Table 5-5: Results of the STSS Intrusion Score

\begin{tabular}{|c|c|c|c|c|}
\hline STSS & N & M & SD & Interpretation \\
\hline Intrusion Scale & 450 & 12.93 & 4.51 & High/Severe \\
\hline
\end{tabular}

- Florida teacher: "Sometimes I do have their stories bumping around in my head. That's troublesome because out of nowhere, I breathe heavier and feel afraid for my students. At times I've had to tell myself, it's ok, it's ok, you're alright. I don't know if maybe I'm overly sensitive or not."

- Ohio teacher: "It is incredibly challenging to separate my feelings during work and outside of work."

Table 5-6: Results of the STSS Avoidance Score

\begin{tabular}{|c|c|c|c|c|}
\hline STSS & N & M & SD & Interpretation \\
\hline Avoidance Scale & 450 & 16.40 & 6.32 & Weak Moderate \\
\hline
\end{tabular}

- North Carolina teacher: "A feeling of helplessness. Wanting to fix it, and I can't."

- Pennsylvania teacher: "I often feel like there are no tangible ways for me to make their lives better."

- California teacher: "I try to distract myself as much as possible" 
Table 5-7: Results of the STSS Arousal Score

\begin{tabular}{|c|c|c|c|c|}
\hline STSS & N & M & SD & Interpretation \\
\hline Arousal Scale & 450 & 12.73 & 4.77 & Moderate \\
\hline
\end{tabular}

- Texas teacher: "Dealing with their experiences. It's hard to do my work sometimes."

- New Hampshire teacher: "You can't stop thinking about what some of these kids have been through."

- Texas teacher: "I work out to release anger and steam."

The intrusion subscale is endorsed at the highest percentage for participants in the study, followed by arousal, and then avoidance. At the question-level of the STSS, intrusive thoughts (71.8\%) and difficulty sleeping (66.6\%) account for this higher level of intrusion.

Figure 5-5: STSS Subscales of Teachers Using Algorithm Scoring

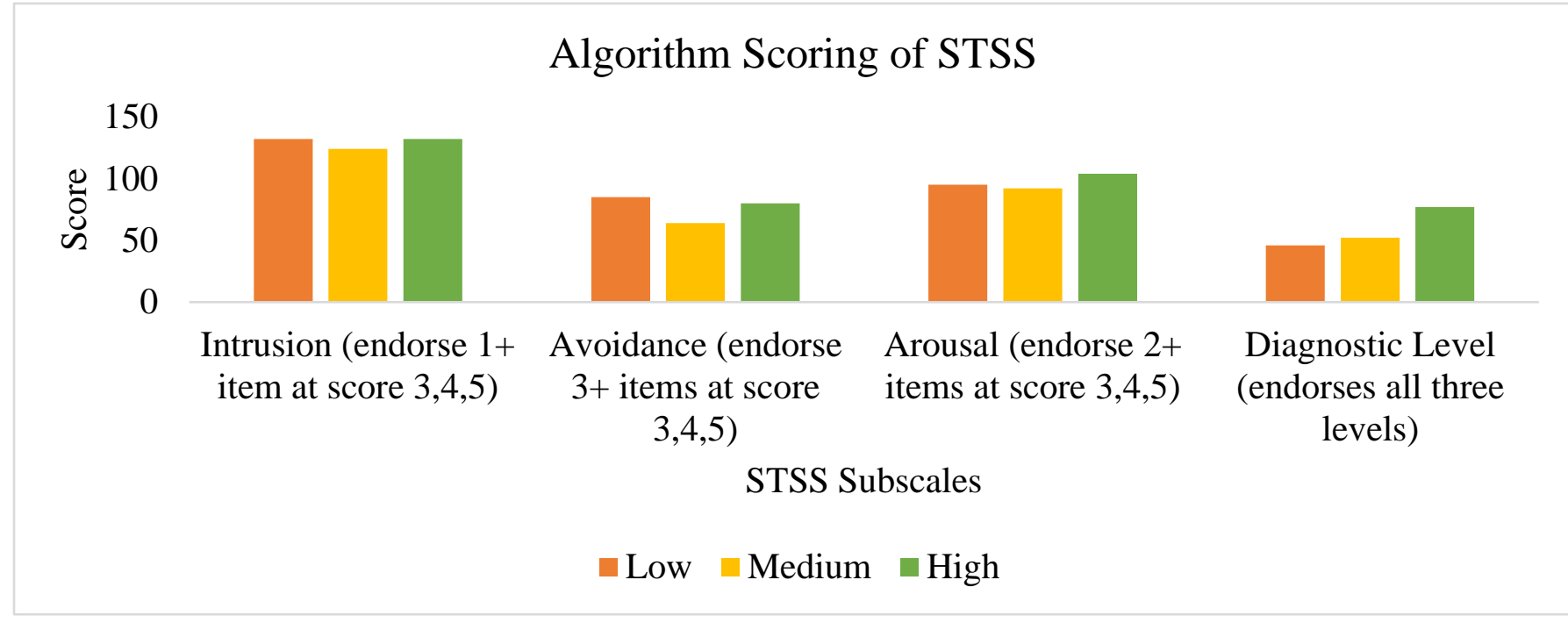

Conclusion. Taken together, the full score and the subscale score are high for the teachers who participated in this study. The results, though higher than other total score STSS findings, appear to be an accurate reflection for this population.

\subsubsection{Relationship of Teacher-Reported STS to Teacher Characteristics}

In addition to the prevalence of teacher-reported STS, the research questions for this study sought to understand the relationship between STS and other variables, like living in 
communities impacted by various levels of opioid mortality, teacher age, gender, race/ethnicity, and relationship status. The remaining two research questions will be explored in this section.

Research Question 2: Is there a relationship between teachers' self-reported levels of secondary traumatic stress when teachers are from states of high-opioid impact, states of medium-opioid impact, and states of low-opioid impact? The second research question relies on correlational measures, which were used in the study to show whether there was a relationship between teachers' STS and their opioid impact area. After the variable of opioid zone was first ranked, and then the STSS scores were ranked, Spearman's rank correlation coefficient (Spearman's Rho) was used to examine whether the STSS scores increased or decreased in correlation with the opioid epidemic zone. On the whole STSS instrument, $\rho=.029$; on the avoidance scale, $\rho=.026$; on the intrusion scale, $\rho=.027$; and on the arousal scale, $\rho=.034$. All of these are positive in direction but negligible. In essence, there is no relationship between the opioid zone and STS.

At first, this finding seems contrary to Denham (2018), who found that the environment (schools of disrepair) had a strong negative impact on STSS scores for teachers. The Spearman's Rho correlation finding of this study did not find a relationship between the environment of the opioid zone (increasing mortality rate, MR, and annual percent of change, APC, of mortality). There may be several reasons for this. First, it is possible that the MR and APC are not the types of indicators that are noted by educators in schools. Second, there may be other indicators of environmental stress that were not considered, such as percent of students on free and reduced lunch program, average housing price in the zip code of schools, other census indicators, state or national rankings, and other indicators of school performance. It is also possible that STS is more 
of a universal construct for teachers, regardless of the community. Finally, although teachers mentioned abuse, neglect, death, and trauma, not one teacher mentioned drugs specifically.

These findings, however, are contrary to the conclusions from Welby (2019), who interviewed 76 elementary school district administrators, teachers, counselors, mental health providers, and consultants using case study methodology in three opioid impacted communities. Welby's study sought to explore the ramifications of the opioid epidemic on elementary schools; because of this purpose, the questions were opioid-related. The educators in Welby's study mentioned STS as an outcome of working in a high-stress school and underscored the need for further training and intervention for STS. Welby reflected that the STS finding was surprising.

Given the overwhelming anecdotal accounts from teachers in communities and schools impacted by the opioid epidemic, I expected a stronger correlation between the opioid epidemic zone and STS. On further reflection, both the teacher STS study and Welby's (2019) study have a commonality: student trauma. When comparing the teachers' perceptions of student Adverse Childhood Experiences (ACEs) to Welby's coded categories, the percentages are similar (see Table 5-8). It is possible that the construct that predicts STS for teachers is not the environment of the opioid zone, per se, but rather the result of the impact of the environment on children: trauma.

Table 5-8: Comparison of PHL-ACEs to Welby (2019) Trauma Categories

\begin{tabular}{|l|c|c|c|c|c|}
\hline \multicolumn{1}{|c|}{ ACEs Types } & $\begin{array}{c}\text { Total } \\
\text { \% }\end{array}$ & $\begin{array}{c}\text { Low } \\
\text { Zone \% }\end{array}$ & $\begin{array}{c}\text { Medium } \\
\text { Zone \% }\end{array}$ & $\begin{array}{c}\text { High } \\
\text { Zone \% }\end{array}$ & $\begin{array}{c}\text { Welby } \\
\text { (2019) }\end{array}$ \\
\hline $\begin{array}{l}\text { Emotional } \\
\text { Abuse }\end{array}$ & $61 \%$ & $58 \%$ & $61 \%$ & $65 \%$ & $\begin{array}{c}76 \% \\
\text { (verbal abuse) }\end{array}$ \\
\hline $\begin{array}{l}\text { Experienced } \\
\text { Death in Family }\end{array}$ & $52 \%$ & $51 \%$ & $57 \%$ & $47 \%$ & $\begin{array}{c}56 \% \\
\text { (death of a parent) }\end{array}$ \\
\hline $\begin{array}{l}\text { Emotional } \\
\text { Neglect }\end{array}$ & $50 \%$ & $55 \%$ & $48 \%$ & $49 \%$ & $\begin{array}{c}93 \% \\
\text { (Neglect) }\end{array}$ \\
\hline
\end{tabular}




\begin{tabular}{|l|c|c|c|c|c|}
\hline $\begin{array}{l}\text { Lived in Foster } \\
\text { Care }\end{array}$ & $48 \%$ & $49 \%$ & $53 \%$ & $42 \%$ & $\begin{array}{c}68 \% \\
\text { (Placed in foster care) }\end{array}$ \\
\hline $\begin{array}{l}\text { Household } \\
\text { Substance Abuse }\end{array}$ & $47 \%$ & $48 \%$ & $46 \%$ & $46 \%$ & $\begin{array}{c}51-59 \% \\
\text { (Observed overdose - } \\
\text { born addicted to opioids) }\end{array}$ \\
\hline
\end{tabular}

Although Spearman's Rho revealed no correlation, there were differences in scores between the zones. The means of the total STSS and the subscales were not significantly related to the opioid zones, but it is possible that the finding that there were more high scorers in the high zone was related to the stress of teaching in a high impact zone. This suggests that the risk of secondary traumatic stress is not equally distributed in the zones but might be clustered more in one zone or another. A different measure of correlation on different variables might determine this in future studies.

Table 5-9: Results of the STSS Scale by Opioid Zone

\begin{tabular}{|l|c|c|c|c|}
\hline \multicolumn{1}{|c|}{ Opioid Zone } & $\boldsymbol{N}$ & $\boldsymbol{M}$ & $\boldsymbol{S D}$ & Interpretation \\
\hline Low & 150 & 42.16 & 14.71 & Strong- Moderate \\
\hline Medium & 150 & 40.23 & 13.44 & Moderate \\
\hline High & 150 & 43.78 & 16.00 & Moderate/High \\
\hline Total (All three zones) & 450 & 42.06 & 14.79 & Strong-Moderate \\
\hline
\end{tabular}

Research Question 3: Is there a relationship between self-reported levels of secondary

traumatic stress and the characteristics of teachers? The last research question is also correlational. Spearman's Rho was used to show whether there was a relationship between teachers' STS and the demographic variables of age, gender, race/ethnicity, and relationship status. Each of these will be discussed as a sub-question.

Research Question 3a: Is there a relationship between self-reported levels of secondary traumatic stress and the age of teachers? After the variable of age was first ranked, and then the STSS scores were ranked, Spearman's rank correlation coefficient (Spearman's Rho) was used 
to examine whether the STSS scores increased or decreased in correlation with age. On the whole STSS instrument, $\rho=-.081$; on the avoidance scale, $\rho=-.061$; on the intrusion scale, $\rho=-$ .077 ; and on the arousal scale, $\rho=-.091$. All of these are negative in direction but negligible. There is no relationship between age and STS. If the Spearman's Rho were a larger number, the negative correlation would imply that as age increases, the STSS score decreases.

Research Question 3b: Is there a relationship between self-reported levels of secondary traumatic stress and gender? After the variable of gender was first ranked, and then the STSS scores were ranked, Spearman's rank correlation coefficient (Spearman's Rho) was used to examine whether the STSS scores increased or decreased in correlation with the gender. On the whole STSS instrument, $\rho=.070$; on the avoidance scale, $\rho=.089$; on the intrusion scale, $\rho=$ .036 ; and on the arousal scale, $\rho=.036$. All of these are positive in direction but negligible. There is no relationship between gender and STS.

When Spearman's Rho was correlated using the aggregated data by opioid zone samples $(n=150$ each) instead of the whole study sample $(\mathrm{N}=450)$, the outputs were slightly different. However, the correlations were still weak or negligent. In the low opioid zone, $\rho=-0.104$, and in the high zone, $\rho=-0.129$. This is compared to the intrusion scale for the total population $(\rho=$ $.036)$.

Research Question 3c: Is there a relationship between self-reported levels of secondary traumatic stress and race/ethnicity? After the variable of race/ethnicity was first ranked, and then the STSS scores were ranked, Spearman's rank correlation coefficient (Spearman's Rho) was used to examine whether the STSS scores increased or decreased in correlation with the race/ethnicity. On the whole STSS instrument, $\rho=-.072$; on the avoidance scale, $\rho=-.072$; on 
the intrusion scale, $\rho=-.114$; and on the arousal scale, $\rho=-.020$. All of these are negative in direction but negligible. There is no relationship between race/ethnicity and STS.

Research Question 3d: Is there a relationship between self-reported levels of secondary traumatic stress and relationship status? After the variable of relationship status was first ranked, and then the STSS scores were ranked, Spearman's rank correlation coefficient (Spearman's Rho) was used to examine whether the STSS scores increased or decreased in correlation with the relationship status. On the whole STSS instrument, $\rho=-.003$; on the avoidance scale, $\rho=.016$; on the intrusion scale, $\rho=-.048$; and on the arousal scale, $\rho=.008$. All of these are positive in direction but negligible. There is no relationship between relationship status and STS. The whole test and the intrusion scale are in the negative direction, and the avoidance and arousal scales are in the positive direction; however, regardless of direction, there is no correlation.

Interpretation of relationship findings. The finding that no demographic variables are associated with teacher STSS scores seems to agree with research on STS in other professionals. Although Hensel et al. (2015), in a meta-analysis of risk factors for therapeutic professionals' STS, identified 17 risk factors which included small significant effect sizes, these did not include demographic variables. Little to no relationship has been found between traumatic stress and age, ethnicity, or income level of trauma therapists (Pearlman \& Mac Ian, 1995). Bride et al. (2004) found no correlations between traumatic stress and social worker age $(r=-.093)$, ethnicity $(r=-$ $.026)$, or income $(r=.095)$. Robinson $(2005)$ found that demographic variables were not able to predict risk for stress in teachers.

Possible alternative consideration. A possible alternative consideration for high STS scores but no correlation to opioid zone or demographic variables lies in the research that does 
show a weak but persistent association between both caseload and previous trauma history. Hensel et al. (2015), in meta-analysis of risk factors for therapeutic professionals' STS, identified 17 risk factors which included small significant effect sizes for trauma caseload volume $(r=.16)$, caseload frequency $(\mathrm{r}=.12)$, caseload ratio $(\mathrm{r}=.19)$, and having a history that included personal trauma ( $\mathrm{r}=.19)$. Bride et al. (2004) also found that the STSS self-report score correlated with the percentage of traumatized clients on a social worker caseload $(M=3.19, \mathrm{SD}=.87, r=.260)$, as well as the time social workers engaged with trauma issues when working with clients $(\mathrm{M}=$ $3.49, \mathrm{SD}=.93, \mathrm{r}=.232)$. Secondary traumatic stress has also been correlated with the extent and intensity of work with traumatized clients (Chrestman, 1999).

The risk factor of previous trauma history would require more research. The anomalous findings by Christian-Brandt et al. (2020), where teachers' report of their intentions to leave the field of education due to stress was not associated with secondary traumatic stress ( $\beta=.03, p=$ $.081)$ and perceptions of trauma-informed care $(\beta=.02, p=.127)$. These findings might reflect that teachers who are either impacted currently by stress or teachers who need trauma intervention are finding benefit in the trauma-informed care model. The benefit of the traumainformed model might also be felt by teachers who have higher depression and anxiety symptoms: Bride et al. (2004) found that the STSS correlated with depression symptoms ( $M=$ $1.74, S D=.79, r=.502)$, and anxiety symptoms $(M=.88, S D=.85, r=.553)$. More work would be needed to understand if these associations have any relationship with teachers who have a prior trauma history.

The risk factor of caseload frequency as a possible explanation for high STS is noted in the literature for helping professionals. Hensel et al. (2015) found that the caseload ratio $(r=.19)$ had the most substantial effect. This means that the "proportion of traumatized clients or 
proportion of time spent working with trauma survivors may matter more than the actual number of individuals or frequency of support” (p. 87). Caseload frequency was subsequently defined as the regular frequency of contact with clients who were traumatized. By noting both the ratio risk and the frequency, it is possible to consider the amount of time an educator might spend with a student who is coping with a traumatizing event, as reflected by the participants of this study.

- Missouri teacher: "I have no training in psychology or trauma. Students want to confide in me, but I can only listen as much as feels appropriate and encourage them to speak with a counselor. I am afraid of saying the wrong thing."

- Colorado teacher: "It gave me depression and anxiety over all the years."

When previous trauma and caseload frequency intersect for teachers, it is possible to consider that STS might be elevated:

- Missouri teacher: "I was abused as a child. I know what these kids are going through."

- Louisiana teacher: "Reminded me of my own trauma."

Conversely, it might be that not having a background or experience with trauma might be its own stressor, as reflected here:

- Ohio teacher: "I do not know how to relate to the students. As someone who has not experienced any trauma personally, I find it hard to communicate with students who have and how to best support them."

Conclusion. Teacher demographics, which include the communities in which they live and their identities with regard to age, race, etc., are not related to the STS self-reported scores obtained by teachers in this study. These results appear to be accurate and are supported by additional research in the field. Two other risk factors, previous history of trauma and caseload frequency, have been postulated as possible future considerations for investigation into teacher STS. 


\subsection{Limitations of the Study}

There are several categories of limitations to this study. These areas will be examined before a discussion of the implications and future research.

\subsubsection{Various Models}

To examine STS in teachers, researchers might benefit from considering various models of trauma to determine their fit. Because current discussions of STS are framed around a medicalized definition of trauma from PTSD diagnostic criteria, it is crucial to explore how the designation of STS carries with it the idea of deficit or impairment. The medicalization of the diagnostic process is detailed and precise, tied to symptoms, and filled with the language of impairment, disturbance, disorder, distortion, negative, detachment, diminished, inability, avoidance, and intrusive. The medical model is looking for evidence of impairment, not resiliency. This pathologized notion of trauma might not be the best fit for teachers working with students in environments of high stress; therefore, additional frameworks of trauma should be explored to determine if they are more useful in educational contexts.

\subsubsection{Teachers with "Disabilities"}

Related to the medical model, more information about teachers with disabilities would inform research about STS in schools. The medical model has three defining features. According to Strauss (2013), these are oriented by definition, location, and treatment. First, defining conditions (illnesses, abnormalities, complaints, disabilities) are pathologized: they are either deficits or exceeding a so-called normal standard. Where deficit is concerned, this excess is on the "lack" end of the normalized curve. Where something like body temperature or amount of pain is involved, the excess is on the "abundance" end of the bell curve. Illness or disability is either a deficit or an excess. Second, this pathology is located within an individual in a particular 
location. By contrast, it is not located in the community or environment. Third, the medical model's goal is that of treatment, driven first by diagnostics. Future research could include more studies about STS for helping professionals in general, and for educational professionals specifically, overtly addressing the medical model.

Additionally, special education highlights the tension surrounding issues of equity and normativity. In education, these tensions coalesce around general education, special education, and inclusive education (Cosier \& Ashby, 2016; Danforth, 2014): special education locates difference and disability within the student and disability studies, with an emphasis on full inclusion, understands difference to be a social construction which is impacted by political and cultural experiences (Goodley, 2013). In other words, special education is rooted in the medical model with a need for evaluation, diagnosis, and remediation of deficits to normalize a student. At the same time, the field of disability studies is more focused on the barriers within the environment and society, which then creates or constructs and reifies the idea of disability (Cosier \& Ashby, 2016). These two divergent paths-special education often leading toward segregation and social construction moving toward full inclusion—exist concurrently, but not without antagonism. Dudley-Marling and Burns (2014) explained that these two perspectives are "each underpinned by sharply different, non-compatible notions of disability" (p. 28). The deficit perspective and medical model dominates in special education, and the social constructivist perspective is reflected in advocacy literature, political discourse, academic discussion, and school reform efforts.

Teachers are aware of these narratives and the social constraints therein. As participants in educational institutions, they move in circles where the evaluation of norms (content norms, intellectual norms, developmental norms) is part of their trade. These discussions of ability and 
normativity are embedded within the operationalization of special education. Future conversations for all teachers, and especially for teachers in special education, might benefit from notions of social construction or a perspective informed by disability studies.

\subsubsection{Limited Number of Studies}

The area of STS is one that has a limited number of studies. Most of these studies focus on people in social work and the medical field. On the one hand, there is much to learn from adopting a transdisciplinary viewpoint. On the other hand, the research focusing on applicability to teachers is very sparse. For teachers, there is useful research that speaks to a portion of the teacher experience: research on teacher depression, research associated with teacher stress on different issues, anecdotal information on teacher stress associated with student trauma, international studies, and limited studies featuring teachers in the United States. With a limited number of studies on teacher secondary stress, comparisons are also somewhat limited.

\subsubsection{Survey Limitations}

There are several limitations to the survey and the survey design. These include the applicability of the STSS survey for additional professionals and the choices made to bound the study. These areas are detailed below.

Survey applicability issues. To assess STS, professionals use several different measures. One of these, the STSS (Bride et al., 2004), is designed for use with therapists, although the authors noted that it could be differentiated for other helping professions. Motta (2015) described that the STSS "lacks empirically derived cut-off scores that are based on standardized measures" (p. 74). Motta (2015) cited the same strengths and weaknesses for the Traumatic Stress Institute Belief Scale (TSI: Pearlman, 1996), the Compassion Satisfaction and Fatigue Test (CSFT: Figley \& Stamm, 1996), and the Compassion Fatigue Scale-Revised (CFS-R: Adams et al., 2006). The 
Secondary Trauma Scale (STS: Motta, Hafeez, Sciancalepore, \& Diaz, 2001) does have cut-off scores and applies to a broader range of adults, but it does not apply to children.

Survey choices. In addition to survey applicability, there were choices made that limited the study concerning decisions about the survey. First, the STSS instrument was chosen over other instruments to study secondary traumatic stress. It is possible, given the paucity of research with teachers, that another instrument would have delivered different findings with more applicability, especially given the critique by Motta (2015) regarding cut-off scores. The scores on the STSS that were derived by percentile and algorithm were useful in that they were comparable to those reported by other researchers.

Regardless of the survey instrument, though, another limitation was the choice of teacher population. By selecting only K-12 teachers, preschool and early education teachers were not included, nor were professors from institutions of higher education. To fully understand teacher STS, it might be useful to have a complete picture of STS in the full education cycle, with teachers from early education and preschool through adult learning. This way, we come closer to understanding if STS is inherent in teaching or, instead, if STS is a construct that is particular to the K-12 setting. Likewise, only public-school teachers were surveyed to the exclusion of private school educators. A comparison could help researchers understand STS with more granularity.

Although great care was taken to define opioid epidemic zones according to current research concerning mortality, there are other ways to consider the impact of stress in communities. In comparing the three opioid zones, there was greater ethnic diversity in the teacher sample in the low opioid zone. The low zone sample was $48 \%$ White, compared to the medium zone (72\%) and high zone (70\%). The low zone also had a higher prevalence of Hispanic teachers, at $24 \%$, compared to $6 \%$ each for the medium and high zones. Schools in the 
low opioid might also have had a different demographic student population, a different poverty indicator, or other differences that might have been important to study. By selecting a zone based on mortality rate, and then not examining other indicators in the communities and schools, the study is limited.

Finally, the study was limited by the participants who did and did not answer the questionnaire. It is representative of those who responded, with the limitations noted in this section. However, the study was limited by the participants who did not answer. It is possible that another cohort of teachers would have had different levels of STS. Because this study only reflects those who responded and not the teachers who did not take the survey, this survey is an accurate reflection only for the 450 teachers who answered.

\subsubsection{Disagreement about Diagnostic Features}

Not all experts agree on the diagnostic features of STS, compassion fatigue, or even PTSD. Because PTSD is the diagnosis upon which the framework of STS is based, it is essential to look at the information limiting the PTSD diagnostic paradigm. For example, Young (1995), eschewing PTSD as a PTSD-based diagnosis at all, calls the categorization "man-made" (p. 141), juxtaposed to the more reality-based and truly felt despair and unhappiness. Young (2004) questioned the entire classification of PTSD in the DSM, based on "connecting its symptoms to an etiology (traumatic experience) and pathogenic mechanism (traumatic memory)" (p. 127). Young encouraged continued diagnostic clarification, especially in the area of traumatic memory typology (p. 142).

Limited notion of trauma. Certain notions of trauma have received more time, funding, and, therefore, focus in the research literature; however, additional trauma constructs exist that were not considered as part of the framework of this study which — for issues pertaining to 
students, teachers, and equity — resulted in limiting the scope and voice of this research. For example, Schepers (2017) frames an STS discussion around insidious trauma, coming from the critical theory of trauma, to include issues like poverty and marginalization. Cates (2013) described insidious trauma as "repetitive demonization of emotionality during development and beyond" (p. 37). The traumatized student is demonized for even having feelings - the psychologically existential equivalent of gaslighting. Similar to insidious trauma, but more focused on one area, is racial trauma, in the framework of cumulative and cultural trauma (Williams, Metzger, Leins, \& DeLapp, 2018). The racial trauma framework posits that it is possible that trauma exposures or events, as currently conceived in the DSM-V, might benefit from a wider lens to include the distress of racism and racially traumatic events. Finally, broader than insidious trauma is the idea of historical trauma, defined by the U.S. Department of Health and Human Services (Trauma: What is Historical Trauma, n.d.) as the following:

Historical trauma is multigenerational trauma experienced by a specific cultural, racial or ethnic group. It is related to major events that oppressed a particular group of people because of their status as oppressed, such as slavery, the Holocaust, forced migration, and the violent colonization of Native Americans. (para.1)

Building on this, Gone et al. (2019) noted that indigenous historical trauma—like racial trauma - "grapples with contextual influences on psychosocial and health phenomena to better appreciate the experiences of historically oppressed and socially marginalized populations" ( $\mathrm{p}$. 32). Compared to other forms of trauma, insidious trauma, racial trauma, and historical trauma may not be as easy to measure, but their importance is difficult to overstate.

This dissertation on teacher STS utilized an expanded version of the ACE assessment to categorize trauma, including racism and poverty. This was important to gain a greater 
understanding of the trauma experienced by children in our communities around the nation. The study, however, is limited in not fully exploring these important additional notions of trauma. Whether we choose to acknowledge these forms of trauma or not, teachers' experiences of secondary traumatic stress are inherently tied to students' experiences of trauma, in all their forms.

\subsection{Implications, Strengths, and Future Research}

Teachers experience secondary stress (Alisic et al., 2012; Robinson, 2005; Rojas-Flores et al., 2015; Schepers, 2017; Smith Hatcher et al., 2011; VanBergeijk \& Sarmiento, 2006). Teachers in high-stress communities also experience secondary stress (Anderson et al., 2019; Caringi et al., 2015; Christian-Brandt et al., 2020; Denham, 2018; Welby, 2019; Wolf-Prusan, 2014). This study aimed to add to the literature regarding teacher STS with implications and recommendations for future research.

\subsubsection{Implications}

This study found an unusually high level of STS for a national sample of public-school K-12 teachers. This STS level does not appear to be related to the fact that teachers are or are not in communities impacted by various opioid mortality rates. The STS level also does not appear to be related to teacher demographics of age, gender, race/ethnicity, or relationship status.

The three subscales of the STSS provide additional information regarding the mechanism of secondary stress in the sample of teachers. Intrusion is the subscale endorsed at the highest percentage among teachers $(86.22 \%)$. The question on the STSS that was the most salient for the score was the following: "I thought about my work with students when I didn't intend to."

This question received a 3, 4, or 5 by $71.8 \%$ of teachers. Teachers, in their responses to questions, echoed this theme: 
- Missouri teacher: "I try to mentally leave work at work and focus on my family when I'm at home."

- New Jersey teacher: "I worry about their future."

The intrusion subscale endorsement is not only high for the study; it is high compared to other studies of STS.

Figure 5-6: Comparison of the Percentage of Teachers in STS Study Who Endorsed Intrusion Symptoms

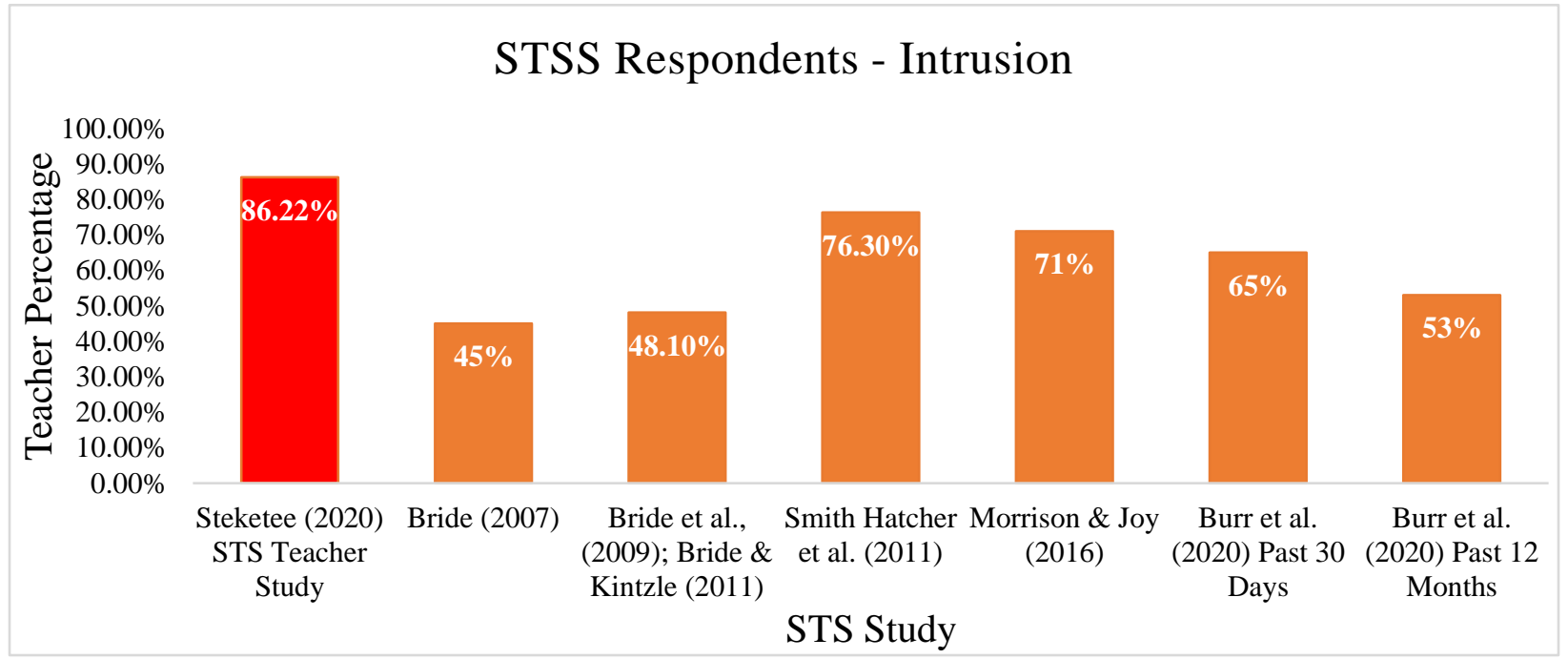

Intrusive thought or worry was counterbalanced, though, by a theme that was not assessed by the Likert-survey instrument. Instead, it was communicated in the open-ended questions: a sense of vocational purpose. The teachers reflected a strong sense of vocational purpose. Although the STSS did not assess for this, it was such a strong theme throughout the open-ended questions that it should be mentioned here. Vocational purpose was expressed by teachers in these ways:

- Texas: "It is a difficult yet rewarding job when I'm able to help someone in need."

- Pennsylvania: "It can be challenging but rewarding."

- New Jersey: "It's rewarding when you have made even a small impact."

- California: "It's hard but rewarding and needed." 
- North Carolina: "It is tough but so worth it."

- Georgia: "It is one of the hardest and best jobs I have ever had. I would not trade my students for the world."

The teachers in the study had high levels of STS, animated by intrusive thoughts. Still, the vocational purpose found in being a teacher may be part of a coping mechanism or a mechanism of resilience. The research of Wolf-Prusan (2014) with teachers in communities of high stress supports the idea of teacher resilience having a mediating effect. The prevalence of the vocational purpose theme cannot be overstated.

Reliving student trauma is another area of difficulty for teachers, as reflected by the high percentage of teachers endorsing this response on the STSS (54.2\%), and the teachers who reflected on this theme in the open-ended questions. It was also underscored by the high percentages that teachers noted for student trauma categories. Saunders and Adams (2014) asserted it would be beneficial for the data to be gathered into a national surveillance system for childhood trauma. Not only would this help social workers and psychologists, but it might support schools and teachers who work with children of trauma. Like Smith Hatcher et al. (2011), whose respondents reported that 95\% of their students experienced trauma, the respondents of this teacher STS study reported that $92 \%$ of their students experienced trauma. Welby (2019) gathered data in three schools and found that respondents at one school reported that $100 \%$ of children had experienced trauma. Similar to the tracking of outbreaks and epidemics, it might be time to consider childhood trauma as an epidemic.

Dealing with student trauma and the impact of student trauma in the classroom and the teachers' lives led teachers to describe their desire for more training:

- Alabama teacher: "There are so many children experiencing these traumas that we need to a better plan to assist students."

- Missouri teacher: "It is difficult. Teachers should get more training in this area." 
- Massachusetts teacher: "Teachers need more training."

- Texas teacher: "We are severely lacking education regarding trauma with our students."

- North Carolina teacher: "Not enough updated training to help them through their difficult moment."

- Arizona teacher: "[...] more training for those of us who work in this field could be very helpful."

- California teacher: "I wish as teachers we got more training on how to properly handle students dealing with trauma."

- Wisconsin teacher: "I need more training!"

The trauma-informed model (Cole et al., 2013) is one that supports both students who have trauma history (Cole et al., 2005) and teachers (Christian-Brandt et al., 2020). Additionally, Caringi et al. (2015) have piloted STAT (Support for Teachers Affected by Trauma, 2020), which is a five-module course, offered free, with information and concrete suggestions for teachers (https://statprogram.org/training). The modules include the following: 1) traumatic stress and teachers, 2) secondary traumatic stress risk factors, 3) assessing for secondary traumatic stress, 4) how STS impacts teachers, and 5) self-care.

\subsubsection{Strengths of the Study}

The study findings are, in themselves, the main strength of the study. The STS level in teachers has not been addressed adequately in research in the United States. This study surveyed a diverse sample of educators in 26 states and Washington, D.C., in three opioid epidemic zones. The sample size $(\mathrm{N}=450)$ is the largest STS study of teachers, to date, and was adequate for the purposes of this research. The completion rate of $100 \%$ was excellent due to the process used in collaboration with Qualtrics. The questionnaire was based on the STSS, which already has a rich history of use in the United States and internationally. It is hoped that other researchers will continue building on the findings in this study and that the additional data collected will continue 
to form a clearer picture of the secondary traumatic stress experienced by K-12 public school teachers in the United States.

\subsubsection{Recommendations for Future Research}

Based on the findings of high STS level but no correlations to opioid mortality index nor teacher demographic identity, it is strongly recommended to ascertain if there is a variable or variables that are related to the STS level. There are several variables to consider. The first is prior trauma history, which proceeds from a robust research precedent. The fact that this STS teacher study added to the literature by repeating previous findings that other demographic variables are not related to STS helps to clear a path for the assessment of new variables. To assess this, researchers could give teachers an ACE test or PHL-ACEs test, along with an STSS, and run correlations to see if there is any relationship between prior trauma and secondary trauma.

It would be prudent to assess whether the STS levels in teachers are significant when the ACEs categories of students are considered. For example, if a teacher endorsed four ACE categories for students in a class, is that teacher more or less likely to have higher STS levels than a teacher who endorsed six categories? Relatedly, it would be important to understand the STS levels of teachers who endorse specific ACE categories for students. Teachers who selfreport a classroom with students who have trauma stemming from physical abuse might be at more risk for a higher STS score. Finally, in that same vein, if the ACE categories are found to be significant for teacher STS, it would be necessary to rerun correlations to see if any teacher demographic variables are associated with this STS-ACE pairing.

The number of environmental and socioeconomic variables associated with schooling supports the idea that additional research could be undertaken to ascertain which, if any, 
community variables might be impacting teachers. For example, do teachers from high poverty schools have higher STS? Do teachers in schools with greater ethnic diversity have lower STS? These were issues that were not explored in this dissertation, but they are important questions. The demographics of teachers in each of the three zones had some differences, so it might be worthwhile to understand these differences and how they impact STS.

Additionally, training and intervention programs, like STAT, are excellent candidates for test-retest studies concerning STS. Because teachers stated so clearly in the open-ended questions that they want training, it might be useful to give an STSS, run a training seminar or program, and then administer the STSS again at a later date. Similarly, many different types of interventions could be selected for this type of assessment. It might be possible to tailor interventions based on the three subscales, beginning with intrusion because the intrusion subscale seems to be the most troubling to teachers. In the qualitative data, "talking to others" and "the need to talk to others" were two robust themes for teacher coping. These themes support future directions for both training and interventions.

The qualitative data from 450 teachers in 26 states and Washington, D.C. seem like important research strands to follow, even in descriptive and correlational research. While statistics can offer a picture of prevalence and correlation, the voice of the teacher adds an essential and not-to-be-overlooked stream of data. It is possible to imagine a dialog between the narrative data and the quantitative data (Garcia, López, \& Vélez, 2017). It seems shortsighted to overlook these critical reflections from teachers in the field. For example, this type of data could be quantitatively coded for subscale themes to ascertain the number of times that teachers refer to symptoms of intrusion, avoidance, and arousal. 
Finally, a national teacher STS study seems necessary. The high level of STS in this study does not appear to be an anomaly. It might be supportive for teachers now and in the future to survey teachers through their unions nationally to gain a more comprehensive understanding of STS nationally.

\subsection{Conclusion}

The topic of secondary traumatic stress (STS) in teachers has been explored by considering three questions: who are the teachers who have been affected by STS (population); how pervasively is STS occurring for teachers (prevalence); and in what ways does STS impact the lives of teachers (impact). These are not merely questions for the classroom, where teachers are often considered as a means to a research end, studied to improve the academic outcomes of students, or parsed to push new policy and procedure. These are human rights questions, questions of transformation. Teachers are helping traumatized children in communities of stress. Teachers, though, can pay the price for this sustenance. Stamm (1999) reflected on the importance of communities, "My experiences have shown me how communities, when well cared for, sustain their members during these times of failed self-sufficiency" (p. xvii). During times of crisis like the opioid epidemic, COVID-19 pandemic, or mass civil protest for social change, our teachers provide that sense of care in school settings, in ways that sustain children and families.

To honor those who are sustaining so many, I want to end with the voices of teachers, as they reflect on what it means to be sustainers, even in the midst of secondary traumatic stress:

- California teacher: "Students who have experienced significant adversity and trauma need people in their lives who are ready and willing to step up for them and find ways to support them individually-this is what I endeavor to do for my students daily." 
This sentiment summed up what many educators attempted to reflect in their answers. Yet, this work takes a toll on teachers.

- It is incredibly challenging to separate my feelings during work and outside of work.

- It's difficult because I do not want to do or say the wrong thing.

- I often feel like there are no tangible ways for me to make their lives better.

- [There is] the sadness I feel for the students experiencing trauma and adversity.

- It triggers my own childhood trauma.

- It hurts very much.

- Sometimes I find myself internalizing my students' problems.

- I have to be careful not to get too depressed about the situations.

- It is challenging.

- painful

- be strong

These are the voices animating the $M=42.06(S D=14.79)$ full score, and $M=42.16(S D=$ 14.71), low opioid zone, and $M=40.23(S D=13.44)$ medium opioid zone, and $M=43.78(S D$ $=16.00$ ) high zone. Future research is not just a necessity; it is an ethical imperative. 


\section{References}

Abram, K. M., Teplin, L. A., Charles, D. R., Longworth, S. L., McClelland, G. M., \& Dulcan, M. K. (2004). Posttraumatic stress disorder and trauma in youth in juvenile detention. Archives of General Psychiatry, 61(4), 403-410. doi:10.1001/archpsyc.61.4.403

Achinstein, B., Ogawa, R. T., Sexton, D., \& Freitas, C. (2010). Retaining teachers of color: A pressing problem and a potential strategy for "hard-to-staff" schools. Review of Educational Research, 80(1), 71-107. doi:10.3102/0034654309355994

Adams, R. E., Boscarino, J., \& Figley, C. R. (2006). Compassion fatigue and psychological distress among social workers: A validation study. American Journal of Orthopsychiatry, 76(1), 103-108. doi:10.1037/0002-9432.76.1.103

Advisory Council on the Misuse of Drugs. (2003). Hidden harm: Responding to the needs of children of problem drug users. Retrieved from https://assets.publishing.service.gov.uk/government/uploads/system/uploads/attachment data/file/120620/hidden-harm-full.pdf

Akinsulure-Smith, A. M., Espinosa, A., Chu, T., \& Hallock, R. (2018). Secondary traumatic stress and burnout among refugee resettlement workers: The role of coping and emotional intelligence. Journal of Traumatic Stress, 31(2), 202-212. doi:10.1002/jts.22279

Akoglu, H. (2018). User's guide to correlation coefficients. Turkish Journal of Emergency Medicine, 18(3), 91-93. doi:10.1016/j.tjem.2018.08.001

Albaek, A. U., Kinn, L. G., \& Milde, A. M. (2017). Walking children through a minefield: How professionals experience exploring adverse childhood experiences. Qualitative Health Research, 28(2), 231-244. doi:10.1177/1049732317734828 
Alisic, E. (2012). Teachers' perspectives on providing support to children after trauma: A qualitative study. School Psychology Quarterly, 27(1), 51-59. doi:10.1037/a0028590

Alisic, E., Bus, M., Dulack, W., Pennings, L., \& Splinter, J. (2012). Teachers' experiences supporting children after traumatic exposure. Journal of Traumatic Stress, 25(1), 98-101. doi: $10.1002 /$ jts. 20709

Altshuler, S. J., \& Cleverly-Thomas, A. (2011). What do we know about drug endangered children when they are first placed into care? Child Welfare, 90(3), 45-68. Retrieved from https://pubmed.ncbi.nlm.nih.gov/22403900/

Alvarez, D. (2010). "I had to teach hard": Traumatic conditions and teachers in post-Katrina classrooms. The High School Journal, 94(1), 28-39. Retrieved from https://www.jstor.org/stable/40981100

American Psychiatric Association. (1994). Diagnostic and statistical manual of mental disorders (4th ed.). Washington, DC: Author.

American Psychiatric Association. (2000). Diagnostic and statistical manual of mental disorders (4th ed., text rev.). Washington, DC: Author.

American Psychiatric Association (2013). Diagnostic and statistical manual of mental disorders, (5th ed.). Washington, DC: Author.

American Psychological Association. (2010). Publication manual of the American Psychological Association (6th ed.). Washington, DC: Author.

American Psychological Association. (2017). Clinical practice guideline for treatment of posttraumatic stress disorder (PTSD) in adults. Retrieved from https://www.apa.org/ptsd-guideline/ptsd.pdf

Anda, R. F., Butchart, A., Felitti, V. J., \& Brown, D. W. (2010). Building a framework for global 
surveillance of the public health implications of adverse childhood experiences.

American Journal of Preventive Medicine, 39(1), 93-98.

doi:10.1016/j.amepre.2010.03.015

Anderson, S., Troilo, J., \& Tack, F. (2019). Crisis in the classroom: West Virginia teachers and the opioid epidemic. West Virginia University. Retrieved from https://cehs.wvu.edu/crisis-in-the-classroom?wvu

Armour, C., Carragher, N., Elhai, J.D. (2013). Assessing the fit of the dysphoric arousal model across two nationally representative epidemiological surveys: The Australian NSMHWB and the United States NESARC. Journal of Anxiety Disorders, 27(1), 109-115. doi:10.1016/j.janxdis.2012.10.006

Armour, C., Tsai, J., Durrham, T., Charak, R., Biehn, T., Elhai, J., \& Pietrak, R. (2015). Dimensional structure of DSM-5 posttraumatic stress symptoms: Support for a hybrid Anhedonia and Externalizing Behaviors model. Journal of Psychiatric Research, 61, 106-113. doi:10.1016/j.jpsychires.2014.10.012

Atallah, D. G., Koslouski, J. B., Perkins, K. N., Marsico, C., \& Porche, M. V. (2019). An evalutation of Trauma and Learning Policy Initiative's (TLPI) Inquiry-Based Process: Year three. Retrieved from Trauma Sensitive Schools website: https://traumasensitiveschools.org/wp-content/uploads/2020/05/BU_Report_Final.pdf

Bachem, R., Mitreuter, S., Levin, Y., Stein, J. Y., Xiao, Z., \& Solomon, Z. (2018). Longitudinal development of primary and secondary posttraumatic growth in aging veterans and their wives: Domain-specific trajectories. Journal of Traumatic Stress, 31(5), 730-741. doi: $10.1002 /$ jts.22331

Badger, K., Royse, D., \& Craig, C. (2008). Hospital social workers and indirect trauma 
exposure: An exploratory study of contributing factors. Health \& Social Work, 33(1), 63-71. doi:10.1093/hsw/33.1.63

Baird, K., \& Kracen, A. C. (2006). Vicarious traumatization and secondary traumatic stress: A research synthesis. Counselling Psychology Quarterly, 19(2), 181-188. doi:10.1080/09515070600811899

Barnett, J., Diaz, M., Merz, S., Redfield, C., Wawro, T., \& West, B. (2018). The teacher leadership competencies. Retrieved from National Education Association website: http://www.nea.org/assets/docs/NEA_TLCF_20180824.pdf

Baum, N. L., Rotter, B., Reidler, E., \& Brom, D. (2009). Building resilience in schools in the wake of Hurricane Katrina. Journal of Child and Adolescent Trauma, 2(1), 62-70. doi:10.1080/ 19361520802694323

Beckmann, S. (2015). Secondary traumatic stress and posttraumatic growth: Risk and protective factors among American Red Cross disaster responders and disaster mental health workers. (Doctoral dissertation). Retrieved from ProQuest Dissertations and Theses database. (UMI No. 3728149)

Beckwith, A. M., \& Burke, S. A. (2015). Identification of early developmental deficits in infants with prenatal heroin, methadone, and other opioid exposure. Clinical Pediatrics, 54(4), 328-335. doi:10.1177/0009922814549545

Bellingrath, S., \& Kudielka, B. (2017). Biological pathways to stress-related disease vulnerability in educators. In T. McIntyre, S. McIntyre, \& D. Francis (Eds.), Educator stress: An occupational health perspective (pp. 77-100). Cham, Switzerland: Springer International Publishing.

Ben-Porat, A., \& Itzhaky, H. (2011). The contribution of training and supervision to perceived 
role competence, secondary traumatization, and burnout among domestic violence therapists. The Clinical Supervisor, 30(1), 95-108. doi:10.1080/07325223.2011.566089

Benson, J., \& Magraith, K. (2005). Compassion fatigue and burnout: The role of Balint groups. Australian Family Physician, 34(6), 497-498. Retrieved from https://hekyll.services.adelaide.edu.au/dspace/bitstream/2440/17227/1/hdl_17227.pdf

Benuto, L. T., Yang, Y., Ahrendt, A., \& Cummings, C. (2018). The Secondary Traumatic Stress Scale: Confirmatory factor analyses with a national sample of victim advocates. Advance online publication, Mar 11. Journal of Interpersonal Violence, 1-20. Retrieved from https://journals.sagepub.com/doi/10.1177/0886260518759657

Bercier, M. L., \& Maynard, B. R. (2015). Interventions for secondary traumatic stress with mental health workers: A systematic review. Research on Social Work Practice, 25, 8189. doi:10.1177/1049731513517142

Berger, R., Abu-Raiya, H., \& Benatov, J. (2016). Reducing primary and secondary traumatic stress symptoms among educators by training them to deliver a resiliency program (ERASE-Stress) following the Christchurch earthquake in New Zealand. American Journal of Orthopsychiatry, 86(2), 236-251. doi:10.1037/ort0000153

Berger, W., Coutinho, E. S. F., Figueira, I., Marques-Portella, C., Luz, M. P., Neylan, T. C., ... Mendlowicz, M. V. (2011). Rescuers at risk: A systematic review and meta-regression analysis of the worldwide current prevalence and correlates of PTSD in rescue workers. Social Psychiatry and Psychiatric Epidemiology, 47(6), 1001-1011. doi:10.1007/s00127011-0408-2

Bernstein, D. P., Stein, J. A., Newcomb, M. D., Walker, E., Pogge, D., Ahluyalia, T., \& Zule, W. (2013). Development and validation of a brief screening version of the Childhood 
Trauma Questionnaire. Child Abuse \& Neglect, 27(2), 169-90. doi:10.1016/s01452134(02)00541-0

Bethell, C. D., Carle, A., Hudziak, J., Gombojav, N., Powers, K., Wade, R., \& Braveman, P. (2017). Methods to assess adverse childhood experiences of children and families: Toward approaches to promote child well-being in policy and practice. Academic Pediatrics, 17(7), S51-S69. doi:10.1016/j.acap.2017.04.161

Bills, L. J. (1999). Trauma-based psychiatry for primary care. In B. H. Stamm (Ed.), Secondary traumatic stress: Self-care issues for clinicians, researchers, \& educators (2nd ed., pp. 121-148). Lutherville, MD: Sidran Press.

Bisits, P. (2014). How to choose a sample size (for the statistically challenged). Tools4dev.com. Retrieved from http://www.tools4dev.org/resources/how-to-choose-a-sample-size/

Blad, E. (2019, January 17). Opioid crisis: Online course teaches educators how to recognize, respond to addiction. Education Week. Retrieved from http://blogs.edweek.org/edweek/rulesforengagement/2019/01/opioid_crisis_online_cours e_teaches_educators_how to_recognize_respond_to_addiction.html

Blodgett, C., \& Lanigan, J. D. (2018). The association between adverse childhood experience (ACE) and school success in elementary school children. School Psychology Quarterly, 33(1), 137-146. doi:10.1037/spq0000256

Bober, T., \& Regehr, C. (2006). Strategies for reducing secondary or vicarious trauma: Do they work? Brief Treatment and Crisis Intervention, 6(1), 1-9. doi:10.1093/brief-treatment/mhj001

Bonett, D., \& Wright, T. (2015). Cronbach's alpha reliability: Interval estimation, hypothesis 
testing, and sample size planning. Journal of Organizational Behavior, 36(1), 3-15. doi:10.1002/job.1960

Borntrager, C., Caringi, J. C., van den Pol, R., Crosby, L., O'Connell, K., Trautman, A., \& McDonald, M. (2012). Secondary traumatic stress in school personnel. Advances in School Mental Health Promotion, 5(1), 38-50. doi:10.1080/1754730x.2012.664862

Boscarino, J. A., Figley, C. R., \& Adams, R. E. (2004). Compassion fatigue following September 11 terrorist attacks: A study of secondary trauma among New York City social workers. International Journal of Emergency Mental Health, 6, 57-66. Retrieved from https://www.omicsonline.org/international-journal-of-emergency-mental-health-andhuman-resilience.php

Brady, J. L., Guy, J. D., Poelstra, P. L., \& Brokaw, B. F. (1999). Vicarious traumatization, spirituality, and the treatment of sexual abuse survivors: A national survey of women psychotherapists. Professional Psychology: Research and Practice, 30(4), 386-393. doi:10.1037//0735-7028.30.4.386

Branson, D. C. (2019). Vicarious trauma, themes in research, and terminology: A review of literature. Traumatology, 25(1), 2-10. doi:10.1037/trm0000161

Braun, V., \& Clarke, V. (2006). Using thematic analysis in psychology. Qualitative Research in Psychology, 3(2), 77-101. doi:10.1191/1478088706qp063oa

Bride, B. E. (2007). Prevalence of secondary traumatic stress among social workers. Social Work, 52(1), 63-70. doi:10.1093/sw/52.1.63

Bride, B. E., \& Figley, C. R. (2009). Secondary trauma and military veteran caregivers. Smith College Studies in Social Work, 79(3-4), 314-329. doi:10.1080/00377310903130357

Bride, B. E., \& Kintzle, S. (2011). Secondary traumatic stress, job satisfaction, and occupational 
commitment in substance abuse counselors. Traumatology, 17(1), 22-28.

doi:10.1177/1534765610395617

Bride, B. E., Radey, M., \& Figley, C. R. (2007). Measuring compassion fatigue. Clinical Social Work Journal, 35(3), 155-163. doi:10.1007/s10615-007-0091-7

Bride, B. E., Robinson, M. M., Yegidis, B., \& Figley, C. R. (2004). Development and validation of the Secondary Traumatic Stress Scale. Research on Social Work Practice, 14(1), 2735. doi: $10.1177 / 1049731503254106$

Bride, B. E., Smith Hatcher, S., \& Humble, M. N. (2009). Trauma training, trauma practices, and secondary traumatic stress among substance abuse counselors. Traumatology, 15(2), 96105. doi:10.1177/1534765609336362

Browne, T., Evangeli, M., \& Greenberg, N. (2012). Trauma-related guilt and posttraumatic stress among journalists. Journal of Traumatic Stress, 25(2), 207-210. doi:10.1002/jts.21678

Brundage, S., Fifield, A., \& Partridge, L. (2019). The ripple effect: National and state estimates of the U.S. opioid epidemic's impact on children chartbook. Retrieved from United Hospital Fund website https://uhfnyc.org/media/filer_public/6e/80/6e80760f-d579-46a3998d-1aa816ab06f6/uhf_ripple_effect_national_and_state_estimates_chartbook.pdf

Brundage, S. C., \& Levine, C. (2019). The ripple effect: The impact of the opioid epidemic on children and families. Retrieved from United Hospital Fund website: https://uhfnyc.org/media/filer_public/17/2c/172ca968-43aa-45f9-a29050018e85a9d8/uhf-opioids-20190315.pdf

Burr, K. L., O’Brien, P., Brown, J. M., Penfil, S. H., \& Hertzog, J. H. (2020). Occupationalinduced secondary traumatic stress and posttraumatic stress disorder in respiratory therapists. Respiratory Care, 65(7), 1019-1023. doi:10.4187/respcare.06840 
Callaghan, T., Crimmins, J., \& Schweitzer, R. D. (2011). Children of substance-using mothers: Child health engagement and child protection outcomes. Journal of Paediatrics and Child Health, 47(4), 223-227 doi:10.1111/j.1440-1754.2010.01930.x

Cancio, E. J., Larsen, R., Mathur, S. R., Estes, M. B., Johns, B., \& Chang, M. (2018). Special education teacher stress: Coping strategies. Education and Treatment of Children, 41(4), 457-481. doi:10.1353/etc.2018.0025

Cannon, Y., Davis, G., His, A., \& Bochte, A. (2016). Adverse childhood experiences in the New Mexico juvenile justice population, Feb 2016. Georgetown Law Faculty Publications and Other Works, 2191. Retrieved from https://scholarship.law.georgetown.edu/facpub/2191

Caringi, J. C., Stanick, C., Trautman, A., Crosby, L., Devlin, M., \& Adams, S. (2015). Secondary traumatic stress in public school teachers: Contributing and mitigating factors. Advances in School Mental Health Promotion, 8(4), 244-256.

doi:10.1080/1754730x.2015.1080123

Carver-Thomas, D., \& Darling-Hammond, L. (2017). Teacher turnover: Why it matters and what we can do about it. Learning Policy Institute. Retrieved from https://learningpolicyinstitute.org/product/teacher-turnover-report

Cates, L. B. (2013). Insidious emotional trauma: The body remembers ... ${ }^{1}$. International Journal of Psychoanalytic Self Psychology, 9(1), 35-53. doi:10.1080/15551024.2014.857751

Catherall, D. R. (1999). Coping with secondary traumatic stress: The importance of the therapist's professional peer group. In B. H. Stamm (Ed.), Secondary traumatic stress: Self-care issues for clinicians, researchers, \& educators (2nd ed., pp. 80-94). Lutherville, MD: Sidran Press.

Center for Substance Abuse Treatment, Substance Abuse and Mental Health Services 
Administration. (2009). Appendix E: DSM-IV-TR criteria for posttraumatic stress disorder of Substance abuse treatment: Addressing the specific needs of women (TIP No. 51). Retrieved from https://www.ncbi.nlm.nih.gov/books/NBK83241/

Centers for Disease Control and Prevention (CDC). (2004). Prevalence of overweight and obesity among adults with diagnosed diabetes - United States, 1984-1994 and 19992002. Morbidity and Mortality Weekly Report, 53(45), 1066-1068. Washington, DC: Government Printing Office.

Centers for Disease Control and Prevention (CDC). (2009). Behavioral Risk Factor Surveillance System Survey Data. Atlanta, GA: U.S. Department of Health and Human Services, Centers for Disease Control and Prevention. Retrieved from https://www.cdc.gov/brfss/about/archived.htm

Centers for Disease Control and Prevention (CDC). (2010). Behavioral Risk Factor Surveillance System Survey Data. Atlanta, GA: U.S. Department of Health and Human Services, Centers for Disease Control and Prevention. Retrieved from https://www.cdc.gov/brfss/about/archived.htm

Centers for Disease Control and Prevention (CDC). (2011). Behavioral Risk Factor Surveillance System Survey Data. Atlanta, GA: U.S. Department of Health and Human Services, Centers for Disease Control and Prevention. Retrieved from https://www.cdc.gov/brfss/about/archived.htm

Centers for Disease Control and Prevention (CDC). (2012). Behavioral Risk Factor Surveillance System Survey Data. Atlanta, GA: U.S. Department of Health and Human Services, Centers for Disease Control and Prevention. Retrieved from https://www.cdc.gov/brfss/about/archived.htm 
Centers for Disease Control and Prevention. (CDC). (2012a). Principles of Epidemiology in Public Health Practice: An Introduction to Applied Epidemiology and Biostatistics (3rd ed.). Lesson 1: Introduction to epidemiology, Section 11: Epidemic disease occurrence. Retrieved from https://www.cdc.gov/csels/dsepd/ss1978/Lesson1/Section11.html\#_ref51 Centers for Disease Control and Prevention. (CDC). (2012b). Principles of Epidemiology in Public Health Practice: An Introduction to Applied Epidemiology and Biostatistics (3rd ed.). Lesson 3: Measures of risk, Section 2: Morbidity frequency measures. Retrieved from https://www.cdc.gov/csels/dsepd/ss1978/lesson3/section2.html

Centers for Disease Control and Prevention (CDC). (2020a). About the CDC-Kaiser ACE study: Data and statistics. Atlanta, GA: U.S. Department of Health and Human Services, Centers for Disease Control and Prevention. Retrieved from https://www.cdc.gov/violenceprevention/acestudy/about.html

Centers for Disease Control and Prevention (CDC). (2020b). Drug overdose mortality by state. Atlanta, GA: U.S. Department of Health and Human Services, Centers for Disease Control and Prevention. Retrieved from https://www.cdc.gov/nchs/pressroom/sosmap/drug_poisoning_mortality/drug_poisoning. htm

Chafouleas, S. M., Koriakin, T. A., Roundfield, K. D., \& Overstreet, S. (2018). Addressing childhood trauma in school settings: A framework for evidence-based practice. School Mental Health, 11(1), 40-53. doi:10.1007/s12310-018-9256-5

Chan, D. (2009). So why ask me? Are self-report data really that bad? In C. E. Lance \& R. J. Vandenberg (Eds.), Statistical and methodological myths and urban legends: Doctrine, verity and fable in the organizational and social sciences (pp. 309-336). New York: 
Psychology Press.

Chapman, J. K. (2004). A rural-based study of developmental outcomes in young children prenatally exposed to cocaine. Rural Special Education Quarterly, 23(3), 23-29. doi:10.1177/875687050402300304

Chasnoff, I. J., Telford, E., Wells, A. M., \& King, L. (2015). Mental health disorders among children within child welfare who have prenatal substance exposure: Rural vs. urban populations. Child Welfare, 94(4), 53-70. Retrieved from https://pubmed.ncbi.nlm.nih.gov/26827476/

Cherniss, C. (1980). Staff burnout. Beverly Hills, CA: Sage.

Chrestman, K.R. (1999). Secondary exposure to trauma and self-reported distress among therapists. In B. H. Stamm (Ed.), Secondary traumatic stress: Self-care issues for clinicians, researchers, \& educators (2nd ed., pp. 29-36). Lutherville, MD: Sidran Press.

Christian-Brandt, A. S., Santacrose, D. E., \& Barnett, M. L. (2020). In the trauma-informed care trenches: Teacher compassion satisfaction, secondary traumatic stress, burnout, and intent to leave education within underserved elementary schools, Mar 6. Child Abuse \& Neglect, 104437. doi:10.1016/j.chiabu.2020.104437

Christie, C. Baker, C., Cooper, R., Kennedy, P. J., Madras, B., \& Bondi, P. (2017). The president's commission on combating drug addiction and the opioid crisis. Washington, DC: Government Printing Office.

Cieslak, R., Shoji, K., Douglas, A., Melville, E., Luszczynska, A., \& Benight, C. C. (2014). A meta-analysis of the relationship between job burnout and secondary traumatic stress among workers with indirect exposure to trauma. Psychological Services, 11(1), 75-86. doi:10.1037/a0033798 
Coker, A. L., McKeown, R. E., Sanderson, M., Davis, K. E., Valois, R. F., \& Huebner, E. S. (2000). Severe dating violence and quality of life among South Carolina high school students. American Journal of Preventive Medicine, 19(4), 220-227. doi:10.1016/s07493797(00)00227-0

Cole, S. F., Eisner, A., Gregory, M., \& Ristuccia, J. (2013). Helping traumatized children learn: Creating and advocating for trauma-sensitive schools (Vol. 2). Boston, MA: Massachusetts Advocates for Children.

Cole, S. F., O’Brien, J. G., Gadd, M. G., Ristuccia, J., Wallace, D. L., \& Gregory, M. (2005). Helping traumatized children learn: Supportive school environments for children traumatized by family violence (Vol. 1). Boston, MA: Massachusetts Advocates for Children.

Collie, R. J., Perry, N. E., \& Martin, A. J. (2017). School context and educational system factors impacting educator stress. In T. McIntyre, S. McIntyre, \& D. Francis (Eds.), Educator stress: An occupational health perspective (pp. 3-22). Cham, Switzerland: Springer International Publishing.

Collier, L. (2018). Young victims of the opioid crisis. American Psychological Association. Retrieved from https://www.apa.org/about/awards/search.aspx

Collins, C. (2018). The opioid crisis. Teaching Tolerance. Retrieved from https://www.tolerance.org/magazine/summer-2018/the-opioid-crisis

Comer, J. P. (2005). Child and adolescent development: The critical missing focus in school reform. Phi Delta Kappan, 86(10), 757-763. doi:10.1177/003172170508601008 
Copeland, W. E., Keeler, G., Angold, A., \& Costello, E. J. (2007). Traumatic events and posttraumatic stress in childhood. Archives of General Psychiatry, 64(5), 577-584. doi:10.1001/archpsyc.64.5.577

Cosier, M., \& Ashby, C. (2016). Disability studies and the "work" of educators. In M. Cosier \& C. Ashby (Eds.), Enacting change from within: Disability studies meets teaching and teacher education (pp. 1-19). New York, NY: Peter Lang.

Costello, E. J., Erkanli, A., Fairbank, J. A., \& Angold, A. (2002). The prevalence of potentially traumatic events in childhood and adolescence. Journal of Traumatic Stress, 15(2), 99112. doi:10.1023/a:1014851823163

Couper, M. P. (2000). Web surveys: A review of issues and approaches. Public Opinion Quarterly, 64(4), 464-494. doi:10.1086/318641

Courage, M. M., \& Williams, D. M. (1986). An approach to the study of burnout in professional care providers in human service organizations. Journal of Social Service Research, 10(1), 7-22. doi:10.1300/j079v10n01_03

Creswell, J., \& Guetterman, T. (2019). Educational research: Planning, conducting, and evaluating quantitative and qualitative research (6th Edition). New York, NY: Pearson.

Cronbach, L. J. (1951). Coefficient alpha and the internal structure of tests. Psychometrika, 16(3), 297-334. doi:10.1007/BF02310555

Cronholm, P. F., Forke, C. M., Wade, R., Bair-Merritt, M. H., Davis, M., Harkins-Schwarz, M., ... Fein, J. A. (2015). Adverse childhood experiences: Expanding the concept of adversity. American Journal of Preventive Medicine,49(3), 354-361. doi:10.1016/j.amepre.2015.02.001

Cummings, C., Singer, J., Hisaka, R., \& Benuto, L. T. (2018). Compassion satisfaction to 
combat work-related burnout, vicarious trauma, and secondary traumatic stress. Journal of Interpersonal Violence. Advanced online publication. doi:10.1177/0886260518799502

Danforth, S. (2014). Becoming a great inclusive educator. New York, NY: Peter Lang.

Davies, H., Gilbert, R., Johnson, K., Petersen, I., Nazareth, I., O’Donnell, ... GonzalezIzquierdo, A. (2015). Neonatal drug withdrawal syndrome: Cross-country comparison using hospital administrative data in England, the USA, Western Australia and Ontario, Canada. Archives of Disease in Childhood: Fetal and Neonatal Edition, 101(1), 26-30. doi:10.1136/archdischild-2015-308948

Denham, F. S. (2018). School building blight and teacher secondary traumatic stress: A quantitative study (Doctoral dissertation). Retrieved from PQDT Open. (No. 10974259)

Department of Justice, National Heroin Task Force. (2015). National Heroin Task Force final report and recommendations. Retrieved from https://www.justice.gov/file/822231/download

Derefinko, K. J., Salgado García, F. I., Talley, K. M., Bursac, Z., Johnson, K. C., Murphy, J. G., ... Sumrok, D. D. (2019). Adverse childhood experiences predict opioid relapse during treatment among rural adults. Addictive Behaviors, 96, 171-174. doi:10.1016/j.addbeh.2019.05.008

Devilly, G. J., Wright, R., \& Varker, T. (2009). Vicarious trauma, secondary traumatic stress or simply burnout? Effect of trauma therapy on mental health professionals. Australian \& New Zealand Journal of Psychiatry, 43(4), 373-385. doi:10.1080/00048670902721079

DeVoe, E. R., Klein, T. P., Bannon, W., \& Miranda-Julian, C. (2011). Young children in the aftermath of the World Trade Center attacks. Psychological Trauma: Theory, Research, Practice, and Policy, 3(1), 1-7. doi:10.1037/a0020567

Dirks, A. (2018). The opioid epidemic: Impact on children and families. Journal of Psychiatry 
and Psychiatric Disorders, 2(1), 9-11. doi:10.26502/jppd.2572-519x0035

Draugalis, J. R., Coons, S. J., \& Plaza, C. M. (2008). Best practices for survey research reports: A synopsis for authors and reviewers. American Journal of Pharmaceutical Education, 72(1), 11. doi:10.5688/aj720111

Dudley-Marling, C., \& Burns, M. B. (2013). Two perspectives on inclusion in the United States. Global Education Review, 1(1), 14-31. Retrieved from http://ger.mercy.edu/index.php/ger

Edelman, M. W. (2017, October). Children and the opioid crisis. Children's Defense Fund. Retrieved from https://www.childrensdefense.org/child-watchcolumns/health/2017/children-and-the-opioid-crisis/

Elhai, J. D., Biehn, T. L., Armour, C., Klopper, J. J., Frueh, B. C., \& Palmieri, P.A. (2011). Evidence for a unique PTSD construct represented by PTSD's D1-D3 symptoms. Journal of Anxiety Disorders, 25, 340-345. doi:10.1016/j.janxdis.2010.10.007

Engberg, J., \& Morral, A. R. (2006). Reducing substance use improves adolescents' school attendance. Addiction, 101(12), 1741-1751. doi:10.1111/j.1360-0443.2006.01544.x

Fantuzzo, J. W., Perlman, S. M., \& Dobbins, E. K. (2011). Types and timing of child maltreatment and early school success: A population-based investigation. Children and Youth Services Review, 33, 1404-1411. doi:10.1016/j.childyouth.2011.04.010

Feder, K. (2018). Children in the United States opioid epidemic (Doctoral dissertation). Retrieved from ProQuest Dissertations and Theses database. (UMI No. 27606938)

Feder, K. A., Letourneau, E. J., \& Brook, J. (2019). Children in the opioid epidemic: Addressing the next generation's public health crisis. Pediatrics, 143(1), 1-3. doi:10.1542/peds.20181656 
Felitti, V. J., Anda, R. F., Nordenberg, D., Williamson, D. F., Spitz, A. M., Edwards, V., ... Marks, J. S. (1998). Relationship of childhood abuse and household dysfunction to many of the leading causes of death in adults: The adverse childhood experiences (ACE) study. American Journal of Preventive Medicine, 14(4), 245-258. doi:10.1016/s07493797(98)00017-8

Figley, C. R. (1995). Compassion fatigue as secondary traumatic stress disorder: An overview. In C. R. Figley (Ed.), Compassion fatigue: Coping with secondary traumatic stress disorder in those who treat the traumatized (pp. 1-20). New York, NY: Routledge.

Figley, C. R. (1996). Compassion Fatigue Self Test. In B. H. Stamm (Ed.), Measurement of stress, trauma, \& adaptation (pp. 129-130). Lutherville, MD: Sidran Press.

Figley, C. R. (1999). Compassion fatigue: Toward a new understanding of the costs of caring. In B. H. Stamm (Ed.), Secondary traumatic stress: Self-care issues for clinicians, researchers, \& educators (2nd ed., pp. 3-28). Lutherville, MD: Sidran Press.

Figley, C. R. (Ed.). (2002). Treating compassion fatigue. New York, NY: BrunnerRoutledge.

Figley, C. R., \& Stamm, B. H. (1996). Psychometric review of Compassion Fatigue Self Test. In B. H. Stamm (Ed.), Measurement of stress, trauma, and adaptation (pp. 127-128). Lutherville, MD: Sidran Press.

Fill, M.-M. A., Miller, A. M., Wilkinson, R. H., Warren, M. D., Dunn, J. R., Schaffner, W., \& Jones, T. F. (2018). Educational disabilities among children born with neonatal abstinence syndrome. Pediatrics, 142(3), 252-258. doi:10.1542/peds.2018-0562

Finkelhor, D., Hammer, H., \& Sedlak, A. J. (2002). Non-family abducted children: National estimates and characteristics (NCJ 196467). Retrieved from U.S. Department of Justice, 
Office of Juvenile Justice and Delinquency Prevention website:

https://ojjdp.ojp.gov/library/publications/nonfamily-abducted-children-nationalestimates-and-characteristics

Finkelhor, D., Ormrod, R., Turner, H., \& Hamby, S. L. (2005). The victimization of children and youth: A comprehensive, national survey. Child Maltreatment, 10(1), 5-25. doi:10.1177/1077559504271287

Finkelhor, D., Turner, H., Ormrod, R., \& Hamby, S. L. (2009). Violence, abuse, and crime exposure in a national sample of children and youth. Pediatrics, 124, 1411-1423. doi.org/10.1542/peds.2009-0467

Flannelly, K. J., Roberts, S. B., \& Weaver, A. J. (2005). Correlates of compassion fatigue and burnout in chaplains and other clergy who responded to the September 11th attacks in New York City. The Journal of Pastoral Care and Counseling, 59, 213-224. doi:10.1177/154230500505900304

Florence, C. S., Zhou, C., Luo, F., \& Xu, L. (2016). The economic burden of prescription opioid overdose, abuse, and dependence in the United States, 2013. Medical Care, 54(10), 901906. doi:10.1097/mlr.0000000000000625

Franklin, C. G. S., Kim, J. S., Ryan, T. N., Kelly, M. S., \& Montgomery, K. L. (2012). Teacher involvement in school mental health interventions: A systematic review. Children and Youth Services Review, 34(5), 973-982. doi:10.1016/j.childyouth.2012.01.027

Fuchs, D., \& Fuchs, L. S. (2006). Introduction to Response to Intervention: What, why, and how valid is it? Reading Research Quarterly, 41(1), 93-99. doi:10.1598/RRQ.41.1.4

Garcia, N. M., López, N., \& Vélez, V. N. (2017). QuantCrit: Rectifying quantitative methods 
through critical race theory. Race Ethnicity and Education, 21(2), 149-157.

doi:10.1080/13613324.2017.1377675

Garza, G., \& Landrum, B. (2013). Mending fences: Defining the domains of quantitative and qualitative research. Qualitative Psychology, 2(2), 199-209. doi:10.1037/e588692013001

Gentry, J. E., Baranowsky, A. B., \& Dunning, K. (2002). The Accelerated Recovery Program (ARP) for compassion fatigue. In C. R. Figley (Ed.), Treating compassion fatigue (pp. 123-138). New York, NY: Brunner-Routledge.

George, D., \& Mallery, P. (2003). SPSS for Windows step by step: A simple guide and reference, 11.0 update (4th ed.). Boston, MA: Allyn \& Bacon.

Ghertner, R., Baldwin, M., Crouse, G., Radel, L., \& Waters, A. (2018). The relationship between substance use indicators and child welfare caseloads. Retrieved from U.S. Department of Health and Services, Office of the Assistant Secretary for Planning and Evaluation website: https://aspe.hhs.gov/system/files/pdf/258831/SubstanceUseCWCaseloads.pdf

Ghertner, R., Waters, A., Radel, L., \& Crouse, G. (2018). The role of substance use in child welfare caseloads. Children and Youth Services Review, 90, 83-93. doi:10.1016/j.childyouth.2018.05.015

Gil, S., \& Weinberg, M. (2015). Secondary trauma among social workers treating trauma clients: The role of coping strategies and internal resources. International Social Work, 58(4), 551-561. doi:10.1177/0020872814564705

Glen, S. (2014, December 8). Cronbach's alpha: Simple definition, use and interpretation. Statistics How To. Retrieved from https://www.statisticshowto.com/cronbachs-alpha-spss/ Goldring, R., Taie, S., \& Riddles, M. (2014). Teacher attrition and mobility: Results from the 
2012-23 Teacher Follow-up Survey (NCES 2014-077). Retrieved from U.S. Department of Education, National Center for Education Statistics website: https://nces.ed.gov/pubs2014/2014077.pdf

Gone, J. P., Hartmann, W. E., Pomerville, A., Wendt, D. C., Klem, S. H., \& Burrage, R. L. (2019). The impact of historical trauma on health outcomes for indigenous populations in the USA and Canada: A systematic review. American Psychologist, 74(1), 20-35. doi:10.1037/amp0000338

Gonzalez, A., Monzon, N., Solis, D., Jaycox, L., \& Langley, A. K. (2016). Trauma exposure in elementary school children: Description of screening procedures, prevalence of exposure, and posttraumatic stress symptoms. School Mental Health, 8(1), 77-88 doi:10.1007/s1231 0-015-9167-7.

Goodley, D. (2013) Dis/entangling critical disability studies, Disability \& Society, 28(5), 631644. doi:10.1080/09687599.2012.717884

Goodman, R. D., Miller, M. D., \& West-Olatunji, C. A. (2012). Traumatic stress, socioeconomic status, and academic achievement among primary school students. Psychological Trauma: Theory, Research, Practice, and Policy, 4(3), 252-259. doi:10.1037/a0024912

Granek, L., Nakash, O., Cohen, M., Ben-David, M., \& Ariad, S. (2017). Oncologists' communication about end of life: The relationship among secondary traumatic stress, compassion satisfaction, and approach and avoidance communication. PsychoOncology, 26(11), 1980-1986. doi:10.1002/pon.4289

Gray, K., Capote, N., \& Valiente, A. (2016, March 9). New Hampshire middle schoolers are learning how to use an antidote to stop a heroin overdose. ABC News. 
https://abcnews.go.com/Health/hampshire-middle-schoolers-learning-antidote-stopheroin-overdose/story?id=37499734

Guerra, C., \& Saiz, J. L. (2007). Examen psicométrico de la Escala de Estrés Traumático Secundario: Un estudio en profesionales chilenos [Psychometric examination of the Secondary Traumatic Stress Scale: A study on Chilean professionals.]. Psicología Conductual, 15(3), 441-456. Retrieved from https://dialnet.unirioja.es/revista/2034/A/2007

Gutermann, J., Schreiber, F., Matulis, S., Schwartzkopff, L., Deppe, J., \& Steil, R. (2016). Psychological treatments for symptoms of posttraumatic stress disorder in children, adolescents, and young adults: A meta-analysis. Clinical Child and Family Psychology Review, 19(2), 77-93. doi:10.1007/s10567-016-0202-5

Harland, N., \& Holey, E. (2011). Including open-ended questions in quantitative questionnaires - theory and practice. International Journal of Therapy and Rehabilitation, 18(9), 482-486. doi:10.12968/ijtr.2011.18.9.482

Hayes, C. (2006). Stress relief for teachers: The 'coping triangle. 'New York, NY: Routledge. Health Federation of Philadelphia and Philadelphia ACE Research and Data Committee. (2012). Philadelphia ACE survey. Philadelphia, PN: Philadelphia ACE Project. Retrieved from https://www.philadelphiaaces.org/resources

Hefling, K., \& Stratford, M. (2018, June 18). Opioid epidemic overwhelms schools. Politico: Morning Education. Retrieved from https://www.politico.com/newsletters/morningeducation/2018/06/18/opioid-epidemic-overwhelms-schools-257387

Herman, K. C., Hickmon-Rosa, J., \& Reinke, W. M. (2017). Empirically Derived Profiles of Teacher Stress, Burnout, Self-Efficacy, and Coping and Associated Student Outcomes. 
Journal of Positive Behavior Interventions, 20(2), 90-100.

doi:10.1177/1098300717732066

Hensel, J. M., Ruiz, C., Finney, C., \& Dewa, C. S. (2015). Meta-analysis of risk factors for secondary traumatic stress in therapeutic work with trauma victims. Journal of Traumatic Stress, 28(2), 83-91. doi:10.1002/jts.21998

Herranz, G. S, Vílchez, M. A. L., Ledo, J. D., \& Sierra, A. M. (2014). Children born to heroinaddicted mothers: What's the outcome 25 years later? Journal of Addiction Research \& Therapy, 5(2), 1-6. doi:10.4172/2155-6105.1000180

Hser, Y. I., Mooney, L. J., Saxon, A. J., Miotto, K., Bell, D. S., Zhu, Y., ... Huang, D. (2017). High mortality among patients with opioid use disorder in a large healthcare system. Journal of Addiction Medicine, 11(4), 315-319. doi:10.1097/adm.0000000000000312

Hudak, M. L., Tan, R.C., Committee on Drugs, \& Committee on Fetus and Newborn. (2012). Neonatal drug withdrawal. Pediatrics, 129(2), 540-560. doi:10.1542/peds.2011-3212

Hussar, B., Zhang, J., Hein, S., Wang, K., Roberts, A., Cui, J., ... Dilig, R. (2020). The condition of education 2020 (NCES 2020-144). Retrieved from U.S. Department of Education, National Center for Education Statistics website: https://nces.ed.gov/pubsearch/pubsinfo.asp?pubid=2020144

Hydon, S., Wong, M., Langley, A. K., Stein, B. D., \& Kataoka, S. H. (2015). Preventing secondary traumatic stress in educators. Child and Adolescent Psychiatric Clinics of North America, 24(2), 319-333. doi:10.1016/j.chc.2014.11.003

Ireland, C. A., \& Huxley, S. (2018). Psychological trauma in professionals working with traumatised children. The Journal of Forensic Practice, 20(3), 141-151. doi:10.1108/jfp10-2017-0045 
Isaac, S., \& Michael, W. B. (1995). Handbook in research and evaluation: A collection of principles, methods, and strategies useful in the planning, design, and evaluation of studies in education and the behavioral sciences (3rd ed.). San Diego, CA: EdITS Publishers.

Jacobs, I., Charmillot, M., Martin Soelch, C., \& Horsch, A. (2019). Validity, reliability, and factor structure of the Secondary Traumatic Stress Scale-French Version. Frontiers in Psychiatry, 10, 1-11. doi:10.3389/fpsyt.2019.00191

Jaycox, L. H., Kataoka, S. H., Stein, B. D., Langley, A. K., \& Wong, M. (2012) Cognitive behavioral intervention for trauma in schools. Journal of Applied School Psychology, 28(3), 239-255, doi:10.1080/15377903.2012.695766

Jenkins, S. R., \& Baird, S. (2002). Secondary traumatic stress and vicarious trauma: A validational study. Journal of Traumatic Stress, 15(5), 423-432. doi:10.1023/A:1020193526843

Jones, C. M., Logan, J., Gladden, R. M., \& Bohm, M. K. (2015). Vital signs: Demographic and substance use trends among heroin users - United States, 2001-2013. Morbidity and Mortality Weekly Report, 64(26), 719-725. Retrieved from https://www.ncbi.nlm.nih.gov/pmc/articles/PMC4584844/pdf/719-725.pdf

Kassam-Adams, N. (1999). The risks of treating sexual trauma: Stress and secondary trauma in psychotherapists. In B. H. Stamm (Ed.), Secondary traumatic stress: Self-care issues for clinicians, researchers, and educators (2nd ed., pp. 37-50). Lutherville, MD: Sidran.

Kelley-Quon, L. I. (2018). Surveys: Merging qualitative and quantitative research methods. Seminars in Pediatric Surgery, 27(6), 361-366. doi:10.1053/j.sempedsurg.2018.10.007 
Kiang, M. V., Basu, S., Chen, J., \& Alexander, M. J. (2019). Assessment of changes in the geographical distribution of opioid-related mortality across the United States by opioid type, 1999-2016. JAMA Network Open, 2(2), 1-10.

doi:10.1001/jamanetworkopen.2019.0040

King, D. W., Leskin, G. A., King, L. A., \& Weathers, F. W. (1998). Confirmatory factor analysis of the clinician-administered PTSD scale. Psychological Assessment, 10(2), 90-96. doi:10.1037/1040-3590.10.2.90.

Klein, R. (2018, November 03). Teachers are serving as first responders to the opioid crisis. Huffington Post. Retrieved from https://m.huffpost.com/us/entry/us_5bd9c9c4e4b01abe6a1a4206/amp

Kolaitis, G. (2017). Trauma and post-traumatic stress disorder in children and adolescents. European Journal of Psychotraumatology, 8(4), 1-2. doi:10.1080/20008198.2017.1351198

Krupnik, V. (2019). Trauma or adversity? Traumatology, 25(4), 256-261. doi:10.1037/trm0000169

Kyriacou, C. (2001). Teacher stress: Directions for future research. Educational Review, 53(1), 27-35. doi:10.1080/00131910124115

La Greca, A. M., Boyd, B. A., Jaycox, L. H., Kassam-Adams, N., Mannarino, A. P., Silverman, W. K., ... Wong, M. (2008). Children and trauma--Update for mental health professionals: APA presidential task force on posttraumatic stress disorder and trauma in children \& adolescents. Retrieved from American Psychological Association website: https://www.apa.org/pi/families/resources/update.pdf

Lander, L., Howsare, J., \& Byrne, M. (2013). The impact of substance use disorders on 
families and children: From theory to practice. Social Work in Public Health, 28(3-4), 194-205. doi:10.1080/19371918.2013.759005

Landers, J. (2018, Sept. 26). Helping teachers manage the weight of trauma. Usable Knowledge. Retrieved from https://www.gse.harvard.edu/news/uk/18/09/helping-teachers-manageweight-trauma

Lane, K. L., Oakes, W., \& Menzies, H. (2010). Systematic screenings to prevent the development of learning and behavior problems: Considerations for practitioners, researchers, and policy makers. Journal of Disability Policy Studies, 21(3), 160-172. doi: $10.1177 / 1044207310379123$

Lansford, J. E., Dodge, K. A., Pettit, G. S., Bates, J. E., Crozier, J., \& Kaplow, J. (2002). A 12year prospective study of the long-term effects of early child physical maltreatment on psychological, behavioral, and academic problems in adolescence. Archives of Pediatrics and Adolescent Medicine, 156, 824-830. doi:10.1001/archpedi.156.8.824

Larson, M. G. (2006). Descriptive statistics and graphical displays. Circulation, 114(1), 76-81. doi:10.1161/circulationha.105.584474

Lee, J. H., Lee, D., Kim, J., Jeon, K., \& Sim, M. (2017). Duty-related trauma exposure and posttraumatic stress symptoms in professional firefighters. Journal of Traumatic Stress, 30(2), 133-141. doi:10.1002/jts.22180

Leeb, R. T., Lewis, T., \& Zolotor, A. J. (2011). A review of physical and mental health consequences of child abuse and neglect and implications for practice. American Journal of Lifestyle Medicine, 5, 454- 468. doi:10.1177/1559827611410266

Lessler, J., Azman, A. S., McKay, H. S., \& Moore, S. M. (2017). What is a hotspot anyway? The American Journal of Tropical Medicine and Hygiene, 96(6), 1270-1273. 
doi:10.4269/ajtmh.16-0427

Levine, C. (2018, January 2). The statistics don't capture the opioid epidemic's impact on children. STAT. https://www.statnews.com/2018/01/02/opioid-epidemic-impact-children/

Levine, P. A., \& Kline, M. (2007). Trauma through a child's eyes: Awakening the ordinary miracle of healing. Berkeley, CA: North Atlantic Books.

Lipari, R. N., \& Van Horn, S. L. (2017). Children living with parents who have a substance use disorder (CBHSQ Report No. 3223). Retrieved from Substance Abuse and Mental Health Services Administration website https://www.samhsa.gov/data/sites/default/files/report_3223/ShortReport-3223.pdf

Litvinov, A. (2019, January 30). Teaching the children of the opioid crisis. NEAToday. Retrieved from http://neatoday.org/2019/01/14/teaching-the-children-of-the-opioid-crisis/

Liu, P., Wang, L., Cao, C., Wang, R., Zhang, J., Zhang, B., ... Elhai, J. D. (2014). The underlying dimensions of DSM-5 posttraumatic stress disorder symptoms in an epidemiological sample of Chinese earthquake survivors. Journal of Anxiety Disorders, 28, 345-351. doi:10.1016/j.janxdis.2014.03.008

Lodico, M. G., Spaulding, D. T., \& Voegtle, K. H. (2010). Methods in educational research: From theory to practice. San Francisco, CA: Jossey-Bass.

Lopez, C. (2019, April). Analyzing data. [PowerPoint slides]. Chapman University. Ludick, M., \& Figley, C. R. (2017). Toward a mechanism for secondary trauma induction and reduction: Reimagining a theory of secondary traumatic stress. Traumatology, 23(1), 112-123. doi: $10.1037 /$ trm0000096 
Macrae, J., \& Hyde, P. (2015, July 27). HHS launches multipronged effort to combat opioid abuse. [Web log post]. Retrieved from http://www.hhs.gov/blog/2015/07/27/hhs$\underline{\text { launches-multipronged-effort-combat-opioid-abuse.html }}$

Madras, B. K. (2018). The president's commission on combating drug addiction and the opioid crisis: Origins and recommendations. Clinical Pharmacology \& Therapeutics, 103(6), 943-945. doi:10.1002/cpth.1050

Marcinowicz, L., Chlabicz, S., \& Grębowski, R. (2007). Open-ended questions in surveys of patients' satisfaction with family doctors. Journal of Health Services Research \& Policy, 12(2), 86-89. doi:10.1258/135581907780279639

Markwell, A. L., \& Wainer, Z. (2009). The health and wellbeing of junior doctors: Insights from a national survey. Medical Journal of Australia, 191(8), 441-444. doi:10.5694/j.13265377.2009.tb02880.x

Maslach, C. (2003). Job burnout. Current Directions in Psychological Science, 12(5), 189-192. doi:10.1111/1467-8721.01258

Maslach, C., \& Jackson, S. E. (1981). MBI: Maslach Burnout Inventory. Palo Alto, CA: Consulting Psychologists Press.

Mather, M., Jarosz, B., \& Slowey, M. (2019). A growing number of U.S. children live in counties affected by the drug epidemic. Population Reference Bureau. Retrieved from https://www.prb.org/a-growing-number-of-u-s-children-live-in-counties-affected-by-the$\underline{\text { drug-epidemic/ }}$

McCann, I. L., \& Pearlman, L. A. (1990). Vicarious traumatization: A framework for understanding the psychological effects of working with victims. Journal of Traumatic Stress, 3, 131-149. doi.org/10.1002/jts.2490030110 
McCammon, S. L. (1999). Painful pedagogy: Teaching about trauma in academic and training settings. In B. H. Stamm (Ed.), Secondary traumatic stress: Self-care issues for clinicians, researchers, \& educators (2nd ed., pp. 105-120). Lutherville, MD: Sidran Press.

McCarthy, C., Lineback, S., Fitchett, P., Lambert, R., Eyal, M., \& Boyle, L. (2017). Biological pathways to stress-related disease vulnerability in educators. In T. McIntyre, S. McIntyre, \& D. Francis (Eds.), Educator stress: An occupational health perspective (pp. 77-100). Cham, Switzerland: Springer International Publishing.

McDonald, J. H. (2014). Handbook of biological statistics (3rd ed.). Baltimore, MD: Sparky House Publishing.

McDonald, S. D., \& Calhoun, P. S. (2010). The diagnostic accuracy of the PTSD Checklist: A critical review. Clinical Psychology Review, 30(8), 976-987. doi:10.1016/j.cpr.2010.06.012

McIntyre, T., McIntyre, S., \& Francis, D. (Eds.). (2017). Educator stress: An occupational health perspective. Cham, Switzerland: Springer International Publishing.

McLennan, J., Evans, L., Cowlishaw, S., Pamment, L., \& Wright, L. (2016). Secondary traumatic stress in postdisaster field research interviewers. Journal of Traumatic Stress, 29(1), 101-105. doi:10.1002/jts.22072

Mirick, R. G., \& Steenrod, S. A. (2016). Opioid use disorder, attachment, and parenting: Key concerns for practitioners. Child and Adolescent Social Work Journal, 33(6), 547-557. doi:10.1007/s10560-016-0449-1

Mitchell, N. S., Catenacci, V. A., Wyatt, H. R., \& Hill, J. O. (2011). Obesity: Overview of an epidemic. Psychiatric Clinics of North America, 34(4), 717-732. 
doi:10.1016/j.psc.2011.08.005

Molnar, B. E., Sprang, G., Killian, K. D., Gottfried, R., Emery, V., \& Bride, B. E. (2017). Advancing science and practice for vicarious traumatization/secondary traumatic stress: A research agenda. Traumatology, 23(2), 129-142. doi:10.1037/trm0000122

Montgomery, C., \& Rupp, A. (2005). A meta-analysis for exploring the diverse causes and effects of stress in teachers. Canadian Journal of Education, 28(3), 458-486. doi:10.2307/4126479

Mordeno, I. G., Go, G. P., \& Yangson-Serondo, A. (2017). Examining the dimensional structure models of secondary traumatic stress based on DSM-5 symptoms. Asian Journal of Psychiatry, 25, 154-160. doi:10.1016/j.ajp.2016.10.024

Morrison, L. E., \& Joy, J. P. (2016). Secondary traumatic stress in the emergency department. Journal of Advanced Nursing, 72(11), 2894-2906. doi:10.1111/jan.13030

Motta, R. W., Hafeez, S., Sciancalepore, R., \& Diaz, A. B. (2001). Discriminant validation of the secondary trauma scale. Journal of Psychotherapy in Independent Practice, 24, 17-24. doi:10.1300/J288v02n04_02

Motta, R. W. (2012). Secondary trauma in children and school personnel. Journal of Applied School Psychology, 28(3), 256-269. doi:10.1080/15377903.2012.695767

Motta, R. W. (2015). Trauma, PTSD, and secondary trauma in children and adolescents. In R. Flanagan, K. Allen, \& E. Levine (Eds.), Cognitive and behavioral interventions in the schools: Integrating theory (pp. 67-84). New York, NY: Springer.

Murphy, J. F., \& Tobin, K. J. (2011). Homelessness comes to school. Phi Delta Kappan, 93(3), 32-37. doi:10.1177/003172171109300308

Myers, T. W., \& Cornille, T. A. (2002). The trauma of working with traumatized children. In E. 
Figley (Ed.), Treating compassion fatigue (pp. 39-55). New York: Bruner/Mazel.

Nadiv, S. (2019, March 18). For many struggling in the opioid crisis, school \& early childcare programs provide safe spaces: Q\&A with WestEd's Sarah Nadiv. WestEd Insights. Retrieved from https://www.wested.org/wested-insights/school-and-early-childcareprograms-provide-safe-spaces-qa-with-westeds-sarah-nadiv/

NAIS Surveys FAQ. (2019). NAIS \& surveys of student engagement. Indiana University Bloomington. Retrieved from http://ceep.indiana.edu/nais/faq/index.html

National Child Traumatic Stress Network (NCTSN). (2006). The effects of trauma on schools and learning. Retrieved from https://www.nctsn.org/trauma-informed-care/creatingtrauma-informed-systems/schools

National Child Traumatic Stress Network Schools Committee. (2008). Child trauma toolkit for educators. Retrieved from https://www.nctsn.org/trauma-informed-care/creating-traumainformed-systems/schools

National Child Traumatic Stress Network, Secondary Traumatic Stress Committee. (2011). Secondary traumatic stress: A fact sheet for child-serving professionals [Fact sheet]. Retrieved from https://www.nctsn.org/sites/default/files/resources/fact$\underline{\text { sheet/secondary_traumatic_stress_child_serving_professionals.pdf }}$

National Institute on Drug Abuse. (2018). Opioids and the overdose epidemic. Retrieved from $\underline{\text { https://teens.drugabuse.gov/teachers/lessonplans/opioids-and-overdose-epidemic }}$

National Institute of Mental Health. (2019, May). Post-traumatic stress disorder. Retrieved from https://www.nimh.nih.gov/health/topics/post-traumatic-stress-disorder-ptsd/index.shtml

Neonatal abstinence syndrome. (2019). In A.D.A.M. medical encyclopedia.

Retrieved from https://medlineplus.gov/ency/article/007313.htm 
Newton, D. (2018). The opioid crisis: A reference handbook. Santa Barbara, CA: ABC-CLIO, LLC.

Nimmo, A., \& Huggard, P. (2013). A systematic review of the measurement of compassion fatigue, vicarious trauma, and secondary traumatic stress in physicians. Australasian Journal of Disaster and Trauma Studies, 1, 37-44. Retrieved from http://trauma.massey.ac.nz/

Normile, B., Hanlon, C., \& Eichner, H. (2018). State strategies to meet the needs of young children and families affected by the opioid crisis. Retrieved from National Academy for State Health Policy website: https://www.nashp.org/wpcontent/uploads/2018/09/Children-and-Opioid-Epidemic-1.pdf

Oei, J. L., Melhuish, E., Uebel, H., Azzam, N., Breen, C., Burns, L., ... Wright, I. M. (2017). Neonatal abstinence syndrome and high school performance. Pediatrics, 139(2), 1-10. doi:10.1542/peds.2016-2651

Ohio Department of Education. (2020, January 15). Opioid abuse prevention. Retrieved from http://education.ohio.gov/Topics/Learning-in-Ohio/Health-Education/Opioid-Abuse$\underline{\text { Prevention }}$

33 Ohio Rev. Code $\S 3313.60$ (2014), available at https://law.justia.com/codes/ohio/2014/title-33/chapter-3313/section-3313.60-2/

Olsen, Y., \& Sharfstein, J. M. (2019). The opioid epidemic: What everyone needs to know. New York, NY: Oxford University Press.

Operation Prevention. (2017). Classroom resources. Retrieved from https://www.operationprevention.com/classroom

Overdose Prevention Act. 18A N. J. Rev. Stat. § 40-12.23-12.28 (2013 \& Supp. 2018). 
Pang, V., Madueño, M., Atlas, M., Stratton, T., Oliger, J., \& Page, C. (2008). Addressing student trauma in the wake of the California wildfires. Social Education, 72(1), 18-23. Retrieved from https://www.socialstudies.org/publications/socialeducation

Patrick, S. W., Davis, M. M., Lehmann C. U., \& Cooper, W. O. (2015). Increasing incidence and geographic distribution of neonatal abstinence syndrome: United States 2009 to 2012. Journal of Perinatology, 35, 650-655. doi:10.1038/jp.2015.36

Patrick, S. W., Schiff, D. M., \& Committee of Substance Use and Prevention. (2017). A public health response to opioid use in pregnancy. Pediatrics, 139(3), 1-7. doi:10.1542/peds.2016-4070

Pearlman, L. A., \& Mac Ian, P. S. (1995). Vicarious traumatization: An empirical study of the effects of trauma work on trauma therapists. Professional Psychology: Research and Practice, 26(6), 558-565. doi:10.1037/0735-7028.26.6.558

Pearlman, L. A. (1996). Psychometric review of TSI Belief Scale, revision L. In B. H. Stamm (Ed.), Measurement of stress, trauma, and adaptation (pp. 415-417). Lutherville, MD: Sidran Press.

Perfect, M. M., Turley, M. R., Carlson, J. S., Yohanna, J., \& Saint Gilles, M. P. (2016). Schoolrelated outcomes of traumatic event exposure and traumatic stress symptoms in students: A systematic review of research from 1990 to 2015. School Mental Health, 8, 7-43. doi:10.1007/s1231 0-016-9175-2

Perron, B., \& Hiltz, B. (2006). Burnout and secondary trauma among forensic interviewers of abused children. Child \& Adolescent Social Work Journal, 23(2), 216-234. doi:10.1007/s10560-005-0044-3

Pierce, H., \& Lilly, M. M. (2012). Duty-related trauma exposure in 911 telecommunicators: 
Considering the risk for posttraumatic stress. Journal of Traumatic Stress, 25(2), 211215. doi:10.1002/jts. 21687

Pines, A., Aronson, E., \& Kafry, D. (1981). Combatting staff burnout in child care centers: A case study. Child Care Quarterly, 9, 5-16. doi:10.1007/bf01555032

Pines, A. M., \& Aronson, E. (1988). Career burnout: Causes and cures. New York: Free Press.

Podolsky, A., Kini, T., Bishop, J., \& Darling-Hammond, L. (2016). Solving the teacher shortage: How to attract and retain excellent educators. Retrieved from https://www.learningpolicyinstitute.org/sites/default/files/productfiles/Solving_Teacher_Shortage_Attract_Retain_Educators_REPORT.pdf

Polit, D. F., \& Beck, C. T. (2010). Generalization in quantitative and qualitative research: Myths and strategies. International Journal of Nursing Studies, 47(11), 1451-1458. doi:10.1016/j.ijnurstu.2010.06.004

Price, T. (2017, April 19). Secretary Price announces HHS strategy for fighting opioid crisis. U.S. Department of Health \& Human Services. Retrieved from https://www.hhs.gov/about/leadership/secretary/speeches/2017-speeches/secretary-priceannounces-hhs-strategy-for-fighting-opioid-crisis/index.html

Qualtrics. (2014). ESOMAR 28: 28 Questions to help buyers of online samples. Retrieved from https://www.esomar.org/what-we-do/code-guidelines/28-questions-to-help-buyers-ofonline-samples

Quast, T. (2018) State-level variation in the relationship between child removals and opioid prescriptions. Child Abuse \& Neglect, 86, 306-313. doi:10.1016/j.chiabu.2018.10.001 Radel, L., Baldwin, M., Crouse, G., Ghertner, R., \& Waters, A. (2018). Substance use, the 
opioid epidemic, and the child welfare system: Key findings from a mixed methods study. ASPE Research Brief. Office of the Assistant Secretary for Planning and Evaluation. U. S. Department of Health and Human Services. Retrieved from https://aspe.hhs.gov/system/files/pdf/258836/SubstanceUseChildWelfareOverview.pdf

Radel, L., Baldwin, M., Crouse, G., Ghertner, R., \& Waters, A. (2018). Medication-assisted treatment for opioid use disorder in the child welfare context: Challenges and opportunities. Retrieved from U.S. Department of Health and Services, Office of the Assistant Secretary for Planning and Evaluation website: https://aspe.hhs.gov/system/files/pdf/260121/MATChildWelfare.pdf

Rader, J. (2018, November). In the opioid crisis, here's what it takes to save a life. [Video file]. Retrieved from https://www.ted.com/talks/jan_rader_in_the_opioid_crisis_here_s_what_it_takes_to_save a_life?language $=$ en

Rauvola, R. S., Vega, D. M., \& Lavigne, K. N. (2019). Compassion fatigue, secondary traumatic stress, and vicarious traumatization: A qualitative review and research agenda. Occupational Health Science, 3(3), 297-336. doi:10.1007/s41542-019-00045-1

Reilly, K. (2018, September 13). 'I work 3 jobs and donate blood plasma to pay the bills.' This is what it's like to be a teacher in America. Time Magazine. Retrieved from https://time.com/5395001/teacher-in-america/

Reisfield, G. M., Bertholf, R. L., \& Wilson G. R. (2007). Opiates and opioids: What a difference a name makes. Journal of Opioid Management, 3(4), 179-80. Retrieved from https://www.wmpllc.org/ojs/index.php/jom/article/viewFile/1013/1023 
Ridgard, T. J., Laracy, S. D., Dupaul, G. J., Shapiro, E. S., \& Power, T. J. (2015). Traumainformed care in schools: A social justice imperative. Communique, 44(2), pp. 1, 12, 1415. Retrieved from https://www.nasponline.org/publications/periodicals/communique/issues/volume-44issue-2/trauma-informed-care-in-schools-a-social-justice-imperative

Ringer, J. L. (2017). An analysis of stress and self-efficacy experienced by general and special educators (Doctoral dissertation). University of Nevada, Las Vegas). Retrieved from https://digitalscholarship.unlv.edu/thesesdissertations/3028

Robinson, B. (2005). Exploring career satisfaction, burnout, and compassion fatigue as indicators of the quality of career engagement of public school educators. Dissertation Abstracts International: Section B. Sciences and Engineering, 66(12-B), AAI3201740.

Rojas-Flores, L., Herrera, S., Currier, J. M., Foster, J. D., Putman, K. M., Roland, A., \& Foy, D. W. (2015). Exposure to violence, posttraumatic stress, and burnout among teachers in El Salvador: Testing a mediational model. International Perspectives in Psychology: Research, Practice, Consultation, 4, 98-110. doi:10.1037/ipp0000029

Ross, S. W., Romer, N., \& Horner, R. H. (2012). Teacher well-being and the implementation of school-wide positive behavior interventions and supports. Journal of Positive Behavior Interventions, 14(2), 118-128. doi:10.1177/1098300711413820

Rudgley, R. (2000). The lost civilization of the Stone Age. New York, NY: Simon \& Schuster.

Ruel, E., Wagner, W., \& Gillespie, B. (2016). The practice of survey research: Theory and applications. Los Angeles, CA: Sage.

Rzeszutek, M., Partyka, M., \& Gołąb, A. (2015). Temperament traits, social support, and secondary traumatic stress disorder symptoms in a sample of trauma therapists. 
Professional Psychology: Research and Practice, 46(4), 213-220.

doi:10.1037/pro0000024

Salkind, N. (2017). Statistics for people who think they hate statistics. Los Angeles, CA: Sage.

Salston, M., \& Figley, C. R. (2003). Secondary traumatic stress effects of working with survivors of criminal victimization. Journal of Traumatic Stress, 16(2), 167-174.

doi:10.1023/a:1022899207206

Samuelowicz, K., \& Bain, J. D. (2001). Revisiting academics' beliefs about teaching and learning. Higher Education, 41, 299-325. doi:10.1023/A:1004130031247

Sareen, J. (2014). Posttraumatic stress disorder in adults: Impact, comorbidity, risk factors, and treatment. The Canadian Journal of Psychiatry, 59(9), 460-467. doi:10.1177/070674371405900902

Saunders, B. E., \& Adams, Z. W. (2014). Epidemiology of traumatic experiences in childhood. Child and Adolescent Psychiatric Clinics of North America, 23(2), 167-184. doi:10.1016/j.chc.2013.12.003

Sauter, S. (2017). Forward 1 in T. McIntyre, S. McIntyre, \& D. Francis (Eds.), Educator stress: An occupational health perspective (pp. vii-viii). Cham, Switzerland: Springer International Publishing.

Schepers, O. (2017). A teacher at risk: Giving voice to teacher secondary traumatic stress. (Doctoral dissertation). University of Colorado, Boulder, CO. Retrieved from https://scholar.colorado.edu/concern/graduate_thesis_or_dissertations/3x816m761

Schindler, A. (2019). Attachment and substance use disorders-Theoretical models. Frontiers in psychiatry, 10, 727. doi:10.3389/fpsyt.2019.00727 
Schorchit, N. (2017, March 1). As opioid crisis alarms communities, drug education now starts in kindergarten. NEAToday. Retrieved from http://neatoday.org/2017/03/01/opioid-crisis$\underline{\text { schools/ }}$

Setti, I., \& Argentero, P. (2012). Secondary Traumatic Stress Scale--Italian Version [Database record]. PsycTESTS. doi: http://dx.doi.org/10.1037/t21882-000

Sikes, A., Walley, C., \& Hays, D. G. (2012). A qualitative examination of ethical and legal considerations regarding dating violence. Journal of Interpersonal Violence, 27, 1474 1488. doi: $10.1177 / 0886260511425791$

Simon, M. K., \& Goes, J. (2013). Dissertation and scholarly research: Recipes for success. Seattle, WA: Dissertation Success, LLC. Retrieved from http://www.dissertationrecipes.com/

Slavich, G. M., \& Shields, G. S. (2018). Assessing lifetime stress exposure using the Stress and Adversity Inventory for Adults (Adult STRAIN): An overview and initial validation. Psychosomatic Medicine, 80(1), 17-27. doi:10.1097/psy.0000000000000534

Slesnick, N., Feng, X., Brakenhoff, B., \& Brigham, G. S. (2014) Parenting under the influence: The effects of opioids, alcohol and cocaine on mother-child interaction. Addictive Behaviors, 39(5), 897-900. doi:10.1016/j.addbeh.2014.02.003

Smith Hatcher, S., Bride, B. E., Oh, H., Moultrie King, D., \& Franklin Catrett, J. (2011). An assessment of secondary traumatic stress in juvenile justice education workers. Journal of Correctional Health Care, 17(3), 208-217. doi:10.1177/1078345811401509

Smith, V. C., \& Wilson, C. R., (2016). Families affected by parental substance use. Pediatrics, 138(2), 1-13. doi:10.1542/peds.2016-1575 
Spielberger, C. D., Gorsuch, R. L., Lushene, R., Vagg, P. R., \& Jacobs, G. A. (1983). Manual for the State-Trait Anxiety Inventory. Palo Alto, CA: Consulting Psychologists Press.

Sprang, G., Ford, J., Kerig, P., \& Bride, B. (2019). Defining secondary traumatic stress and developing targeted assessments and interventions: Lessons learned from research and leading experts. Traumatology, 25(2), 72-81. doi:10.1037/trm0000180

Stamm, B. H. (1996). Measurement of stress, trauma, and adaptation. Lutherville, MD: Sidram Press.

Stamm, B. H. (1999). Introduction to the first edition. In B. H. Stamm (Ed.), Secondary traumatic stress: Self-care issues for clinicians, researchers, \& educators (2nd ed., pp. xxxii-xliii). Lutherville, MD: Sidran Press.

Stamm, B. H. (2002). Measuring compassion satisfaction as well as fatigue: Developmental history of the Compassion Satisfaction and Fatigue Test. In C. R. Figley (Ed.), Psychosocial stress series, no. 24. Treating compassion fatigue (p. 107-119). New York, NY: Brunner-Routledge.

Stamm, B. H. (2005). The ProQOL manual: The Professional Quality of Life Scale: Compassion Satisfaction, Burnout \& Compassion Fatigue/Secondary Trauma Scales. Retrieved from http://www.compassionfatigue.org/pages/ProQOLManualOct05.pdf

Stamm, B. H. (2009). The concise ProQOL manual. Pocatello, ID: ProQOL.org.

Stamm, B. H. (2010). The concise ProQOL manual (2nd ed.). Pocatello, ID: ProQOL.org.

STAT: Support for Teachers Affected by Trauma. (2020). Retrieved from https://statprogram.org/

Steel, J. L., Dunlavy, A. C., Stillman, J., \& Pape, H. C. (2011). Measuring depression and PTSD 
after trauma: Common scales and checklists. Injury, 42(3), 288-300.

doi:10.1016/j.injury.2010.11.045

Stevens, J. (2019, May 21). Bad news-good news: Each additional ACE increases opioid rate by 17\%; each ACE-informed treatment visit reduces it by $2 \%$ [Web log post]. Retrieved from https://www.acesconnection.com/blog/bad-news-good-news-each-additional-aceincreases-opioid-relapse-rate-by-17-each-ace-informed-treatment-visit-reduces-it-by-2

Strathearn, L., Mertens, C. E., Mayes, L., Rutherford, H., Rajhans, P., Xu, G., .. Kim, S. (2019). Pathways relating the neurobiology of attachment to drug addiction. Frontiers in Psychiatry, 10, 1-15. doi:10.3389/fpsyt.2019.00737

Strauss, J. N. (2013). Autism as culture. In L. Davis (Ed.), The disability studies reader (pp. 460484). New York, NY: Routledge.

Streeck-Fischer, A., \& Van der Kolk, B. A. (2000). Down will come baby, cradle and all: Diagnostic and therapeutic implications of chronic trauma on child development. Australian \& New Zealand Journal of Psychiatry, 34(6), 903-918. doi:10.1080/000486700265

Stulac, S., Bair-Merritt, M., Wachman, E. M., Augustyn, M., Howard, C., Madoor, N., \& Costello, E. (2019). Children and families of the opioid epidemic: Under the radar. Current Problems in Pediatric and Adolescent Health Care, 49(8), 100637. doi:10.1016/j.cppeds.2019.07.002

Substance Abuse and Mental Health Services, Center for Behavioral Health Statistics and Quality. (2016). DSM-5 changes: Implications for child serious emotional disturbance. Retrieved from https://www.ncbi.nlm.nih.gov/books/NBK519708/

Sugai, G., \& Horner, R. (2002). The evolution of discipline practices: School-wide positive 
behavior supports. Child \& Family Behavior Therapy, 24(1-2), 23-50.

doi:10.1300/j019v24n01_03

Sutcher, L., Darling-Hammond, L., \& Carver-Thomas, D. (2019). Understanding teacher shortages: An analysis of teacher supply and demand in the United States. Education Policy Analysis Archives, 27, 35. doi:10.14507/epaa.27.3696

Swartz, M. K. (2018). Opioids: A pediatric epidemic. Journal of Pediatric Health Care, 32(2), 115-116. doi:10.1016/j.pedhc.2018.01.002

Taber, K. S. (2017). The use of Cronbach's alpha when developing and reporting research instruments in science education. Research in Science Education, 48(6), 1273-1296. doi:10.1007/s11165-016-9602-2

Ting, L., Jacobson, J., Sanders, S., Bride, B. E., \& Harrington, D. (2005). The Secondary Traumatic Stress Scale: Confirmatory factor analyses in a national sample of mental health social workers. Journal of Human Behavior in the Social Environment, 11(3-4), 177-194 doi:10.1300/j137v11n03_09

Tosone, C., McTighe, J. P., Bauwens, J., \& Naturale, A. (2011). Shared traumatic stress and the long-term impact of 9/11 on Manhattan clinicians. Journal of Traumatic Stress, 24(5), 546-552. doi:10.1002/jts.20686

Townsend, S. M., \& Campbell, R. (2009). Organizational correlates of secondary traumatic stress and burnout among sexual assault nurse examiners. Journal of Forensic Nursing, 5(2), 97-106. doi:10.1111/j.1939-3938.2009.01040.x

Travers, C. (2017). Current knowledge in the nature, prevalence, sources and potential impact of teacher stress. In T. McIntyre, S. McIntyre, \& D. Francis (Eds.), Educator stress: An 
occupational health perspective (pp. 23-54). Cham, Switzerland: Springer International Publishing.

Trigwell, K. (2011). Relations between teachers' emotions in teaching and their approaches to teaching in higher education. Instructional Science, 40(3), 607-621. doi:10.1007/s11251011-9192-3

Turliuc, M. N., Măirean, C., \& Turliuc, M. D. (2015). Secondary Traumatic Stress ScaleRomanian Version. PsycTESTS. doi:10.1037/t44219-000

Urdan, T. (2017). Statistics in plain English (4th ed.). New York, NY: Routledge.

U. S. Department of Education. (2018). Combating the opioid crisis: Schools, students and families. Retrieved from https://www2.ed.gov/documents/opioids/site.pdf

U.S. Department of Education, National Center for Education Statistics Schools and Staffing Survey (SASS). (2011). Table 2. Average and median age of public school teachers and percentage distribution of teachers, by age category, sex, and state: 2011-12 [Data file]. Retrieved from https://nces.ed.gov/surveys/sass/tables/sass1112_2013314_t1s_002.asp

U.S. Department of Health and Human Services, Administration for Children and Families. (n.d.). Trauma: What is historical trauma? Retrieved from https://www.acf.hhs.gov/trauma-toolkit/trauma-concept

U.S. Department of Health and Human Services, Administration for Children and Families, Administration on Children, Youth and Families, Children's Bureau. (2013). Child maltreatment 2012. Retrieved from https://www.acf.hhs.gov/sites/default/files/cb/cm2012.pdf

U.S. Department of Health \& Human Services, Administration for Children and Families, Administration on Children, Youth and Families, Children's Bureau. (2018). Child 
maltreatment 2016. Retrieved from

https://www.acf.hhs.gov/sites/default/files/cb/cm2016.pdf

U.S. Department of Labor, Bureau of Labor Statistics. (2015). News release. Employer-reported workplace injuries and illnesses - 2014. Retrieved from https://www.bls.gov/news.release/archives/osh_10292015.pdf

VanBergeijk, E. O., \& Sarmiento, T. L. (2006). The consequences of reporting child maltreatment: Are school personnel at risk for secondary traumatic stress? Brief Treatment and Crisis Intervention, 6(1), 79-98. doi:10.1093/brief-treatment/mhj003

Van der Kolk, B. A. (2005). Developmental trauma disorder: Toward a rational diagnosis for children with complex trauma histories. Psychiatric Annals, 35(5), 401-408. doi:10.3928/00485713-20050501-06

Wang, L., Zhang, L., Armour, C., Cao, C., Qing, Y., Zhang, J., ... Fan, G. (2015). Assessing the underlying dimensionality of DSM-5 PTSD symptoms in Chinese adolescents surviving the 2008 Wenchuan earthquake. Journal of Anxiety Disorders, 31, 90-97. doi:10.1016/j.janxdis.2015.02.006

Watson, D. (2005). Rethinking the mood and anxiety disorders: A quantitative hierarchical model for DSM-V. Journal of Abnormal Psychology, 114(4), 522-536. doi:10.1037/0021-843x.114.4.522

Watson, D. (2009). Differentiating the mood and anxiety disorders: A quadripartite model. Annual Review of Clinical Psychology, 5(1), 221-247. doi:10.1146/annurev.clinpsy.032408.153510

Wee, D. F., \& Myers, D. (2002). Stress response of mental health workers following disaster: 
The Oklahoma City bombing. In E. Figley (Ed.), Treating compassion fatigue (pp. 5784). New York: Bruner/Mazel.

Weigold, A., Weigold, I. K., \& Russell, E. J. (2013). Examination of the equivalence of selfreport survey-based paper-and-pencil and internet data collection methods. Psychological Methods, 18(1), 53-70. doi:10.1037/a0031607

Weiss, A. J., Elixhauser, A., Barrett, M. L. Steiner, C. A., Bailey, M. K., \& O’Malley, L. (2017). Opioid-related inpatient stays and emergency department visits by state, 2009-2014 (Statistical Brief No. 219). Retrieved from Agency of Healthcare and Research Quality website: https://www.hcup-us.ahrq.gov/reports/statbriefs/sb219-Opioid-Hospital-StaysED-Visits-by-State.pdf

Welby, K. (2019). The implications of the opioid epidemic on select elementary schools in crisis regions of the Northeast: A multiple case study investigation (Doctoral dissertation). Southern New Hampshire University). Manchester, NH. Retrieved from https://academicarchive.snhu.edu/handle/10474/3569

Welsh, J., Knight, M., Hou, S., Malowney, M., Schram, P., Sherritt, L, \& Boyd, W. (2017). Association between substance use diagnoses and psychiatric disorders in an adolescent and young adult clinic-based population. Journal of Adolescent Health, 60(6), 648-652. doi:10.1016/j.jadohealth.2016.12.018

Welsh J. W., Tretyak V., \& Rappaport N. (2018). The opioid crisis and schools-A commentary. Journal of School Health, 88(5), 337-340. doi:10.1111/josh.1267

Williams, M. T., Metzger, I. W., Leins, C., \& DeLapp, C. (2018). Assessing racial trauma within a DSM-5 framework: The UConn Racial/Ethnic Stress \& Trauma Survey. Practice Innovations, 3(4), 242-260. doi:10.1037/pri0000076 
Wolf-Prusan, L. (2014). The impact of student gang/gun-related homicide on urban high school teachers (Doctoral dissertation). University of California, Los Angeles, CA. Retrieved from https://cloudfront.escholarship.org/dist/prd/content/qt2sk4z9sv/qt2sk4z9sv.pdf

Woodbridge, M. W., Sumi, W. C., Thornton, S. P., Fabrikant, N., Rouspil, K. M., Langley, A. K., \& Kataoka, S. H. (2016). Screening for trauma in early adolescence: Findings from a diverse school district. School Mental Health, 8, 89-105. doi:10.1007/s12310-015-91695

Yager, T. J., Gerszberg, N., \& Dohrenwend, B. P. (2016). Secondary traumatization in Vietnam veterans' families. Journal of Traumatic Stress, 29(4), 349-355. doi:10.1002/jts.22115

Yazzie-Mintz, E. (2007). Voices of students on engagement: A report on the 2006 High School Survey of Student Engagement. Bloomington, IN: Center for Evaluation and Education Policy, Indiana University.

Yildirim, G., Kidak, L. B., \& Yurdabakan, I. (2018). Secondary Traumatic Stress Scale: An adaptation study/Ikincil Travmatik Stres Olcegi: Bir uyarlama calismasi. Anatolian Journal of Psychiatry/Anadolu Psikiyatri Dergisi, 19(1), 45-51. doi:10.5455/apd.247563

Young, A. (1995). The harmony of illusions: Inventing posttraumatic stress disorder. Princeton, NJ: Princeton University Press.

Young, A. (2004). When traumatic memory was a problem: On the historical antecedents of PTSD. In G. R. Rosen (Ed.), Posttraumatic stress disorder: Issues and controversies (pp. 127-146). Chichester, UK: Wiley.

Zimmet, P. Z. (2017). Diabetes and its drivers: the largest epidemic in human history? Clinical Diabetes and Endocrinology, 3(1). doi:10.1186/s40842-016-0039-3 


\title{
Appendix A. Questions on the Secondary Traumatic Stress Scale (STSS; Bride et al., 2004)
}

\author{
APPENDIX \\ SECONDARY TRAUMATIC STRESS SCALE
}

The following is a list of statements made by persons who have been impacted by their work with traumatized clients. Read each statement, then indicate how frequently the statement was true for you in the past seven (7) days by circling the corresponding number next to the statement.

\begin{tabular}{|c|c|c|c|c|c|}
\hline & Never & Rarely & Occasionally & Often & Very Often \\
\hline 2. My heart started pounding when I thought about my work with clients. & 1 & 2 & 3 & 4 & 5 \\
\hline 4. I had trouble sleeping. & 1 & 2 & 3 & 4 & 5 \\
\hline 5. I felt discouraged about the future. & 1 & 2 & 3 & 4 & 5 \\
\hline 6. Reminders of my work with clients upset me. & 1 & 2 & 3 & 4 & 5 \\
\hline 9. I was less active than usual. & 1 & 2 & 3 & 4 & 5 \\
\hline 10. I thought about my work with clients when I didn't intend to. & 1 & 2 & 3 & 4 & 5 \\
\hline 11. I had trouble concentrating. & 1 & 2 & 3 & 4 & 5 \\
\hline 12. I avoided people, places, or things that reminded me of my work with clients. & 1 & 2 & 3 & 4 & 5 \\
\hline 13. I had disturbing dreams about my work with clients. & 1 & 2 & 3 & 4 & 5 \\
\hline 14. I wanted to avoid working with some clients. & 1 & 2 & 3 & 4 & 5 \\
\hline
\end{tabular}

Copyright 1999, Brian E. Bride.

NOTE: "Client" is used to indicate persons with whom you have been engaged in a helping relationship. You may substitute another noun that better represents your work such as consumer, patient, recipient, and so forth. 


\title{
Appendix B. Questionnaire Developed and Distributed through Qualtrics
}

\author{
Secondary Traumatic Stress of Teachers \\ in School Communities Impacted by the Opioid \\ Epidemic
}

Introduction

The purpose of this survey is to explore the experience of teachers who work with children who have experienced trauma. People who support others, like nurses in an emergency room or social workers after a natural disaster, can sometimes be affected by a type of stress called secondary traumatic stress. Secondary traumatic stress is defined as behavior and emotions that result from supporting others who have experienced trauma. This survey will help gather information about teacher, focusing on secondary traumatic stress.

Your participation in this research is voluntary. You may choose not to participate. If you decide to participate in this research survey, you may withdraw at any time. If you decide not to participate in this study or if you withdraw from participating at any time, you will not be penalized.

This procedure involves completing the online survey which will take approximately 2030 minutes. Your responses will be anonymous and confidential. At no time is identifying information collected for the survey, like your name, email address, or IP address. To read your full rights and a more complete explanation of protections to your confidentiality, please click below:

\section{Full Consent Form}

A. Clicking on the "Agree" button indicates the following:

- You have read the introductory consent information and you know that you have the option of reading your complete rights by downloading the full consent form (above);

- You voluntarily agree to participate in the study;

- You are at least 18 years old. 
Agree

Disagree

B. To read more about how Qualtrics protects your data, click here:

\section{Qualtrics Data \\ Protection}

C. Which of the following best describes your profession?

- Firefighter

- Teacher

- Lawyer

- Doctor

- None of the above

\section{Survey Begins}

1. What is your age?
20-30 years old
31-40 years old
41-50 years old
51-60 years old
Over 60 years old
Prefer not to answer

2. What is your preferred gender designation?

Female

Male

Non-binary

Prefer not to answer

Other

3. With which racial/ethnic identity do you identify?

American Indian or Alaskan Native

Asian 
Black or African American

Hispanic/Latino

Native Hawaiian or other Pacific Islander

White

Combination of two or more races

Prefer not to identify

Other:

4. What is your marital status?

Single

In a committed partnership

Married

Divorced

Widowed

Prefer not to identify

Other:

5. In the last year, your primary teaching assignment was in which of the following $\operatorname{grade}(\mathrm{s})$ ?

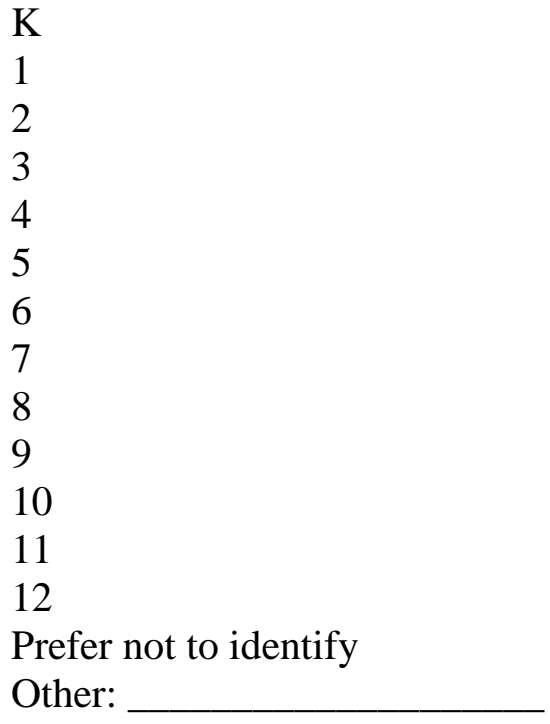

6. In the last year, your primary teaching assignment was in which of the following setting(s)?

Elementary (as identified by your state)

Middle school (as identified by your state)

High school (as identified by your state)

Special education (setting outside of the general education classroom setting, (as identified by your state)

Blended (teach a variety of classes/grades from K-8 or K-12) 
state)

Alternative education (continuation, credit recover, etc., as identified by your

Prefer not to identify

Other:

7. Number of years teaching in all settings:

8. Zip code of current school location:

9. Which of the following, if any reflect the types of trauma experienced by your students? Please select all that apply. More information about the type of trauma is provided here:

Definition of Trauma

\author{
EMOTIONAL ABUSE \\ PHYSICAL ABUSE \\ SEXUAL ABUSE \\ EMOTIONAL NEGLECT \\ PHYSICAL NEGLECT \\ DOMESTIC VIOLENCE \\ HOUSEHOLD SUBSTANCE ABUSE \\ HOUSEHOLD MENTAL ILLNESS \\ INCARCERATED HOUSEHOLD MEMBER \\ WITNESS VIOLENCE \\ FELT RACIAL/ETHNIC DISCRIMINATION \\ ADVERSE NEIGHBORHOOD EXPERIENCE \\ LIVED IN FOSTER CARE \\ EXPERIENCED DEATH IN THE FAMILY \\ NONE
}

10. On average, what percentage of students in your class(es) have experienced one or more of the above?

Percentage:

I do not know

\title{
STSS Survey Questions
}

Secondary traumatic stress is defined as behavior and emotions that result from supporting others who have experienced trauma. The following is a list of statements made by persons who have been impacted by their work with traumatized clients or students. Read each 
statement, then indicate how frequently the statement was true for you in the past seven (7) days by circling the corresponding number next to the statement.

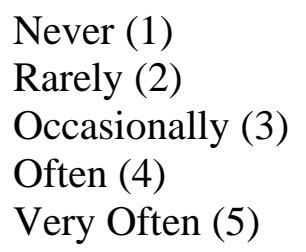

1. I felt emotionally numb.

2. My heart started pounding when I thought about my work with the students.

3. It seemed as if I was reliving the trauma(s) experienced by my student(s).

4. I had trouble sleeping.

5. I felt discouraged about the future.

6. Reminders of my work with students upset me.

7. I had little interest in being around others.

8. I felt jumpy.

9. I was less active than usual.

10. I thought about my work with students when I didn't intend to.

11. I had trouble concentrating.

12. I avoided people, places, or things that reminded me of my work with students.

13. I had disturbing dreams about my work with students.

14. I wanted to avoid working with some students.

15. I was easily annoyed.

16. I expected something bad to happen.

17. I noticed gaps in my memory about parts of my teaching day.

\section{Personal Response Questions (Optional)}

The following questions are optional. Please provide detailed insight, if you are comfortable.

a. What do you find difficult about supporting students who experience significantly adversity and trauma? How is it difficult?

b. What do you find positive or rewarding about supporting students who experience significant adversity and trauma? How is it positive for you? 
d. What else would you like to say about your experiences supporting children who experience significant adversity and trauma?

\section{POSSIBLE FUTURE INTERVIEW}

Although not a part of this survey, if you would be interested in being interviewed about the topic of secondary stress in teaching, you can let the researcher know about your interest by sending an email to this email address: 4sts.interview@gmail.com.

Secondary stress in teaching has not been studied extensively on a national level. This could be groundbreaking research that values voices from actual teachers, as opposed to generalizations from other helping professions.

If you are interested in being contacted, you will receive information about possible future interviews in a one-time only email. As with this survey, you will be under no obligation to participate. The email is simply to receive information about the possibility of a future interview.

\section{SUPPORT MATERIAL FOR SECONDARY STRESS}

Thank you for your participation in this survey. If you would like to access support materials and resources for teachers who are experiencing stress and/or secondary stress, please click here:

\section{Support}




\section{Appendix C. Permission to Use the Secondary Traumatic Stress Scale (Bride)}

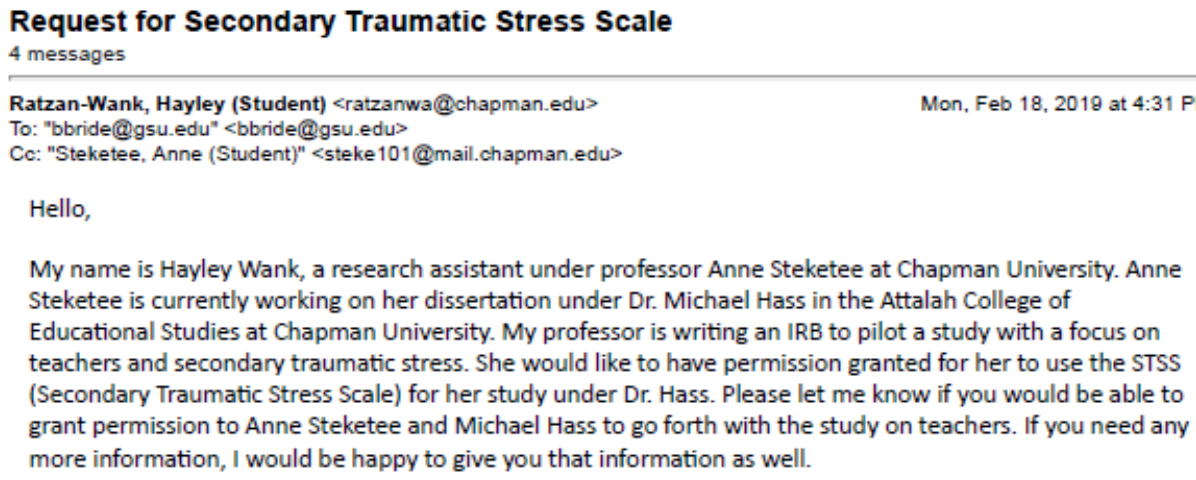

Best,

Hayley Wank 
From: Brian Bride <bbride@gsu.edu>

Sent: Tuesday, February 19, 2019 8:23 AM

To: Ratzan-Wank, Hayley (Student)

Cc: Steketee, Anne (Student)

Subject: Re: Request for Secondary Traumatic Stress Scale

[Quoted text hidden]

Anne Steketee <steke101@mail.chapman.edu>

Tue, Feb 19, 2019 at 11:58 AM

To: "Ratzan-Wank, Hayley (Student)" <ratzanwa@chapman.edu>

Hayley, this is amazing!!!!

a

[Quoted text hidden] 


\section{Appendix D. Keys to Numerical Codes}

\begin{tabular}{|c|c|c|c|c|c|c|c|}
\hline 4 & A & B & c & D & E & $\mathrm{F}$ & G \\
\hline 1 & Code No. & $\begin{array}{l}\text { Opioid Epidemic Zone (1=low; } \\
\text { 2=med; 3=high) }\end{array}$ & Gender by No. & Racial/ Ethnicity by No. & Marital Status & $\begin{array}{l}\text { Primary Teaching Assignment: Setting by } \\
\text { No. }\end{array}$ & Age \\
\hline 2 & & 1 Avoid & Female & American Indian or Alaskan Native & Divorced & $\begin{array}{l}\text { Alternative education (continuation, credit } \\
\text { recovery, etc., as identified by your state) }\end{array}$ & $20-30 \mathrm{yr}$ \\
\hline & & 2 Intrus & & & In a committed & $\begin{array}{l}\text { Blended (teach a variety of classes/grades } \\
\text { from K-8 or K-12) }\end{array}$ & \\
\hline 4 & & 3 Arous & Non-binar & Black or African American & Married & Elementary (as identified by your state) & $41-50 \mathrm{yr}$ \\
\hline 5 & & 4 & Other & Combination of two or more races & Other & High School (as identified by your state) & $51-60 \mathrm{yr}$ \\
\hline 6 & & 5 & & Hispanic/Latino & Single & Middle School (as identified by your state) & over $60 \mathrm{yr}$ \\
\hline 7 & & 6 & & Native Hawaiian or other Pacific Islander & Widowed & Multiple & $\begin{array}{l}\text { Prefer } \\
\text { not to } \\
\text { answer }\end{array}$ \\
\hline 8 & & 7 & & Other & & Special Education & \\
\hline 9 & & 8 & & Prefer not to identify & & Other & \\
\hline 10 & & 9 & & White & & Prefer not to identify & \\
\hline 11 & & 10 & & & & & \\
\hline
\end{tabular}




\section{Appendix E. Permission to use Akoglu Figure}

$5 / 25 / 2020$

CHAPMAN

UNIVERSITY
Chapman Universty Mal - Re: Fwd: From research in Southem Callfornla

Anne Steketee <steke101@mail.chapman.edu

\section{Re: Fwd: From research in Southern California}

Haldun Akoğlu <drhaldun@gmail.com>

Mon, May 25, 2020 at 11:54 AM

To: Anne Steketee <steke101@mail.chapman.edu>

Dear Ms Steketee,

I officially give you my and publisher's permission to reproduce the table by citing the original article.

Thanks for your interest and hope you a great success while you are defending your thesis.

Kindest regards

Haldun AKOGLU, MD

Professor of Emergency Medicine

Marmara University School of Medicine, Istanbul, Turkey @marmaraacil

Emergency Medicine Association of Turkey (EMAT). Executive Board Member

Editor-in-Chief, Turkish Journal of Emergency Medicine @TurkJEmergMed

Editor and Founder, Acilci.Net \#FOAMed @acilci_net

COCHRANE Prehospital and Emergency Care Field, PEARL Author

IFEM International Federation of Emergency Medicine, Task Force Member

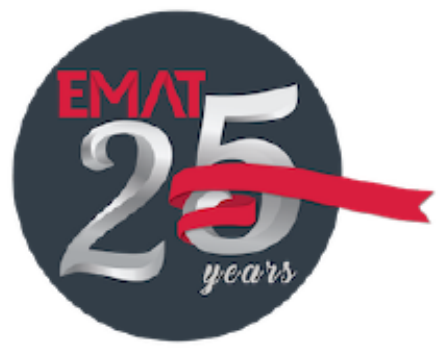

On 23 May 2020 08:18 +0300, Anne Steketee <steke101@mail.chapman.edu>, wrote:

From: Anne Steketee <steke101@mail.chapman.edu>

Date: Fri, May 22, 2020 at 10:18 PM

Subject: From research in Southern California

To: <haldun.akoglu@marmara.edu>

Hi, Dr. Akoglu-

I am finishing my dissertation at Chapman University and wondered if I could have your permission to use this figure

from your article. User's guide to correlation coefficients. I would credit you and your article. It is one of the best charts I have found in the literature.

Thank you for considering this request. I hope you are staying well in this time of COVID. 


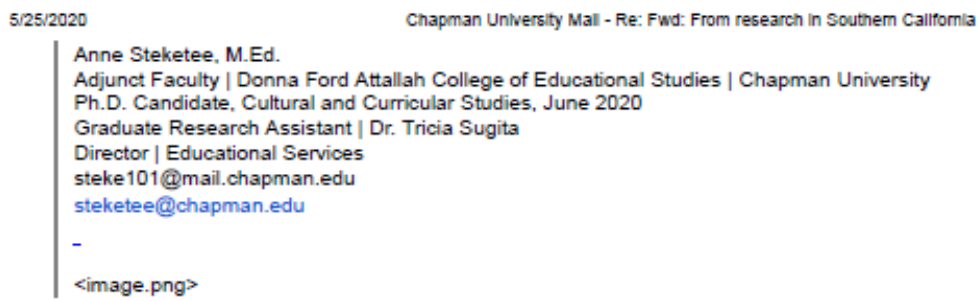

